Sapali Kirst

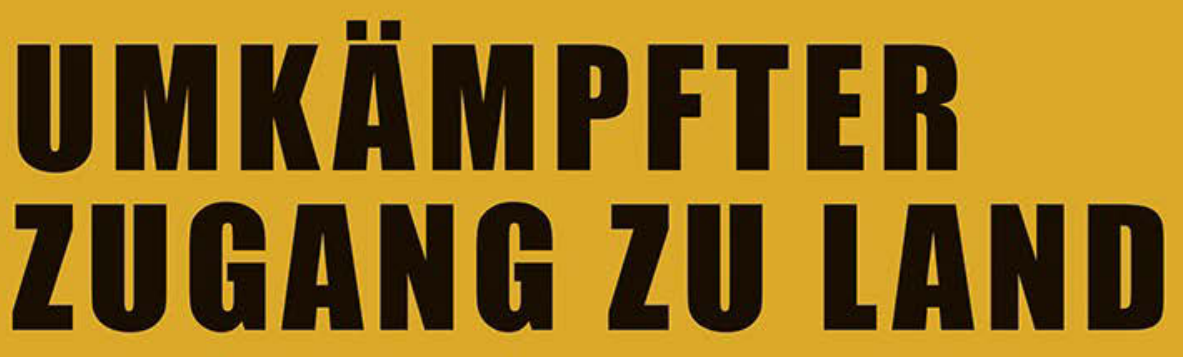

Land IPahbing, Konfilikto und die Rolle traditioneller Autoritäten in Ghana

[transcript] G/obalstudies 
Sarah Kirst

Umkämpfter Zugang zu Land

Global Studies 
Sarah Kirst hat am Otto-Suhr-Institut für Politikwissenschaft der Freien Universität Berlin promoviert. Mit einem regionalen Fokus auf Subsahara-Afrika forscht sie zu Konflikten um Land und Ressourcen, Land und sozialer Zugehörigkeit, traditioneller Herrschaft, Gender- und Ungleichheitsverhältnissen. 
Sarah Kirst

\section{Umkämpfter Zugang zu Land}

Land grabbing, Konflikte und die Rolle traditioneller Autoritäten in Ghana 
Die Publikation wurde durch eine Ko-Finanzierung für Open-Access-Monografien und -Sammelbände der Freien Universität Berlin ermöglicht.

\section{Bibliografische Information der Deutschen Nationalbibliothek}

Die Deutsche Nationalbibliothek verzeichnet diese Publikation in der Deutschen Nationalbibliografie; detaillierte bibliografische Daten sind im Internet über http://dnb.d-nb.de abrufbar.

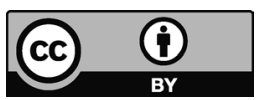

Dieses Werk ist lizenziert unter der Creative Commons Attribution 4.0 Lizenz (BY). Diese Lizenz erlaubt unter Voraussetzung der Namensnennung des Urhebers die Bearbeitung, Vervielfältigung und Verbreitung des Materials in jedem Format oder Medium für beliebige Zwecke, auch kommerziell. (Lizenztext: https://creativecommons.org/licenses/ by/4.0/deed.de)

Die Bedingungen der Creative-Commons-Lizenz gelten nur für Originalmaterial. Die Wiederverwendung von Material aus anderen Quellen (gekennzeichnet mit Quellenangabe) wie z.B. Schaubilder, Abbildungen, Fotos und Textauszüge erfordert ggf. weitere Nutzungsgenehmigungen durch den jeweiligen Rechteinhaber.

\section{Erschienen 2022 im transcript Verlag, Bielefeld (๑) Sarah Kirst}

Umschlaggestaltung: Maria Arndt, Bielefeld

Druck: Majuskel Medienproduktion $\mathrm{GmbH}$, Wetzlar

Print-ISBN 978-3-8376-5948-1

PDF-ISBN 978-3-8394-5948-5

https://doi.org/10.14361/9783839459485

Buchreihen-ISSN: 2702-9298

Buchreihen-eISSN: 2703-0504

Gedruckt auf alterungsbeständigem Papier mit chlorfrei gebleichtem Zellstoff. Besuchen Sie uns im Internet: https://www.transcript-verlag.de Unsere aktuelle Vorschau finden Sie unter www.transcript-verlag.de/vorschaudownload 


\section{Inhalt}

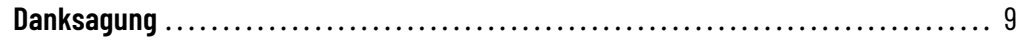

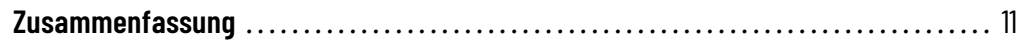

1 Einleitung ................................................... 13

1.1 Problemstellung ................................................ 17

1.2 Forschungskontext und Forschungsdesign ........................ 19

1.3 Fallauswahl................................................. 23

1.4 Struktur des Buches ........................................... 27

2 Land grabbing, Konflikte um Land und traditionelle Autoritäten - Stand der Forschung ............................................... 29

2.1 Land grabbing und Konflikte ................................... 30

2.2 Konflikte um Land und die Bedeutung sozialer Zugehörigkeit ............. 39

2.3 Traditionelle Autoritäten als Intermediäre ......................... 48

2.4 Bezug zur bestehenden Forschung - Anknüpfungspunkte und Leerstellen ..... 53

3 Zugang zu Land und Macht - Ein Analyserahmen für Konflikte im Kontext von land grabbing ........................................... 57

3.1 Zugang als analytische Kategorie in Konflikten um Land ................6 60

3.2 Macht als analytische Kategorie in Konflikten um Zugang zu Land........... 72

3.3 Zwischenfazit .............................................. 78

3.4 Operationalisierung der theoretischen Kategorien ................... 80

4 Forschungsdesign und methodisches Vorgehen ................... 93

4.1 Kollektive Fallstudie .......................................... 93

4.2 Kriterien der Fallauswahl ........................................ 94

4.3 Erhebung der Daten ..................................... 102 
4.4 Reflexion des methodischen Vorgehens ........................... 108

4.5 Auswertung und Analyse der Daten.............................. 111

5 Länderkontext - Traditionelle Landrechte und das chieftaincy-System in Ghana .............................. 117

5.1 Landrechte und die koloniale Erfindung von Traditionen ................... 117

5.2 Traditionelle Herrschaft und Landrechte im postkolonialen Ghana............. 119

5.3 Traditionelle Herrschaft in der Ashanti und Northern Region ............. 129

\section{Empirische Beispiele für Konflikte um Zugang}

zu Land im Kontext von land grabbing ............................ 135

6.1 Das ScanFarm-Projekt - Landvergabe, Konflikte, Konfliktakteure ............ 135

6.2 Das BioFuel Africa-Projekt - Landvergabe, Konflikte und Konfliktakteure ....... 147

7 Veränderung von Zugang zu Land im Kontext von land grabbing .......... 159

7.1 Kontrolle über Zugang zu Land vor der Landvergabe ................... 160

7.2 Zugang zu Land vor der Landvergabe an ScanFarm und BioFuel Africa ......... 162

7.3 Land grabbing und die Veränderung von Kontrolle und Zugang .............172

$7.4 \quad$ Zwischenfazit .............................................. 184

8 Die Bedeutung von Macht für Konflikte um Zugang zu Land ............. 187

8.1 Kontrolle des Zugangs zu Land über sichtbare, unsichtbare und versteckte Macht........................................ 189

8.2 Konflikte um Zugang zu Land im Spannungsfeld der drei Machtformen ........ 214

9 Fazit ..................................................... 219

9.1 Zusammenfassung und Diskussion der empirischen Erkenntnisse ........... 223

9.2 Formulierung von Hypothesen.................................... 240

9.3 Reichweite der Hypothesen ..................................... 245

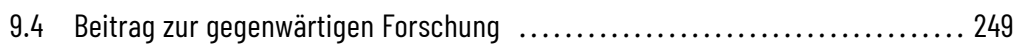

Literaturverzeichnis............................................ 255

Glossar ....................................................... 279

Abkürzungsverzeichnis.................................... 283

Abbildungsverzeichnis ........................................... 285 
Tabellenverzeichnis 



\section{Danksagung}

Dieses Buch ist eine kaum veränderte Version meiner Dissertation, die ich im November 2020 am Fachbereich Politik- und Sozialwissenschaft der Freien Universität Berlin eingereicht habe. Es ist ein Werk, zu dem viele Menschen über die letzten Jahre beigetragen haben, Menschen, ohne deren Unterstützung ich meine Dissertation weder begonnen noch abgeschlossen hätte und die entscheidend zu ihrer Entwicklung beigetragen haben. Ihnen allen möchte ich an dieser Stelle meinen tiefsten Dank aussprechen.

Ich danke meinen Interviewpartner*innen in Ghana für ihre Zeit und Geduld, ihre Offenheit und ihr Vertrauen. Außerdem danke ich Yawson Asamoah Kwame Mensa und Enock Asiedu Osarfo für viele Stunden ausdauernder Konsekutivübersetzung, die zuverlässige Unterstützung meiner Forschung, ihren kritischen Blick, ihre Gastfreundschaft und viele erhellende Gespräche zur ghanaischen Gesellschaft, Kultur und Sprache. Keep on shining!

Dieses Buch wäre nicht ohne GLOCON, die bereichernde gemeinsame Arbeit im Forschungsprojekt und die fantastische Betreuung entstanden. Ein riesiges Dankeschön gebührt meinen beiden Gutachterinnen Bettina Engels und Kristina Dietz. Ich hätte mir niemand Besseres für diese Aufgabe wünschen können. Vielen Dank für die ausdauernde Begleitung meiner Arbeit, die konstruktiv-kritischen Rückmeldungen, den klaren Durchblick in chaotischen Phasen sowie eure zugewandte, wertschätzende und motivierende Art. Merci beaucoup, muitíssimo obrigada und many thanks gehen außerdem an Louisa Prause, Jan Brunner und Mario Schenk: für stetigen Austausch, die geduldige Lektüre jedes Kapitels, akribische Kommentierungen und ihre Freundschaft.

Ebenso großer Dank gilt meiner Familie. Pedro, Léanne und Mia bin ich dankbar für ihre Zugeständnisse und ihre Geduld in all den Momenten, in denen ich mental oder physisch abwesend war und für ihre Nähe, die auch besteht, wenn ein Kontinent zwischen uns liegt. Catherine danke ich für ihre bedingungslose Unterstützung in allen Lebenslagen und ihr praktisches Wis- 
sen zur Stressbewältigung in besonders herausfordernden Phasen. Friedhelm bin ich zutiefst dankbar dafür, dass ich immer auf ihn zählen kann und Luzia für ihre Großzügigkeit sowie den schönsten Ort zum Schreiben.

Besondere Wertschätzung gilt überdies Barbara, Christoph und Lisa, die mir nicht nur eine temporäre Bleibe, sondern ein Berliner Zuhause gegeben und mich mit ihrer offenen, unkomplizierten Art bei sich willkommen geheiBen haben.

Für die Finanzierung der Forschung und die Möglichkeit, ein Projekt wie GLOCON realisieren zu können, danke ich dem Bundesministerium für Bildung und Forschung. 


\section{Zusammenfassung}

Im Spannungsfeld zwischen globaler Finanz-, Rohstoff- und Nahrungsmittelpreiskrise sowie dem Klimawandel und seinen möglichen Auswirkungen hat seit Mitte der $2000 e r$ Jahre die Nachfrage nach Land stark zugenommen. Ausdruck für diese Entwicklung ist der Anstieg großflächiger Aneignungen von Land durch nationale, internationale und multinationale Akteure. Ich bezeichne dieses Phänomen als land grabbing. Land grabbing lässt sich vor allem - wenn auch nicht ausschließlich - in Ländern des Globalen Südens beobachten. Besonders betroffen ist der afrikanische Kontinent. Dort eignen sich Investoren oftmals gewohnheitsrechtlich verwaltetes und kollektiv genutztes Land an, das den Großteil aller Nutzflächen ausmacht. Im Zusammenhang mit land grabbing treten in vielen Fällen Konflikte auf, die auf unterschiedlichen Ebenen und zwischen unterschiedlichen Akteuren verlaufen. Das verbindende Element in diesen Konflikten ist die Aushandlung von Zugang zu Land. Konflikte um Zugang verlaufen zwischen Akteuren, die Zugang zu Land kontrollieren, denjenigen die sich Zugang neuaneignen, und denjenigen, die Zugang über Dritte erlangen, um ihren Lebensunterhalt zu bestreiten. Je nach institutioneller Ausgestaltung des Zugangs zu Land sind in den Konflikten unterschiedliche Akteure involviert. In Subsahara-Afrika spielen traditionelle Autoritäten eine wichtige Rolle im Zusammenhang mit land grabbing. Sie sind die treuhänderischen Verwalter von gewohnheitsrechtlichem Land und in dieser Funktion auch Verhandlungspartner von Investoren in Bezug auf die großflächige Aneignung von Land. Das macht sie zu zentralen Akteuren in Konflikten, die in diesem Zusammenhang auftreten. Welche Rolle traditionelle Autoritäten in diesen Konflikten spielen, ist bislang jedoch kaum erforscht.

In diesem Buch gehe ich folgender Frage nach: Welcher Zusammenhang besteht zwischen dem Handeln traditioneller Autoritäten und Konflikten um Zugang zu Land im Kontext von land grabbing? Ziel ist es, empirisch begrün- 
detes Wissen zur Erklärung von Konflikten, die im Zusammenhang mit land grabbing in traditionellen Landverwaltungssystemen auftreten, zu schaffen. Empirisch vergleiche ich zwei Konflikte um Zugang zu Land in der Ashanti und der Northern Region Ghanas, die im Kontext von land grabbing entstanden sind. Für meine Analyse erarbeite ich einen theoretischen Rahmen, der ein differenziertes Verständnis von Zugang zu Land in traditionellen Landverwaltungssystemen mit Steven Lukes' (2005) Konzept der drei Dimensionen von Macht kombiniert.

Anhand meiner Analyse zeige ich, wie sich für unterschiedliche Akteure der Zugang zu Land im Zusammenhang mit land grabbing verändert und welche zentralen Widersprüche die damit verbundenen Konflikte strukturieren. Ich komme zu dem Ergebnis, dass land grabbing mit einer Zentralisierung der Kontrolle über den Zugang zu Land zugunsten bestimmter traditioneller Autoritäten einhergeht. Anhand von Lukes' Machtkonzept identifiziere ich typische Handlungsstrategien, über die es diesen traditionellen Autoritäten gelingt, sich die Kontrolle über den Zugang zu Land anzueignen. Ich zeige, wie sich diese Handlungsstrategien auf traditionelle Herrschaftssysteme sowie auf die Entstehung und den Verlauf von Konflikten um Zugang zu Land auswirken. 


\section{Einleitung}

Im Spannungsfeld zwischen globaler Finanz-, Rohstoff- und Nahrungsmittelpreiskrise sowie dem Klimawandel und seinen möglichen Auswirkungen beobachten Wissenschaftler*innen seit Mitte der 200oer Jahre eine zunehmende Nachfrage nach Land (Borras/Franco 2012). Zyklisch steigende Energieund Nahrungsmittelpreise, ein erhöhter Bedarf an Rohstoffen, Mineralien und Agrarkraftstoffen sowie die Notwendigkeit neuer Kapitalanlagemöglichkeiten haben Land zu einem gefragten Investitions- und Spekulationsobjekt gemacht (Akram-Lodhi 2012). Ausdruck für diese Entwicklung ist die $\mathrm{Zu-}$ nahme großflächiger Aneignungen von Land durch nationale, internationale und multinationale Akteure wie Unternehmen, Regierungen oder lokale Eliten (Dietz/Engels 2020). Typische Ziele dieser Landaneignungen sind neben der Spekulation die kommerzielle Nahrungsmittel-, Futtermittel- und Agrarkraftstoffproduktion, die Kommodifizierung der Senkenfunktion von Wäldern, Naturschutzmaßnahmen oder der Abbau von Mineralien und Rohstoffen (Borras et al. 2016; Cotula 2012; Edelman et al. 2013; Hall et al. 2015; Schoneveld/German 2014). Für landwirtschaftliche Zwecke allein belaufen sich die seit dem Jahr 2000 vertraglich abgesicherten Landaneignungen laut Nolte et al. (2016) weltweit auf eine Fläche von 26,7 Millionen Hektar. Sie konzentrieren sich vor allem in Afrika, Asien, Lateinamerika und Südosteuropa, wobei der afrikanische Kontinent mit 10 Millionen Hektar flächenmäßig am stärksten von dieser Entwicklung betroffen ist (ebd.: 16). Dort eignen sich Investoren insbesondere gewohnheitsrechtlich verwaltetes und kollektiv genutztes Land an (Dell'Angelo et al. 2017). In diesem Buch bezeichne ich das gegenwärtige Phänomen der flächen- und zahlenmäßig zunehmenden Aneignungen von Land als land grabbing. Land grabbing verstehe ich in Anlehnung an Borras et al. (Borras/Kay/et al. 2012: 405) als Aneignung der Kontrolle über relativ große Flächen an Land und anderen Ressourcen, die mit einer intendierten Inwertsetzung einhergeht und eine Verlagerung weg von der kleinbäuerli- 
chen, subsistenzorientierten Landwirtschaft hin zu einer agrarindustriellen, marktorientierten Landwirtschaft mit sich bringt. Mit der Wahl dieses Terminus grenze ich mich von synonym verwendeten Bezeichnungen wie Investitionen in oder Transaktionen von Land $a b$ und rücke insbesondere die Veränderung von Kontrollverhältnissen in den Fokus.

Land grabbing geht in vielen Fällen mit tiefgreifenden Veränderungen der Landnutzung und der Landbesitzverhältnisse einher. In diesem Zusammenhang entstehen zunehmend Konflikte, die auf unterschiedlichen Ebenen und zwischen unterschiedlichen Akteuren verlaufen (Borras/Franco 2013; Engels/Dietz 2017; Peters 2013a). Diese Konflikte treten typischerweise in drei Akteurskonstellationen auf: zwischen Landnutzer*innen und Unternehmen, Landnutzer"innen und staatlichen Akteuren sowie zwischen unterschiedlichen Gruppen von Landnutzer*innen (Borras/Franco 2013). Zentrale Themen in diesen Konflikten sind die Ausbeutung, Vertreibung und Enteignung von Landnutzer"innen oder Landbewohner*innen durch land grabbing, antagonistische Interessen sozial differenzierter Gruppen bezüglich der geplanten Vorhaben, die Konkurrenz von unterschiedlichen Landnutzer"innengruppen um verbleibende Nutzflächen sowie die Zerstörung und Verschmutzung der Umwelt (Borras/Franco 2013). Das verbindende Element in all diesen Konflikten ist die Aushandlung von Zugang zu Land, wobei Zugang zu Land bedeutet, dass Akteure einen Nutzen aus dem Land ziehen können (Blaikie 1985; Ribot/Peluso 2003). Aus dieser Perspektive verlaufen Konflikte zwischen Akteuren, die Zugang zu Land kontrollieren, denjenigen die sich Zugang neuaneignen, und denjenigen, die Zugang über Dritte erlangen, um ihren Lebensunterhalt zu bestreiten. Je nach Ausgestaltung des Zugangs zu Land sind in den Konflikten unterschiedliche Akteure involviert.

In vielen Ländern Subsahara-Afrikas ist Zugang zu Land durch rechtsund normpluralistische Landverwaltungssysteme bestimmt, in denen traditionelle, informelle und staatliche Institutionen und Gesetze zum Tragen kommen. Diese können parallel zueinander existieren, komplementär sein, sich überlagern oder in Konkurrenz zueinander stehen (Bierschenk 2010; Lund 2006; Olivier de Sardan 2011; Ubink 2008b). Rechts- und normpluralistische Strukturen in der Landverwaltung sind historisch spezifisch, dynamisch und nicht per se konfliktiv. Wenn in diesen Kontexten jedoch Konflikte um Land auftreten, stellen die unterschiedlichen rechtlichen und normativen Grundlagen einen wichtigen Ausgangspunkt für die Analyse dieser Konflikte dar (Bierschenk/Olivier de Sardan 1997; Boamah 2014a; Lund 1998, 2006). 
Schätzungsweise 70 Prozent aller land- und viehwirtschaftlichen Nutzflächen in Subsahara-Afrika unterliegen der Verwaltung im Rahmen von Gewohnheitsrechten. Diese haben, im Gegensatz zu staatlichem Recht, ihren Ursprung nicht in der staatlichen Politik. Dennoch sind Gewohnheitsrechte in vielen Ländern gesetzlich anerkannt oder zumindest, mangels Alternativen akzeptiert. Die gewohnheitsrechtliche Verwaltung von Land erfolgt durch traditionelle Institutionen. Als traditionelle Institutionen bezeichne ich historisch und sozial generierte und verinnerlichte Verhaltenserwartungen, und -weisen, die sich als grundlegende Struktur durch das jeweilige System ziehen und soziale Interaktion regeln. Sie ermöglichen oder beschränken das Handeln sozialer Akteure. Typischerweise sind diese Institutionen informell. Regeln und Verfahrensweisen sind nicht verschriftlicht und treten nicht über »offizielle«, staatliche Wege in Kraft, sondern erlangen ihre Geltung durch Autolizenzierung und hängen in ihrem Fortbestand von ihrer faktischen Anerkennung ab (Helmke/Levitsky 2004: 727). »Traditionell« bezieht sich hier auf Institutionen die im Gegensatz zu den Institutionen des postkolonialen Staates ein gewisses kulturelles Erbe abbilden, das aus der Gesellschaft selbst hervorgegangen ist (Englebert 2002a: 366), unabhängig davon, ob dieses Erbe wahr, erfunden oder ausgedacht ist (vgl. Ranger 1983). »Traditionell« bedeutet somit nicht, praktizierte Bräuche stammten aus einer unberührten präkolonialen Vergangenheit, seien zeitlos, unveränderbar und anachronistisch. Ich schließe mich dieser Auffassung an und grenze mich von einem Verständnis von Tradition als mangelnde Modernität oder als Relikt aus der Vergangenheit, das es zu überwinden gilt, ab. Vielmehr gehe ich davon aus, dass sich Tradition im Zusammenhang mit gesellschaftlichem, wirtschaftlichem und politischen Wandel entwickelt und verändert. Das erklärt auch die unterschiedliche Ausgestaltung des Gewohnheitsrechts in seiner zeitlichen und räumlichen Dimension. Gewohnheitsrechte unterscheiden sich von Dorf zu Dorf, von Region zu Region und von Staat zu Staat.

In traditionellen Herrschaftssystemen liegt die Verwaltung von Land maßgeblich in der Verantwortung traditioneller Autoritäten, die sich ebenso wie die traditionellen Systeme selbst - durch eine große Vielfalt auszeichnen (von Trotha 1996: 80). Diese Vielfalt bezieht sich unter anderem auf das Verhältnis traditioneller Autoritäten zu staatlichen Akteuren, auf ihre Legitimationsgrundlage, ihre Ressourcen, Handlungsspielräume und ihre Zuständigkeitsbereiche (Ubink 2008c). Unterschiedliche Bezeichnungen für traditionelle Autoritäten tragen dieser Vielfalt Rechnung. Im englischsprachigen Raum finden häufig die Termini chiefs und traditional authorities 
Verwendung, es existieren jedoch auch Bezeichnungen wie clan head, family head, headman, queen mother, spiritual leader oder earth priest. Das Pendant dazu im französischsprachigen Raum sind die chefs de village, chefs de terre und chef coutumiers. Da es im Deutschen keine adäquate Übersetzung für diese Begriffe gibt, verwende ich im Folgenden die Bezeichnungen chief und traditionelle Autorität synonym. Sie können sich sowohl auf weibliche als auch auf männliche Titelträger*innen beziehen. Unabhängig von ihrer Bezeichnung ist allen traditionellen Autoritäten gemein, dass ihre Anerkennung, Legitimität und Macht auf ihrer Zugehörigkeit zu einer ortsbezogenen Gemeinschaft beruhen, über die sie unter Berücksichtigung gewohnheitsrechtlicher Bräuche herrschen (Baldwin 2016: 21).

In vielen Ländern - wie in Ghana, Mali, Zimbabwe, Malawi, oder Ugan$\mathrm{da}$ - sind traditionelle Autoritäten die zentralen Entscheidungsträger*innen bezüglich der Vergabe von gewohnheitsrechtlich verwaltetem Land an kleinbäuerliche Nutzer*innen (Logan 2013). Lange Zeit waren Gewohnheitsrechte von staatlicher Seite aus eher geduldet als tatsächlich anerkannt. Seit den 1990er Jahren gibt es jedoch eine Tendenz zur staatlichen Anerkennung dieser Rechte (Knierzinger 2011; Ubink 2008c). Gleichzeitig hat sich durch Landrechtsreformen, Dezentralisierungs- und Strukturanpassungsprogramme sowie die Liberalisierung der Marktwirtschaft seit den frühen 1990er Jahren auch die rechtliche Ausgestaltung und Strukturierung des Landsektors in Ländern des Globalen Südens ${ }^{1}$ verändert. In diesem Zusammenhang haben traditionelle Autoritäten ebenfalls an Einfluss in der Landverwaltung gewonnen (Ubink 2008c). In jüngster Zeit treten sie im Kontext von land grabbing als zentrale Akteure in der großflächigen Vergabe von Land an Investoren in Erscheinung (Ahmed et al. 2018; Capps 2016, 2018). Den Begriff der großflächigen Vergabe von Land nutze ich synonym zu land grabbing, hebe dadurch aber die Beteiligung traditioneller Autoritäten hervor. Die Tatsache, dass land grabbing oftmals mit Konflikten um den Zugang zu Land einhergeht, macht traditionelle Autoritäten für die Analyse von Konflikten um Zugang

1 Die Bezeichnungen Globaler Süden und Globaler Norden verweisen nicht auf die geographische Lage von Staaten, sondern auf Ungleichheitsverhältnisse in den weltpolitischen und wirtschaftlichen Positionen der Länder sowie auf unterschiedliche Erfahrungen mit Kolonialismus und Ausbeutung. Der Begriff Globaler Süden bezieht sich nicht auf einzelne Nationalstaaten, sondern auf die Gesamtheit der marginalisierten Bevölkerung (de Sousa Santos 2016: 19). Der Globale Norden umfasst im Gegenzug all diejenigen, die im globalen Machtgefüge eine privilegierte Position einnehmen (glokal 2013: 8). 
in diesem Zusammenhang zu Schlüsselfiguren. Welche Rolle traditionelle Autoritäten in diesen Konflikten spielen, ist bislang jedoch kaum erforscht. In diesem Buch gehe ich folgender Forschungsfrage nach: Welcher Zusammenhang besteht zwischen dem Handeln traditioneller Autoritäten und Konflikten um Zugang zu Land im Kontext von land grabbing? Im Zentrum des Erkenntnisinteresses stehen drei Fragen: (1) Wie verändert sich Kontrolle über den Zugang zu Land im Kontext von land grabbing? (2) Wie verändern sich in diesem Zusammenhang traditionelle Herrschaftssysteme? Und (3) wie wirkt sich das Handeln traditioneller Autoritäten auf die Entstehung und den Verlauf von Konflikten um Zugang zu Land aus?

\subsection{Problemstellung}

Ausgangspunkt meiner Forschung ist die Beobachtung, dass in rechts- und normpluralistischen Landverwaltungssystemen Subsahara-Afrikas traditionelle Autoritäten im Kontext von land grabbing zunehmend als Verhandlungspartner"innen von Investoren für die Vergabe von Land verantwortlich sind (Boamah 2014a: 407; Lanz et al. 2018; Yaro/Tsikata 2014). Aktuelle Studien belegen, dass im Zusammenhang mit land grabbing oftmals Konflikte auftreten und dass traditionelle Autoritäten aufgrund ihrer Zuständigkeit für die Vergabe von Land zentrale Akteure in diesen Konflikten sind, in denen sie gleichzeitig auch als Mediator*innen agieren (Campion/Acheampong 2014). Darüber hinaus gibt die bestehende Literatur zu Konflikten, die im Zusammenhang mit land grabbing auftreten, wenig Aufschluss darüber, welche Rolle traditionelle Autoritäten in diesen Konflikten spielen, wie sich traditionelle Herrschaftssysteme in Verbindung mit land grabbing verändern und warum in diesem Zusammenhang Konflikte auftreten. In Bezug auf Akteurskonstellationen konzentrieren sich Analysen zu land grabbing und Konflikten bislang vornehmlich auf die Rolle des Staates (Busscher et al. 2019; Hall et al. 2015; Wolford et al. 2013), von Unternehmen (Oya 2013; Scoones et al. 2013) und sozialen Bewegungen (Engels 2019; Prause 2019). Auch wissenschaftliche Arbeiten zu traditioneller Herrschaft geben keinen Aufschluss zu traditionellen Autoritäten als Konfliktakteure, sondern interessieren sich insbesondere für ihre Rolle als Intermediäre, Entwicklungsagent*innen (Bierschenk et al. 2000; Kleist 2011; Knierzinger 2011) und Mediator"innen (Rukuni et al. 2015; Santschi 2014; Tubiana et al. 2012). Die aktuelle Forschung gibt keinen Aufschluss darüber, wie sich traditionelle Herrschaft im Kontext von land grabbing ver- 
ändert, inwiefern sich mögliche Veränderungen auf den Zugang zu Land von Landnutzer*innen auswirken und warum in diesem Kontext Konflikte entstehen. Konflikte verstehe ich als soziale Beziehungen zwischen mindestens zwei individuellen oder kollektiven Akteuren gekennzeichnet durch von ihnen als widersprüchlich wahrgenommene Interessen, Ziele oder Bedürfnisse (Bonacker/Imbusch 2006). Traditionelle Autoritäten gelten als Repräsentant*innen ihrer Gemeinschaft und ihre Aufgabe ist es, die Interessen dieser Gemeinschaft zu vertreten. Wie erklären sich also zunehmende Widersprüche und damit einhergehende Konflikte in traditionellen Landverwaltungssystemen im Zusammenhang mit land grabbing, und welchen Einfluss haben traditionelle Autoritäten auf die Entstehung und den Verlauf dieser Konflikte?

Ziel meiner Forschung ist, es empirisch begründetes Wissen zur Erklärung von Konflikten um Zugang zu Land, die in traditionellen Landverwaltungssystemen im Zusammenhang mit land grabbing auftreten, zu generieren. Meine erste Annahme diesbezüglich ist, dass das Handeln traditioneller Autoritäten als Schlüsselfiguren in der Vergabe von Land zentral für die Entstehung und den Verlauf dieser Konflikte ist. Der Gegenstand meiner Analyse ist daher das Handeln traditioneller Autoritäten. Diesbezüglich beziehe ich mich auf relationale Erklärungsansätze von Konflikten. Diesen zufolge können Konflikte nicht allein über das Handeln einer Person erklärt werden, sondern nur unter Berücksichtigung der Interaktion mit anderen Akteuren. Das bedeutet für meine Analyse, dass ich das Handeln traditioneller Autoritäten hinsichtlich der Interaktion mit anderen Akteuren, die in die Landvergabe einbezogen oder von ihr betroffen sind, untersuche. Meine zweite Annahme ist, dass die Ausgestaltung traditioneller Herrschaftssysteme das Handeln traditioneller Autoritäten beeinflusst, was sich wiederum auf Konflikte auswirken kann. In meinem Forschungsdesign habe ich mich daher für eine kollektive Fallstudie entschieden. Eine kollektive Fallstudie bezeichnet die Ausweitung einer Einzelfallstudie auf zwei oder mehrere Fälle (Stake 2003: 138). Die Erforschung von zwei Fällen ermöglicht mir, Unterschiede und Parallelen im Handeln traditioneller Autoritäten in unterschiedlichen Herrschaftssystemen zu untersuchen und verallgemeinerbare Hypothesen zu generieren.

Hinsichtlich der bestehenden Literatur beziehe ich mich auf Studien zu (1) Konflikten im Zusammenhang mit land grabbing, (2) Land, sozialer Zugehörigkeit und Konflikten und (3) traditionellen Autoritäten als Intermediäre. Der Bezug auf diese drei Forschungsfelder ist aus folgenden Gründen für die Bearbeitung meiner Fragestellung sinnvoll. Beiträge zu Konflikten im Kontext von land grabbing liefern Anhaltspunkte dazu, warum diese Konflikte entste- 
hen, welche übergeordneten Fragen Akteure in diesen Konflikten verhandeln und welche Akteure in diesen Konflikten typischerweise handeln. Die Forschung zu Land, sozialer Zugehörigkeit und Konflikten rückt die Aushandlung von Zugang zu Land in traditionellen Landverwaltungssystemen in den Fokus. Beiträge zu diesem Thema widmen sich insbesondere der Bedeutung sozialer Zugehörigkeit und Differenzierung hinsichtlich der Aushandlung von Zugang zu Land in traditionellen Landverwaltungssystemen und geben Aufschluss darüber, welche Wechselwirkung dort zwischen Machtverhältnissen und der Kontrolle des Zugangs zu Land besteht. Für meine Forschung, in der traditionelle Landverwaltungssysteme den Kontext bilden, sind diese Beiträge eine sinnvolle Ergänzung der Literatur zu land grabbing und Konflikten. Das dritte Literaturfeld zu traditionellen Autoritäten als Intermediäre gibt Aufschluss über die geschichtliche Entwicklung der Rolle traditioneller Autoritäten im Spannungsfeld gesellschaftlichen, politischen und wirtschaftlichen Wandels und ermöglicht ein differenziertes Verständnis der möglichen Herausforderungen und Widersprüche, die sich aus dem Handeln traditioneller Autoritäten ergeben können.

In der empirischen Analyse identifiziere ich typische Handlungsstrategien traditioneller Autoritäten in der Landvergabe an Investoren in unterschiedlichen traditionellen Herrschaftssystemen. Ich untersuche, inwiefern sich diese Handlungsstrategien auf den Zugang zu Land von unterschiedlichen Landnutzer*innen sowie auf die Entstehung und den Verlauf von Konflikten auswirken. Die gewonnenen Erkenntnisse bilden die Grundlage für die Entwicklung von Thesen, die der Erweiterung theoretischer Zugänge zu Konflikten um Zugang und ihrer sozio-politischen Vermittlung in traditionellen Landverwaltungssystemen dienen können.

\subsection{Forschungskontext und Forschungsdesign}

Während der Kolonialzeit galten traditionelle Autoritäten als der verlängerte Arm der Kolonialverwaltung. Grund dafür war ihre der administrative chiefs, die sie in vielen Ländern Subsahara-Afrikas innehatten (Mamdani 1996: 52-61; von Trotha 1996). Entsprechend niedrig war ihre Beliebtheit bei den sich neu formierenden, nach Unabhängigkeit strebenden nationalen Regierungen. Das Bild traditioneller Autoritäten als Kollaborateur*innen der Kolonialmächte hatte auch nach der Unabhängigkeit der Kolonialstaaten Bestand. Es gab allerdings Unterschiede in der Haltung der postkolonialen Regierungen 
gegenüber traditionellen Autoritäten. Viele standen traditionellen Autoritäten aufgrund iherer geringen Beteiligung an den Unabhängigkeitskämpfen weiterhin kritisch gegenüber (Baldwin 2016: 32). In einer Ära der Demokratisierung galten traditionelle Autoritäten zudem als antidemokratische lokale Despoten (Baldwin 2016: 3). Postkoloniale Regierungen nahmen sie als Konkurrent*innen oder als Bedrohung ihrer Macht wahr. Über unterschiedliche Wege versuchten sie den Einfluss traditioneller Autoritäten in der Lokalverwaltung und nationalen Politik zu beschränken. Typische Maßnahmen waren die formelle Abschaffung traditioneller Herrschaftssysteme, die Beschränkung der Handlungsmöglichkeiten traditioneller Autoritäten in zentralen Bereichen wie Bildung, Gesundheit und Landverwaltung oder die Ignoranz traditioneller Herrschaft. Letztere konnte ebenso zu einer Wiederbelebung wie auch zum Bedeutungsverlust traditioneller Herrschaft führen (Ubink 2008c: 10; von Trotha 2000: 257). Insgesamt verschwand sie jedoch nicht. Im Gegenteil: Mehr als 50 Jahre nach den ersten Unabhängigkeitserklärungen wird deutlich, dass die meisten Versuche, traditionelle Herrschaft durch staatliche Institutionen $\mathrm{zu}$ ersetzen, scheiterten. Zahlreiche Wissenschaftler*innen zeigen in ihren Forschungen, dass traditionelle Autoritäten eine erstaunliche Anpassungsfähigkeit an soziopolitischen Wandel unter Beweis stellen und weiterhin in der Lage sind, Einfluss in zentralen Bereichen wie der Lokalverwaltung, Konfliktschlichtung oder Landverwaltung auszuüben (Baldwin 2016; Berry 1992; van Rouveroy van Nieuwaal 1996: 45). Seit den 1990er Jahren interessieren sich afrikanische Regierungen und internationale Geberorganisationen wieder zunehmend für traditionelle Autoritäten (Ubink 2008c: 11) und Wissenschaftler*innen sprechen von einer »Wiederbelebung« traditioneller Herrschaft (Bräuchler/Widlok 2007; Buur/Kyed 2007; Englebert 2002b). In Ländern wie Uganda, Mosambik, Südafrika und Ghana - in denen traditionelle Herrschaft zwar nach der Unabhängigkeit nicht formell abgeschafft wurde, jedoch deutliche Restriktionen erfuhr - ging diese Wiederbelebung sogar mit einer verfassungsrechtlichen Formalisierung des chieftaincy-Systems einher (Ubink 2008c).

Der Bedeutungsgewinn traditioneller Herrschaft steht auch im Zusammenhang mit der zunehmenden Kritik von Geberorganisationen und der internationalen Entwicklungspolitik an der institutionellen Qualität in vielen afrikanischen Staaten, insbesondere auf lokaler Ebene. Die schwachen Kapazitäten und die mangelnde Leistungsfähigkeit lokaler staatlicher Institutionen machte etwa die Weltbank für die vermeintlich schlechte wirtschaftliche Entwicklung verantwortlich (Brautigam 1992; Hydén/Bratton 1992; World 
Bank 1989, 1992, 1994). Die Kooperation mit traditionellen Autoritäten stellte für die Weltbank einen möglichen Lösungsansatz für dieses Problem dar (World Bank 2002). Die gezielte Einbindung traditioneller Autoritäten in die Lokalpolitik, zum Beispiel im Rahmen von Dezentralisierungsbestrebungen, sollte die Regierungsführung auf lokaler Ebene stärken und fehlende staatliche Kapazitäten ausgleichen. Hinzu kam, dass chiefs sich bei mangelnder Einbeziehung in vielen Fällen der Umsetzung staatlicher Politik auflokaler Ebene widersetzten und angestrebte Maßnahmen dadurch scheiterten (Stacey 2015).

Die Kooperation mit traditionellen Autoritäten steht in Einklang mit einer dem Selbstanspruch nach partizipatorischen Entwicklungspolitik (OECD/DAC 1996: 9). Vor diesem Hintergrund gewannen traditionelle Autoritäten in Subsahara-Afrika an Prominenz. In der entwicklungspolitischen Praxis erlangten sie als Intermediäre an Bedeutung. Wissenschaftliche Studien verhandeln diese neue Rolle unter den Bezeichnungen Entwicklungspartner*innen (agents of development) und Entwicklungsvermittler*innen (development broker) (Baldwin 2016: 69ff.; Grischow 2008; Knierzinger 2011; Yaro/Tsikata 2014). Als Intermediäre zwischen internationalen Geber*innen und lokalen Empfänger*innen sind sie für die Vernetzung von Entwicklungsakteuren und der lokalen Gemeinschaft, eine bessere sozio-kulturelle Verständigung, die Verwaltung von Projektgeldern, die Mobilisierung von Arbeitskräften und die Mediation in Konflikten zuständig (Knierzinger 2011: 28f.; Ubink 2008a: 141ff.). Darüber hinaus treten traditionelle Autoritäten in zentralen Bereichen wie dem Bildungs- oder Gesundheitssektor, in denen die Kapazitäten staatlicher Institutionen schwach sind, als Dienstleister*innen auf (Ubink 2008c: 14). Durch diese Tätigkeit steigen ihr Ansehen und ihre Legitimität innerhalb der Gemeinschaft, was mit der einer Stärkung ihrer gesellschaftlichen Stellung und der Ausweitung ihrer Macht einhergeht. Die Ausweitung ihrer Macht wiederum ermöglicht ihnen, Entscheidungen zu treffen, ohne ihrer Gemeinschaft oder staatlichen Akteuren gegenüber Rechenschaft ablegen $\mathrm{zu}$ müssen. So sind sie in der Lage, ihre Interessen durchzusetzen, auch wenn diese im Widerspruch zu den Interessen der Gemeinschaft stehen. Ein Beispiel dafür ist die Selbstbereicherung einiger chiefs an gemeinschaftlich genutzten Ressourcen wie Land (Ubink 2008c: 27).

Hinsichtlich der zentralen Bedeutung traditioneller Autoritäten in der Vergabe von Land an Investoren ist die Erkenntnis, dass traditionelle Autoritäten sich vermehrt selbst an gemeinschaftlich genutzten Ressourcen bereichern, von besonderer Relevanz. Sie lenkt den Blick auf die widersprüchlichen Rollen traditioneller Autoritäten. Sie sind Repräsentant*innen der Gemein- 
schaft und zuständig für Dienstleistungen, wie die Vergabe von Land oder den Ausbau des Gesundheits- und Bildungssektors. Gleichzeitig sind sie auch Akteure mit eigenen Interessen, die teilweise im Widerspruch zu den Interessen ihrer Gemeinschaft stehen. Das kann ein Ausgangspunkt für Konflikte sein.

Um untersuchen zu können, welcher Zusammenhang zwischen dem Handeln traditioneller Autoritäten und Konflikten um Zugang im Zusammenhang mit land grabbing besteht, entwickle ich einen Analyserahmen, der ein theoretisches Verständnis von Zugang $\mathrm{zu}$ Land in traditionellen Landverwaltungssystemen mit einem auf die empirische Analyse ausgerichteten mehrdimensionalen Machtkonzept verbindet. In traditionellen Landverwaltungssystemen erlangen Landnutzer*innen Zugang zu Land nicht ausschließlich über Besitz, sondern auch über eine Vielzahl weiterer rechtebasierter, relationaler und struktureller Mechanismen, zu denen verschiedene traditionelle Rechte, individuelle Vereinbarungen und persönliche Beziehungen zählen. Aufgrund meines Verständnisses von Konflikten im Zusammenhang mit land grabbing als Konflikte um Zugang zu Land gehe ich davon aus, dass die Frage, über welche Mechanismen Zugang zu Land erlangt wird, wichtig für die Erklärung dieser Konflikte ist. Ein theoretisches Verständnis von Zugang zu Land in traditionellen Landverwaltungssystemen soll ermöglichen zu erfassen, über welche Mechanismen Landnutzer*innen Zugang zu Land erlangen und inwiefern sich ihre Möglichkeiten auf diese Mechanismen zurückzugreifen im Kontext der großflächigen Landvergabe verändern. Mein theoretisches Verständnis von Zugang zu Land in traditionellen Landverwaltungssystemen entwickle ich auf der Grundlage der Theory of Access im Anschluss an Jesse Ribot und Nancy Peluso (Ribot/Peluso 2003). Diese erweitere ich durch Erkenntnisse aus der Forschung zu Zugang zu Land in traditionellen Landverwaltungssystemen Subsahara-Afrikas. Über die Analyse der Veränderung des Zugangs zu Land arbeite ich die zentralen Widersprüche heraus, die sich durch die großflächige Landvergabe ergeben und sich in Konflikten um Zugang manifestieren. Um zu verstehen, in welchem Zusammenhang das Handeln traditioneller Autoritäten mit Konflikten um Zugang steht, fehlt ein erklärendes Element. Dieses erklärende Element soll ermöglichen zu analysieren, wie es Konfliktakteuren - und insbesondere den für die Landvergabe zuständigen traditionellen Autoritäten - gelingt ihren Zugang zu Land und den Zugang Dritter im Kontext der großflächigen Landvergabe aufrechtzuerhalten oder zu kontrollieren. Viele Autor*innen betonen, dass die Fähigkeit Kontrolle über den Zugang zu Land auszuüben 
eine Frage von Machtverhältnissen ist (Bryant/Bailey 1997; Peluso/Lund 2011; Peluso/Ribot 2020). In welcher Form Akteure Macht ausüben, um Zugang $\mathrm{zu}$ Land zu kontrollieren bleibt jedoch unklar. Daher kombiniere ich in meinem Analyserahmen das theoretische Verständnis von Zugang zu Land in traditionellen Landverwaltungssystemen mit Steven Lukes' Konzept der drei Dimensionen von Macht (Lukes 2005). Dieses Konzept berücksichtigt Macht auf unterschiedlichen Ebenen: auf der Ebene (individueller) Entscheidungen, der Ebene institutioneller Verfahrensweisen sowie der Ebene gesellschaftlicher Kräfteverhältnisse. Das Konzept bietet sich für meine Forschung an, da es durch die Berücksichtigung unterschiedlicher Formen von Macht eine Offenheit gegenüber empirischen Phänomenen erlaubt. Damit eignet es sich für explorative Forschungsdesigns wie das meiner Studie, die darauf abzielen, neue Erkenntnisse zu bislang wenig erforschten Phänomenen zu gewinnen. Mithilfe dieses Konzepts untersuche ich, inwiefern traditionelle Autoritäten im Rahmen der Landvergabe Macht ausüben, um den Zugang $\mathrm{zu}$ Land kontrollieren zu können. Über die Untersuchung identifiziere ich typische Handlungsstrategien, über die traditionelle Autoritäten Zugang $\mathrm{zu}$ Land kontrollieren, und zeige, wie sich traditionelle Herrschaftssysteme dadurch im Kontext von land grabbing verändern. Anschließend analysiere ich, inwiefern sich diese Handlungsstrategien auf die Entstehung und den Verlauf von Konflikten auswirken.

\subsection{Fallauswahl}

In der empirischen Forschung konzentriere ich mich auf Ghana. Folgende vier Faktoren waren für die Auswahl dieses Fallstudienlands ausschlaggebend: erstens die Relevanz traditioneller Autoritäten in den Bereichen Landverwaltung und Konfliktmanagement, die in Konflikten um Zugang zu Land im Zusammenhang mit land grabbing zu der zuvor beschriebenen widersprüchlichen Position traditioneller Autoritäten führt; zweitens die unterschiedliche Ausgestaltung traditioneller Herrschaft innerhalb eines Länderkontexts; drittens das Ausmaß von land grabbing und viertens die Existenz zahlreicher Konflikte, die im Zusammenhang mit land grabbing auftraten, in denen traditionelle Autoritäten zu den Konfliktakteuren gehören. Die letzten beiden Faktoren sind eine notwendige Voraussetzung für die Identifizierung geeigneter Fälle. 
In Bezug auf die Verknüpfung von Landvergabe und Konfliktmanagement ist der Einfluss traditioneller Autoritäten in Ghana besonders groß. Die bis heute starke Stellung traditioneller Autoritäten in Ghana lässt sich durch unterschiedliche Faktoren erklären. Ghana zählt, neben Mosambik und Südafrika, zu den Ländern, in denen die post-kolonialen Regierungen die Stellung traditioneller Autoritäten ab den frühen 1980er Jahren gestärkt und formalisiert haben (Ubink 2008c: 11). Seit 1992 sind traditionelle Autoritäten und das chieftaincy-System in Ghana formal durch die Verfassung anerkannt (vgl. Kasanga/Kotey 2001). Zu Beginn der 2000er Jahre konnten traditionelle Autoritäten in Ghana die Weltbank und andere Geberorganisationen davon überzeugen, als Intermediäre zwischen lokaler und (inter)nationaler Ebene eine wichtige Rolle in der Umsetzung von Entwicklungsprojekten und Verwaltungsreformen einnehmen zu können. Für andere afrikanische Staaten mit starken traditionellen Herrschaftssystemen hatten sie eine Vorbildfunktion und trugen zur Wiederbelebung traditioneller Herrschaft in SubsaharaAfrika von internationaler Seite aus bei (Berry 2013: 38; World Bank 2002).

Traditionelle Herrschaft unterscheidet sich zwischen den Regionen Ghanas. Unterschiede in der traditionellen Herrschaft sind auf die unterschiedliche Einbeziehung traditioneller Autoritäten während der Kolonialzeit zurückzuführen. Infolgedessen ist traditionelle Herrschaft in der Ashanti Region stark hierarchisch organisiert, mit besonders mächtigen chiefs an der Spitze. In anderen Regionen ist traditionelle Herrschaft stärker durch Clanund Familien-Strukturen bestimmt oder eng mit spirituellen Aufgaben verknüpft. In meiner Forschung gehe ich davon aus, dass die unterschiedliche Ausgestaltung traditioneller Herrschaft ein möglicher Ansatzpunkt für die sozio-politische Vermittlung von Konflikten, die im Zusammenhang mit land grabbing auftreten, ist. Neben den bisher erläuterten relevanten Faktoren für die Fallauswahl eignet sich Ghana besonders als Fallstudienland, da es sich seit Beginn der $2000 e r$ Jahre zu einem beliebten Zielland für die Umsetzung großflächiger inter- und multinationaler agrarindustrieller Projekte entwickelt hat (Schoneveld/German 2014). Die Land Matrix verzeichnet für Ghana aktuell 100 Fälle von land grabbing durch inter- und multinationale Investoren (The Land Matrix 2020). In ihrer Erhebung berücksichtigt die Land Matrix Flächen mit einer Größe von 200 Hektar aufwärts (Nolte et al. 2016: 3). Damit liegt Ghana im Vergleich zu anderen Ländern Subsahara-Afrikas zahlenmäßig nur hinter Mosambik, Äthiopien und Tansania. Wie auch in anderen Ländern sind traditionelle Autoritäten in Ghana seit Beginn der 200oer Jahre zu wichtigen Verhandlungspartner*innen in der großflächigen Verga- 
be von Land an Investoren geworden (Cotula et al. 2014). Aufgrund der verfassungsrechtlichen Anerkennung traditioneller Herrschaft und der uneingeschränkten Zuständigkeit traditioneller Autoritäten in der Landverwaltung haben sie relativ freie Hand in den Verhandlungen mit Investoren. Zugleich sind sie aber auch dafür zuständig, die Interessen ihrer Gemeinschaft zu vertreten. Ihnen obliegt es, die Zugangsrechte der Landnutzer*innen zu schützen, im Fall des Verlusts von Land angemessene Entschädigungszahlungen durchzusetzen, Kleinbäuerinnen und -bauern in die Verhandlungen miteinzubeziehen und ihnen die Möglichkeit des Widerspruchs oder der Überprüfung von Vereinbarungen zu geben (Elhadary/Obeng-Odoom 2012; Vermeulen/Cotula 2010: 907). Dieser Aufgabe kommen viele von ihnen nicht nach, sondern handeln aus Eigeninteresse. Wie auch in anderen Ländern treten im Verlauf der Verhandlungen und während der Implementierung der Projekte in Ghana häufig Konflikte auf: Konflikte zwischen unterschiedlichen Gruppen von Landnutzer*innen, Konflikte, die durch die unrechtmäßige Vergabe von Land oder die Intransparenz der Verhandlungen entstehen, und Konflikte zwischen unterschiedlichen Nutzer*innengruppen wie Kleinbäuerinnen und -bauern und mobilen Viehzüchter*innen (Tsikata/Yaro 2011). Aufgrund ihrer zentralen, aber auch ambivalenten Rolle in der Vergabe großer Flächen an Land zählen traditionelle Autoritäten in Ghana zunehmend zu den Konfliktakteuren. Welche Rolle sie in diesen Konflikten einnehmen, ist in der Forschung bislang kaum untersucht worden (Boamah 2014a: 407).

Ich analysiere das Handeln traditioneller Autoritäten in Konflikten um großflächige Landtransaktionen in Ghana anhand eines Vergleichs von zwei Fällen: die Konflikte um das ScanFarm-Projekt in Ashanti und das BioFuel Africa-Projekt in der Northern Region. Beide Fälle sind Konflikte um agrarindustrielle Vorhaben, die ursprünglich den Anbau von Jatropha (bot. jatropha curcas) anstrebten. Jatropha ist eine strauchige Pflanze, deren Samen stark ölhaltig sind. Das aus ihnen gewonnene Öl dient der Produktion von Agrarkraftstoffen. Der großflächige Anbau von Energiepflanzen - und insbesondere von Jatropha - mit dem Ziel der Agrarkraftstoffproduktion hat sich vielerorts als besonders konfliktreich herausgestellt. Ab Mitte der 200oer Jahre kam es in vielen Ländern Subsahara-Afrikas zu einem regelrechten JatrophaBoom, von dem riesige Flächen Ackerland betroffen waren und dem in der wissenschaftlichen Debatte um land grabbing ein hohes Maß an Aufmerksamkeit zuteilwurde - nicht zuletzt aufgrund der vielfältigen Konflikte, die er mit sich brachte (Campion/Acheampong 2014; Hunsberger 2010; Schrader 2014; von Maltitz et al. 2014). Mittlerweile ist dieser Boom vorüber und die meisten 
Unternehmen konzentrieren sich auf den Anbau anderer Kulturpflanzen, insbesondere so genannter flex crops wie Mais, Soja, Ölpalmen oder Zuckerrohr, die je nach Nachfrage und Marktpreis flexibel als Nahrungs- oder Futtermittel sowie zur Herstellung von Kosmetika und Agrarkraftstoffen dienen können (Borras et al. 2016). Die Konflikte bestehen jedoch in vielen Fällen weiterhin. Die räumliche Ausbreitung des Jatropha-Anbaus variierte innerhalb Ghanas und betraf vor allem die drei nördlichen Regionen (Northern Region, Upper East, Upper West) sowie Brong-Ahafo und Ashanti ${ }^{2}$ (Yirrah 2012).

Da ich die Annahme vertrete, dass die unterschiedliche Ausgestaltung traditioneller Herrschaftssysteme relevant für das Handeln traditioneller Autoritäten ist, war ein weiteres Kriterium meiner Fallauswahl die Varianz traditioneller Herrschaft innerhalb des gleichen Länderkontexts. In der Ashanti Region im Süden von Ghana sind traditionelle Autoritäten besonders mächtig. Sie sind in stark hierarchischen Strukturen organisiert, mit dem einflussreichen Asantehene, dem König der Ashanti, an der Spitze. Darüber hinaus verfügen sie über sehr gute nationale und internationale Kontakte in Wirtschafts-, Politik- und Gebernetzwerke. In den drei nördlichen Regionen ist die traditionelle Herrschaft fragmentierter als in der Ashanti Region. Ihre Ausgestaltung unterscheidet sich insofern, als dass es in jeder der Regionen mehrere kleinere chiefdoms gibt und nicht ein einziges großes Königreich wie in der Ashanti Region. Die einzelnen kleinen chiefdoms verfügen ebenfalls über eine interne Hierarchie, die vergleichbar mit derjenigen der Ashanti Region ist. Insgesamt stehen ihnen im Verhältnis weniger Ressourcen zur Verfügung, sie sind weniger gut in internationale Netzwerke eingebunden und verwalten insgesamt kleinere Flächen an Land. Hinsichtlich der Organisation des Zugangs zu Land sind die beiden Regionen vergleichbar. Die Projekte ScanFarm und BioFuel Africa wurden relativ zeitgleich von norwegischen Investoren initiiert und die ursprünglich geplante Anbaufläche hatte ein ähnliches Ausmaß. Im Kontext der Landvergabe an die Unternehmen kam es in beiden Fällen zu Konflikten, in denen traditionelle Autoritäten Verhandlungspartner und Repräsentanten der Gemeinschaft sowie Konfliktschlichter waren.

Die empirischen Daten für die Fallstudie habe ich im Verlauf von drei Feldforschungsaufenthalten in Ghana im November 2015, von Februar bis Juni 2016 und von Februar bis März 2017 erhoben. Insgesamt habe ich 46 teilstrukturierte Leitfaden-Interviews in der Hauptstadt Accra, den Regionalhauptstädten der Ashanti und Northern Region Kumasi und Tamale, 
der Hauptstadt des Asante Akyem North Districts Agogo sowie in neun von den agrarindustriellen Projekten betroffenen Dörfern geführt. Interviewpartner*innen waren Mitarbeiter*innen von Behörden auf nationaler, regionaler und Distriktebene, von NGOs (Non-Governmental Organizations) und zivilgesellschaftlichen Organisationen, leitende Angestellte der Unternehmen, Landnutzer*innen der betroffenen Dörfer, traditionelle Autoritäten und ihre Vertreter, Repräsentanten der lokalen Customary Land Secretariats (CLS) (formalisierte traditionelle Landrechtsinstitution) sowie des House of Chiefs in beiden Regionen und auf nationaler Ebene. Zusätzlich zu den Einzelinterviews habe ich sieben Gruppendiskussionen mit insgesamt 176 betroffenen Landnutzer*innen und traditionellen Autoritäten in den beiden Projektgegenden geführt.

\subsection{Struktur des Buches}

Dieses Buch ist wie folgt aufgebaut. Im Anschluss an die Einleitung stelle ich den aktuellen Stand der Forschung zu Konflikten im Kontext von land grabbing und traditionellen Autoritäten in Konflikten um Zugang zu Land dar und zeige relevante Anknüpfungspunkte für meine eigene Forschung auf. Das dritte Kapitel bildet die theoretische Grundlage der Forschung. In diesem entwickle meinen Analyserahmen und führe aus, wie sich dieser für die Analyse der empirischen Daten operationalisieren lässt. Darauf folgend erläutere ich im vierten Kapitel das Design und methodische Vorgehen meiner Forschung. Kapitel fünf dient als Überleitung zur empirischen Analyse und gibt einen Überblick über den Länderkontext Ghanas. Der Fokus dieses Kapitels liegt auf der Entwicklung von Landrechten und traditioneller Herrschaft. Kapitel sechs widmet sich der Darstellung der empirischen Fälle. Im Zentrum stehen der Ablauf der Landvergabe an die Unternehmen ScanFarm und BioFuel Afri$c a$, die im Kontext der Landvergabe auftretenden Konflikten sowie die Konfliktakteure und ihr Handeln. Anschließend analysiere ich in Kapitel sieben, inwiefern sich im Rahmen der großflächigen Vergabe von Land die Kontrolle über den Zugang zu Land verändert, und inwiefern die Veränderung von Kontrollverhältnissen den Zugang unterschiedlicher Landnutzer*innen zu Land beeinflusst. Ich untersuche, welche Akteure vor der Landvergabe Kontrolle über den Zugang zu Land ausgeübt haben, über welche Mechanismen Landnutzer*innen Zugang zu Land erlangt haben und wie dies sich im Rahmen der Landvergabe verändert hat. Aufbauend auf diesen Erkenntnissen arbeite 
ich im achten Kapitel typische Handlungsstrategien heraus, über die traditionelle Autoritäten im Rahmen der Landvergaben Macht ausüben, um den Zugang zu Land zu kontrollieren. Anschließend analysiere ich, inwiefern sich ihre Handlungsstrategien auf die Entstehung und den Verlauf von Konflikten auswirken.

Im Fazit fasse ich die Ergebnisse meiner Analyse zusammen. Ich arbeite Parallelen und Unterschiede zwischen den Fällen heraus und diskutiere, womit diese zusammenhängen. Auf der Grundlage der empirischen Erkenntnisse formuliere ich drei Hypothesen zur Erklärung von Konflikten um Zugang zu Land in traditionellen Landverwaltungssystemen und erörtere die Reichweite dieser Hypothesen. Schließlich zeige ich auf, zu welchen Forschungsfeldern ich mit diesem Buch einen Beitrag leiste. 


\section{Land grabbing, Konflikte um Land und traditionelle Autoritäten - Stand der Forschung}

Mit Blick auf die zentrale Frage, welcher Zusammenhang zwischen dem Handeln traditioneller Autoritäten und Konflikten um Zugang zu Land im Kontext von land grabbing besteht, bediene ich mich der Erkenntnisse aus drei Forschungsfeldern, zu (1) Konflikten im Zusammenhang mit land grabbing, (2) Land, sozialer Zugehörigkeit und Konflikten sowie (3) traditionellen Autoritäten als Intermediäre. In diesem Kapitel arbeite ich den aktuellen Stand der Forschung zu diesen Debatten auf, fasse die zentralen Erkenntnisse zusammen und zeige weiteren Forschungsbedarf auf.

Das Kapitel ist wie folgt strukturiert: Zunächst erarbeite ich einen Überblick über die aktuelle Forschung zu Konflikten im Kontext von land grabbing. Dabei beziehe ich mich vor allem auf Arbeiten aus der Politischen Ökologie als einem relevanten Forschungsfeld für die Analyse von Konflikten um den Zugang zu, die Kontrolle über und Rechte an Land. Insbesondere arbeite ich in diesem Abschnitt zentrale Erkenntnisse hinsichtlich der Gegenstände, Akteure und typischen Akteurskonstellationen in Konflikten im Kontext von land grabbing heraus und stelle aktuelle konzeptionelle Ansätze für die Analyse von Konflikten dar. Im zweiten Abschnitt fasse ich die wesentlichen Debatten zur Bedeutung sozialer Zugehörigkeit für den Zugang zu und die Kontrolle über Land in traditionellen Landverwaltungssystemen zusammen. Diesbezüglich zeige ich, inwiefern die koloniale Interpretation von »Tradition« die gegenwärtige Ausgestaltung traditioneller Landverwaltungssysteme bestimmt (hat). Ein zentrales Interesse gilt in diesem Abschnitt zudem der Wiederbelebung neotraditioneller Landrechtsansprüche in den letzten Jahrzehnten, dem Wechselverhältnis von Macht und Kontrolle über Land sowie dem Zusammenhang von sozialer Zugehörigkeit, Zugang zu Land und Konflikten. Im dritten Abschnitt erarbeite ich zentrale Erkenntnisse aus der Forschung zu traditionellen Autoritäten als Intermediäre. Ich gehe insbesondere 
auf zahlreiche Studien aus der historischen Forschung ein, die analysieren, inwiefern es sich bei der vermittelnden Rolle traditioneller Autoritäten um eine Zuschreibung aus der Kolonialzeit handelt. Darüber hinaus erläutere ich, in welchem Kontext traditionelle Autoritäten in jüngerer Zeit erneut als Intermediäre entdeckt wurden, welche Argumente diese Entwicklung begünstigten und welche Kritikpunkte diesbezüglich existieren.

\subsection{Land grabbing und Konflikte}

Land grabbing ist weder ein neues Phänomen (Alden Wily 2012), noch ein neuer Begriff (Barnes 1971). Dennoch hat das Thema in den letzten Jahren eine neue Prominenz erlangt. Spätestens die Veröffentlichung des GRAIN-Reports "Seized! The 2008 land grab for food and financial security « (GRAIN 2008), machte den Begriff land grabbing erneut berühmt und brachte dem Thema eine breite mediale und wissenschaftliche Aufmerksamkeit ein. Seither forschen Wissenschaftler*innen unterschiedlicher Fachdisziplinen und Forschungsfelder dazu.

Zahlreiche Beiträge betonen, dass land grabbing oftmals mit Konflikten einhergeht (vgl. u.a. Borras/Franco 2012; Hall et al. 2015; White et al. 2012; Wolford et al. 2013). Beiträge, die diese Konflikte erforschen liegen insbesondere in Form von Einzelfallstudien vor (Crawford/Botchwey 2017; Dietz 2019; Engels 2021a; Ndi/Batterbury 2017; Prause/Le Billon 2020). Einen wichtigen Beitrag zur systematischen Analyse von Konflikte, die in Verbindung mit land grabbing entstanden, haben Arbeiten aus der Politischen Ökologie geleistet. Mit ihrem Fokus auf die Untersuchung von Konflikten um die Verteilung natürlicher Ressourcen (Martinez-Alier 2002) hat die Politische Ökologie auch den aktuellen Stand der Forschung zu land grabbing und Konflikten maßgeblich bereichert.

\section{Forschung zu Konflikten um land grabbing und der Beitrag der Politischen Ökologie}

Die Politische Ökologie ist ein interdisziplinäres Forschungsfeld, das sich an einem marxistisch inspirierten Verständnis des Natur-Gesellschaftsverhältnisses orientiert (Watts/Peet 2004). Theoretisch beziehen sich Arbeiten aus diesem Feld vor allem auf kritische neo-marxistische, feministische, post-strukturalistische und post-koloniale Ansätze (Dietz/Engels 2017). Arbeiten aus der 
Politischen Ökologie betrachten Gesellschaft und Natur nicht als voneinander getrennt, sondern als wechselseitig konstitutive Sphären (Edelman et al. 2013; White et al. 2012; Wolford et al. 2013). Sie betonen den inhärent politischen Charakter von Konflikten um Natur und rücken in diesem Zusammenhang zugrundeliegende Macht- und Herrschaftsstrukturen in den Fokus der Analyse (Robbins 2012; Watts/Peet 2004). Aus Sicht der Politischen Ökologie sind Konflikte um Land und Rohstoffe durch Macht- und Herrschaftsverhältnisse strukturiert. Macht- und Herrschaftsverhältnisse sind ihrerseits maßgeblich dadurch konstituiert sind, wer Kontrolle über und Zugang zu Land und Rohstoffen hat (Wissen 2015). Damit bilden diese Arbeiten einen Gegensatz $\mathrm{zu}$ frühen Medienberichten, aktivistischen und wissenschaftlichen Beiträgen zu land grabbing und Konflikten, die weitgehend linear argumentierten (Dietz/Engels 2020). Eine typische Argumentationslinie ist, die großflächige Umnutzung von Land führe zur Vertreibung vorheriger Landnutzer*innen oftmals als homogene lokale Gemeinschaft (local community) gefasst - die daraufhin mit Widerstand reagierten, wodurch ein manifester Konflikt entstünde (kritisch dazu Borras/Franco 2013; Hall et al. 2015). Arbeiten aus der Politischen Ökologie brechen mit dieser linearen Logik und beziehen sich auf kontextabhängige, multi-skalare und relationale Erklärungsansätze. In der Analyse von Konflikten, die im Zusammenhang mit land grabbing stehen, berücksichtigen Arbeiten aus der Politischen Ökologie sowohl globale Transformationsprozesse und die damit einhergehenden sozial-räumlichen, ökologischen und strukturellen Veränderungen als auch das Handeln der involvierten Akteure (Borras/Franco 2013; Dietz/Engels 2020; Hall et al. 2015). In Bezug auf Letztere verweisen aktuelle Beiträge vermehrt darauf, dass vermeintlich homogene Gruppen (beispielsweise Landnutzer*innen) differenziert zu betrachten und Kategorien wie Klasse, Geschlecht, Alter, race, ethnische und politische Zugehörigkeit zu berücksichtigen seien (Escobar 2006; Hall et al. 2015; Kirst/Prause 2019; Porro 2010). Beiträge aus dem Feld der Politischen Ökologie betonen darüber hinaus den inhärent politischen Charakter der Aneignung, Verteilung und des Verbrauchs natürlicher Ressourcen. Diese Prozesse seien eng mit gesellschaftlichen Eigentums- und Kontrollverhältnissen sowie zugrundeliegenden Machtstrukturen verknüpft (Bryant/Bailey 1997).

Aus der Perspektive der Politischen Ökologie sind Konflikte in ihrem jeweiligen historischen Kontext verwurzelt, finden in sozialen Beziehungen Ausdruck, stehen aber auch in Zusammenhang mit übergreifenden globalen Transformationsprozessen und Machtverhältnissen (Peluso/Watts 2001: 5). 
Konflikte werden aus dieser Perspektive nicht als zwingend negativ oder zerstörerisch gewertet, sondern als Möglichkeit, gesellschaftlichen Wandel zu begünstigen (Le Billon 2015: 599). Um Potenziale von Konflikten im Kontext von land grabbing für gesellschaftlichen Wandel erkennen zu können, sei ein tieferes Verständnis der sozialen, ökologischen und politischen Vermittlung dieser Konflikte nötig. In Bezug auf die politische Vermittlung werfen aktuelle Forschungen zunehmend die Frage nach der Rolle des Staates im Zusammenhang mit land grabbing auf (Boone 2015; Engels/Dietz 2017; Lavers 2012; Robbins 2008). Insbesondere fordern sie die Darstellung früherer Beiträge heraus, die Staaten entweder als »Zielländer« oder »Gastgeberländer« (host countries) von land grabbing einstufte. Als »Zielländer « gelten Staaten, die zu schwach sind, um ihre Grenzen und ihr Territorium vor ausländischen Investoren $\mathrm{zu}$ »schützen«. Als »Gastgeberländer« gelten solche, die mittels staatlicher Unternehmen oder durch die Bereitstellung von Infrastruktur und Informationen land grabbing ermöglichen oder unterstützen (Hall et al. 2015). Aktuelle Analysen hinterfragen diese Sichtweise und sprechen sich gegen eine Konzeptualisierung des Staats als homogenen Akteur aus, der mit einer Stimme spreche und einer einheitlichen Handlungslogik folge. Sie fassen den Staat nicht als kohärente Einheit, sondern analysieren stattdessen das Zusammenspiel unterschiedlicher, in Konkurrenz um politischen Einfluss zueinander stehender Institutionen, Akteuren, Interessen und Gruppierungen. Denn staatliche Akteure seien je nach Kontext und Zeitpunkt immer auch zivilgesellschaftliche Akteure. Daraus leite sich die Relevanz ab, die Rolle des Staates immer in Bezug zum jeweiligen Kontext zu sehen. Denn ebenso wie vielfältige Sichtweisen und Interessen innerhalb des Staates existierten, gebe es auch vielfältige Beziehungen zwischen Staat und Gesellschaft (Hall et al. 2015: 477; Schenk 2018).

\section{Gegenstände, Akteure und Akteurskonstellationen in Konflikten um land grabbing}

Die bisherige Forschung verdeutlicht, dass in Konflikten, die im Zusammenhang mit land grabbing auftreten zahlreiche unterschiedliche Themen im Mittelpunkt stehen. Arturo Escobar (2006) schlägt eine Analyse der Konflikte anhand von drei zentralen zusammenhängenden Bereichen vor: Ökonomie, Ökologie und Kultur. Die Relevanz dieser drei Bereiche erklärt er über einen »dreifachen Wandel« (triple transformation), den die Kommodifizierung natürlicher Ressourcen nach sich zöge (Escobar 2006: 7). Mit dem Termi- 
nus des »dreifachen Wandels« beschreibt Escobar den Wandel einer diversifizierten lokalen, teilweise subsistenzorientierten und auf Eigenproduktion ausgerichteten Wirtschaft hin zu einer monetarisierten, marktorientierten Wirtschaft; den Wandel komplexer Ökosysteme in "moderne Formen von Natur« wie Plantagen und Monokulturen; und den Wandel ortsbezogener, lokaler Kulturen hin zu solchen, die vermehrt dominanten Kulturen des globalen Nordens gleichen (müssen). Aus dem dreifachen Wandel ließen sich unterschiedliche Konfliktthemen ableiten, die letztendlich alle mit Fragen gesellschaftlicher Verteilung zusammenhingen: Verteilung von Einkommen und Gewinn, von natürlichen Ressourcen und von sozialer Macht. Letztere bestimme maßgeblich den Handlungsspielraum der Akteure und habe einen Einfluss darauf, wie Mitbestimmung ausgestaltet ist und inwiefern marginalisierte Gruppen oder Minderheiten durch herrschende Akteure anerkannt, berücksichtigt und integriert werden (Escobar 2006).

Andere Arbeiten fassen Konflikte im Zusammenhang mit land grabbing als Konflikte um den Zugang zu und die Kontrolle über Land (Peluso/Lund 2011). Zugang beschreibt in diesem Kontext nicht den physischen Zugriff auf Land, sondern die Fähigkeit einen Nutzen daraus ziehen zu können (Ribot/Peluso 2003). Diese Fähigkeit hängt maßgeblich mit bestehenden Landrechten zusammen, die Besitzverhältnisse gestalten und bestimmte Akteure ermächtigen, Kontrolle über den Zugang zu Land auszuüben. Je nach Kontext können dies traditionelle Autoritäten, der Staat oder Individuen sein (Boone 2013, 2015). De jure Besitzverhältnisse allein - seien sie aus mündlich übermittelten Gewohnheitsrechten oder schriftlich festgehaltenen formalisierten Rechten abgeleitet - spiegeln den vielschichtigen Zugang zu Ressourcen jedoch in der Regel nicht ausreichend wider. Im Fokus der Analyse stehen in der Politischen Ökologie daher die sozialen Beziehungen. Im Zusammenspiel mit strukturellen Faktoren bestimmen sie, wer die Fähigkeit besitzt, Kontrolle über den Zugang zu Land auszuüben, und wer Zugang zu Land nur indirekt erhält. Jesse Ribot und Nancy Peluso (2003) arbeiten strukturelle und relationale Mechanismen heraus, die zentral für den Zugang zu Land seien. Diese Mechanismen sind Zugang zu Technologie, Kapital, Arbeit, Märkten, Wissen, Behörden oder traditionellen Landrechtsinstitutionen und den $\mathrm{Zu}$ gang zu Land über soziale Zugehörigkeit. Konflikte, die im Zusammenhang mit land grabbing auftreten, sind aus dieser Perspektive Ausdruck der Aushandlung unterschiedlicher Formen von Zugang zu Land. Sie manifestieren sich in unterschiedlichen Formen des Widerstands gegen Enteignung und Vertreibung (Borras/Franco 2013; Engels/Dietz 2011), in Protesten gegen Aus- 
beutung, in Forderungen nach Integration und Teilhabe in Form von Arbeitsplätzen (Edelman et al. 2013; Li 2011; Oya 2013) sowie nach Partizipation und Mitbestimmung (Borras/Franco 2013) und in Widerstand gegen die Zerstörung von livelihoods (Escobar 2006). Neben Fragen der Ausgestaltung und Umsetzung agrarindustrieller Projekte werden in Konflikten im Zusammenhang mit land grabbing oftmals auch gegensätzliche Weltanschauungen und Naturvorstellungen sowie übergeordnete Forderungen nach gesellschaftlichem Wandel, sozialer Teilhabe und kultureller Anerkennung verhandelt. Diese betreffen häufig die Ausgestaltung von Landrechten, aber auch die Herausforderung dominanter Entwicklungsmodelle oder neoliberaler Wirtschaftspolitik, z.B. über die Verteidigung kleinbäuerlicher Landwirtschaft gegenüber der Ausweitung einer globalisierten agrarindustriellen Produktion (Dietz/Engels 2017).

Ebenso wie die zentralen Konfliktthemen unterscheiden sich Konflikte im Zusammenhang mit land grabbing auch hinsichtlich der beteiligten Akteure und der auftretenden Akteurskonstellationen. Anhand einer Auswahl an Studien bislang erforschter Konflikte, die im Zusammenhang mit land grabbing auftraten, schlagen Saturnino Borras und Jennifer Franco (2013) eine erste Typologie der involvierten Konfliktakteure und häufig auftretenden Akteurskonstellationen vor. Sie identifizieren drei Obergruppen von Konfliktakteuren, die typisch für Konflikte im Zusammenhang mit land grabbing sind: poor people, Unternehmen und staatliche Akteure. Traditionelle Autoritäten berücksichtigen sie nicht als Konfliktakteure.

Mit der Bezeichnung poor people knüpfen sie an den von Joan MartínezAlier (2002) geprägten Ausdruck environmentalism of the poor an. Dieser bezieht sich auf Konflikte, in denen marginalisierte indigene oder ethnische Gemeinschaften ihren Zugang zu natürlichen Ressourcen verteidigen. In der Regel forderten sie in diesen Konflikten kapitalistische Wirtschaftsmodelle heraus, die in einem Gegensatz zu den Lebensrealitäten vor Ort stünden (Escobar 1996; 2006: 7). Die meisten Studien zu land grabbing und Konflikten widmen sich in ihrer Analyse ebenfalls marginalisierten Gruppen von Landnutzer*innen in Ländern des Globalen Südens, die in den meisten Fällen ihr Land an ausländische Investoren verlieren. Andere Studien zeigen, dass das Phänomen nicht auf den Globalen Süden beschränkt ist und auch die Investoren differenziert betrachtet werden müssen (Brunner 2019; Franco/Borras 2013; van der Ploeg et al. 2015). Darüber hinaus weisen Borras und Franco (2013), wie auch viele andere aktuelle Beiträge (u.a. Boamah 2014a: 407; Gilfoy 2014; Hall et al. 2015; Ubink 2008a: 127), auf die Relevanz der sozialen Differenzie- 
rung der von land grabbing betroffenen Menschen hin. Mit der Verwendung der Bezeichnung poor people suggerieren sie jedoch gleichzeitig eine gewisse Homogenität der Gruppe, die gleichermaßen Kleinbäuerinnen und -bauern, Vertragslandwirt*innen, lokale Eliten, Kleinunternehmer*innen, traditionelle Autoritäten etc. umfassen kann. Unter Berücksichtigung dessen und in Abgrenzung dazu verwende ich in meiner Arbeit anstelle von poor people den Begriff Landnutzer*innen.

Laut Borras und Franco (2013: 1726) treten Konflikte im Zusammenhang mit land grabbing in den folgenden drei Akteurskonstellationen auf: zwischen Landnutzer*innen und Unternehmensakteuren, zwischen Landnutzer*innen und staatlichen Akteuren sowie zwischen unterschiedlichen Landnutzer*innengruppen. Der Fokus ihrer Analyse liegt auf den von land grabbing betroffenen Menschen und ihren jeweiligen Reaktionen. Dementsprechend vernachlässigen sie Konflikte zwischen Unternehmensakteuren und staatlichen Akteuren sowie zwischen unterschiedlichen Behörden. Insbesondere Letztere finden bislang in der Forschung zu land grabbing und Konflikten noch unzureichend Beachtung (Schenk 2018), ebenso wie Konflikte zwischen traditionellen Autoritäten und Landnutzer*innen, die im Zusammenhang mit land grabbing auftreten (Ausnahmen sind Campion/Acheampong 2014; Capps/Mnwana 2015; Mnwana 2019). Den von Borras und Fraco aufgeführten Akteurskonstellationen lassen sich ihrer Analyse entsprechend typische Konfliktgegenstände zuordnen.

Gegenstände von Konflikten zwischen Landnutzer*innen und Unternehmensakteuren seien hauptsächlich solche, die an anderer Stelle als Konflikte um Ausbeutung gefasst würden (vgl. hierzu Edelman et al. 2013). In diesen Konflikten gehe es insbesondere um die Ausgestaltung von Arbeitsbedingungen und die Verbesserung vertraglicher Vereinbarungen. Aber auch die Integration vorheriger Landnutzer*innen durch die Vergabe von Arbeitsplätzen oder im Rahmen von Vertragslandwirtschaft spiele eine Rolle. Zunehmend rückten zudem Umweltthemen wie z.B. die Verschmutzung von Wasser und Boden durch den Gebrauch von Chemikalien und damit zusammenhängende Gesundheitsaspekte in den Fokus (Borras/Franco 2013: 1728).

Konflikte zwischen Landnutzer*innen und staatlichen Akteuren seien vor allem solche, die an anderer Stelle als Konflikte um Enteignung gefasst würden (vgl. hierzu Edelman et al. 2013). Zentrale Themen in diesen Konflikten sind die Vertreibung von Kleinbäuerinnen und -bauern von ihrem Land und damit einhergehenden Umsiedlungsmaßnahmen oder Entschädigungszahlungen. Empirische Beispiele unterstreichen die Erkenntnis, dass der Ver- 
lust von Agrar- und Gemeindeland durch Vertreibung oder Enteignung zentraler Gegenstand in Konflikten im Kontext von land grabbing sei (Campion/Acheampong 2014: 6339; Edelman et al. 2013: 1527; White et al. 2012: 629). In vielen Fällen seien diesbezüglich jedoch nicht der Staat, sondern die Unternehmen für die Umsiedlung zuständig und Konflikte verliefen entsprechend zwischen Unternehmensakteuren und Landnutzer*innen (Drechsel et al. 2019).

In Konflikten zwischen unterschiedlichen Nutzer*innengruppen sind oftmals die unterschiedliche Betroffenheit lokaler Akteure und die damit einhergehenden Haltungen gegenüber land grabbing von Bedeutung. Nicht selten könne innerhalb einer Gemeinschaft beobachtet werden, dass einige Landnutzer*innen sich für und andere gegen ein bestimmtes Projekt aussprechen (Borras/Franco 2013: 1728). Die zentrale Bedeutung sozialer Differenzierung in der Analyse von Konflikten unterstreicht auch Pauline Peters (2004, 2013a, 2013b). In ihren Arbeiten führt sie die vermehrt auftretenden sozialen Konflikte um Land auf einen strukturellen Wandel der Gesellschaft und insbesondere eine zunehmende Klassenbildung zurück. Eine Anzahl an empirische Studien untermauern diese Erkenntnisse. Anhand einer Fallstudie zu einem agrarindustriellen Reisanbauprojekt in Mali zeigt Nicolette Larder (2015), dass betroffene Landnutzer*innen sowohl Allianzen für als auch Allianzen gegen das Projekt eingehen. Larder argumentiert, dass insbesondere Landnutzer*innen, die sich vom Staat im Stich gelassen fühlen und die abgesehen von ihrem Land keine alternative Einkommensquelle haben, agrarindustriellen Projekte gegenüber häufig positiv eingestellt sind. Sie sähen ihre Integration in diese Projekte als einzige Möglichkeit ihren Lebensstandard zu verbessern. Frankline Ndi und Simon Batterbury (2017) unterstreichen mit ihrer Fallstudie zu einem großflächigen Palmölprojekt in Kamerun, dass lokale Landnutzer*innen keinesfalls eine homogene Gruppe bilden. Mit ihrer Forschung zeigen sie, dass benachbarte Gruppen von Landnutzer*innen in Kontexten großflächiger Landumnutzung um die Sicherung des Zugangs zu Land konkurrieren und die Implementierung des agrarindustriellen Projekts daher zu Konflikten um Land zwischen unterschiedlichen Gemeinschaften führe. In diesen Konflikten handelten benachbarte Gemeinden die Ausübung von Kontrolle über zuvor gemeinschaftlich genutztes Land aus, mit der Aussicht es dem Unternehmen gegen Abgaben zur Verfügung stellen zu können. Eine ähnliche Beobachtung machen auch Burnod et al. (2013). Sie argumentieren, dass die Neuverhandlung vormals unklarer oder flexibler Grenzen und Zuständigkeiten in der Verwaltung von Land in Konflikten, die im Zusammenhang mit 
land grabbing auftreten von zentraler Bedeutung sei. In dem von Ndi und Batterbury (2017) untersuchten Fall, seien neben intergemeinschaftlichen Konflikten aber auch solche zwischen Landnutzer*innen und Unternehmen sowie Landnutzer*innen und lokalen Eliten, der nationalen Regierung oder anderen Unterstützer*innen der Unternehmen von Relevanz. Diese mehrfache Form der Verteidigung des Zugangs zu Land - einerseits gegen das Unternehmen und andererseits gegen andere Dörfer - spiegele die Heterogenität und soziale Differenzierung der Landnutzer*innen wider. Für die Ausgestaltung von Konflikten sei insbesondere die Vorherrschaft lokaler Eliten und damit im Umkehrschluss auch der Ausschluss lokaler Gemeinschaften in Verhandlungen großflächiger Landtransaktionen zu berücksichtigen. In dem von ihnen untersuchten Fall handelten lokale Eliten - zu denen sie auch traditionelle Autoritäten zählen - nach Eigeninteressen und nicht, wie es ihre Aufgabe gewesen wäre, im Interesse der Gemeinschaft (Ndi/Batterbury 2017: 47). Zu dieser Erkenntnis kommen auch empirische Studien aus anderen Ländern (Ahmed et al. 2018; Bartels et al. 2018; Nolte 2013). Der daraus resultierende Vertrauensverlust der lokalen Bevölkerung in traditionelle Autoritäten führe laut Ndi und Batterbury (2017) dazu, dass traditionelle Konfliktlösungsansätze seltener angewandt und stattdessen der Weg über das staatliche Rechtssystem in Anspruch genommen würde.

Konflikte zwischen unterschiedlichen Landnutzer*innen entstünden, wie andere Autor*innen betonen, zudem aus einer neuen Konkurrenz zwischen unterschiedlichen Gruppen von Landnutzer*innen. Durch den Wegfall von Gemeindeland, das vormals für vielfältige Zwecke genutzt wurde, seien Nutzer*innen gezwungen, auf andere Flächen auszuweichen und träten dadurch in Konkurrenz zu anderen Nutzer*innen. Ein prominentes Beispiel dafür sind Konflikte zwischen Landwirt"innen und Tierhalter"innen. Erstere nutzen das Land zum Anbau von Nahrungsmitteln, während Letztere es als Weidefläche für ihre Tiere benötigen, womit oftmals Schäden an der Ernte einhergingen (De Schutter 2011: 257).

Im Zusammenhang damit steht die Vergabe von »Ödland« (wastelands) (Cotula/Vermeulen 2009: 1241) - an anderer Stelle auch als »ungenutztes«, »leeres« oder »Grenzland (underutilized, empty, marginal land) bezeichnet (Peters 2013a: 15) - auf die zahlreiche Beiträge kritisch hinweisen. In der Regel handelt es sich dabei um Gemeindeland, für das niemand formalisierte Besitzrechte vorweisen kann und das staatliche Akteure gegenüber Investoren daher oft als ungenutzt bezeichnen. Jedoch ist Land, auf dem agrarindustrielle Projekte umgesetzt werden, fast nie »leer« oder »ungenutzt«. Denn 
soziale Gruppen nutzen Land auf unterschiedliche Weise, wie eine Vielzahl an empirischen Studien belegt (Borras/Franco 2013; Daley 2011; Murphy et al. 2017: 681f.). Diese beschreiben Land als die Grundlage vielfältiger ökonomischer Tätigkeiten wie kleinbäuerlicher Landwirtschaft, Wanderweidewirtschaft, Forstwirtschaft und dem Sammeln von Feuerholz, medizinischen Kräutern, Pflanzen und Früchten.

Vor allem letztere Tätigkeit, die hauptsächlich Frauen ausüben, fände bei der Umsetzung agrarindustrieller Projekte selten Berücksichtigung (Doss et al. 2014). Auch Katja Behrman et al. (2012) machen auf die besondere Betroffenheit von Frauen in diesem Kontext aufmerksam. Konflikte entstünden unter anderem in Zusammenhang mit der Exklusion von Frauen in Konsultierungsverfahren, durch ungleichen Zugang zu und ungleiche Kontrolle über Land von Männern und Frauen oder dadurch, dass geschlechter-spezifische Formen der Landnutzung nicht ausreichend berücksichtigt würden. Ebenfalls konfliktiv seien Szenarien, in denen nur Sektoren gefördert würden, in denen hauptsächlich Frauen arbeiten. Dadurch entstehe ein Ungleichgewicht in der Verteilung von Gewinnen, das zu innergemeinschaftlichen Konflikten führen könne. Auch wenn sich Geschlecht als eine wichtige Analysekategorie in Konflikten, die im Zusammenhang mit land grabbing stehen, erwiesen hat, haben damit zusammenhängende Aspekte in der bisherigen Forschung kaum Aufmerksamkeit erfahren (Borras/Franco 2013). Darüber hinaus sei Land nicht nur die Grundlage für die Ausübung wirtschaftlicher Tätigkeiten, sondern habe in vielen Gemeinschaften auch eine wichtige kulturelle Bedeutung. Es diene unter anderem der Durchführung kultureller und spiritueller Rituale, könne Ruhestätte der Ahnen oder heiliges Land sein (Borras/Franco 2013: 1726f.). Sein Wert gehe weit über den rein ökonomischen hinaus (Escobar 2006).

Die herausgearbeiteten Akteurskonstellationen treten in Konflikten im Kontext von land grabbing selten in einzelner Form auf. Fast immer überlappen sich unterschiedliche Konflikte oder treten parallel auf. So kämpfen in einem Fall Landnutzer*innen ohne formelle Eigentumsrechte vielleicht um Fragen der Teilhabe oder Entschädigung, während Vertragslandwirt*innen um die Ausgestaltung der Arbeitsbedingungen und Landeigentümer*innen mit entsprechenden formellen Eigentumsrechten gegen Enteignung kämpfen. Am häufigsten zu beobachten seien, so Hall et al. (2015: 471), Konflikte zwischen Landnutzer*innen und Unternehmen in Verbindung mit solchen zwischen Landnutzer*innen und dem Staat. 
Neben einer Vielzahl kritischer Beiträge zu Konflikten, die im Zusammenhang mit land grabbing stehen, existieren vermehrt auch solche, die sich mit möglichen Potenzialen großflächiger Landumnutzung befassen. Sie beziehen sich, wie Festus Boamah (2014b: 327) es fasst, auf einen Diskurs des development optimism. Das bedeutet, dass sie die inhärenten Gefahren von land grabbing anerkennen, sie jedoch vor allem auf mangelnde Transparenz und unvorteilhafte vertragliche Regelungen zurückführen. Sie setzen Konflikte, die im Zusammenhang mit land grabbing auftreten nicht in Bezug zu übergeordneten Gegenständen wie Enteignung oder Ausbeutung, sondern betonen hauptsächlich das Problem der negativen Auswirkungen. Sie beziehen sich vor allem auf Höhe und Auszahlungsmodi von Entschädigungszahlungen, Partizipation und Mitbestimmung in der Ausgestaltung der Verhandlungen und Umsetzung agrarindustrieller Projekte sowie das Fehlen, den Inhalt und die Umsetzung von Vereinbarungen zur sozialen Verantwortung von Unternehmen (Corporate Social Responsibility) (Campion/Acheampong 2014: 6339, 6341; Cotula/Berger 2017; Cotula et al. 2014). Darüber hinaus richten zahlreiche Beiträge ihre Aufmerksamkeit in diesem Zusammenhang auf den fehlenden Informationsfluss und die mangelnde Einbindung betroffener Nutzer*innengruppen von Seiten der Unternehmen, des Staats sowie traditioneller Autoritäten (Cotula et al. 2014: 918; Peters 2013a: 16). Diese Studien hinterfragen land grabbing also nicht per se. Zudem vernachlässigen sie weitgehend die Berücksichtigung zugrundeliegender ungleicher Machtverhältnisse und rücken vielmehr das Entwicklungspotenzial agrarindustrieller Projekte in den Vordergrund. Sie argumentieren, dass dieses die negativen Auswirkungen von land grabbing abschwächen könne.

\subsection{Konflikte um Land und die Bedeutung sozialer Zugehörigkeit}

Konflikte um Land treten nicht erst seit dem Anstieg von land grabbing in den frühen 2000er Jahren auf. Vielmehr ist Land seit jeher ein umkämpftes Gut, und Konflikte um dieses Gut seien, so Sara Berry, nicht allein über knapper werdende Ressourcen oder eine zunehmende Kommodifizierung von Land zu erklären (1992). In vielen Fällen seien sie Ausdruck für die Neuverhandlung von Rechten, die den Zugang zu, die Nutzung von und die Kontrolle über Land bestimmen (Kirst/Engels 2014). Zahlreiche Autor*innen verweisen darauf, dass diese Rechte oftmals in einem wechselseitig konstitutiven Verhältnis zu sozialer Zugehörigkeit, Macht und Herrschaft stünden (Berry 2009b; 
Greco 2015; Lund 2011; Lund/Boone 2013; Sikor/Lund 2009a). Die kommerzielle Nutzung von Land könne bestehende Rechte an Land überlagern, ersetze sie in der alltäglichen Praxis der Landnutzer*innen jedoch nicht (Berry 2017: 109). Für die Analyse von Konflikten um Land seien daher Kategorien sozialer Zugehörigkeit von zentraler Bedeutung. Dies gelte insbesondere für Kontexte, in denen Gewohnheitsrecht oder traditionelle Landrechtsinstitutionen den Zugang zu, die Nutzung von und die Kontrolle über Land bestimmen, wie es in den meisten Ländern Subsahara-Afrikas der Fall ist. Denn in traditionellen Landverwaltungssystemen können verschiedene Landnutzer*innen unter Bezug auf mündlich übermittelte Normen und Regeln vielfache, teils parallel existierende Rechte an Land einfordern. Innerhalb der landbesitzenden Gruppe sind, je nach Status und Position, unterschiedliche Personen für die Vergabe dieser Rechte zuständig (Berry 1993). Die Fähigkeit, den Zugang $\mathrm{zu}$ Land $\mathrm{zu}$ kontrollieren oder bestimmte Rechte an Land einzufordern, ist in der Regel eng mit der vermeintlichen Herkunft einer Person oder ihrer Zugehörigkeit zu einer bestimmten (ethnischen) Gruppe verknüpft. Bis heute bestimmen das Erbe der Kolonialpolitik und deren Einfluss auf die Bedingungen, die den Zugang zu Land strukturieren, maßgeblich die Ausgestaltung traditioneller Landverwaltungssysteme (Berry 1992).

\section{Die koloniale Interpretation traditioneller Landrechte}

Wie verschiedene Autor*innen zeigen, war die Ausübung von Kontrolle über Land ein zentrales Anliegen aller Kolonialregime (Amanor 1999; Berry 1992; Peters 2013b). Ziel der Kolonialregime sei es gewesen, anhand der Kontrolle über Land politische Macht über die auf dem Land lebenden Personen zu erlangen und aufrechtzuerhalten sowie gleichzeitig Gewinne aus der kommerziellen Nutzung des Landes zu ziehen. Die Gewinne aus der kommerziellen Nutzung des Landes seien jedoch gleichermaßen ein Anreiz für die Kolonialmacht wie auch für die Kolonialisierten gewesen. Dadurch sei eine neue Konkurrenz um Land und damit zusammenhängende Einkünfte durch cash crops oder Lohnarbeit entstanden (Berry 1992). Da die Kolonialregierung einen direkten Zusammenhang zwischen der Ausübung politischer Macht und territorialer Kontrolle sah, strebten sie die Formalisierung traditioneller Landund Nutzungsrechte an. Diese sollte die Kontrolle des Landzugangs lokaler Gemeinschaften erleichtern.

Um unterschiedliche territoriale Gebiete voneinander abzugrenzen und kontrollierbar zu machen, verzeichneten die Kolonialregime zunächst die 
vermeintlichen territorialen Zuständigkeitsbereiche traditioneller Autoritäten auf Landkarten. Gleichzeitig strebten sie eine enge Zusammenarbeit mit lokalen Eliten und chiefs an. Diese brachten den Kolonialbeamt*innen den Inhalt traditionellen Rechts näher und waren später für die Durchsetzung des formalisierten traditionellen Rechts zuständig. Im System der indirekten Herrschaft übertrugen die Kolonialregierungen loyalen, teilweise neu eingesetzten chiefs die Verwaltung der neu geschaffenen Territorien und stärkten auf diese Weise die Verbindung zwischen politischer Macht und Kontrolle über den Zugang zu Land. chiefs hatten nicht nur das Recht Statute $\mathrm{zu}$ erlassen, mithilfe derer sie über Personen in ihrem Gebiet herrschten, sondern sie führten diese Gesetze auch aus und nahmen darüber hinaus die Rolle von Verwaltungsbeamten ein. Des Weiteren waren sie für die Schlichtung jeglicher Konflikte zuständig. Die Amtsgewalt der chiefs vereinigte also in einer einzigen Person judikative, legislative, exekutive und administrative Macht (Mamdani 1996: 23). Diese war jedoch nicht gänzlich unabhängig vom zentralen Kolonialstaat. Kooperationsunwillige oder widerständige chiefs enthob die Kolonialmacht (teilweise gewaltsam) ihres Amts (von Trotha 1996: 80). In diesem Zuge habe sich durch die Erfindung neuer sozialer Kategorien die Kontrolle über Land zugunsten derjenigen verschoben, die in der Hierarchie des traditionellen Herrschaftssystems weiter oben stehen, wie Pauline Peters (2013a: 4) zeigt: von Familienoberhäuptern, Älteren einer Abstammungslinie und village chiefs, hin zu paramount oder territorial chiefs. Hierarchien seien entweder neu geschaffen oder zumindest verstärkt worden. Dadurch verstärkte sich nicht zuletzt auch die Konkurrenz unterschiedlicher traditioneller Autoritäten - insbesondere um Land.

Unter direkter Herrschaft, wie in französischen Kolonien, übernahm offiziell die Kolonialregierung die Landverwaltung. Dieser Anspruch existierte jedoch meist nur in der Theorie, da die Kapazitäten für die Durchsetzung fehlten (Berry 2017: 108). In der Praxis hingegen bestanden traditionelle Landverwaltungssysteme sowohl unter indirekter als auch unter direkter Herrschaft fort, wobei die koloniale Praxis in beiden Fällen einen Einfluss auf ihre Ausgestaltung ausübte. Wie Richard Kuba und Carola Lentz (2003) für Burkina Faso zeigen, erfand die Kolonialregierung unter direkter Herrschaft zwar keine gänzlich neuen sozialen Kategorien, wie es unter indirekter Herrschaft der Fall war, institutionalisierte jedoch ethnische Kategorien als Legitimationselement für politischen Status und politisierte diese durch ihre Festschreibung in räumlich begrenzte Zuständigkeitsbereiche. Indem die Kolonialregierung durch traditionelle Autoritäten verwaltetes Land als ein durch eth- 
nische Zugehörigkeit bestimmtes Territorium fasste, habe sie eine Hierarchie des Landzugangs auf der Landkarte eingezeichnet. Wie auch Mahmood Mamdani (2001) verdeutlicht, betonte die Definition politischer und territorialer Macht mittels festgeschriebener geographischer Grenzen die Bedeutung von Gruppenzugehörigkeit für die Einforderung von Rechten an Land. Denn über die Grenzziehung definierte die Kolonialregierung auch, wer in diesem Gebiet die »Einheimischen « waren, die entsprechend Rechte an Land geltend machen konnten, und wer die »Fremden« waren, deren Zugang zu Land von der Zustimmung der jeweiligen traditionellen Autorität abhing. In der vorkolonialen Praxis seien soziale Grenzen jedoch tendenziell fließend, durchlässig und - anders als die Kolonialbeamt*innen annahmen - nicht deckungsgleich mit territorialen Grenzen gewesen. Der Verlauf historisch gewachsener territorialer Grenzen, die durch unterschiedliche Personengruppen genutztes oder bewohntes Land unterteilten, sei selten klar definiert gewesen. Darüber hinaus fiel die Fähigkeit, Kontrolle über Land ausüben zu können, nicht immer zusammen mit der Herrschaft über alle Personen, die dieses Land nutzten. Das habe die Einteilung in Einheimische und Fremde problematisch gemacht. Je nachdem in welcher vorherigen Beziehung neue Migrant*innen zu Mitgliedern einer Gemeinschaft standen, seien sie bei ihrer Ankunft nicht zwangsläufig als Fremde eingestuft worden. Gesellschaftliche Positionen konnten zudem über die Zeit neu verhandelt werden, was eine feste Einteilung in die eine oder andere Kategorie erschwert habe (Berry 2002: 643ff.).

Die Formalisierung von Land- und Nutzungsrechten erfolgte über die Kodifizierung derjenigen Regeln und Normen in Kolonialrecht, welche die Kolonialregierung als traditionelles Gewohnheitsrecht anerkannte. Gewohnheit sei jedoch, wie Sara Berry (2002: 643) veranschaulicht, ein sich wandelndes Kaleidoskop von Erzählungen und Interessen gewesen. Erzählungen über die Vergangenheit seien vielfältig und oftmals widersprüchlich gewesen. Lokale Regeln, Normen, Gesetze und Bräuche unterschieden sich maßgeblich in $\mathrm{Ab}$ hängigkeit davon, welche Person mit welchen Eigeninteressen Auskunft darüber gab.

Die unterschiedliche Darstellung traditioneller Landrechte sei nicht zuletzt Ausdruck bestehender Kämpfe und Aushandlungsprozesse um die Kontrolle von Land und damit zusammenhängender Macht gewesen (Berry 1992: 333). Durch die Kodifizierung vermeintlich traditioneller, vor allem aber auch widersprüchlicher Regeln und Normen in koloniales Recht habe die Kolonialregierung ein Landrechtssystem erschaffen, das auf bestehen- 
den Konflikten um Macht und sozialer Zugehörigkeit aufbaute. Indem die Kolonialmächte Zugangsrechte $\mathrm{zu}$ Land in direkten Bezug zu Kategorien sozialer Zugehörigkeit setzten, sei diese zum Gegenstand kontinuierlicher Aushandlungsprozesse geworden, um wirtschaftliche und politische Gelegenheiten möglichst zum eigenen Vorteil nutzen zu können. Das Ergebnis seitens der Landnutzer*innen sei eine andauernde Aushandlung darüber gewesen, welchen Einfluss der Zugang zu Land auf die Zuschreibung sozialer Zugehörigkeit habe und wie im Gegenzug soziale Zugehörigkeit den Zugang zu Land bestimme (Berry 1992: 346). Martin Chanock (1991) argumentiert diesbezüglich, traditionelle Landrechte seien während der Kolonialzeit in einem Dialog zwischen Herrschenden und Beherrschten entstanden. Die Kolonialregierung habe durch die »Erfindung« traditioneller Landrechte die Entstehung von individuellem Landbesitz verhindert. Gleichzeitig stand sie Bestrebungen junger Menschen, Land der alleinigen Kontrolle älterer Männer zu entziehen, ambivalent gegenüber. Das kontinuierliche Streben der Kolonialmächte, die richtige, also für sie vorteilhafteste Version von Gewohnheitsrecht zu etablieren, habe bewusst hegemoniales, in der Praxis jedoch instabiles Wissen produziert. Kolonialmächte erachteten Tradition und Gewohnheit als etwas Zeitloses. chiefs und Dorfälteste, die vorgaben vermeintliche Traditionen $\mathrm{zu}$ kennen, erzählten jedoch widersprüchliche Geschichten. Gewohnheit selbst sei dadurch zu einer scheinbar festen Menge an Grundsätzen geworden, die jedoch durch andauernde geschichtliche Interpretation und Aushandlung gleichzeitig Gegenstand kontinuierlicher Veränderung gewesen seien (Berry 2002: 645). Die indirekte Herrschaft der Kolonialregime, habe es weder ermöglicht ein neues Rechtssystem zu erschaffen, noch das traditionelle zu erhalten oder ihre erfundene Version »traditioneller« afrikanischer Gesetze zu etablieren. Aufgrund ungenügender finanzieller Mittel und Durchsetzungskraft sowie fehlender direkter Kontrolle bestimmten die neuerfundenen »traditionellen « Gesetze die koloniale Gesellschaftsordnung nur in der Theorie. In der Praxis führten sie zu Auseinandersetzungen um die Bedeutung von Land für politische Macht und um die Anerkennung konkurrierender Versionen vermeintlicher Tradition. Dies habe wiederum einen Einfluss auf Kämpfe um Herrschaft und um Zugang zu Ressourcen gehabt. Durch die Anerkennung konkurrierender Erzählungen und damit verbundenen Ansprüchen auf territoriale Kontrolle habe die indirekte Kolonialherrschaft die Instabilität der Bedingungen, die den Zugang zu Land bestimmten, gefördert und lokale Herrschaftsstrukturen fragmentiert. 


\section{Traditionelle Landrechtssysteme im Kontext aktueller Entwicklungen}

Was wir heute als Gewohnheitsrecht bezeichnen, ist also nicht der Fortbestand prä-kolonialer Normen, sondern ein Rechtssystem, das auf der kolonialen Interpretation von »Tradition« beruht (vgl. Ranger 1983). Wie Philippe Lavigne Delville (2000: 99) es fasst, gebe es keine »traditionellen« oder gewohnheitsrechtlichen Landnutzungssysteme an sich, sondern nur Landverwaltungsformen, die auf gewohnheitsrechtlichen Prinzipien beruhen. Unter Berücksichtigung historischer Entwicklungen sprechen zahlreiche Autor*innen daher heute von »neo«-traditionellen Landrechtssystemen oder auch "neo«-traditionellen Autoritäten (vgl. Boone 2015; Knierzinger 2011; Lund/Boone 2013). Insbesondere in Bezug auf die jüngere Vergangenheit besteht in der Literatur Konsens, dass sich traditionelle Landrechtssysteme über die Zeit verändern und mit Maßnahmen wie Dezentralisierungsund Strukturanpassungsprogrammen, der Liberalisierung der Märkte, wirtschaftlichem Wachstum und zunehmender agrarindustrieller Nutzung interagieren (vgl. Juul/Lund 2002b; Lentz 2010; Toulmin/Quan 2000; Ubink 2008b). Berry (2017) veranschaulicht dies in ihrem Beitrag zu Veränderungen in der Landpolitik und den Praktiken der Landvergabe seit den 1990er Jahren, die sie an Beispielen aus Ghana und der Elfenbeinküste illustriert. Sie argumentiert, traditionelle Landrechte würden insbesondere dann neu verhandelt, wenn der Bodenwert ansteige (vgl. auch Amanor 1999). Als Beispiel dafür nennt sie die in den 1990er Jahren verbreiteten Bestrebungen, in Ländern des Globalen Südens die Registrierung individueller Landrechte $\mathrm{zu}$ fördern. Durch diese Strategie versuchten nationale Regierungen in Kooperation mit internationalen Entwicklungsorganisationen wie der Weltbank, Eigentumsrechte zu stärken, um auf diese Weise Investitionen in Land anzuregen. Dadurch schrieben sie Land einen neuen kommerziellen Wert zu. In der Praxis habe das dazu geführt, dass Landnutzer*innen neue Ansprüche auf Land stellten oder alte wiederbelebten, um individuelle Landnutzungsrechte an zuvor gemeinschaftlich geteiltem Land zu erhalten.

Die flexible Ausgestaltung von Landrechten habe es in diesem Zusammenhang erlaubt, neu zu definieren, wer unter welchen Bedingungen und auf welcher Grundlage Zugang zu Land erhält und wer in Konfliktfällen entscheiden kann, wessen Rechte als legitim gelten. Diese viel diskutierte Flexibilität und Verhandelbarkeit, die als typisch für Landzugangs- und Nutzungssysteme in Subsahara-Afrika gelten, stellt Peters (2004) infrage. Sie argumentiert, dass allgegenwärtige Konkurrenz und Konflikte um Land auf 
dem afrikanischen Kontinent die Darstellung traditioneller Landrechtssysteme als offen, verhandelbar und anpassungsfähig in Zweifel zögen. Stattdessen führten sie zu einer zunehmenden Exklusion, gesellschaftlicher Spaltung und Klassenbildung. Anstatt von einer allgemeinen Flexibilität und Verhandelbarkeit auszugehen, sei es nötig zu analysieren, wem traditionelle Landrechtsinstitutionen zugutekämen und für wen sie sich nachteilig auswirkten. Dies ist besonders relevant vor dem Hintergrund, dass es seit den frühen 200oer Jahren im Rahmen von Landreformen verstärkte Bestrebungen gibt, traditionelle oder Gewohnheitsrechte offiziell anzuerkennen und in nationales Recht zu integrieren (Toulmin/Quan 2000). Die Betonung der Verhandelbarkeit und Mehrdeutigkeit traditioneller Landrechtssysteme habe zu einer Zunahme von Studien geführt, die sich auf deren Bedeutung für Handlungsmacht und gesellschaftlichen Gestaltungsspielraum konzentrierten. So geriete aus dem Blickfeld, wer die Gewinnenden und Verlierenden seien. Wichtig sei, präzise zu analysieren, welche sozialen und politischen Verhältnisse Landzugang und -nutzung bestimmen, insbesondere mit Hinblick auf Ungleichheit. Die Analyse müsse, unter Rückbezug auf Kategorien wie Ethnizität, Klasse, Geschlecht und Alter berücksichtigen, wer mit welchen Auswirkungen in der Lage sei Landrechte zu verhandeln. Damit schließt Peters an Kojo Amanor (1999) an. Er argumentiert, die Hervorhebung der flexiblen Verhandelbarkeit traditioneller Landrechtssysteme führe dazu, den Einfluss Einzelner auf Debatten darüber, was Tradition ausmache und welche Regeln den Zugang zu Ressourcen bestimmen sollten, zu überschätzen. Seiner Ansicht nach seien nur die Reichen und Mächtigen, über Investitionen in soziale Netzwerke, in der Lage Letzteres festzulegen. Frauen, jungen Menschen und Migrant"innen seien in der Durchsetzung ihrer Forderungen tendenziell benachteiligt. Mit zunehmender Konkurrenz um Land käme es daher nicht nur vermehrt zu Konflikten zwischen Indigenen und Migrant*innen, sondern auch innerhalb von Familien, in denen häufig junge Menschen um die Beständigkeit ihres Zugangs zu Familienland bangten. Die Kommodifizierung von Land und die in diesem Zusammenhang entstehende Vernetzung nationaler Eliten, Staatsangestellter und internationaler Unternehmen durchdrängen Beziehungen zwischen Generationen, Verwandten und Geschlechtern und trügen zu gesellschaftlichen Verhältnissen bei, die durch soziale Ungleichheit gekennzeichnet seien (Amanor 2001).

Auch wenn es übergeordnete Entwicklungen gebe, die soziale Verhältnisse und Zugang zu Land in einen Zusammenhang setzten, sei die Ausgestaltung von Konflikten um Land und die Macht von Akteuren maßgeblich durch 
den jeweiligen Kontext bestimmt. Das verdeutlicht Berry (2017) beispielhaft anhand eines Vergleichs von Ghana und der Elfenbeinküste. Sie argumentiert, dass es zu berücksichtigen gelte, wie und in welchem Rahmen unterschiedliche aktuelle Machtstrukturen, politischer Wettstreit und verschiedene Formen des Landzugangs und der Landnutzung aufeinanderträfen. Während gegenwärtige Kämpfe um Land zu einem gewissen Grad das Erbe der Kolonialherrschaft widerspiegelten, habe sich die Ausgestaltung wirtschaftlicher Möglichkeiten, politischen Wettbewerbs und sozialer Differenzierung in verschiedenen Ländern individuell entwickelt und verändert. Die Ausgestaltung gegenwärtiger Konflikte um Land sei daher keinesfalls allein auf die gemeinsame koloniale Erfahrung indirekter Herrschaft zurückzuführen, sondern immer in Bezug zu aktuellen Entwicklungen zu sehen (Berry 2017: 115).

\section{Die Bedeutung von Macht für Zugang zu Land}

Bezüglich des Verhältnisses von sozialer Zugehörigkeit und Zugang zu Land in traditionellen Landverwaltungssystem widmen sich einige Autor*innen der Frage, wie sich Macht und Herrschaft sowie der Zugang zu und die Kontrolle über Land noch heute wechselseitig bedingen (Sikor/Lund 2009b). Der Zugang zu und der Besitz von Land, so argumentieren Thomas Sikor und Christian Lund (2009a), stünden in engem Zusammenhang mit Macht und Herrschaft. Denn um Zugang zu oder Besitz von Land zu sichern, bedürfe es einer gesellschaftlich legitimierten Institution, welche die Macht habe, diese Rechte anzuerkennen. Die Legitimität einer solchen Institution und die damit zusammenhängende Macht, resultierten unter anderem aus ihrer Anerkennung. Anerkennung zeige sich darüber, dass Individuen, die nach Zugang $\mathrm{zu}$ Land streben, bestimmten Institutionen die Kompetenz einräumten, für die Vergabe und Anerkennung von Landrechten zuständig zu sein.

Für die Frage, wer sich Land aneignen kann, wer es besitzen oder vergeben kann, seien Aspekte wie die Zugehörigkeit zu einer lokalen Gemeinschaft, Status und Zugehörigkeit relevant. In vielen Kontexten ist Autochthonie ein zentrales Konzept für die Legitimierung von Ansprüchen auf Land. Autochthonie bindet kollektive Identität an ein Territorium. Damit grenze dieses Konzept soziale Gruppen, die für sich beanspruchen »schon immer« oder zumindest »schon lange« an einem bestimmten Ort gelebt zu haben, von solchen $\mathrm{ab}$, die erst »später« hinzugekommen seien, was Konsequenzen für den Zugang zu Land habe (Kirst/Engels 2012). Zusätzlich dazu seien in 
den meisten Fällen soziale Kategorien wie Geschlecht, Alter, Klasse, race, Kaste und Ethnizität für die Einforderung von Landansprüchen relevant (Boone 2014; Lund/Boone 2013). Christian Lund (2011) verdeutlicht, dass soziale Zugehörigkeit und Status nicht automatisch Rechte nach sich ziehen, ihre Einforderung jedoch legitimieren können. Fehlende Zugehörigkeit zu einer lokalen Gemeinschaft könne es einer Person im Gegenzug erschweren, Rechte einzufordern. Einmal erworbene Rechte seien nicht notwendigerweise beständig, sondern in erster Linie vorübergehende Erfolge von Bemühungen, Ansprüche auf Land zu stärken und festzuschreiben. Sie stünden im Wettstreit mit anderen Versuchen, sie bloßzustellen oder zu ersetzen (Moore 1978). Ebenso seien auch Gruppengrenzen nicht endgültig definiert, sondern durchlässig. Das ermögliche es Außenstehenden, die Zugehörigkeit zu einer Gruppe und mit ihr auch Ansprüche auf Land zu erlangen. Außenstehende können Gruppenzugehörigkeit beispielsweise über Heirat und damit verbundene Brautgeschenke, über besondere Loyalität gegenüber oder besonderes Engagement in der Gemeinschaft erwerben (Berry 1989; Kirst/Engels 2012).

In Konflikten um Land werde soziale Zugehörigkeit beispielsweise über die engere Definition von Gruppengrenzen neuverhandelt, wodurch sich der Kreis derjenigen, die Rechte an Land auf legitime Weise einfordern können, verkleinere (Peters 2004). Unterschiedliche Studien beschreiben dies für die Elfenbeinküste (Berry 2009b), Botswana (Peters 1994), Malawi (Kishindo 2004), Äthiopien (Feyissa 2005), Zimbabwe (Moore 2005), Kenia (Moore 1991), Südafrika (Capps/Mnwana 2015) und Ghana (Amanor 1999; Ubink 2008b). Konkurrierende Ansprüche auf Land würden in diesem Zusammenhang häufig über historische Präzedenzfälle gerechtfertigt, die relevant für bestehende Besitz- und Machtverhältnisse sind (Berry 2002). In seinem Beitrag $\mathrm{zu}$ Strategien der Legitimierung und Sicherung von Kontrolle über Land illustriert Lund (2013) diesbezüglich, wie unterschiedliche Erzählungen über die Vergangenheit konkurrierende Landansprüche in traditionellen Landrechtssystemen rechtfertigen. Die Kontrolle über Land sei eng an diskursive Strategien geknüpft. Insbesondere die Rekonstruktion der Vergangenheit sowie unterschiedliche Konzeptualisierungen von Raum - entweder als Besitz oder als Territorium - seien in diesem Zusammenhang zentral.

Erkenntnisse aus den Arbeiten $\mathrm{zu}$ Land, sozialer Zugehörigkeit und Konflikten unterstreichen die Bedeutung sozialer Zugehörigkeit. Für mein Forschungsvorhaben ergibt sich daraus die Notwendigkeit, multiple, parallel existierende oder sich überlappende Rechte an Land in der Analyse von Konflikten um Land zu berücksichtigen, da diese in traditionellen Land- 
rechtssystemen oftmals mit Kategorien sozialer Zugehörigkeit verschränkt sind. Je nach sozialer Zugehörigkeit können Individuen und Gruppen sich unterschiedlicher Mechanismen bedienen, über die sie Zugang zu Land erlangen können. Traditionelle Autoritäten kontrollieren einige dieser Mechanismen, jedoch nicht alle. Im Laufe der Zeit können sich diese Mechanismen verändern, da traditionelle Normen und Rechte nicht beständig, sondern verhandelbar und wandelbar sind, wenn auch nicht beliebig und durch jeden. Für die Auswertung meiner empirischen Daten bedeutet dies, präzise - und insbesondere unter Berücksichtigung sozialer Differenzierung - zu analysieren, welche sozialen und politischen Verhältnisse Zugang $\mathrm{zu}$ Land bestimmen und wer in Konflikten um Zugang die Gewinnenden und Verlierenden sind. Der Rückbezug auf Kategorien sozialer Differenzierung ermöglicht es zu erfassen, wer mit welchen Auswirkungen in der Lage ist, Rechte an Land aufrechtzuhalten oder neu zu verhandeln und wer nicht. Bislang findet die soziale Differenzierung lokaler Gemeinschaften in der Forschung zu Konflikten im Zusammenhang mit land grabbing noch unzureichend Berücksichtigung.

\subsection{Traditionelle Autoritäten als Intermediäre}

Um mich der Rolle traditioneller Autoritäten in Konflikten, die im Zusammenhang mit land grabbing auftreten, zu nähern, knüpfe ich in meiner Forschung an eine dritte Debatte an: diejenige zu traditionellen Autoritäten als Intermediäre.

Bereits während der Kolonialzeit, insbesondere unter indirekter Herrschaft, schrieben Kolonialverwalter*innen traditionellen Autoritäten eine vermittelnde Funktion zwischen der Bevölkerung und dem Kolonialstaat zu. Dies belegt eine umfangreiche Literatur aus der historischen Forschung (Crowder 1964; Eckert 1999, 2007; Eriksen 2001). Mit dem Ende der Kolonialzeit verloren traditionelle Autoritäten zunächst an Prominenz in der Politik. Als Intermediären sind sie erst in jüngerer Zeit wieder in den Fokus der Aufmerksamkeit gerückt. Insbesondere internationale NGOs, Geberund Entwicklungsorganisationen haben ihren Nutzen als Mediator*innen und Entwicklungsagent*innen entdeckt. Aktuelle Studien dazu beziehen sich auf unterschiedliche Kontexte, in denen traditionelle Autoritäten als Intermediäre zwischen verschiedenen Akteuren agieren und teilweise sogar bewusst als solche eingesetzt werden. Kritische Forschungen dazu verweisen 
auf eine mangelnde Berücksichtigung der historischen Entwicklung traditioneller Herrschaftssysteme, insbesondere mit Bezug auf die Kolonialzeit. Die fehlende Berücksichtigung der historischen Entwicklung berge die Gefahr, gesellschaftlich verankerte Ungleichheit zu reproduzieren.

\section{Traditionelle Autoritäten als Mediator*innen und Entwicklungsagent*innen}

Mit dem Ende der Kolonialzeit verloren traditionelle Autoritäten zunächst an Prominenz in der Politik. In den 1990er Jahren kam seitens internationaler Geber- und Entwicklungsorganisationen jedoch ein erneutes Interesse an der Kooperation mit traditionellen Autoritäten auf, das Ann Whitehead und Dzodzi Tsikata als »return to the customary« bezeichnen (2003: 68). Das erneute Interesse an der Zusammenarbeit mit traditionellen Autoritäten bezog sich zunächst auf die Bereiche Entwicklungsförderung, lokale Regierungsführung und Konfliktmanagement (Ubink 2008c). Später rückten traditionelle Autoritäten ebenfalls mit Blick auf die dezentrale Verwaltung natürlicher Ressourcen als günstige und kulturell legitime Akteure in den Fokus (Capps 2018). Das erneute Interesse an der Zusammenarbeit mit traditionellen Autoritäten entstand in einem Kontext der Demokratisierung, die mit zunehmender Kritik internationaler Geber- und Entwicklungsorganisationen an der Leistungsfähigkeit und institutionellen Qualität vieler afrikanischer Staaten einherging. Die neu gegründeten Staaten sahen sich mit dem gleichen Dilemma wie schon die Kolonialregierungen konfrontiert: die Verwaltung riesiger Gebiete unter Verfügung geringer Ressourcen und schwacher staatlicher Institutionen in ländlichen Gegenden bewältigen zu müssen (Baldwin 2016). Organisationen wie die Weltbank sahen einen direkten Zusammenhang zwischen der vermeintlich schlechten wirtschaftliche Entwicklung und der schwachen Leistung lokaler staatlicher Institutionen (Brautigam 1992; Hydén/Bratton 1992; World Bank 1989, 1992, 1994). Die Kooperation mit traditionellen Autoritäten sollte die Regierungsführung auf lokaler Ebene stärken und fehlende staatliche Kapazitäten ausgleichen (vgl. z.B. World Bank 2002). Als innovative Strategie fügte sie sich zudem nahtlos in das Bild einer auf der Partizipation der »lokalen Bevölkerung« beruhenden Entwicklungspolitik ein (OECD/DAC 1996: 9). Bei der Wahl traditioneller Autoritäten als Kooperationspartner*innen stand vor allem deren vermeintliches soziales Kapital im Vordergrund. Als kulturell versierte Repräsentant*innen ihrer Gemeinschaft verkörperten sie sozialen Zusammenhalt 
und gemeinschaftliches Handeln. Dadurch seien sie zu einem Sinnbild für eine erfolgreiche nachhaltige Entwicklung geworden, die aus Sicht vieler Entwicklungsorganisationen nur aus einer authentischen lokalen Kultur heraus entstehen könne (Grischow 2008: 64). Im Kontext zunehmender innerstaatlicher Konflikte und einem erhöhten Bedarf an neuen Konfliktlösungsansätzen, stieg parallel auch das Interesse an traditionellen Autoritäten als Mediator*innen in Konflikten. Befürworter*innen traditioneller Konfliktlösungsansätze betonen diesbezüglich die niedrigschwellige Zugänglichkeit traditioneller Institutionen. Sie argumentieren, dass traditionelle Rechtsprechung günstiger, schneller und leichter verständlich sei als der Weg über das staatliche Rechtssystem, das auf viele einen einschüchternden Effekt habe. Konfliktschlichtung sei auch deshalb ein zentraler Aufgabenbereich traditioneller Autoritäten, da diese auf stabilisierende und vereinende Art mit Konflikten und Streitfällen umgingen (Adjei et al. 2017: 314). Seit Beginn der 200oer Jahre beschäftigen sich zahlreiche Autor*innen mit den Möglichkeiten, Herausforderungen und Grenzen traditioneller Konfliktlösungsansätze sowie der Rolle traditioneller Autoritäten in der Bearbeitung von Konflikten. Neben wissenschaftlichen Studien (u.a. Buckley-Zistel 2005; Moe/Simojoki 2013; Rukuni et al. 2015; Santschi 2014; Tubiana et al. 2012; Zartman 2000) kommen Beiträge vermehrt auch aus der entwicklungs- und friedenspolitischen Praxis (Boege 2006; OECD 2001). Einige Autor*innen argumentieren, traditionelle Autoritäten, welche die Kolonialzeit überdauert hätten, verfügten über eine besondere Legitimität innerhalb ihrer Gemeinschaft. Während der Kolonialzeit seien sie häufig zentrale Figuren im antikolonialen Widerstand und damit Sinnbilder kultureller Identität gewesen. Ihre soziale und politische Legitimität leite sich daher auch heute noch aus prä-kolonialer Zeit ab (Ray/Reddy 2003).

\section{Kritische Perspektiven auf traditionelle Autoritäten als Intermediäre}

Argumentationsweisen wie diese rechtfertigten und stärkten die Funktion traditioneller Autoritäten als so genannte Entwicklungsagent*innen (agents of development) oder Entwicklungsvermittler*innen (development broker) (Grischow 2008) und legitimierten ihre zentrale Rolle in der Schlichtung von Konflikten. Sie blieben jedoch nicht unangefochten. Gleichwohl traditionelle Autoritäten im Zuge einer Welle der Demokratisierung als Repräsentant*innen lokaler Gemeinschaften wiederentdeckt wurden, weisen Kritiker"innen darauf hin, dass traditionelle Herrschaft demokratische Prinzipien gefährden kön- 
ne. Denn traditionelle Autoritäten werden in der Regel ernannt und nicht demokratisch gewählt. Gemeinschaften hätten daher kaum eine Möglichkeit, die Rechenschaftspflicht traditioneller Autoritäten einzufordern, so dass ihr Handeln oftmals intransparent sei. Daraus könne sich eine Gefährdung für Errungenschaften der Demokratie wie Gleichheit, Menschenrechte oder Geschlechtergleichstellung ergeben (Buur/Kyed 2007; Ubink 2008c). In Bezug auf die Nachteile traditioneller Konfliktbearbeitung verweist Volker Boege (2006) beispielsweise darauf, dass traditionelle Herrschaftssysteme immer auf gewisse Weise die "gute alte Ordnung« reproduzierten und anfällig für Missbrauch seien. Das könne sich als nachteilig für die schwachen Menschen der Gemeinschaft erweisen.

Insbesondere aus der politischen Anthropologie existiert eine Anzahl an kritischen Analysen, die sich mit traditionellen Autoritäten als Entwicklungsagent*innen oder Entwicklungsvermittler*innen befasst. Im Fokus der Kritik steht unter anderem der Social Capital Ansatz. Befürworter*innen des Social Capital Ansatzes definieren traditionelle Autoritäten hauptsächlich über ihren praktischen Nutzen und ihre Fähigkeit, sozialen Zusammenhalt und gemeinschaftliches Handeln initiieren zu können. Kritiker*innen argumentieren, diese Perspektive berücksichtige nur unzureichend die historische Entwicklung traditioneller Herrschaft (Grischow 2008; Kleist 2011; Knierzinger 2011). Jeff Grischow (2008) verweist darauf, dass Verfechter*innen traditioneller Herrschaft sich tendenziell stärker auf den Zusammenhalt und die Harmonie innerhalb traditionell verwalteter Gemeinschaften konzentrierten, als auf Stratifizierung und Hierarchien. Dieser Trend in der Social Capital Literatur sei eng mit der Tendenz verflochten, durch einen a priori Fokus auf eine kohärente Gemeinschaft, bestehende Konflikte zu übersehen. Eine stärker historische Perspektive zeige, dass viele als traditionell bezeichnete Gemeinschaften stratifiziert, also durch tief verwurzelte Hierarchien sozialer und politischer Macht geprägt sind. Dies zu ignorieren, berge das Risiko, historisch geschaffene und gesellschaftlich verankerte Ungleichheit zu reproduzieren (Capps 2018). Johannes Knierzinger (2011) unterstreicht diesen Punkt. Er argumentiert, dass traditionelle Herrschaft vor allem ein Instrument der Elitenbildung sei und als solches durch vermeintliche kulturelle Werte abgesichert werde. Insbesondere beruhe es auf kulturellem und sozialem Kapital (vgl. Bourdieu 1986). Zu kulturellem Kapital zählen akademische Abschlüsse, Bildung und Wissen. Soziales Kapital kann über Adelstitel oder die Mitgliedschaft in sozialen Netzwerken erworben werden. Anhand einer Analyse zu Entwicklungsvermittler*innen in Ghana kommt er zu dem Schluss, dass 
die Fluidität und Verhandelbarkeit neotraditioneller Herrschaftssysteme es unterschiedlichen Akteuren erlaube, durch besonderes Engagement im Entwicklungsbereich den relativ neuen und daher auch als neotraditionell bezeichneten Titel des Entwicklungs-chiefs (chiefofdevelopment) zu erlangen. Dieser Titel ermögliche den Zugang zum traditionellen Herrschaftssystem, der immer auch mit gesellschaftlichem Aufstieg verbunden sei. Unter anderem erleichtere er den Kontakt zu Politiker*innen, internationalen Akteuren und erhöhe die Möglichkeiten ins Ausland zu gehen. Als problematisch erachtet Knierzinger, dass trotz des relativ einfachen Zugangs zu neotraditionellen Posten noch keine Entmystifizierung des chieftaincy-Systems stattgefunden habe. Ein Hinweis darauf sei die Beobachtung, dass die Macht von chiefs selten herausgefordert würde (ebd.: 41). Neotraditionelle Strukturen, die von außen nicht als solche erkannt würden, könnten zu einem Missbrauch traditioneller Systeme führen. Auch Nauja Kleist (2011) fordert eine stärkere Berücksichtigung neotraditioneller Entwicklungen in traditionellen Herrschaftssystemen. Anhand ihres Beitrags zu modernen chiefs in Ghana illustriert sie die Wandelbarkeit und Vielschichtigkeit traditioneller Herrschaft sowie die Entstehung neotraditioneller Handlungsmuster. Ebenso wie Knierzinger liegt ihr Fokus auf Immigrant"innen, die nach ihrer Rückkehr aus dem Ausland in Ämter traditioneller chiefs berufen werden. Kleist argumentiert, dass diese einen besonderen Status in der Gemeinschaft genössen, da sie mit ihrer internationalen Erfahrung und ihren Kontakten das Streben nach Entwicklung und Modernisierung verkörperten. Gleichzeitig sei es für sie jedoch auch besonders wichtig, ihre Legitimität in der Gemeinschaft zu festigen, indem sie unter anderem anhand von Kleidung, Bräuchen und Normen vermeintlich traditionelle Werte aufrechterhielten. Die Anerkennung und die damit verbundene Legitimität dieser neotraditionellen chiefs würden in einem Spannungsfeld von Tradition und Moderne verhandelt und inszeniert. Beide Sphären fänden gesellschaftlichen Anklang, weshalb neotraditionelle chiefs auch über die Fähigkeit verfügen müssten, sich in beiden positionieren zu können. In der Forschung zu traditionellen Herrschaftssystemen sei es daher von zentraler Bedeutung, das Handeln traditioneller Autoritäten in Bezug auf beide Sphären zu verstehen und es gleichzeitig als lokal und global zu fassen (ebd. : 647).

Wissenschaftliche Beiträge zu traditionellen Autoritäten als Intermediären lassen darauf schließen, dass internationale Geberorganisationen die vermittelnde Rolle traditioneller Autoritäten fördern, obwohl Wissenschaftler*innen diese kritisch hinterfragen. Kritiker"innen nehmen insbesondere das Verhältnis zwischen traditionellen Autoritäten und ihrer Gemeinschaft 
in den Blick. Sie hinterfragen, inwiefern traditionelle Autoritäten tatsächlich die Interessen ihrer Gemeinschaft repräsentieren und inwiefern Mitglieder der Gemeinschaft das Handeln traditioneller Autoritäten herausfordern (können). Im Zentrum der Kritik an traditioneller Herrschaft steht die mangelnde Rechenschaftspflicht traditioneller Autoritäten gegenüber ihrer Gemeinschaft, die oftmals mit intransparentem Handeln einhergeht. Hinsichtlich der entwicklungspolitischen Zusammenarbeit mit traditionellen Autoritäten als Intermediären zwischen ihrer Gemeinschaft und Dritten verweisen Kritiker*innen darüber hinaus auf die mangelnde Berücksichtigung der historischen Entwicklung traditioneller Herrschaft während der Kolonialzeit. Als Instrument der Elitenbildung berge traditionelle Herrschaft das Risiko zu einer Reproduktion und Perpetuierung historisch gewachsener und gesellschaftlich verankerter Macht- und Ungleichheitsverhältnisse zu führen und erweise sich in diesem Zuge zum Nachteil für schwächere Akteure. Mit meiner Forschung baue ich auf diese Erkenntnisse auf und berücksichtige insbesondere die verschiedenen Rollen traditioneller Autoritäten, die ihnen in unterschiedlichen Kontexten zugeschrieben wurden und seither parallel fortbestehen. In meiner Analyse des Handelns traditioneller Autoritäten in Konflikten um Zugang, die im Kontext von land grabbing auftreten, zeige ich inwiefern eine differenzierte Berücksichtigung dieser unterschiedlichen Rollen von Bedeutung für die Erklärung von Konflikten ist.

\subsection{Bezug zur bestehenden Forschung - Anknüpfungspunkte und Leerstellen}

Ziel des Kapitels war es, den aktuellen Stand der Forschung in Bezug auf traditionelle Autoritäten in Konflikten um Zugang zu Land, die im Zusammenhang mit land grabbing auftreten, aufzuarbeiten, zentrale Leerstellen zu identifizieren und Anknüpfungspunkte für meine eigene Forschung zu bestimmen. Dafür habe ich die bisherige Forschung zu (1) Konflikten im Zusammenhang mit land grabbing, (2) Land, sozialer Zugehörigkeit und Konflikten sowie (3) traditionellen Autoritäten als Intermediäre aufgearbeitet.

Aus den Arbeiten zu Konflikten im Kontext von land grabbing lässt sich ableiten, dass es im Kern dieser Konflikte um unterschiedliche Formen der Aushandlung von Zugang zu Land geht und dass Zugang zu Land wiederum eng mit bestehenden Machtverhältnissen zwischen den Konfliktakteuren verknüpft ist. Bislang konzentrieren sich Beiträge zur Analyse dieser Konflik- 
te auf drei Gruppen von Konfliktakteuren: Unternehmensakteuren, staatliche Akteure und die Landnutzer*innen. Die Rolle traditioneller Autoritäten in diesen Konflikten findet hingegen unzureichende Berücksichtigung. Dieser blinde Fleck in der Forschung zu Konflikten, die im Zusammenhang mit land grabbing auftreten, überrascht, da traditionellen Autoritäten in diesen Konflikten eine paradoxe Position einnehmen. Sie ermöglichen den Zugang $\mathrm{zu}$ Land für agrarindustrielle Vorhaben und sind gleichzeitig Vertreter*innen der Interessen ihrer Gemeinschaft. Zentrales Interesse vieler Mitglieder der Gemeinschaft ist jedoch der Zugang zu Land. Dieses Interesse steht im Widerspruch zur Implementierung agrarindustrieller Vorhaben, wie in Konflikten, die in diesem Kontext auftreten, deutlich wird. Über die Analyse des Handelns traditioneller Autoritäten im Rahmen großflächiger Landvergaben und damit einhergehender Konflikte möchte ich diese widersprüchliche Rolle traditioneller Autoritäten in den Blick nehmen und herausarbeiten, inwiefern sie für die Erklärung von Konflikten um Zugang in traditionellen Landrechtssystemen von Bedeutung ist. In meiner Analyse berücksichtige ich insbesondere die soziale Differenzierung innerhalb der Gemeinschaft, die sich über ungleiche Möglichkeiten des Zugangs zu Land ausdrückt. Mit meiner Forschung möchte ich zudem an die Debatte um die Ausgestaltung von Landrechten in traditionellen Landverwaltungssystemen anknüpfen.

Im Rahmen von Landreformen gibt es seit Beginn der $2000 e r$ Jahre eine Tendenz zur Formalisierung traditioneller Landrechte und der Integration von Gewohnheitsrecht in nationales Recht, um Zugang zu Land abzusichern (Chimhowu/Woodhouse 2006:347). Die Debatte darum, wie diese Integration aussehen soll, ist durch eine Dichotomie zwischen der Registrierung individueller Rechte und der Anerkennung kollektiver Rechte. Verfechter*innen der Formalisierung von Rechten beziehen sich insbesondere auf eine Studie von de Soto (2000), der die Mehrdeutigkeit und Verhandelbarkeit traditioneller Rechte als Ursachen für eine geringe Produktivität identifiziert. Die Registrierung von Landrechten war außerdem zentraler Bestandteil marktorientierter Agrarreformen, um den Zugang zu Land für Investoren zu erleichtern (Lahiff et al. 2007). Kritiker*innen dieser Bestrebungen argumentieren, die Registrierung von Landrechten wirke sich nachteilig auf diejenigen Akteure mit ohnehin schwachen Rechten an Land aus und blende sekundäre Rechte, wie die Weidenutzung und das Sammeln von Feuerholz oder wild vorkommender Nahrungsmittel aus (Toulmin 2009; Toulmin/Quan 2000). Folglich sprechen sie sich für die Anerkennung kollektiver Rechte aus. Dafür bedarf es jedoch der Berücksichtigung sozialer Differenzierung innerhalb lokaler Ge- 
meinschaften (Kirst/Prause 2019), die bislang in der Debatte um die Anerkennung kollektiver Rechte noch fehlt, jedoch relevant für die Absicherung des Zugangs zu Land derjenigen mit den schwächsten Rechten ist. Damit die Anerkennung traditioneller Rechte nicht zur Reproduktion sozialer Ungleichheit führt, bedarf es einer differenzierten Analyse von Machtverhältnissen, die in Konflikten um Zugang zu Land im Zusammenhang mit land grabbing besonders deutlich werden. Eine Analyse dieser Machtverhältnisse kann, neben einem besseren Verständnis von Konflikten um Zugang, dazu beitragen ein besseres Verständnis der Herausforderungen traditioneller Land- und Ressourcenverwaltung zu entwickeln und mögliche Potenziale, die sie mit sich bringen kann, zu erkennen.

Im folgenden Kapitel entwickle ich einen Analyserahmen, der es mir ermöglicht, den Zusammenhang zwischen dem Handeln traditioneller Autoritäten im Kontext von land grabbing, den damit einhergehenden Veränderung des Zugangs zu Land und den Konflikten, die in diesem Zusammenhang auftreten, zu untersuchen. 



\section{Zugang zu Land und Macht - Ein Analyserahmen für Konflikte im Kontext von land grabbing}

Um analysieren zu können, welcher Zusammenhang zwischen dem Handeln traditioneller Autoritäten im Kontext von land grabbing und Konflikten um Zugang zu Land besteht, kombiniere ich ein theoretisch begründetes Verständnis von Zugang zu Land in traditionellen Landverwaltungssystemen mit einem Machtkonzept, das auf die empirische Analyse ausgerichtet ist. Das Verständnis der Mechanismen, über die unterschiedliche Akteure Zugang zu Land in traditionellen Landverwaltungssystemen erlangen, strukturiert meine Analyse. Es dient dazu, die unterschiedlichen Interessen der Akteure zu erfassen, entlang derer Konflikte um Zugang zu Land verlaufen. Für die Analyse fehlt jedoch ein erklärendes Element, das erlaubt zu untersuchen, wie sich das Handeln von Konfliktakteuren - und insbesondere das Handeln traditioneller Autoritäten - auf den Zugang zu Land auswirkt, und woher sich die Fähigkeit, Zugang zu Land zu kontrollieren, ableitet. Diesem Zweck dient das empirisch nutzbare Konzept von Macht.

Zugang verstehe ich in Anlehnung an Jesse C. Ribot und Nancy Lee Peluso (2003) als die Fähigkeit, einen Nutzen aus etwas ziehen zu können. Zugang $\mathrm{zu}$ Land bedeutet folglich, einen Nutzen aus dem Land ziehen zu können. Beispiele dafür sind die Bestellung des Lands für den Eigenbedarf oder für den Verkauf, die Verpachtung des Lands gegen Geld- oder Naturalleistungen, Lohnarbeit auf dem Land oder die Weiterverarbeitung von auf dem Land befindlichen Ressourcen. Einen Nutzen aus dem Land zu ziehen bedeutet also, über das Land etwas zu gewinnen, das weiterverwertet werden kann. Im Vergleich zu klassischen Debatten um Eigentum - definiert als »das Recht einen Nutzen aus etwas ziehen zu können« (Ribot/Peluso 2003: 154, Übersetzung durch die Autorin) - ermöglicht das theoretische Konzept des Zugangs eine 
umfassendere Analyse. Statt nur auf Eigentums- und Besitzverhältnisse, lenkt es den Blick auf ein breiteres Spektrum sozialer Verhältnisse, die es Menschen ermöglichen oder sie daran hindern, einen Nutzen aus Land zu ziehen. Damit eignet es sich insbesondere für Analysen im Kontext traditioneller Landverwaltungssysteme. In diesen ist Zugang zu Land nicht primär durch staatliches Recht, das formalisiertes Eigentum schafft, bestimmt, sondern durch eine Vielzahl unterschiedlicher Zugangsmechanismen (vgl. Boone 2015). Um Zugang zu Land analytisch greifbar zu machen, arbeite ich, in Anlehnung an Ribot und Peluso (2003) sowie aufbauend auf Literatur zu traditionellen Landrechtssystemen (Amanor 1999; Berry 1989, 1993; Juul/Lund 2002a; Lund 2011, 2013; Peters 1984, 2004; Shipton/Goheen 1992; Sikor/Lund 2009a) zentrale Mechanismen heraus, die bestimmen wer in traditionellen Landverwaltungssystemen wie und wann in der Lage ist, einen Nutzen aus Land zu ziehen (Ribot/Peluso 2003: 154, Hervorhebung im Original). Unter Berücksichtigung der Literatur zu traditionellen Landrechtssystemen leite ich aus dem Beitrag von Ribot und Peluso zwei Varianten von Mechanismen ab, die als relevant für den Zugang zu Land in traditionellen Landverwaltungssystemen gelten: rechtebasierte sowie relationale und strukturelle Mechanismen.

Studien zu Konflikten um Land im Kontext von land grabbing zeigen, dass Zugang zu Land ein relevanter Konfliktgegenstand ist, der oftmals im Zentrum der Konflikte steht (Peluso/Lund 2011; White et al. 2012). In Konflikten oder Kontexten struktureller Veränderungen können nicht alle Akteure in gleichem Maße zuvor bestehenden Zugang zu Land aufrechterhalten, da sie Zugang zu Land nicht gleichberechtigt kontrollieren. Aus diesem Gedanken leite ich die Annahme ab, dass Kontrolle ein zentraler Aspekt für die Analyse von Konflikten um Zugang zu Land ist. In traditionellen Landverwaltungssystemen obliegt die Kontrolle des Zugangs insbesondere - aber nicht ausschließlich - traditionellen Institutionen. Im Fall von chieftaincy-Systemen fordern je nach Kontext unterschiedliche traditionelle Autoritäten das Recht Zugang zu Land zu kontrollieren für sich ein. Um zu verstehen, welcher $\mathrm{Zu}$ sammenhang zwischen dem Handeln traditioneller Autoritäten und Konflikten um Land besteht, ist es daher wichtig zu analysieren, wie traditionelle Autoritäten Zugang zu Land kontrollieren. Haripriya Rangan bezeichnet Kontrolle als die Fähigkeit, das Handeln anderer zu überwachen, zu lenken oder zu regulieren (1997: 72). Entsprechend üben diejenigen Akteure Kontrolle über den Zugang zu Land aus, die in der Lage sind, zu überwachen, zu lenken und zu regulieren, wer einen Nutzen aus Land ziehen kann. Die Fähigkeit den Zugang zu Land zu kontrollieren, wird wiederum als Ausdruck 
von Macht verstanden (Bryant/Bailey 1997; Ribot/Peluso 2003). Obwohl zahlreiche Beiträge zu Konflikten um Zugang zu Land die analytische Relevanz von Macht betonen (vgl. Bavinck et al. 2014; Peluso/Lund 2011; Ribot/Peluso 2003; Sikor/Lund 2009a), bleiben sie bezüglich folgender drei Fragen vage: (1) Welche Formen kann Macht in Konflikten um Zugang zu Land annehmen? (2) Woher leitet sie sich ab? Und (3) wie können Machtverhältnisse in diesem Kontext analytisch in den Blick genommen werden? Diesen Schwachpunkten widme ich mich in meinem Analyserahmen, indem ich ein differenziertes Verständnis von Zugang zu Land in traditionellen Landrechtssystemen mit einem empirisch nutzbaren Konzept von Macht verknüpfe. Macht verstehe ich als relational, was bedeutet, dass Macht nicht absolut ist, sondern in Verhältnissen zwischen Akteuren besteht und diese gestaltet. Als einen für die empirische Analyse nutzbaren Ansatz zur Untersuchung von Machtverhältnissen integriere ich Steven Lukes' Konzept der drei Dimensionen von Macht in meinen Analyserahmen (Lukes 2005). Mit Blick auf die empirische Analyse bietet es gegenüber anderen Machtkonzepten einige entscheidende Vorteile. Lukes greift zentrale Uneinigkeiten und Widersprüche bezüglich der Konzeption von Macht - die in unterschiedlichen theoretischen Perspektiven auf das Thema zum Ausdruck kommen - auf. Er diskutiert die jeweiligen ontologischen und epistemologischen Standpunkte, die einen Einfluss auf die Entwicklung der unterschiedlichen Machtkonzepte haben, widmet sich ihren Stärken, Schwächen sowie verbindenden Elementen und geht der zentralen Frage nach, wie sie in der empirischen Forschung genutzt werden können. Er betrachtet unterschiedliche Konzeptionen von Macht als sich ergänzend nicht als exklusiv oder einzelne als falsch. Lukes bezieht sich auf Machtforscher*innen wie Max Weber, Robert Dahl, Nelson W. Polsby, Peter Bachrach oder Morton Baratz. Deren Ansätze entwickelt er weiter und integriert sie in ein mehrdimensionales Machtkonzept. Lukes eigener Schwerpunkt liegt auf der Konzeptualisierung der am wenigsten sichtbaren Formen der Macht: Über diese werde Macht am effektivsten ausgeübt (Lukes 2005: 1). Sein wesentlicher Anspruch an das Konzept ist, dass es Offenheit gegenüber empirischen Phänomenen gewährleistet, ohne sich auf eine a priori gesetzte moralische oder politische Perspektive zu beschränken (ebd.: 30). Damit eignet es sich insbesondere für explorativ angelegte Forschungsdesigns - wie dasjenige meiner Studie - die darauf abzielen, neue Erkenntnisse zu einem bisher wenig erforschten Phänomen zu gewinnen.

Das Kapitel ist wie folgt aufgebaut. Zunächst entwickele ich ein theoretisches Verständnis von Zugang zu Land in traditionellen Landrechtssystemen. 
Dafür arbeite ich rechtebasierte sowie relationale und strukturelle Mechanismen heraus, die für den Zugang zu Land in traditionellen Landrechtssystemen relevant sind. Diese Mechanismen bilden gleichzeitig die Kategorien für die Analyse meiner Daten. In einem zweiten Schritt stelle ich Steven Lukes' Machtkonzept dar. Aus diesem leite ich die Analysekategorien der sichtbaren, versteckten und unsichtbaren Macht ab. Im Anschluss daran erläutere ich, wie sich die beiden Konzepte kombinieren lassen. Schließlich gehe ich der Frage nach, wie sich die zuvor herausgearbeiteten theoretischen Kategorien mit Blick auf die Analyse der empirischen Daten operationalisieren lassen. In diesem Rahmen ordne ich jeder Kategorie Indikatoren und Beispiele zu, die Anhaltspunkte dafür liefern, wie sich die theoretisch hergeleiteten Kategorien in der Auswertung der empirischen Daten erfassen lassen. In diesem Rahmen diskutiere ich ebenfalls Herausforderungen und Schwächen, welche die theoretischen Kategorien hinsichtlich der empirischen Analyse mit sich bringen sowie den Umgang damit.

\subsection{Zugang als analytische Kategorie in Konflikten um Land}

Die Konzeptualisierung von Zugang zu Land als Fähigkeit und nicht als Recht, einen Nutzen aus Land ziehen zu können, ermöglicht es Zugang zu Land unter Berücksichtigung sozialer Verhältnisse $\mathrm{zu}$ analysieren und nicht allein über de jure Besitzverhältnisse. Gesetze und sich daraus ableitende Rechte egal ob schriftlich oder mündlich festgehalten, staatliche oder traditionelle können den vielschichtigen Zugang zu Land nicht hinreichend abbilden (Ribot/Peluso 2003: 156). Zugang als analytische Kategorie ermöglicht es hingegen, ein vollständigeres Bild der Mechanismen zu zeichnen, die beeinflussen, ob Akteure einen Nutzen aus Land ziehen können (vgl. Ribot 1998). Die einzelnen Mechanismen determinieren jedoch nicht den Zugang zu Land, sondern bedingen sich gegenseitig oder komplementieren sich. Gemeinsam bilden sie - in den Worten von Ribot und Peluso (2003: 154)- so genannte Machtbündel (bundles of power), auf die Personen und Institutionen zurückgreifen können. Diese Machtbündel sind akteurs- sowie kontextabhängig und die Machtbündel von Individuen, Gruppen oder Institutionen verweben sich zu Netzen der Macht (webs of power) (ebd.: 154). Demnach variiert, je nach Kontext, auch die Stellung der Akteure in diesen Netzen der Macht. Die Ausgestaltung des Zugangs zu Land ändert sich mit politisch-ökonomischem und institutionellem Wandel. Sich verändernde politisch-ökonomische und insti- 
tutionelle Rahmenbedingungen beeinflussen soziale Verhältnisse und wirken sich dadurch auf die Möglichkeiten von Gruppen und Individuen, Zugang zu Land zu kontrollieren oder (aufrecht) zu erhalten, aus (ebd.: 158). Auf welche Mechanismen Akteure zurückgreifen können, um Zugang zu erlangen oder aufrechtzuhalten, ist kontextabhängig. Daraus leite ich die Annahme ab, dass sich im Kontext von land grabbing die Zugangsmechanismen möglicherweise verändern.

In traditionellen Landrechtssystemen ermöglichen, neben rechtebasierten Mechanismen, relationale und strukturelle Mechanismen den Zugang zu Land (Berry 1992, 2018; Peters 2004). Rechtebasierte Zugangsmechanismen sind alle legalen sowie illegalen Mittel, die Zugang zu Land ermöglichen. Legale Mittel umfassen Gesetze, Gewohnheitsrechte oder individuelle Vereinbarungen, die gegenüber Dritten eingefordert werden müssen. Illegale Mittel können Diebstahl, Zwang oder Erpressung sein und dienen dazu, direkten Zugang zu Land zu erlangen, ohne dass Dritte diesen als legitim anerkennen. Rechtebasierte Mechanismen können, müssen jedoch nicht zwangsläufig mit der Fähigkeit verbunden sein, einen Nutzen aus Land zu ziehen. In vielen Fällen bedingen oder komplementieren relationale und strukturelle Mechanismen die rechtebasierten Mechanismen. Relationale und strukturelle Mechanismen gehen aus den kontextspezifischen Bedingungen hervor, unter denen Akteure nach Zugang zu Land streben. Sie beeinflussen die Möglichkeiten von Akteuren, Zugang zu Land zu erlangen. Zu relationalen und strukturellen Mechanismen zählen Möglichkeiten der Aushandlung sozialer und rechtlicher Ordnungen, in dessen Rahmen Akteure nach Zugang zu Land streben, das Engagement in Netzwerken sowie in sozialen Beziehungen, die Zugang zu Land ermöglichen. Darüber hinaus beeinflussen unterschiedliche Kategorien sozialer Zugehörigkeit, die mit strukturell begründeten Ungleichheitsverhältnissen einhergehen, die Möglichkeiten, Rechte an Land einzufordern und Zugang zu Land zu erlangen. Dazu zählen insbesondere Herkunft, Geschlecht und Alter (Berry 1989, 1993, 2009b, 2018; Juul/Lund 2002a; Lund 2011, 2013; Moore 1978; Sikor/Lund 2009b). Aber auch vorhandene Ressourcen wie Bildung oder Kapital - können rechtebasierten Zugang zu Land in traditionellen Landrechtssystemen stärken oder ermöglichen (Ribot/Peluso 2003: 167ff.). Rechtebasierte Zugangsmechnismen sind in vielfacher Weise mit relationalen und strukturellen Zugangsmechanismen verschränkt, bedingen sich gegenseitig und komplementieren einander. Rechte an Land sind beispielswese oftmals an soziale Zugehörigkeit geknüpft oder unterschiedliche soziale Zugehörigkeit kann mit strukturellen Ungleichheitsverhältnissen - beispiels- 
weise in Bezug auf Bildung oder Kapital - einhergehen. Eingeschränkter Zugang zu Land aufgrund sozialer Zugehörigkeit kann wiederum durch Beziehungen verbessert werden.

Machtverhältnisse können über die analytische Kategorie der Kontrolle in den Blick genommen werden. Zugang $\mathrm{zu}$ Land $\mathrm{zu}$ kontrollieren, bedeutet, überwachen, regulieren und lenken zu können, wer - auf welche Weise auch immer - einen Nutzen aus Land ziehen kann. Dies ist Ausdruck von Macht gegenüber denjenigen, die nicht über diese Fähigkeit verfügen und dementsprechend Zugang zu Land nur über Akteure erlangen und aufrechterhalten können, die ihn kontrollieren. Wer Kontrolle über den Zugang zu Land ausüben kann, ist eng an bestehende Rechte und damit an Vorstellungen von Eigentum und Besitz geknüpft. Gesetze, Normen und sich daraus ableitende Rechte sind jedoch nicht einmal etabliert und damit gesetzt, sondern Gegenstand sozialer Aushandlungsprozesse (Moore 1978). »Existing orders are endlessly vulnerable to being unmade, remade and transformed, and [...] even maintaining and reproducing themselves, staying as they are, should be seen as a process" (ebd.: 6). Rechte sind also immer in ihrem räumlichen und zeitlichen Kontext zu verstehen, der sich auf die Fähigkeit, Kontrolle über Zugang zu Land auszuüben und Zugang zu Land aufrechtzuerhalten, auswirken kann. »Property is not about things but about relationships between and among persons with regard to things " (Moore 1998: 33). Kontrolle und Aufrechterhaltung von Zugang zu Land gestalten die Beziehungen zwischen den unterschiedlichen Akteuren in Bezug auf die Aneignung und Nutzung von Land. Sowohl in Bezug auf die Kontrolle als auch auf die Aufrechterhaltung des Zugangs zu Land ist es daher für die Akteure zentral, kontinuierlich in relationale Zugangsmechanismen zu investieren (vgl. Berry 1993). Investitionen in relationale Mechanismen sind vor allem der Aufbau und die Pflege sozialer Beziehungen oder Netzwerke. Diese können unterschiedliche Formen annehmen, beispielsweise die anteilige Übertragung von Gewinnen - wie Ernteerträgen - an Akteure, die Kontrolle über den Zugang ausüben oder die Gewährung von Vorzügen - wie Arbeit - um sich Anerkennung zu sichern. Eine Person kann in einer Situation den Zugang zu Land kontrollieren, in einer anderen aber darauf angewiesen sein, ihn über eine andere Instanz zu erlangen. Die Kontrolle des Zugangs zu Land kann zwischen unterschiedlichen kooperierenden oder konkurrierenden Akteuren aufgeteilt sein. Akteure, die Zugangsmechanismen kontrollieren, nehmen in Konflikten dementsprechend eine beherrschende Stellung gegenüber denjenigen ein, die nicht über diese Fähigkeit verfügen (Ribot/Peluso 2003: 159). 
Im Folgenden gehe ich näher auf die einzelnen Mechanismen ein, die $\mathrm{Zu}$ gang zu Land in traditionellen Landrechtssystemen ermöglichen.

\section{Rechtebasierte Zugangsmechanismen}

Rechtebasierte Zugangsmechanismen umfassen legale sowie illegale Mittel, über die Zugang zu Land gesichert wird. Legaler Zugang beruht auf Rechten, die sich aus Gesetzen, Gewohnheit oder Vereinbarung ableiten. Diese ermöglichen es den Akteuren, Zugang zu Land gegenüber Dritten einzufordern. In staatlichem Recht erfolgt dies beispielsweise unter Berufung auf Grundbucheinträge, Besitzurkunden, Lizenzen oder Konzessionen. In traditionellen Landrechtssystemen dienen Gewohnheitsrecht oder individuell vereinbarte (mündliche oder schriftliche) Abkommen als Grundlage für die Einforderung von Zugang zu Land. Gewohnheitsrecht und individuelle Vereinbarungen beruhen in der Regel auf der Anerkennung sozialer Normen und Regeln, denen sich beide Seiten verpflichten. Für die Durchsetzung der auf diese Weise erlangten Zugangsansprüche bedarf es somit keinem Zwang. Je nach Kontext kann sich jedoch die Kontrolle über den Zugang zu Land ändern, so dass auch in traditionellen Landrechtssystemen teilweise Zwangsmaßnahmen zur Durchsetzung von Rechten zum Einsatz kommen. Dazu zählen beispielsweise bewaffneter Widerstand im Kampf gegen Enteignung oder Vertreibung von gewohnheitsrechtlichem Land. Diejenigen, die nicht die Möglichkeit haben, Rechte auf Land geltend zu machen oder sie auszuhandeln, sind darauf angewiesen, Zugang zu Land über diejenigen $\mathrm{zu}$ erhalten, die ihn kontrollieren. Im Gegenzug erbringen sie in der Regel eine Geld- oder Naturalleistung. In Kontexten, die durch Rechtspluralismus geprägt sind, geht rechtebezogener Zugang nicht selten mit Widersprüchen einher. Denn neben dem Gewohnheitsrecht - das seinerseits schon umkämpft sein kann besteht in der Regel auch die Möglichkeit, sich auf staatliches Recht zu beziehen. Die unterschiedlichen Rechtsgrundlagen überschneiden sich häufig in ihrer Reichweite, und unterschiedliche Institutionen können rechtebasierten Zugang zu Land gewährleisten. Rechtspluralismus eröffnet damit einigen Akteuren die Gelegenheit des forum shopping (vgl. von Benda-Beckmann 2002). Dieses bezeichnet die Möglichkeit, eigene Anliegen und Vorhaben in dem formal- oder gewohnheitsrechtlichen Raum zu verhandeln, der die eigenen Ziele besonders begünstigt. Jenseits der Rechtssphäre gewinnen daher hinsichtlich der Frage, wer staatliches Recht, Gewohnheitsrecht oder Konventio- 
nen wann und mit welcher Absicht nutzen kann, politisch-ökonomische und institutionelle Rahmenbedingungen an Bedeutung (Ribot/Peluso 2003: 157).

Illegaler Zugang lässt sich über die Abgrenzung zu bestehenden Rechten definieren, die sich aus Gesetzen, Gewohnheit oder Vereinbarungen ableiten. Die Unrechtmäßigkeit ist jedoch eine Frage der Perspektive oder des Verhältnisses einer Person zu bestimmten Rechtsgrundlagen (ebd.: 164). Dies kann am Beispiel einer Landnutzerin illustriert werden, die gewohnheitsrechtliche Ansprüche auf Land geltend macht, indem sie eine neuerrichtete physische Barriere, wie einen Zaun überwindet, um auf dem zuvor gemeinschaftlich genutzten Land Feuerholz zu sammeln. Nach staatlichem Recht handelt sie eventuell illegal oder kriminell, da sie formalisierte Eigentumsrechte verletzt hat. Laut Gewohnheitsrecht würde ihr die Nutzung des Landes möglicherweise jedoch zustehen. Illegaler Zugang bedeutet demnach mithilfe von Mitteln, die nicht durch staatliches Recht oder gewohnheitsrechtliche Regeln und Normen legitimiert sind, einen Nutzen aus Land zu ziehen. In vielen Fällen ist illegaler Zugang mit Zwang, Gewaltandrohung oder manifester Gewalt verbunden. Illegale Mittel dienen sowohl zur Kontrolle als auch zur Aufrechterhaltung des Zugangs zu Land. Beispiele für illegalen Zugang zu Land sind der Diebstahl von Ernteerträgen, die Sicherung einer Landfläche mit Waffengewalt durch nicht-staatliche Akteure oder die Kontrolle des Zugangs durch Vortäuschen falscher Tatsachen. Im Kontext von land grabbing stellen Unternehmensakteure häufig Sicherheitskräfte ein oder errichten physische Barrieren, um den Zugang zu (umstrittenem) Land zu kontrollieren, obwohl sie (noch) nicht über formalisierte Rechte an dem Land verfügen.

\section{Relationale und strukturelle Zugangsmechanismen}

Relationale und strukturelle Zugangsmechanismen bilden eine zusammenhängende übergeordnete Analysekategorie. Sie umfasst Zugangsmechnismen, die Akteure innerhalb sozialer Beziehungen im Rahmen struktureller Grenzen aushandeln. Strukturelle Rahmenbedingungen verstehe ich als nicht deterministisch, sondern kontext- und akteursabhängig. Durch Aushandlung können sie sich verändern. Relationale und strukturelle Mechanismen, die den Zugang zu Land in traditionellen Landrechtssystemen bestimmen, sind soziale Zugehörigkeit, Netzwerke und soziale Beziehungen.

Soziale Zugehörigkeit bildet die Grundlage für den Zugang zu Land und geht einher mit strukturellen Ungleichheitsverhältnissen. Insbesondere in traditionellen Landrechtssystemen, in denen nicht-staatliche Akteure - wie 
geistliche Führer, Dorfvorsteher oder traditionelle Autoritäten - den Zugang zu Land kontrollieren, ist dieser in der Regel selektiv an Merkmale sozialer Zugehörigkeit geknüpft. Seit präkolonialer Zeit ist Zugang zu Land in vielen Gesellschaften Afrikas mit der Zugehörigkeit zu oder der Akzeptanz durch eine bestimmte Abstammungsgruppe, Familie oder Gemeinschaft beziehungsweise der Loyalität gegenüber traditionellen Autoritäten verknüpft (Berry 2018: 6). Noch heute ist der Zugang zu Land für Außenstehende von der erfolgreichen Verhandlung ihrer Gruppenmitgliedschaft oder der Anerkennung traditioneller Landrechtsinstitutionen abhängig (Berry 1993: 104). Mit Beginn der Kolonialherrschaft haben Kolonialverwalter*innen über ihre Interpretation des Gewohnheitsrechts zudem den Grundsatz etabliert, dass traditionell verwaltetes Land kein individuelles Eigentum, sondern gemeinschaftlicher Besitz ist. Über diese Interpretation traditioneller Landrechte knüpften Kolonialverwalter*innen Zugang zu Land grundsätzlich an die Zugehörigkeit zu einer Gemeinschaft. Welche Gemeinschaft Zugang zu welchem Stück Land kontrolliert, ließen sie jedoch offen. Die Konsequenz war eine Konkurrenz zwischen unterschiedlichen Gruppen sowie die Neuverhandlung sozialer Zugehörigkeit, mit dem Ziel Zugang zu Land zu sichern (Berry 1993: 40; Crook 1986: 89). Mit der Zeit hat sich diese Kodifizierung traditioneller Landrechte in vielen Gesellschaften strukturell als rechtliche und soziologische Tatsache verankert. Die Sicherung von Zugang zu Land erfolgt bis heute unter Rückbezug auf die vermeintliche Zugehörigkeit zu einer bestimmten Gruppe. Mit dem Ziel Zugang zu Land zu kontrollieren, beziehen sich konkurrierende Akteure oder Gruppen auf im Widerspruch zueinander stehende normative Ordnungen oder führen unterschiedliche vermeintliche Präzedenzfälle aus der Vergangenheit an, um die Hoheit zu erlangen, Rechte an Land zu bestimmen (Lund 2011: 72). Die Zugehörigkeit $\mathrm{zu}$ einer Gemeinschaft, die erfolgreich Ansprüche auf Land geltend macht, ist in vielen Fällen die Voraussetzung, um Rechte an Land zu verteidigen, zieht jedoch nicht automatisch Rechte nach sich oder garantiert Individuen Zugang zu Land. Denn Konkurrenz um den Zugang zu Land besteht nicht nur zwischen unterschiedlichen Gruppen, sondern auch innerhalb von Gruppen. Nicht alle Mitglieder einer Gruppe genießen in dieser auch die gleiche Zugehörigkeit. Die Zugehörigkeit zu einer Gruppe leitet sich zunächst daraus ab, als Teil der Gemeinschaft akzeptiert zu sein und ein langfristiges Interesse daran zu haben, Mitglied der Gemeinschaft zu sein. Dazuzugehören bedeutet, Werte, Netzwerke und Gepflogenheiten zu teilen, andere anzuerkennen und somit Teil des Sozialgefüges zu sein (Anthias 2006: 21f.; 
Pfaff-Czarnecka 2011: 5). Beispiele für Kriterien der Integration in die Gemeinschaft sind eine gemeinsame Sprache, gemeinsame Essgewohnheiten, Musik, eine gemeinsame Religion oder das Feiern bestimmter Feste (ArhinSam 2019: 35f.). »Belonging [...] combines (1) perceptions and performance of commonality; (2) a sense of mutuality and more or less formalized modalities of collective allegiance, and (3) material and immaterial attachments that often result in a sense of entitlement." (Pfaff-Czarnecka 2011: 2, Hervorhebung im Original). Hinsichtlich der Möglichkeiten, Zugang zu Land zu erlangen, bezieht sich soziale Zugehörigkeit jedoch nicht nur auf die Zugehörigkeit zu der Gruppe, die das Land vermeintlich erobert, als erste besiedelt oder bestellt hat. Ebenfalls bedeutsam für den Zugang zu Land sind Kategorien wie Alter, Geschlecht, Klasse, Religion und Herkunft. Insbesondere Herkunft - verhandelt mittels Kategorien wie Indigenität oder Autochthonie sowie first comer/late comer - ist relevant (Lund 2011: 74). Das bedeutet, dass Zugang $\mathrm{zu}$ Land nicht für jedes Mitglied einer Abstammungsgruppe gleichberechtigt ausgestaltet ist und de facto nicht der gemeinschaftlichen Kontrolle unterliegt. Wanderfeldbäuerinnen und -bauern, mobile Tierhalter*innen, die arme Landbevölkerung, junge Menschen und Frauen gehören zu den benachteiligten Personengruppen. Machtvollen Akteuren hingegen kommt die flexible Aushandlung zugute, indem sie traditionelle Besitzrechte in Übereinstimmung mit engen Gruppeninteressen neu definieren (Amanor 2012: 735). Während Zugehörigkeit zu einer Gruppe potenziell Rechte an Land ermöglicht, kann Nicht-Zugehörigkeit dazu führen, dass die Möglichkeit, Ansprüche geltend zu machen, von vornherein verwehrt bleibt. Der Bezug auf Kategorien sozialer Zugehörigkeit zur Sicherung des Zugangs $\mathrm{zu}$ Land ist somit zentral. Strukturelle Ungleichheitsverhältnisse führen jedoch dazu, dass sein Nutzen sich zwischen unterschiedlichen Akteuren unterscheidet. Darüber hinaus hängt er auch davon ab, wieviel Einfluss zentralstaatliche Institutionen in der Anerkennung, Überwachung und Auflösung von Landrechtsansprüchen haben und entsprechend, wie groß der Verhandlungsspielraum lokaler Akteure ist (Lund 2011: 74).

Um Zugang zu Land zu erlangen und $\mathrm{zu}$ festigen ist nicht nur die soziale Zugehörigkeit von Akteuren zentral. Relevant ist ebenfalls das Engagement in Netzwerken. Netzwerke können ortsgebundene Gemeinschaften oder professionelle und politische Interessensverbände sein. Viele Menschen engagieren sich in diversen Netzwerken zugleich und nutzen diese mit wechselnden Prioritäten (Berry 1993: 133). Das ermöglicht es, sich eine Vielzahl unterschiedlicher Verhandlungswege und -möglichkeiten offen zu halten, über die Zugang 
zu Land gesichert werden kann (Berry 1993: 14f.). Um Zugang zu Land aushandeln zu können, ist die Möglichkeit, Einfluss auf Debatten und Entscheidungsprozesse nehmen zu können, zentral. Einfluss ist wiederum eng mit der Fähigkeit, Anhänger*innen bzw. Unterstützer*innen mobilisieren zu können verbunden, wofür Netzwerke vorteilhaft sind. Netzwerke müssen also nicht zwangsläufig mit einem unmittelbaren Nutzen verbunden sein, sondern sind eher auf langfristige Sicht von Bedeutung. Es kann beispielsweise von Vorteil sein, auf die exklusive Kontrolle von Zugang zu Land zugunsten der potenziell wichtigen Mitgliedschaft in einem Netzwerk zu verzichten, um verschiedene Aushandlungsmöglichkeiten in Bezug auf Zugang zu Land aufrechtzuerhalten (Berry 1993: 14). Akteure richten ihre Aufmerksamkeit mal mehr auf das eine, mal mehr auf das andere Netzwerk, wodurch die kollektive Handlungsfähigkeit im Rahmen von Netzwerken zu- oder abnehmen kann. Unabhängig davon bleiben Netzwerke aber soziale Räume, welche die Wiederaufnahme oder Neuverhandlung bestimmter Themen ermöglichen (Moore 1986: 275). Netzwerke sind deshalb gleichermaßen für mächtige wie für weniger mächtige Akteure relevant. Mächtige Akteure, die sich in Aushandlungsprozessen durchsetzen können und folglich relativ sicheren Zugang zu Land haben, benötigen Netzwerke, um - dank zahlreicher Anhänger*innen - ihren Einfluss aufrechterhalten zu können. Für weniger mächtige Akteure ist das Engagement in Netzwerken nötig, um erfolgreich soziale Zugehörigkeit aushandeln und überhaupt an Debatten teilhaben zu können. Die Aushandlung sozialer Zugehörigkeit und damit einhergehend die Verhandlungsfähigkeit hängen von einer Kombination aus zugeschriebenen und errungenen Qualifikationen ab, für die das Engagement in Netzwerken notwendig ist. Ebenso wie die Verhandelbarkeit durch soziale Differenzierung beeinflusst wird, ist auch die Möglichkeit sich in Netzwerken zu engagieren mit Kategorien sozialer Zugehörigkeit verknüpft (Amanor 1999: 44). Um in Netzwerke investieren $\mathrm{zu}$ können und als legitimes Mitglied anerkannt $\mathrm{zu}$ werden, ist materieller Wohlstand wichtig (Berry 1989: 42).

Der dritte und letzte Zugangsmechanismus, den ich unter relationale und strukturelle Zugangsmechanismen fasse, sind Beziehungen. Dies können soziale Beziehungen innerhalb der Gemeinschaft sein oder der Zugang zu Behörden (Ribot/Peluso 2003: 170), je nachdem, wer den Zugang zu Land kontrolliert. Soziale Beziehungen innerhalb der Gemeinschaft sind für viele Landnutzer*innen ein relevanter Zugangsmechanismus. Akteure ohne Zugang zu Land, können diesen über Beziehungen zu Akteuren, die Zugang zu Land kontrollieren, erlangen. Eine weitverbreitete Form von 
Zugang zu Land mittels sozialer Beziehungen ist das landwirtschaftliche Produktionssystem des sharecropping. Sharecropping ist eine Form der Pacht und bedeutet, dass Akteure, die Zugang zu Land kontrollieren, Pächter*innen (sharecropper) ihr Land für die Bewirtschaftung zur Verfügung stellen. Im Gegenzug erhalten sie einen vorher vereinbarten Anteil der erwirtschafteten Ernte, wodurch beide Parteien einen Nutzen aus dem Land ziehen können. Ebenso besteht auch die Möglichkeit, Zugang zu Land über eine klassische Pachtvereinbarung zu erhalten, in dessen Rahmen Akteuren Land gegen eine Geldleistung nutzen können. Beziehungen können darüber hinaus Zugang zu Land über die Vermittlung von Arbeitsplätzen ermöglichen. Der Nutzen dabei ist beidseitig. Im Sinne klientelistischer Beziehungen können Personen, die Arbeitsplätze kontrollieren, diese im Austausch diverser Gefälligkeiten vermitteln. Die Vermittlung von Arbeitsplätzen kann gleichzeitig auch als Investition in Netzwerke dienen, die zu einem späteren Zeitpunkt möglicherweise von Vorteil sind - auch um Zugang zu Land zu kontrollieren (Ribot/Peluso 2003: 167). Neben persönlichen Beziehungen innerhalb der Gemeinschaft sind, insbesondere mit Blick auf die Kontrolle über Zugang zu Land, auch Beziehungen zu unterschiedlichen Institutionen relevant. Die Beziehung zu diesen unterscheidet sich zwischen Akteuren, in Abhängigkeit wirtschaftlicher und sozialer Voraussetzungen. Beispielsweise sind Behörden oftmals weit entfernt und nicht jede* $r$ hat die entsprechenden Mittel - Geld, Transportmöglichkeiten, Zeit, Wissen - um sie aufzusuchen. Zudem konkurrieren Beamt*innen in Justiz- und Verwaltungsbehörden sowie traditionelle Autoritäten teilweise um sich überschneidende Zuständigkeitsbereiche. Akteure mit entsprechendem Wissen können dies im Sinne des forum shopping - der Möglichkeit, eigene Anliegen in dem formal- oder gewohnheitsrechtlichen Raum zu verhandeln, der die eigenen Ziele besonders begünstigt - zu ihrem eigenen Vorteil nutzen und sich über unterschiedliche Wege Zugang zu Land erschließen. Wissen - in diesem Fall Kenntnisse der eigenen Rechte oder Regeln der Landnutzung - kann auf ein hohes Bildungsniveau oder die Mitgliedschaft in Netzwerken, die Informationen zur Verfügung stellen, zurückzuführen sein. Akteure, denen entsprechendes Wissen fehlt, haben auch nicht die Möglichkeit des forum shopping. So kann eine wohlhabende Landnutzerin mit hohem Bildungsniveau, die aufgrund ihrer sozialen Zugehörigkeit traditionelle Rechte an Land erworben hat, diese dank ihres Wissens und ihrer finanziellen Mittel über Behörden formalisieren lassen, um sie zu stärken. Ein armer, wenig gebildeter Landnutzer hat diese Möglichkeit vielleicht nicht. Indessen unterhält er aufgrund seiner Loyalität 
aber möglicherweise eine gute Beziehung zu dem chief, der den Zugang zu Land kontrolliert. Diese Beziehung kann ebenfalls privilegierten Zugang zu Land ermöglichen, z.B. über die Vermittlung von Arbeit. Eine migrantische Landnutzerin hat vielleicht weder die eine, noch die andere Möglichkeit.

Bildung und Wissen sind auch über die Kenntnisse der eigenen Rechte hinaus relevant für den Zugang zu Land. Besonders deutlich wird dies an so genannten Expert*innen. Ein Expert"innenstatus - den Akteure über exklusive Informationen, spezialisiertes Wissen oder Hochschulabschlüsse mit ihren entsprechenden Titeln erlagen - kann Zugang zu Land in unterschiedlicher Weise beeinflussen. Die dem Expert*innentum zugeschriebene Kompetenz geht in vielen Fällen damit einher, Einfluss auf andere Akteure oder auf Diskurse, Normen und Regeln ausüben zu können. Dies kann wiederum Auswirkungen auf den Zugang zu Land haben. Beispielsweise rechtfertigen "wissenschaftliche« Narrative, die auf dem Wissen vermeintlicher Expert" innen beruhen, die großflächige Aneignung von Land durch Unternehmensakteure. Darüber hinaus kann exklusives Wissen in Form von Informationen dem eigenen Vorteil in Bezug auf Zugang zu Land dienen. Akteure, die $\mathrm{Zu}$ gang zu Land kontrollieren, können anderen Akteuren Informationen vorenthalten, um Widerstand zu vermeiden und die eigene Kontrolle des Zugangs aufrechtzuerhalten. Wissen kann ebenfalls ermöglichen, errungene Gewohnheitsrechte an Land über rechtliche Schritte aufrechtzuhalten und gegen den Verlust von Land zu kämpfen. Kenntnisse der eigenen Rechte und derjenigen anderer Akteure wirken sich auf den eigenen Handlungsspielraum aus. Nur wer die unterschiedlichen Möglichkeiten Zugang zu Land zu erlangen und zu kontrollieren, kennt, kann sie auch nutzen oder andere davon ausschließen. Soziale Zugehörigkeit, Netzwerke, soziale Beziehungen und strukturell begründete Ungleichheitsverhältnisse hängen zusammen und wirken sich auf die Kontrolle des Zugangs und den Zugang zu Land aus.

Soziale Zugehörigkeit, Netzwerke und soziale Beziehungen sind wandelbar und können sich - innerhalb struktureller Grenzen - im Laufe der Zeit über soziale Aushandlungsprozesse verändern, was sich wiederum auf den Zugang zu Land auswirken kann. Diese Verhandelbarkeit ist Gegenstand einer umfangreichen Literatur aus der Rechtsanthropologie und der historischen Sozialforschung. Studien, die sich in diesem Forschungsfeld verorten, verweisen darauf, dass traditionelle Landrechtssysteme in post-kolonialen Staaten Subsahara-Afrikas nicht statisch sind und auf fortbestehenden präkolonialen Regeln und Normen beruhen, sondern auf Basis der Interpretation von Tradition durch die Kolonialverwalter*innen entstanden sind (Chanock 
1985; Moore 1978; Ranger 1983). Es handelt sich also um neue Rechtssysteme die aus der kontextbezogenen Auslegung sozialer und rechtlicher Ordnungen heraus entstanden sind. Die Auslegung sozialer und rechtlicher Ordnungen in Bezug auf den jeweiligen relevanten Kontext ist nicht außergewöhnlich. Ganz im Gegenteil: die Ausübung jeglicher Rechtspraxis durch Jurist*innen besteht darin, rechtliche Grundlagen mit Bezug auf den jeweiligen Kontext zu interpretieren (Moore 1978: 1). Dies gilt auch für traditionelles Recht während der Kolonialzeit. Je nachdem welche Person an welchem Ort und zu welchem Zeitpunkt Auskunft darüber gab, war es Gegenstand unterschiedlicher Interpretation (Berry 2002: 645). Dies hat sich bis heute nicht geändert. Die Bedeutung und Auslegung bestimmter Konzepte des traditionellen Rechts bleiben Gegenstand sozialer Aushandlungsprozesse.

»Customs are constantly recreated and disputed. Men's custom is not always women's, elders' may not be juniors', and the tradition of the rich may differ from those of the poor - or be made to look as though they do « (Shipton/Coheen 1992: 308).

Verschiedene Individuen oder Gruppen schreiben $\mathrm{zu}$ unterschiedlichen Zeitpunkten und in unterschiedlichen Kontexten allgemein bekannten Konzepten, Begebenheiten oder Handlungen variierende Definitionen oder Bedeutungen $\mathrm{zu}$, um eigene Interessen durchzusetzen (Lund 2013; Peters 1984). Damit ist Verhandelbarkeit ein zentrales Merkmal von Gewohnheitsrechten (Berry 1993: 14). In Konflikten um Zugang $\mathrm{zu}$ Land ist Verhandelbarkeit als analytische Kategorie von besonderer Relevanz. Mit entsprechendem Geschick erweist sich die Mehrdeutigkeit des Gewohnheitsrechts für Konfliktakteure*innen als zentraler Vorteil, welcher der Verfolgung eigener Ziele dienen kann. Gleichzeitig können sich durch Verhandelbarkeit aber auch Widersprüche ergeben, die durch inkohärente oder inkompatible Ideen und Handlungen im Zusammenhang mit traditionellem Recht entstehen. Ein Beispiel für widersprüchliche Bedeutungszuschreibungen von Konzepten ist der Bezug auf Tradition selbst. Akteure können sich auf Tradition berufen, um gegenwärtig ausgeübte Vorgehensweisen zu legitimieren. Tradition kann als ein Vorbild dargestellt werden, dem es zu folgen gilt. Sie kann jedoch auch als ein veraltetes Model kritisiert werden, das es zu überwinden gilt (Peters 1984: 30). Widersprüche eröffnen potenziell neue Aushandlungsräume: Die Fähigkeit Zugang zu Land zu erlangen, steht in unmittelbarem Verhältnis $\mathrm{zu}$ der geschickten - auf die eigenen Ziele ausgerichteten - Interpretation von Gewohnheitsrecht. So ist die Bedeutung von Kategorien sozialer Zu- 
gehörigkeit für den Zugang zu Land oftmals diskursiv erzeugt. Diskursive Konstruktionen können inklusiver oder exklusiver Art sein. Sie können dazu dienen, den eigenen Zugang $\mathrm{zu}$ Land $\mathrm{zu}$ ermöglichen oder ihn anderen Individuen oder Gruppen zu verwehren. Diskursive Strategien, die dazu dienen Zugang zu Land neu zu verhandeln oder aufrechtzuerhalten, können konkurrierende Identitätsdiskurse, die Wiederbelebung, Erfindung oder das Nacherzählen von Geschichte sein (Lund 2013). Strategien der Exklusion zeichnen sich häufig durch Bestrebungen aus, vormals flexible Gruppengrenzen - bezüglich Familie, Abstammungslinie, Dorf, ethnisch definierten Gemeinschaften oder anderen gesellschaftlichen Einheiten - festzuschreiben. Damit geht eine engere Definition sozialer Zugehörigkeit einher. In Konflikten um Land ergibt sich daraus eine stärkere Abgrenzung der Rechteinhaber*innen gegenüber denjenigen, denen Rechte verwehrt bleiben (Peters 2004: 302ff.). Verhandelbarkeit kann sich somit positiv und negativ auf Zugang zu Land auswirken. Verhandelbarkeit dient nicht jeder Person oder Gruppe gleichermaßen, sondern sie steht in engem Zusammenhang mit bestehenden Sozialstrukturen (Amanor 1999: 44). Die erfolgreiche Auslegung von Tradition und damit der Regeln und Normen, die den eigenen Zugang $\mathrm{zu}$ Land bestimmen, ist an Kategorien sozialer Zugehörigkeit geknüpft. Bestehende Sozialstrukturen, so argumentiert Amanor (ebd.), zwingen die meisten Menschen dazu, der Interpretationen dessen, was machtvolle Akteure als gewohnheitsrechtlich definiert haben oder neu definieren, zu folgen. Wer sich an entsprechenden Debatten beteiligen und Diskurse beeinflussen kann, ist eine Frage von Macht und sozialer Differenzierung. In Bezug auf die relationalen Zugangsmechanismen soziale Zugehörigkeit, Beziehungen und Netzwerke ermöglicht die Berücksichtigung von Verhandelbarkeit eine differenziertere Analyse sozialer Verhältnisse.

Wer auf welche Zugangsmechanismen zurückgreifen und darüber $\mathrm{Zu}$ gang zu Land erlangen, aufrechterhalten oder kontrollieren kann, hängt von sozialer Differenzierung und Machtverhältnissen ab. Gleichwohl die Kategorien der relationalen und strukturellen Zugangsmechanismen soziale Differenzierung berücksichtigen, fehlt ihnen ein erklärendes Moment, das Aufschluss darüber gibt, woher sich die Fähigkeit einzelner Akteure, Zugang $\mathrm{zu}$ Land $\mathrm{zu}$ kontrollieren - oder mit anderen Worten ihre Macht in Konflikten um Land -ableitet. Mit dem Ziel dies zu analysieren, nutze ich ein Machtkonzept, das Akteurshandeln in Beziehung zu strukturellen Faktoren wie politisch-ökonomischen und institutionellen Rahmenbedingungen, 
gesellschaftlich vorherrschenden Normen sowie internalisierten Denk- und Handlungsmustern setzt.

\subsection{Macht als analytische Kategorie in Konflikten um Zugang zu Land}

Mit dem Ziel analysieren zu können, über welche Handlungsstrategien es traditionellen Autoritäten gelingt, Zugang zu Land zu kontrollieren und wie sich diese Fähigkeit erklären lässt, verknüpfe ich die unterschiedlichen analytischen Kategorien des Zugangs mit dem von Steven Lukes (2005) entwickelten Konzept zur Analyse von Machtverhältnissen. Lukes unterscheidet zwischen drei Auffassungen von Macht, die er als eindimensionale, zweidimensionale und dreidimensionale Perspektive bezeichnet (ebd.: 15). Andere Autor*innen, die an Lukes' Konzept anschließen, bezeichnen diese drei Perspektiven als sichtbare (visible), versteckte (hidden) und unsichtbare (invisible) Macht (vgl. Gaventa 2006; VeneKlasen/Miller 2002). Diese Bezeichnungen übernehme ich im Folgenden. Sichtbare, versteckte und unsichtbare Macht schließen sich nicht gegenseitig aus, sondern bauen aufeinander auf und ergänzen sich.

\section{Sichtbare Macht}

Das Verständnis von Macht, das ich im Folgenden als sichtbare Macht bezeichne, geht insbesondere auf Arbeiten so genannter pluralistischer Theoretiker wie Robert Dahl (1957, 1961) oder Nelson Polsby (1960), zurück. Sie fassen Macht als formelle Entscheidungschancen, die von jeder Position innerhalb der Sozialstruktur gleichermaßen zugänglich, also pluralistisch verteilt sind (Offe 1977: 8). Aus dieser Perspektive ist Macht nicht in den Händen einer kleinen Elite gebündelt, sondern verteilt sich auf verschiedene gesellschaftliche Gruppen und Individuen (Lukes 2005: 5). Das erklärt sich darüber, dass Macht immer an Gegenstände geknüpft ist, die vergänglich oder andauernd sind und zu kurz- oder auch längerfristigen Koalitionen zwischen unterschiedlichen Akteuren führen können, jedoch nicht zeitlos oder unveränderlich sind (Polsby 1960: 478f.), Theoretisch schließt dieses Machtverständnis an den repressiven Machtbegriff nach Max Weber (1972 [1922]) an. Weber definiert Macht als "jede Chance innerhalb einer sozialen Beziehung den eigenen Willen auch gegen Widerstreben durchzusetzen« (1972 [1922]: 28). In Anlehnung daran beschreibt Dahl (1961) Macht als die Fähigkeit einer Person, 
eine andere Person dazu zu bringen etwas zu tun, das sie sonst nicht tun würde (Dahl 1957: 202f.). Dahl spezifiziert darüber hinaus, dass Macht den erfolgreichen Versuch einer Person, eine andere Person dazu zu bringen etwas zu tun, das sie sonst nicht tun würde, bedeutet (ebd.: 204). Diese Spezifizierung verweist auf einen zentralen Punkt in der Debatte um Macht, nämlich auf die Unterscheidung zwischen möglicher und tatsächlicher Macht, zwischen dem Machthabe und Machtausübung (Lukes 2005: 17). Das Konzept der sichtbaren Macht konzentriert sich auf letztere. Eine Untersuchung von Machtverhältnissen aus dieser Perspektive beschränkt sich auf die Untersuchung von Akteurshandeln. Ein beliebter Untersuchungsgegenstand, der dies ermöglicht, sind Entscheidungsfindungsprozesse zu Themen, über die es einen beobachtbaren Interessenskonflikt gibt. Macht bedeutet in diesem $\mathrm{Zu}$ sammenhang, in der Lage zu sein, Entscheidungen zu umstrittenen Themen gegen Widerstreben durchzusetzen (ebd.: 19). Eine solche Analyse von Machtverhältnissen setzt die Annahme voraus, dass Interessen bewusst sind und kommuniziert werden. In Anlehnung an Hartwig Schuck (2014: 304) bezeichne ich diese Interessen als manifeste Interessen. Damit grenze ich mich bewusst von Lukes missverständlicher Bezeichnung der subjektiven Interessen ab. Manifeste Interessen sind somit Bestrebungen oder Präferenzen, die Akteure formulieren und/oder die sich aus ihren Handlungen ablesen lassen, "gleichviel auf welchen ursprünglichen Motiven und Ursachen sie beruhen mögen« (ebd.). Über das Handeln gemäß (widersprüchlicher) Interessen und ihrer Kommunikation zeigt sich ein für Forschende beobachtbarer Konflikt, in dem Machtverhältnisse in Form von Entscheidungen zu strittigen Themen oder umkämpften Gegenständen deutlich werden.

\section{Versteckte Macht}

Der Fokus auf der tatsächlichen Ausübung von Macht anhand der Durchsetzung von Entscheidungen ist Gegenstand der Kritik des Konzepts der sichtbaren Macht (Bachrach/Baratz 1962: 948).

Ausgehend von dieser Kritik erweitern Peter Bachrach und Morton S. Baratz das Konzept der sichtbaren Macht um ein »zweites Gesicht« (1962) und damit um ein Verständnis von Macht, das ich in Anlehnung an John Gaventa (2006) sowie Lisa VeneKlasen und Valerie Miller (2002) als versteckte Macht bezeichne. Denn Macht, so argumentieren Bachrach und Baratz, bedeutet nicht ausschließlich, Entscheidungen gegen Widerstreben treffen $\mathrm{zu}$ können, sondern auch, mögliche alternative Entscheidungen, die den eige- 
nen Interessen entgegenstehen, von vornherein auszuschließen. In diesem Sinne umfasst Macht ebenfalls die Fähigkeit, politische und soziale Werte oder institutionelle Verfahrensweisen zu begünstigen, die dazu beitragen, dass nur solche Themen auf die politische Agenda oder in die öffentliche Diskussion gelangen, die nicht im Widerspruch zu den eigenen Interessen stehen bzw. diese nicht gefährden. Dadurch bekommen Gegner*innen für wichtige Anliegen keine politische Bühne und es wird ihnen so erschwert, öffentliche Aufmerksamkeit für umstrittene Themen zu gewinnen (ebd.: 948). Die Ausübung versteckter Macht umfasst darüber hinaus, bestimmen oder beeinflussen zu können, wer auf welche Weise an Entscheidungsprozessen teilnehmen kann und wer nicht. Die Analyse versteckter Machtverhältnisse untersucht, welche Themen gar nicht erst zur Diskussion stehen und daher auch nicht zum Gegenstand von Entscheidungen werden. Denn »[a]n dem Punkt eines Machtprozesses, an dem [die Analyse der sichtbaren Macht] ihre Beobachtungen aufnimmt, ist sozusagen das Wichtigste schon passiert: die Aussonderung von Alternativen, die dann auf der Ebene manifesten Entscheidungshandelns und beobachtbaren Widerstrebens keine Rolle mehr spielen« (Offe 1977: 14f.). Im Zentrum der Analyse versteckter Macht stehen damit die - wie Bachrach und Baratz es ausdrücken - »Nicht-Entscheidungen« (nondecisions) (1962: 949), die als Hinweis auf die Ausübung versteckter Macht gelten. Mit dem Begriff Nicht-Entscheidungen ist gemeint, dass machtvolle Akteure Widersprüche in den Einstellungen von Akteuren und damit potenzielle Konflikte - die Forschende zu identifizieren haben - strategisch aus Entscheidungsprozessen heraushalten und damit verhindern, dass sie institutionell ausgehandelt werden und Eingang in öffentliche Debatten finden. NichtEntscheidungen sind damit aktive Entscheidungen, die Aushandlung konfliktiver Themen zu vermeiden (Bradshaw 1976: 124). Nicht-Entscheidungen sind somit empirisch beobachtbare Entscheidungen, die dazu führen, dass latente oder manifeste Herausforderungen etablierter Werte oder Interessen von Entscheidungsträger*innen verdrängt oder ausgebremst werden. Sie sind ein Mittel, um Forderungen nach Veränderung - z.B. in Bezug auf die Verteilung von Privilegien - im Keim zu ersticken oder verdeckt zu halten, bevor sie ausgesprochen werden oder relevante Entscheidungsräume erreichen können (Bachrach/Baratz 1970: 44). In Hinblick darauf ist ein weiterer zentraler Punkt für die Analyse versteckter Macht die Berücksichtigung der vorherrschenden Werte, Überzeugungen und institutionellen Verfahrensweisen - kurz der "Spielregeln« - die sich zugunsten bestimmter Akteure und auf Kosten anderer auswirken (Bachrach/Baratz 1962: 950). Denn alle Formen 
politischer Organisation tendierten dazu, bestimmte Konflikte zu nutzen und andere auszublenden. Diese Tendenz bezeichnet Elmer Eric Schattschneider als mobilization of bias: "Some issues are organized into politics while others are organized out« (1960: 71).

Im Gegensatz zu dem Konzept der sichtbaren Macht beinhaltet dasjenige der versteckten Macht ein breiteres Verständnis von Interessen. Im Konzept der sichtbaren Macht sind Interessen die politischen Präferenzen, die sich in Entscheidungsprozessen - an denen vermeintlich jede* $r$ teilhaben kann - abzeichnen. Das Konzept der versteckten Macht berücksichtigt darüber hinaus die Interessen derjenigen, die teilweise oder gänzlich von Entscheidungsprozessen ausgeschlossen sind. Forschende können diese Interessen anhand offener oder verdeckter Missstände, auf die Akteure hinweisen, identifizieren. Offene Missstände sind solche, die Akteure öffentlich äußern und die bereits Diskussionsgegenstand der politischen Agenda sind. Verdeckte Missstände sind solche, die Akteure benennen, die es jedoch noch nicht in die öffentliche Aufmerksamkeit oder gar in politische Entscheidungsräumen geschafft haben (Bachrach/Baratz 1970: 49). Trotz dieses breiteren Verständnisses von Interessen folgen Bachrach und Baratz jedoch weiterhin der Annahme, Interessen würden bewusst als Bedürfnisse oder Präferenzen formuliert und seien beobachtbar (Lukes 2005: 24).

An diesem Punkt setzt Lukes mit seiner Kritik an, die drei zentrale Punkte umfasst. Er kritisiert erstens, dass das Konzept der versteckten Macht mit seinem Fokus auf beobachtbares (Konflikt-)Handeln ebenso wie das der sichtbaren Macht die Ausübung von Macht am Widerstreben unterlegener Akteure ausmache und damit Machtverhältnisse übersähe, die in Inaktivität unterlegener Akteure resultieren. Dies sei problematisch, da die Ausrichtung eines Systems nicht allein über die Handlungen einzelner Individuen oder Gruppen aufrechtgehalten wird, sondern insbesondere über sozial und kulturell strukturiertes Verhalten oder institutionelle Verfahrensweisen. Inaktivität könne diese Strukturen zusätzlich festigen. Zweitens kritisiert Lukes die Annahme, dass für die Analyse von Machtverhältnissen ein beobachtbarer Konflikt vorhanden sein muss. Denn Macht werde nicht nur ausgeübt, wenn eine Person eine andere Person dazu bringt, etwas zu tun, das sie ansonsten nicht tun würde. Macht auszuüben bedeute auch, die Interessen und Bedürfnisse einer anderen Person so zu prägen, $\mathrm{zu}$ beeinflussen und letztendlich $\mathrm{zu}$ bestimmen, dass Missstände und die ihnen zugrundeliegenden Widersprüche erst gar nicht als solche wahrgenommen und erkannt würden. Alternativen zum Status quo würden in diesem Fall nicht in Betracht gezogen, da dieser 
als selbstverständlich, unveränderbar oder vorteilhaft wahrgenommen würde (ebd.: 28). Auf diese Weise wirkten unterlegene Personen willig an ihrer Unterdrückung mit. Macht zeige sich damit nicht nur in manifesten Konflikten, sondern auch in ihrer Verhinderung oder Unterdrückung (ebd.: 27). Lukes dritter Kritikpunkt betrifft die implizite Annahme, auf der das Konzept der versteckten Macht beruht, dass Nicht-Entscheidungen nur dann vorliegen, wenn Missstände und damit eindeutig gegensätzliche Interessen identifiziert werden können, die nicht in Form von Themen Eingang in Entscheidungsräume finden. Das müsse im Umkehrschluss bedeuten, dass die vermeintliche Abwesenheit von Missständen bedeute, dass es auch keine Interessen gibt, die durch die Ausübung von Macht verletzt werden könnten (ebd.: 28).

\section{Unsichtbare Macht}

Anknüpfend an seine Kritik entwickelt Lukes das Konzept der unsichtbaren Macht, das über beobachtbares (Konflikt-)Handeln hinausgeht und damit die Konzepte der sichtbaren und versteckten Macht ergänzen soll. Eine Analyse der unsichtbaren Macht ist dazu bestimmt, Faktoren zu ermitteln, die Akteure daran hindern, Missstände und die ihnen zugrundeliegenden widersprüchlichen Interessen als solche zu erkennen. Damit bleiben auch die Mechanismen, die bewirken, dass potenzielle Themen gar nicht erst auf die Agenda gelangen, im Verborgenen. Um unsichtbare Macht in den Blick nehmen zu können, ist es notwendig, gleichermaßen gesellschaftliche Kräfteverhältnisse, institutionelle Verfahrensweisen und individuelles Handeln in Form von Entscheidungen zu berücksichtigen. Dies kann im Kontext eines manifesten, beobachtbaren Konflikts oder in seiner Abwesenheit geschehen; denn die Ausübung versteckter Macht kann einen manifesten Konflikt ggf. verhindern. Implizit bleibt dann ein latenter Konflikt bestehen. Relevant für die Analyse unsichtbarer Macht ist das Vorhandensein eines latenten Konflikts. Dieser äußert sich anhand eines Widerspruchs zwischen den Interessen der Machtausübenden und den, wie Lukes sie bezeichnet, »wirklichen« Interessen (real interests) derjenigen, die aufgrund von Machtverhältnissen ausgegrenzt werden. Letztere müssen sich laut Lukes - ebenfalls auf Grund existierender Strukturen und Machtverhältnisse - ihrer Interessen nicht bewusst sein oder sie äußern (ebd.). Dies lässt vermuten, es handele sich um Interessen, deren Identifizierung nicht durch die betroffenen Personen selbst erfolgen könne, jedoch sehr wohl durch Außenstehende. In der Rezeption von Lukes Konzept der unsichtbaren Macht interpretieren einige Autor*innen 
daher »wirkliche« als »objektive« Interessen im marxistischen Sinn (vgl. u.a. Benton 1981; Dowding 2006). Im klassischen Marxismus sind Interessen gleichbedeutend mit kollektiven Interessen oder Klasseninteressen. Konflikte sind aus dieser Perspektive auf widersprüchliche Interessen zurückzuführen, die sich aus der ungleichen Verteilung von Ressourcen und Macht ergeben (Turner 1975). Interessen werden in diesem Zusammenhang als »objektiv« gegeben angenommen. Entsprechend können Interessen Akteuren von außen zugeschrieben werden, ohne dass sie sich ihrer selbst bewusst sein müssen. Die Annahme solcher »objektiver « Interessen wurde vielfach als teleologisch, essentialistisch und reduktionistisch kritisiert (Sibeon 2004). Demgegenüber vertritt Lukes die Ansicht, Personen oder Gruppen, deren »wirkliche« Interessen durch die Ausübung von Macht verletzt werden, müssten eben diese Interessen selbst identifizierten können, insofern sie nicht durch Machtverhältnisse daran gehindert würden (Lukes 2005: 37). Sein Verständnis von »wirklichen« Interessen unterscheidet sich damit vom marxistischen Begriff der »objektiven« Interessen. Allerdings argumentiert Lukes an späterer Stelle, um unsichtbare Macht überhaupt erkennen $\mathrm{zu}$ können, bedürfe es eines normativen Urteils von außen (ebd.: 146). Ted Benton (1981) bezeichnet dies als paradox of emancipation. Damit bezieht er sich auf die Frage, wie eine Selbst-Emanzipation (oder bei Lukes die Identifizierung der wahren Interessen durch die Akteure selbst) erfolgen kann, wenn angenommen wird, dass die soziale Ordnung, in der die Akteure leben, ihr Bewusstsein beeinflusst bzw. systematisch manipuliert (ebd.: 162). Lukes selbst liefert keine Lösung für dieses Problem. Mit Blick auf die Analyse bleibt die Identifizierung der »wirklichen« Interessen damit schwierig.

Einen möglichen Lösungsansatz für diese Problematik bietet Schuck (2014) an. Er spricht sich für den Begriff der »ethischen Interessen« aus. Unter ethischen Interessen versteht er solche, die Akteuren »ein gutes Leben ermöglichen (würden)« (ebd.: 305). Diese ergeben sich, so argumentiert Schuck, aus »einer adäquaten kritischen Analyse der herrschenden Verhältnisse« (ebd.: 306) und aus "Annahmen über die menschliche Natur und das gute Leben« (ebd.: 306). Damit ist die Ermittlung von Interessen weiterhin abhängig von »ethischen Prämissen und Hypothesen bzw. - im historischen Rückblick - kontrafaktischen Entwicklungsverläufen« (ebd.: 306), auf die ich später im Abschnitt zur Operationalisierung der theoretischen Kategorien näher eingehe. Die Behauptung, dass entgegen der ethischen Interessen einer Person gehandelt wurde, impliziert die Annahme, dass die Handlung dem guten Leben dieser Person schadet. 
Tabelle 1: Übersicht über die drei Dimensionen der Macht

\section{Sichtbare Macht}

$\begin{array}{lll}\text { Betrachtung von... } \quad . & \text { Handeln } \\ & \text { Entscheidungsfindung } \\ . & \text { (zentralen) Themen } \\ . & \text { beobachtbaren (manifesten) Konflikten } \\ \text { manifesten Interessen, die Akteure formulieren und/oder die } & \text { sich aus ihren Handlungen ablesen lassen }\end{array}$

Versteckte Macht

Betrachtung von... . Entscheidungsfindung und »Nicht-Entscheidungen«

- Themen und potenziellen Themen

- beobachtbaren (manifesten und versteckten) Konflikten

- (manifesten) Interessen in Form politischer Präferenzen oder Missstände

\section{Unsichtbare Macht}

Betrachtung von... $\quad$. Entscheidungsfindung und Kontrolle über Agenda-Setting (nicht notwendigerweise anhand von Entscheidungen)

- Themen und potenzielle Themen

- beobachtbaren (manifesten und versteckten) sowie latenten Konflikten

manifesten und ethischen Interessen

Quelle: Eigene Darstellung in Anlehnung an Lukes (2005: 29)

\subsection{Zwischenfazit}

Um $\mathrm{zu}$ analysieren, in welchem Zusammenhang das Handeln traditioneller Autoritäten mit Konflikten um Zugang zu Land stehen, erweist sich die Kombination theoretischer Kategorien von Zugangsmechanismen mit einem dif- 
ferenzierten mehrdimensionalen, auf die empirische Analyse ausgerichteten Verständnis von Macht aus unterschiedlichen Gründen als sinnvoll.

Zugang als analytische Kategorie ermöglicht es, nicht nur bestehende Rechte an Land in der Untersuchung zu berücksichtigen, sondern den analytischen Blick zu weiten und alle Mechanismen $\mathrm{zu}$ erfassen, die Akteure befähigen, Zugang zu Land zur erlangen, also- auf welche Weise auch immer - einen Nutzen aus dem Land zu ziehen. Diese Mechanismen können in Konflikten um den Zugang zu Land Gegenstand von Aushandlungsprozessen und damit umstritten sein. Zugangsmechanismen sind strukturierende Elemente in Konflikten um Land und stellen als solche wichtige Ansatzpunkte für die Analyse von Konflikten um Land dar. In vielen Fällen lässt sich entlang von Zugangsmechanismen und ihrer Veränderung im Kontext von Transformationsprozessen nachvollziehen, worum es in den Konflikten geht. Darüber hinaus erlaubt es die Analyse von Zugangsmechanismen, eine Vielzahl möglicher Widersprüche zu erfassen, die auf die Heterogenität der Konfliktakteure und ihres Zugangs zu Land zurückgehen und Konflikten zugrunde liegen.

Jenseits der Analyse, über welche Mechanismen unterschiedliche Personen Zugang zu Land erlangen, liegt das zentrale Interesse meiner Studie auf der Kontrolle des Zugangs und der Frage, wie Kontrolle erlangt, erweitert und aufrechterhalten wird. Die Kontrolle des Zugangs zu Land ist in diesem $\mathrm{Zu}$ sammenhang als Ausdruck von Macht zu verstehen.

In der Vergabe und Kontrolle von traditionell verwaltetem Land sind traditionelle Autoritäten zentrale Akteure. Der analytische Fokus liegt daher auf der Macht traditioneller Autoritäten und den damit zusammenhängenden Möglichkeiten, Zugang zu Land zu kontrollieren. Da ich Macht als relational verstehe, ist die Macht traditioneller Autoritäten in Konflikten um Zugang zu Land immer in Bezug zu anderen Konfliktakteuren zu sehen. Für die Analyse bedeutet das, die unterschiedlichen Akteure in ihrem Verhältnis zueinander zu sehen und neben traditionellen Autoritäten ebenso alle anderen relevanten Akteure zu berücksichtigen.

Die Verknüpfung der unterschiedlichen Zugangsmechanismen mit einem differenzierten Verständnis von Machtverhältnissen bedeutet für die Analyse der empirischen Daten Folgendes: Zunächst muss sie untersuchen, inwiefern unterschiedliche rechtebasierte, strukturelle sowie relationale Zugangsmechanismen in den erforschten Konflikten relevant sind und zwischen welchen Akteuren sie auf welche Weise ausgehandelt werden. Darauf aufbauend ist das Ziel der Analyse, herauszuarbeiten, inwiefern sich unterschiedliche 
Machtformen in Konflikten auf die Fähigkeit Zugang zu Land zu kontrollieren, auswirken.

\subsection{Operationalisierung der theoretischen Kategorien}

Die zuvor erarbeiteten zentralen Analysekategorien für meiner Studie sind rechtebasierte, strukturelle und relationale Zugangsmechanismen sowie sichtbare, versteckte und unsichtbare Macht. Für die empirische Analyse erarbeite ich Indikatoren, anhand derer ich erkenne, dass es sich bei einem in der Forschung beobachteten Phänomen um einen Zugangsmechanismus oder eine Form von Macht handelt.

\section{Operationalisierung der Zugangsmechanismen}

Die Identifizierung der im Konflikt umkämpften Zugangsmechanismen zu Land dient der Strukturierung der Analyse und soll den Blick für mögliche Konfliktgegenstände schärfen. Welche Zugangsmechanismen in den untersuchten Konflikten um Land von Bedeutung sind, erfasse ich analytisch über die Veränderung von Zugangsbedingungen. Hinweise auf Veränderungen von Zugangsbedingungen ermittle ich über die Fragen, wie und auf welcher Grundlage Akteure vor der Aneignung des Lands durch das Unternehmen Zugang zu diesem erlangt haben und mit welchen Konsequenzen sich dies im Kontext von land grabbing verändert hat. Im Folgenden entwickle ich Indikatoren, anhand derer ich rechtebasierte, relationale und strukturelle Zugangsmechanismen in der Analyse erkennen kann.

Rechtebasierte Zugangsmechanismen sind legale Mittel, die Zugang $\mathrm{zu}$ Land über gesellschaftlich anerkannte Rechte ermöglichen, sowie illegale Mittel, die in Abgrenzung zu legalen Mitteln existieren, also nicht auf gesellschaftlicher Akzeptanz beruhen und Zugang zu Land über nichtinstitutionelle Wege ermöglichen. In traditionellen, durch rechtspluralistische Strukturen geprägten Landrechtssystemen finden gesellschaftlich anerkannte Rechte in den folgenden Formen Ausdruck: in behördlichen Besitzurkunden, schriftlich oder mündlich festgehaltenen Pachtverträgen, in Landnutzungsrechten, die traditionelle Institutionen oder individuelle Landrechteinhaber*innen vergebenen oder in sharecropping-Vereinbarungen. Auch Entschädigungszahlungen für den Verlust von Land zählen zu den rechtebasierten Zugangsmechanismen, da die Voraussetzung für ihren 
Erhalt zuvor bestehende Rechte an Land sind. Legale Mittel können den eigenen Zugang zu Land gewährleisten oder ermöglichen, Zugang zu Land an Dritte zu übertragen. Gegenleistungen für die Übertragung von Rechten in Form von Naturalien oder Pachtzahlungen führen dazu, dass beide Seiten einen Nutzen aus dem Land ziehen. Illegale Mittel umfassen die Androhung oder Anwendung von Gewalt, Diebstahl oder das Vortäuschen falscher Tatsachen. Letzteres bedeutet, dass Akteure Zugang zu Land erlangen, indem sie - obgleich dies nicht der Fall ist - behaupten, über eine Besitzurkunde oder traditionelle Rechte an Land zu verfügen. Insbesondere in Bezug auf traditionelle Rechte an Land, für die es keinen handfesten Beweis in Form einer vorzeigbaren Besitzurkunde gibt, kann es empirisch schwierig sein, eindeutig zwischen legalen oder illegalen Mitteln zu unterscheiden. Denn die Behauptungen unterschiedlicher Akteure können sich diesbezüglich widersprechen. Indikator für rechtebasierte Zugangsmechanismen ist der Einsatz legaler oder illegaler Mittel, um Zugang zu Land einzufordern. Beispiele für Indikatoren rechtebasierter Zugangsmechanismen sind, dass Akteure sich auf seit Generationen bestehende traditionelle Landrechte berufen oder Besitzurkunde vorweisen, um Zugang zu Land einzufordern. Ein weiteres Beispiel ist die Androhung von Waffengewalt, um sich Zugang zu Land zu verschaffen.

Relationale und strukturelle Zugangsmechanismen komplementieren die rechtebasierten Zugangsmechanismen, insbesondere in traditionellen Landrechtssystemen. Relevante relationale und strukturelle Zugangsmechanismen in traditionellen Landrechtssystemen sind soziale Zugehörigkeit, Netzwerke und soziale Beziehungen. Diese Zugangsmechanismen sind, im Rahmen struktureller Grenzen, verhandelbar und stehen in einem wechselseitig konstitutiven Verhältnis zu rechtebasierte Zugangsmechanismen. Soziale und institutionelle Rahmenbedingungen, die Zugang zu Land ermöglichen, sind Gegenstand sozialer Aushandlungsprozesse. Sie sind nicht einmal geschaffen und damit unveränderbar, sondern stehen in einem wechselseitigen Verhältnis zu dem Handeln von Akteuren. Soziale und institutionelle Rahmenbedingungen beeinflussen das Handeln von Akteuren, das seinerseits wiederum auf die Ausgestaltung der Bedingungen zurückwirkt. Akteure verleihen sozialen und institutionellen Rahmenbedingungen Legitimität und tragen $\mathrm{zu}$ ihrem Fortbestand bei, indem sie sie anerkennen. Oder sie verändern sie, indem sie sie umdeuten. Verhandelbarkeit als analytische Kategorie muss daher in Bezug auf alle Zugangsmechanismen mitberücksichtigt werden. Indikatoren für die Verhandelbarkeit von 
Zugangsmechanismen bestehen darin, dass Akteure existierende soziale und institutionelle Rahmenbedingungen infrage stellen oder neu auslegen, um Zugang zu Land zu erlangen oder anderen Akteuren den Zugang zu verwehren. Beispiele hierfür sind die Umdeutung oder Neuinterpretation traditioneller Landrechte zu Gunsten bestimmter Akteure sowie Bestrebungen von Akteuren, die eigene Zugehörigkeit oder diejenige anderer, neu auszulegen, mit dem Ziel, Zugang zu Land zu erlangen oder zu beschränken.

Soziale Zugehörigkeit, die mit strukturell begründeten Ungleichheitsverhältnissen einhergeht, beeinflusst die Möglichkeiten, Zugang zu Land - insbesondere rechtebasierten Zugang - zu erlangen. Indigene Landnutzer*innen - also diejenigen, die vorgeben schon immer in der Gegend gelebt $\mathrm{zu}$ haben oder die Ersten gewesen zu sein, die das Land bestellt haben - verfügen in der Regel über permanente Rechte an Land. Je nach Kontext können diese Rechte für Mitglieder der Gemeinschaft übertragbar sein. Teilweise beschränkt sich dieses Recht auf Männer. Zugezogene Landnutzer*innen aus anderen Staaten oder Gegenden erhalten in der Regel nur temporäre Rechte. Für indigene Landnutzer*innen bleiben Rechte an Land auch bestehen, wenn sie es eine Weile brach liegen lassen. Je nach sozialer Zugehörigkeit sind für manche Landnutzer*innen Rechte zudem mit Pflichten verbunden. Der Erhalt von Weide- oder Anbaurechten kann mit der Abgabe landwirtschaftlicher Erzeugnisse einhergehen (Shipton/Goheen 1992: 311). Indikator für soziale Zugehörigkeit als relevanter Mechanismus für Zugang zu Land ist die Einforderung, Legitimierung oder Beschränkung von Zugang zu Land über Kategorien sozialer Zugehörigkeit wie Indigenität, first comer/late comer, Herkunft, Geschlecht oder Alter. Auch Wissen spielt eine wichtige Rolle in Bezug auf Zugang zu Land. Wissen findet Ausdruck in formeller Bildung, in Kenntnissen der eigenen Rechte oder Kenntnissen kontextspezifischer Informationen. Diese können Akteure dazu befähigen, Zugang zu Land zu erlangen oder aufrechtzuhalten. Die Fähigkeit zu lesen, ist die Voraussetzung dafür, schriftliche Informationen, wie Ankündigungen zu einem geplanten Projekt, zu erhalten. Die Fähigkeit zu schreiben ist notwendig, um formelle Briefe oder Anträge verfassen zu können. Kenntnisse rechtlicher Rahmenbedingungen sind notwendig für die Aushandlung von Zugang zu Land, z.B. über die Neuinterpretation bestehender Regeln und Normen. Fachkenntnisse können eine Voraussetzung dafür sein, bestimmte Arbeiten, wie das Führen von Maschinen, leisten zu können. Darüber hinaus sind Kenntnisse kontextspezifischer Informationen zentral, um überhaupt auf einen möglichen eigenen Nachteil zu reagieren. Akteure die nichts über ein geplantes Projekt wissen, 
können auch die damit verbundenen negativen Auswirkungen für sich nicht realisieren und werden dementsprechend keine Gegenmaßnahmen ergreifen oder die Teilnahme an Entscheidungsprozessen einfordern. Indikatoren für Wissen als relevanten Zugangsmechanismus ist der Rückgriff von Akteuren auf spezifische Kenntnisse, um Zugang zu Land zu erlangen oder Verweise darauf, dass sie ihren Zugang zu Land aufgrund fehlender Informationen oder Kenntnisse verloren haben. Ein Beispiel, das den Rückgriff auf spezifische Kenntnisse veranschaulicht, sind Bestrebungen von Akteuren, Zugang zu Land über andere als die bisher genutzten Wege - z.B. über Gerichtsverfahren - zu erlangen. Aussagen von Akteuren zur Intransparenz der Landvergabe und dem daraus resultierenden Verlust des Lands können ein Hinweis auf den Indikator der fehlenden Informationen sein.

Soziale Zugehörigkeit ist darüber hinaus auch für die anderen relationalen und strukturellen Zugangsmechanismen - Netzwerke und soziale Beziehungen - bedeutsam. Die mit sozialer Zugehörigkeit verbundenen Handlungsmöglichkeiten und -grenzen - beeinflussen, welche sozialen Beziehungen Akteure eingehen und in welchen Netzwerken sie sich engagieren (können).

Netzwerke als relationaler und struktureller Mechanismus können auf vielfältige Weise relevant für den Zugang zu Land sein: Netzwerke ermöglichen es, gemeinsam Einfluss auf Debatten und Entscheidungsprozesse zu nehmen, Wissen zu teilen und sich gegenseitig zu unterstützen. Indikator für Netzwerke als Zugangsmechanismus ist, dass die Zugehörigkeit zu Netzwerken Auswirkungen auf den Zugang zu Land hat. Die Zugehörigkeit zu traditionellen Institutionen und damit verbundene Privilegien in Bezug auf Zugang zu Land ist ein Beispiel für diesen Indikator. Ein weiteres Beispiel ist die Mitgliedschaft in einem professionellen Netzwerk, das über spezifisches Wissen Zugang zu Land ermöglichen kann. Auch in Bezug auf Zugang zu Land über Arbeit spielen Netzwerke eine zentrale Rolle. Über die Mitgliedschaft in Netzwerken erlangen Akteure möglicherweise privilegierten Zugang $\mathrm{zu}$ Arbeit.

Der dritte relationale und strukturelle Zugangsmechanismus sind soziale Beziehungen. Indikator für diesen Zugangsmechanismus ist, dass Akteure Zugang zu Land maßgeblich über Beziehungen zu anderen Akteuren erlangen. Ein Beispiel ist die Möglichkeit migrantischer Landnutzer*innen Zugang zu Land zu erlangen, indem sie sich um eine gute Integration in die lokale Gemeinschaft bemühen. Ein weiteres Beispiel ist der Zusammenhang von Zugang zu Land über Arbeit und einer guten Beziehung zu 
Akteuren, die diesbezüglich eine gate keeper-Funktion einnehmen. Auch sharecropping-Vereinbarungen oder andere individuelle Vereinbarungen, die Zugang zu Land ermöglichen, sind Indikatoren für soziale Beziehungen als Zugangsmechanismus. Zugang zu Land über sharecropping veranschaulicht, wie sich relationale und strukturelle Zugangsmechanismen mit rechtebasierten Zugangsmechanismen überschneiden.

\section{Operationalisierung der sichtbaren, versteckten und unsichtbaren Macht}

In der Analyse meiner beiden Fälle untersuche ich, inwiefern sich sichtbare, versteckte und unsichtbare Machtverhältnisse zwischen den Konfliktakteuren auf die Kontrolle ihres Zugangs zu Land - operationalisiert mittels der unterschiedlichen Zugangsmechanismen - auswirken und inwiefern sich die Ausübung unterschiedlicher Machtformen auf die Entstehung und den Verlauf von Konflikten um Zugang zu Land auswirken. Zu diesem Zweck entwickle ich im Folgenden Indikatoren, anhand derer ich in der Analyse erkenne, dass es sich um eine der drei Machtformen handelt.

Die Analyse von Machtverhältnissen beschäftigt sich im Kern mit der Frage, wie es Akteuren gelingt, sich die Zustimmung von Gegenspieler*innen zu sichern. Um von Machtausübung sprechen zu können, müssen für die Analyse zwei zentrale Bedingungen erfüllt sein. Die erste Bedingung ist, dass eine Person (A) auf eine bestimmte Weise handelt oder bewusst nicht handelt. Die zweite Bedingung ist, dass aufgrund dieses Handelns eine zweite Person (B) etwas tut, das sie sonst nicht tun würde. »Tun« bezieht sich auf ein breites Verständnis, das auch »denken«, »wollen«, »akzeptieren« oder »fühlen« umfasst. A kann B allein durch ihr Handeln oder im Zusammenspiel mit anderen Faktoren dazu bringen, etwas zu tun, das B sonst nicht tun würde (Lukes 2005: 43). Damit wir von Machtausübung sprechen können, bedarf es immer der Möglichkeit eines kontrafaktischen Szenarios, also der Option, dass B sich in Abwesenheit des Handelns von A anders entscheiden würde.

Der zentrale Indikator für die Ausübung sichtbarer Macht ist ein Befehl, der Gehorsam findet. Ein »Befehl« kann eine Anweisung oder eine Entscheidung sein, die Konsequenzen nach sich zieht. »Gehorsam finden« kann bedeuten, dass Gegenspieler*innen nicht in der Lage sind, sich dem Befehl zu widersetzen, dass sie ihn widerstandslos akzeptieren oder sogar unterstützen, auch wenn er sich gegen ihre Interessen richtet. Ein Beispiel ist die Fähigkeit von Akteuren, alleinige Kontrolle über den Zugang zu Land aus- 
zuüben. Das bedeutet, diese können eigenständig die Entscheidung treffen, Land zu vergeben, auch gegen den Willen anderer Akteure, die nicht die Fähigkeit haben, sie daran zu hindern.

Versteckte Macht umfasst alle Formen der Kontrolle von A über B, mittels derer A sich die Zustimmung von B sichert. In Konflikten um Zugang bezieht sich die Ausübung versteckter Macht also auf die Kontrolle des Zugangs zu Land. Formen der Kontrolle sind vielfältig. Sie umfassen die Kontrolle von Informationen, den Einfluss auf institutionelle Verfahrensweisen und Partizipationsmöglichkeiten, aber auch die Androhung negativer Konsequenzen oder Gewalt. Ein zentraler Indikator für die Ausübung versteckter Macht ist Ausschluss. Ausschluss kann bedeuten, dass A entscheidet, Themen, die ihren eigenen Interessen schaden könnten, jedoch für B von Bedeutung sind, von der Agenda auszuschließen und nicht zu einem potentiellen Aushandlungsgegenstand zu machen. Das bedeutet B hat die Möglichkeit über x zu verhandeln, nicht aber über $y$. Darüber hinaus kann sich Ausschluss darauf beziehen, dass das Handeln von A die Möglichkeiten von B, Entscheidungen zu treffen, einschränkt. Wenn B beispielsweise keine Informationen darüber erhält, dass eine bestimmte Entscheidung zur Verhandlung steht, hat sie auch nicht die Möglichkeit an diesem Entscheidungsfindungsprozess teilzuhaben. Um diese Form der versteckten Macht zu identifizieren, ist es sinnvoll, die Ausgestaltung institutioneller Verfahrensweisen und die damit verbundenen Handlungsmöglichkeiten der Akteure in den Blick zu nehmen. Formen der Kontrolle können darüber hinaus Zwang, Einfluss, Autorität und Manipulation umfassen (Lukes 2005: 21). Der Indikator für die Ausübung versteckter Macht mittels Zwangs ist die Zustimmung aus Angst vor negativen Konsequenzen. Ein Beispiel dafür ist, dass A ihre eigenen Interessen entgegen derjenigen von B durchsetzen kann, indem sie B bei Widerstand mit Konsequenzen droht, die im Gegensatz zu ihren Interessen stehen. Der Indikator für die Ausübung versteckter Macht mittels Einflusses ist die Zustimmung von B aufgrund positiver Erwartungen. A übt Einfluss auf B aus, wenn sie sich den bereitwilligen Gehorsam von B sichert, ohne dafür implizit oder explizit negative Konsequenzen androhen zu müssen. Dies kann A beispielsweise gelingen, indem sie mögliche positive Auswirkungen der Entscheidung in den Mittelpunkt rückt oder B »Belohnung« für ihre Zustimmung in Aussicht stellt. Der Indikator für die Ausübung versteckter Macht mittels Autorität ist Anerkennung. A übt Autorität aus, wenn B ihr Handeln widerstandslos anerkennt, da sie es als angemessen hinsichtlich ihrer eigenen verinnerlichten Werte und Normen ansieht. Dies drückt sich beispielsweise anhand von Sätzen wie »Das 
war schon immer so« oder »So funktioniert das bei uns« aus. Indikator für die Ausübung versteckter Macht mittels Manipulation ist Zustimmung aufgrund fehlender Informationen. Ein Beispiel dafür ist ein Hinweis darauf, dass A in Bezug auf eine bestimmte Entscheidung B zentrale Informationen vorenthalten hat und B aufgrund dessen der Entscheidung zustimmt. In einem Szenario, in dem Landnutzer*innen ihr Land widerstandslos einem Unternehmen überlassen, in der Annahme, angemessen dafür entschädigt zu werden, ist es wahrscheinlich, dass sie anders gehandelt hätten, wenn sie über die unangemessene Höhe der Entschädigung informiert worden wären.

Die Ausübung unsichtbarer Macht bedeutet, andere so in ihren Einstellungen und Bedürfnissen zu beeinflussen, dass sie - indem sie zustimmen, einwilligen oder keinen Widerstand leisten - letztendlich entgegen ihrer ethischen Interessen handeln und willig an ihrer Unterdrückung mitwirken. Die Herausforderung, diese Form der Machtausübung empirisch zu analysieren, liegt darin, die ethischen Interessen der Akteure zu ermitteln.

Die Bestimmung von Interessen - sofern es sich nicht um manifeste Interessen handelt, die Akteure formulieren und/oder die sich aus ihren Handlungen ablesen lassen - ist grundsätzlich schwierig. Akteure haben in der Regel vielfältige unterschiedliche, teilweise widersprüchliche oder nicht miteinander vereinbare Interessen (Lukes 2005: 147). So können Interessen bezüglich des eigenen psychischen Wohlbefindens solchen gegenüberstehen, die sich auf das finanzielle Wohlergehen und die Sicherung eigener Grundbedürfnisse beziehen. Eine Person kann das Bedürfnis haben, im Sinne des psychischen Wohlbefindens mehr Erholungszeit für sich zu haben, bemüht sich aber dennoch um eine neue Arbeit mit höherem Stundenumfang, da sie ebenfalls ein Interesse daran hat, mehr zu verdienen. Wie ist es also möglich, empirisch die ethischen Interessen von Akteuren zu ermitteln, ohne a priori gesetzte Annahmen zu treffen und aus einer Außenperspektive den Akteuren Interessen zu unterstellen? Und wenn wir annehmen, dass Akteure vielfältige, auch widersprüchliche Interessen haben, welche dieser unterschiedlichen Interessen bestimmen wir dann letztendlich als die ethischen Interessen?

Die Außenperspektive der Forschenden in der Untersuchung unsichtbarer Machtverhältnisse ist in dem Konzept der unsichtbaren Macht selbst verankert. Die Annahme, dass die Zustimmung schwächerer Akteure zu ihrer Unterdrückung über die Einflussnahme auf ihre Einstellungen und Bedürfnisse sichergestellt wird, erfordert ein Urteilsvermögen, das sich von dem der vermeintlich betroffenen Akteure unterscheidet (Lukes 2005: 146). Denn die 
Ausübung unsichtbarer Macht beruht auf der Fähigkeit von A zu täuschen, und damit bei B falsche Annahmen zu erzeugen (ebd. : 149).

Für die Ermittlung ethischer Interessen bedarf es eines Referenzrahmens, der bestimmt, auf welchen Gegenstand sich die Untersuchung bezieht. Diesen Referenzrahmen setzt das Forschungsthema. Welche Interessen als ethische Interessen im Zentrum der Analyse stehen sollen und in welches Verhältnis unterschiedliche Interessen zueinander gesetzt werden, ergibt sich demnach aus der Perspektive der Forschenden. Sie setzt den Schwerpunkt der Untersuchung. In dieser Studie sind die ethischen Interessen von Akteuren in Bezug auf den Zugang zu Land zu ermitteln. Diesbezüglich stellt sich die Frage, inwiefern der Verlust von Zugang zu Land den Voraussetzungen für ein gutes Leben und somit den ethischen Interessen entgegensteht? Für die Operationalisierung ethischer Interessen nehme ich eine menschenrechtliche Perspektive ein. Insbesondere beziehe ich mich auf den Pakt über wirtschaftliche, soziale und kulturelle Rechte (United Nations General Assembly 1966). Demnach sind grundlegende Voraussetzungen für ein gutes Leben das Recht auf angemessene Entlohnung, auf angemessenen Lebensstandard, der sich über angemessene Nahrung, Kleidung und Wohnung ausdrückt, sowie das Recht auf Bildung. Diesbezüglich geht es mir nicht um eine normative Beurteilung, was als angemessen angesehen werden kann. Vielmehr gehe ich davon aus, dass die ethischen Interessen von Akteuren dann verletzt werden, wenn sich ihre Situation in Bezug auf diese Rechte im Verlauf der Landvergabe verschlechtert hat. Wenn eine Landnutzerin beschreibt, dass land grabbing dazu geführt hat, dass ihr Einkommen - im Gegensatz zu vorher - nicht mehr ausreicht, um genügend Lebensmittel zu kaufen oder ihren Kindern eine angemessene Bildung zu ermöglichen, deutet dies darauf hin, dass gegen ihre ethischen Interessen gehandelt wurde.

Aus Perspektive des Konzepts der unsichtbaren Macht beeinflusst die gesellschaftliche Ordnung die Bedürfnisse und Einstellungen von Akteuren und wirkt damit potenziell gegen deren ethische Interessen. Ein Ansatzpunkt, um die ethischen Interessen von Akteuren zu ermitteln, besteht darin $\mathrm{zu}$ bestimmen, was Akteure tun oder bevorzugen würden, wenn sie frei von gesellschaftlichen, strukturellen und wirtschaftlichen Zwängen entscheiden könnten (kontrafaktisches Handeln) (Lukes 2005: 38). Ethische Interessen sind nicht als »Tatsachen« zu denken, sondern als analytisches Hilfsmittel, um ein erklärendes Verstehen in einem bestimmten Sinnzusammenhang zu ermöglichen. Ihre Bestimmung dient nicht dazu, eine »Wahrheit« aufzudecken, sondern dazu zu zeigen, dass Akteure Interessen haben (können), 
für die sie nicht eintreten (können oder wollen), da sich ihnen aufgrund der gesellschaftlichen Ordnung, in der sie leben, oder aufgrund ihrer Sozialisation keine entsprechenden Handlungsmöglichkeiten bieten. Sie verhandeln daher nur Themen, die es bereits in Entscheidungsräume geschafft haben oder bezüglich derer sie Handlungsmöglichkeiten erkennen. Das bedeutet jedoch nicht, dass sich Akteure keiner anderen Interessen bewusst werden können, wenn sie sich beispielsweise in ein kontrafaktisches Szenario hineindenken oder eine Zukunftsvision entwickeln. Es bedeutet nur, dass sie ohne solche Vorstellungen bestimmte Interessen möglicherweise nicht formulieren, da sie sie als utopisch, unrealistisch und somit nicht handlungsrelevant erachten. Über die Bestimmung kontrafaktischen Handelns lassen sich so tieferliegende Interessen identifizieren. Denn Akteure kennen die Handlungsmöglichkeiten und -beschränkungen sowie die Konsequenzen von Handlungen, die mit bestimmten sozialen Strukturen verbunden sind. Abhängig von ihrer sozialen Position leiten sie daraus Gründe für ihr Handeln oder Nicht-Handeln ab und formulieren ihre Interessen entsprechend (Schuck 2014: 316). Indem Akteure sich also vorstellen, von einzelnen »Zwängen« befreit zu sein, können sie sich ihren tieferliegenden Interessen annähern. Darüber hinaus gilt zu berücksichtigen, dass gesellschaftliche Strukturen oder Sozialisation Einstellungen und Bedürfnisse eventuell bereits so beeinflusst haben, dass Akteure bestimmte Handlungsmöglichkeiten gar nicht in Erwägung ziehen.

In Konflikten um Zugang bedeutet die Ausübung unsichtbarer Macht, dass machtvolle Akteure Zugang zu Land beschränken können, ohne dass diejenigen, deren Zugang beschränkt wird, Widerstand leisten. Der fehlende Widerstand kann darin begründet liegen, dass Akteure keine Alternative sehen oder dass sie fälschlicherweise annehmen, die Entscheidung könne vorteilhaft für sie sein. Ein Indikator für die Ausübung versteckter Macht ist die Übernahme zentraler Argumente von stärkeren Akteuren durch die schwächeren Akteure und daraus resultierendes Handeln oder Nicht-Handeln entgegen der ethischen Interessen. Dies kann auf dominante Diskurse oder Sozialisationsprozesse zurückzuführen sein. Ein weiterer Indikator sind Hinweise auf Täuschung oder das Vortäuschen falscher Tatsachen. Ein Beispiel dafür könnte sein, dass Akteure eine bestimmte Entscheidung aufgrund vermeintlicher Vorteile für gut halten, diese Vorteile ihnen selbst jedoch nachweislich nicht zugutekommen. 
Tabelle 2: Operationalisierung der theoretischen Kategorien

\begin{tabular}{|c|c|}
\hline Kategorie & Indikatoren \\
\hline $\begin{array}{l}\text { Rechtebasierte } \\
\text { Zugangsmechanismen }\end{array}$ & $\begin{array}{l}\text { Akteure setzen legale und illegale Mittel ein, um Zugang zu } \\
\text { Land zu erlangen } \\
\text { Beispiele für legale Mittel: Besitzurkunden, Pachtverträge, } \\
\text { traditionelle Rechte, sharecropping-Vereinbarungen, Entschädi- } \\
\text { gungszahlungen etc. } \\
\text { Beispiele für illegale Mittel: Androhung oder Anwendung von } \\
\text { Cewalt, Diebstahl, Vortäuschen falscher Tatsachen etc. }\end{array}$ \\
\hline $\begin{array}{l}\text { Relationale und } \\
\text { strukturelle } \\
\text { Zugangsmechanismen }\end{array}$ & $\begin{array}{l}\text { Akteure beziehen sich auf ihre soziale Zugehörigkeit, um Zu- } \\
\text { gang zu Land zu erlangen } \\
\text { Beispiele für Kategorien sozialer Zugehörigkeit: Autochthonie, } \\
\text { first comer/late comer, Herkunft, Geschlecht, Alter }\end{array}$ \\
\hline
\end{tabular}

Akteure interpretieren Landrechte zu ihren Gunsten

Beispiel: Akteure verhandeln ihre soziale Zugehörigkeit, um Zugang zu Land zu erlangen.

Akteure erlangen Zugang zu Land über die Mitgliedschaft in Netzwerken

Beispiele für Netzwerke: lokale Gemeinschaft, traditionelle Institutionen, professionelle Netzwerke, politische Netzwerke

Akteure erlangen oder kontrollieren Zugang zu Land aufgrund spezifischer Kenntnisse

Beispiele für spezifische Kenntnisse: Formelle Bildung, Kenntnisse der eigenen Rechte, Kenntnisse kontextspezifischer Informationen

Akteure erlangen Zugang zu Land aufgrund sozialer Beziehungen

Beispiele: Beziehung zu Personen, die Zugang zu Land kontrollieren, Integration von Migrant"innen in lokaler Cemeinschaft, Zugang zu Arbeit über Beziehungen etc. 


\begin{tabular}{|c|c|}
\hline Kategorie & Indikatoren \\
\hline Sichtbare Macht & $\begin{array}{l}\text { Befehl findet Gehorsam } \\
\text { Beispiel: A bringt B dazu etwas zu tun (denken, fühlen, wollen, } \\
\text { akzeptieren), auch wenn B etwas anderes vorziehen würde. }\end{array}$ \\
\hline Versteckte Macht & $\begin{array}{l}\text { Ausschluss von Akteuren oder Themen } \\
\text { Beispiele: } \\
\text { a)Institutionelle Verfahrensweisen verhindern die Herausforde- } \\
\text { rung etablierter Werte und Normen } \\
\text { b) Beschränkung der Verhandlung widersprüchlicher Interessen } \\
\text { durch den Ausschluss bestimmter Themen aus Entscheidungs- } \\
\text { räumen (Nicht-Entscheidungen) } \\
\text { c)Beschränkung von Entscheidungen durch Kontrolle von Infor- } \\
\text { mationen }\end{array}$ \\
\hline
\end{tabular}

Zustimmung aus Angst vor negativen Konsequenzen

Beispiel: B stimmt As Entscheidung zu, da sie aufgrund von As Androhung Angst hat.

Zustimmung aufgrund positiver Erwartungen

Beispiel: A verspricht $B$ für ihre Einwilligung eine Belohnung.

Zustimmung aufgrund fehlender Informationen

Beispiel: Bstimmt Entscheidung $x z u$, da sie Entscheidungy nicht als Alternative erkennt. 


\begin{tabular}{|c|c|}
\hline Kategorie & Indikatoren \\
\hline \multirow[t]{6}{*}{ Unsichtbare Macht } & $\begin{array}{l}\text { Schwächere Akteure übernehmen die Argumente der stärke- } \\
\text { ren Akteure }\end{array}$ \\
\hline & $\begin{array}{l}\text { Beispiel: A beeinflusst Bs Einstellungen und Absichten über die } \\
\text { Kontrolle von Informationen, Sozialisation (verinnerlichte Re- } \\
\text { geln und Normen etc.) und den Einfluss auf Diskurse. }\end{array}$ \\
\hline & Hinweise auf Täuschung \\
\hline & $\begin{array}{l}\text { Beispiel: B erklärt eine bestimmte Entscheidung aufgrund er- } \\
\text { warteter Vorteile, die ihm selbst jedoch nachweislich nicht zugu- } \\
\text { tekommen. }\end{array}$ \\
\hline & Anerkennung entgegen der eigenen Interessen \\
\hline & $\begin{array}{l}\text { Beispiel: B stimmt As Entscheidung entgegen ihrer Interessen } \\
\text { zu, da sie diese mit Blick auf verinnerlichte Werte und Normen } \\
\text { (Sozialisation) als richtig empfindet. }\end{array}$ \\
\hline
\end{tabular}





\section{Forschungsdesign und methodisches Vorgehen}

Das Forschungsdesign meiner Studie leitet sich aus dem Ziel ab, empirisch begründetes Wissen zum Zusammenhang des Handelns traditioneller Autoritäten und Konflikten um Land im Kontext von land grabbing zu schaffen. Dazu analysiere ich zwei Konflikte um die großflächige agrarindustrielle Umnutzung von Land in der Ashanti Region und Northern Region Ghanas. In beiden Fällen sind traditionelle Autoritäten als zentrale Akteure in die Konflikte involviert. Auf welche Weise traditionelle Autoritäten handeln und welche Rolle sie in Konflikten, die im Zusammenhang mit land grabbing auftreten, einnehmen, ist immer kontextabhängig und damit unter Berücksichtigung der jeweiligen Bedingungen zu analysieren. Dennoch erlauben die Erkenntnisse meiner Forschung es, Schlussfolgerungen zu ziehen, deren Reichweite über die untersuchten Fälle und den Länderkontext hinausgeht. Dazu tragen das vergleichende Forschungsdesign sowie Parallelen in der traditionellen Herrschaft unterschiedlicher postkolonialer Staaten bei, die auf ihre gemeinsame Kolonialvergangenheit zurückzuführen sind.

Im Folgenden stelle ich zunächst das Forschungsdesign meiner Studie vor, lege die Kriterien der Fallauswahl dar und gehe auf den Vergleich der beiden Fälle ein. Daran anschließend erläutere ich die für die Datenerhebung angewandten Methoden sowie meine Vorgehensweise bei der Datenauswertung.

\subsection{Kollektive Fallstudie}

Meine Forschung ist als eine Fallstudie mit instrumenteller Ausrichtung konzipiert (Stake 1995; Stake 2003). Eine Fallstudie ist die ausführliche empirische Untersuchung eines Phänomens unter Berücksichtigung des jeweiligen lebensweltlichen Kontexts (Yin 2009: 18). Ein Fall ist eine räumlich begrenz- 
te Einheit, die zu einem bestimmten Zeitpunkt oder über einen begrenzten Zeitraum hinweg erforscht wird (Gerring 2007: 19). In einer instrumentellen Fallstudie dient der Fall dazu, ein allgemeines Verständnis für ein spezifisches Phänomen zu entwickeln. Instrumentelle Fallstudien eignen sich insbesondere, um Hypothesen hinsichtlich eines bisher wenig erforschten Phänomens $\mathrm{zu}$ entwickeln. Je nach Erkenntnisinteresse kann es - wie auch für meine Studie - sinnvoll sein, mehr als nur einen einzigen Fall zu erforschen. Die Ausweitung instrumenteller Fallstudien auf mehrere Fälle bezeichnet Robert Stake (2003: 138) als kollektive Fallstudie.

Ein wichtiger Ausgangspunkt für die Analyse des Handelns traditioneller Autoritäten in Konflikten um Land ist die unterschiedliche Ausgestaltung traditioneller Herrschaft. Daher vergleiche ich zwei Fälle, in denen sich das traditionelle Herrschaftssystem unterscheidet. Ein Paarvergleich, (paired comparison) (Tarrow 2010) - wie ich ihn in meiner Studie durchführe - bietet im Verhältnis zu einer Einzelfallstudie eine ausgewogene Kombination aus deskriptiver Tiefe und analytischer Herausforderung. Der Schritt von einem Einzelfall hin $\mathrm{zu}$ einem Paarvergleich erlaubt es, verallgemeinerbare Hypothesen $\mathrm{zu}$ generieren und gleichzeitig in der Lage zu sein, zu überprüfen wie unterschiedliche Variablen interagieren.

\subsection{Kriterien der Fallauswahl}

In der Logik meiner Studie ist ein Fall ein Konflikt um Land im Kontext von land grabbing, in dem traditionelle Autoritäten zu den zentralen Konfliktakteuren zählen. Land grabbing verstehe ich in Anlehnung an Borras et al. (2012: 405) als die Aneignung von Kontrolle über relativ große Flächen Land und andere Ressourcen, die mit einer intendierten Inwertsetzung einhergeht und eine Verlagerung weg von der Ressourcennutzung hin zur Ressourcengewinnung für den heimischen oder globalen Markt mit sich bringt. Um den Fall zeitlich und räumlich einzugrenzen, eignen sich empirisch beobachtbare Konflikte, die im Zusammenhang mit der großflächigen Umnutzung von Land im Rahmen agrarindustrieller Projekte auftreten. Empirisch beobachtbare Konflikte verstehe ich als soziale Beziehungen zwischen mindestens zwei individuellen oder kollektiven Akteuren, gekennzeichnet durch von ihnen als widersprüchlich wahrgenommene Interessen, Ziele oder Bedürfnisse (Bonacker/Imbusch 2006), die in Handeln resultieren (Dietz/Engels 2014). 
Für die Fallauswahl setzen diese Kriterien voraus, dass es sich um einen Länderkontext handelt, in dem land grabbing relevant ist und traditionelle Autoritäten im Vergleich zu staatlichen Institutionen eine starke Stellung in der Verwaltung und Vergabe von Land sowie in der Regelung von Konflikten einnehmen. Aufgrund der theoretischen Vorannahme, dass die unterschiedliche Ausgestaltung traditioneller Herrschaft relevant für das Handeln traditioneller Autoritäten in Konflikten sein könnte, beziehe ich mich in meinem Vergleichsdesign auf die diverse-case-Strategie nach John Gerring (2007: 97). In diesem Fallstudiendesign zeichnen sich die einzelnen Fälle dadurch aus, dass sie sich hinsichtlich eines Aspekts oder einer Variable unterscheiden, während sie ansonsten eine gute Vergleichbarkeit charakterisiert. Eine Varianz bezüglich zentraler Aspekte des theoriegeleiteten Erkenntnisinteresses erhöht die Möglichkeit, Erkenntnisse und Schlussfolgerungen auf andere Kontexte übertragen zu können (Gerring 2007: 97f.). Daraus ergibt sich ein weiteres Kriterium für die Fallauswahl: die unterschiedliche Ausgestaltung der traditionellen Herrschaft. Für eine gute Vergleichbarkeit sollten sich andere intervenierende Kontextfaktoren durch eine größtmögliche Ähnlichkeit auszeichnen. Der Vergleich innerhalb desselben Länderkontexts ermöglicht es, die nationalen politischen und ökonomischen Kontextfaktoren stabil zu halten, die in der Literatur als relevant in Bezug auf Konflikte um Land im Kontext von land grabbing gelten (Hall et al. 2015). Darüber hinaus habe ich bei der Fallauswahl auf eine gute Vergleichbarkeit hinsichtlich des Zeitpunkts der Landvergabe, des Ausmaßes der gepachteten Fläche, des Zwecks der Umnutzung des Landes und der vorherigen Landnutzung durch die Landnutzer*innen geachtet.

\section{Auswahl des Fallstudienlands}

Vier Faktoren waren ausschlaggebend für die Auswahl des Fallstudienlands. Erstens, eine hohe Relevanz traditioneller Autoritäten in den Bereichen Landverwaltung und Konfliktmanagement. Zweitens, die deutlich unterschiedliche Ausgestaltung traditioneller Herrschaft innerhalb des gleichen Länderkontexts. Drittens, ein zahlen- und flächenmäßig großes Ausmaß von land grabbing. Und viertens, die Existenz von Konflikten um Land im Kontext von land grabbing in denen traditionelle Autoritäten als Konfliktakteure in Erscheinung treten.

Der aktuelle Stand der Forschung zeigt, dass traditionelle Autoritäten in vielen Ländern Sub-Sahara Afrikas eine bedeutende Rolle in der Landverwal- 
tung einnehmen (Stacey 2015: 26). Die staatliche Anerkennung traditioneller Autoritäten als Verwalter*innen von Land variiert in den unterschiedlichen Ländern erheblich. In vielen Ländern Subsahara-Afrikas üben sie diese Aufgabe in der alltäglichen Praxis aus, ohne eine rechtliche Grundlage und ohne offizielle Anerkennung oder Legitimierung durch den Staat. Ausnahmen diesbezüglich bilden unter anderem Ghana, Mosambik und Südafrika (Ubink 2008c), wo postkoloniale Regierungen die Stellung traditioneller Autoritäten $\mathrm{ab}$ den frühen 1980er Jahren formalisiert haben und traditionelle Herrschaft durch die verfassungsrechtliche Anerkennung staatlich legitimiert ist. In Ghana ist die formale Anerkennung traditioneller Herrschaft durch die Verfassung von 1992 gewährleistet (vgl. Kasanga/Kotey 2001). Ghana und Südafrika heben sich von anderen Ländern Subsahara-Afrikas insbesondere dadurch ab, dass traditionelle Autoritäten einen großen Einfluss in öffentlichen Angelegenheiten haben.

Die Relevanz traditioneller Autoritäten in den Bereichen Landverwaltung und Konfliktmanagement ergibt sich nicht alleine aus der staatlichen Anerkennung und Formalisierung traditioneller Herrschaft, sondern auch aus der Ausgestaltung der alltäglichen Praxis. Im Unterschied zu Ländern wie Mosambik und Südafrika, genießen traditionelle Autoritäten in Ghana neben der formalen Anerkennung auch eine besondere Anerkennung und Akzeptanz seitens der Bevölkerung, wie Umfragen des Afrobarometers zeigen (Logan 2013). Sowohl im Bereich der Landvergabe wie auch des Konfliktmanagements ist ihr Einfluss besonders groß. Die Umfrageergebnisse zeigen, dass traditionelle Autoritäten in der Landvergabe und im Konfliktmanagement nach Wahrnehmung der Befragten die Hauptverantwortung tragen. Die Studie berücksichtigt 18 Länder. Von diesen ist Ghana das einzige Land, das sich in beiden Bereichen unter den ersten drei Ländern mit den höchsten Umfragewerten für die Zuständigkeit traditioneller Autoritäten befindet. Die Befragten gaben an, dass traditionellen Autoritäten in Ghana im Vergleich zu staatlichen Akteuren in der Landvergabe sowie im Konfliktmanagement den größten Einfluss ausüben. Im Bereich der Landvergabe schrieben die Befragten traditionellen Autoritäten 70 Prozent der Zuständigkeit zu. Damit befindet sich Ghana an der Spitze aller Umfrageländer (Logan 2013: 361). Die folgenden Grafiken veranschaulichen wie die Öffentlichkeit die Zuständigkeit traditioneller Autoritäten in der Lösung von Konflikten (Abb. 1) und der Vergabe von Land (Abb. 2) in Ghana und 17 anderen Ländern Subsahara-Afrikas wahrnimmt. 
Abbildung 1: Wahrgenommene Zuständigkeitsverteilung zwischen Lokalregierung und traditionellen Autoritäten in der Konfliktlösung

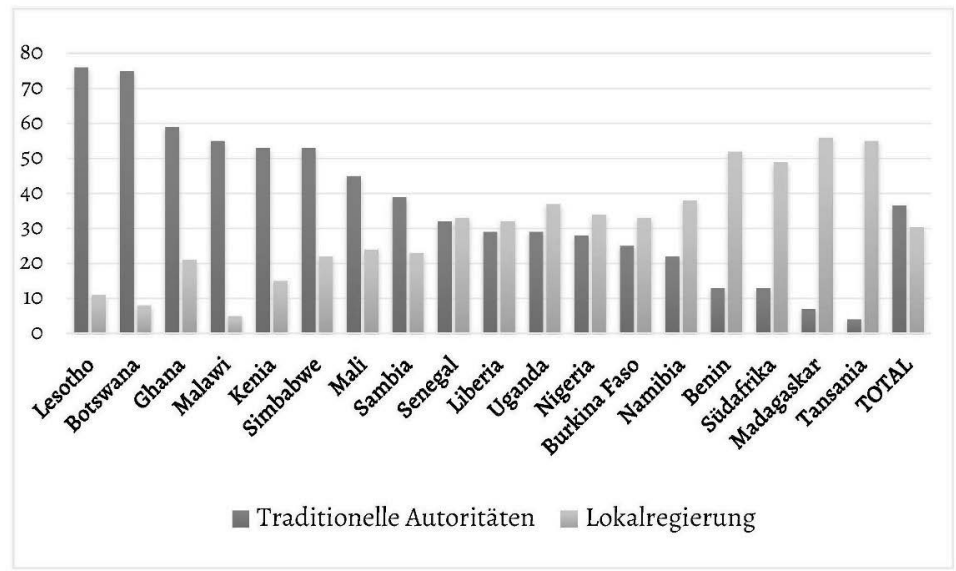

Quelle: Logan 2013: 361, eigene Darstellung, Angaben in Prozent

Gleichzeitig hat sich Ghana seit Beginn der 2000er Jahre zu einem beliebten Zielland für die Umsetzung großflächiger inter- und multinationaler agrarindustrieller Projekte entwickelt (Schoneveld/German 2014). Seit langer Zeit gilt das Land als politisch stabil, verfügt über eine vergleichsweise gute Infrastruktur und weist mit dem höchsten Bruttoinlandsprodukt pro Kopf in Westafrika eine relativ solide Wirtschaft auf. Der Agrarsektor erwirtschaftet ca. 20 Prozent des gesamten BIPs und beschäftigt die Hälfte aller Erwerbstätigen (The World Factbook 2016). Zudem hat Ghana durch eine lange Geschichte des Goldbergbaus und der Plantagenwirtschaft Erfahrungen mit internationalen Investoren. Laut Weltbank ist Ghana aufgrund der stabilen politischen Lage, günstiger steuerlicher Rahmenbedingungen und einer hohen Rechtssicherheit eines der investorenfreundlichsten Länder Afrikas (FIAN 2010: 1; Schoneveld/German 2014: 188). Zudem verfügt das Land über große Vorkommen an Rohstoffen (unter anderem Gold, Diamanten und Öl) sowie über fruchtbares Land (Annan et al. 2013: 1). Unter Berücksichtigung aller Landtransaktionen mit einer Fläche von mehr als 200 Hektar haben Investoren in Ghana, laut Land Matrix, im Zeitraum von 2000 bis 2015 Pachtverträge 
Abbildung 2: Wahrgenommene Zuständigkeitsverteilung zwischen Lokalregierung und traditionellen Autoritäten in der Landvergabe

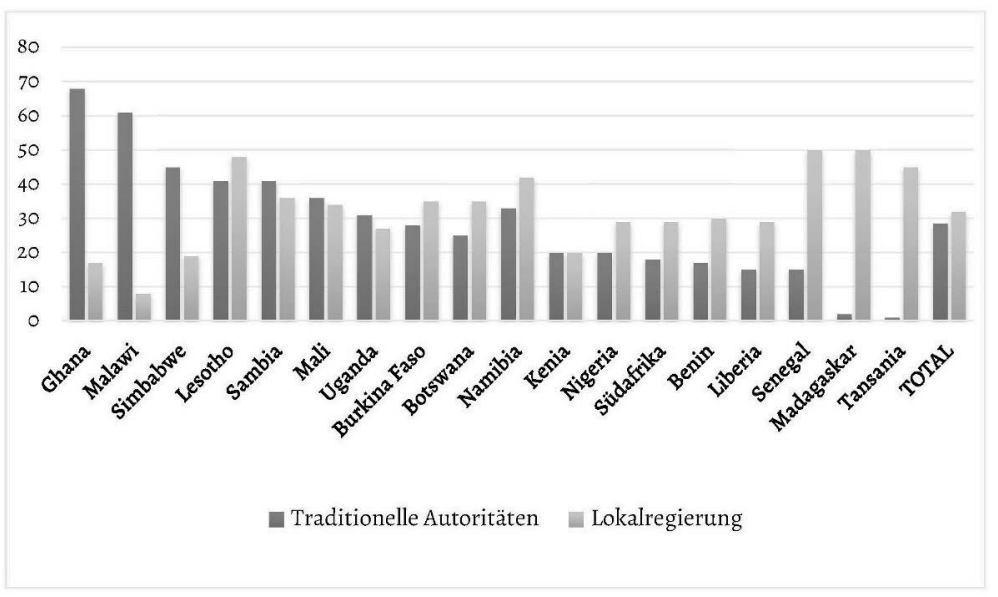

Quelle: Logan 2013: 361, eigene Darstellung, Angaben in Prozent

über mehr als eine Million Hektar Land unterzeichnet (The Land Matrix 2019). Das entspricht dem Vierfachen der Fläche Luxemburgs. Damit liegt Ghana im Vergleich mit anderen Ländern Subsahara-Afrikas vom Flächenausmaß nur hinter Mosambik und Äthiopien.

Wie auch in anderen Ländern treten im Verlauf der Verhandlungen großflächiger Landvergabe und während der Implementierung der Projekte in Ghana häufig Konflikte auf: Konflikte zwischen unterschiedlichen Gruppen von Landnutzer*innen, Konflikte im Zusammenhang mit illegalen Verkäufen von Land oder der Intransparenz der Verhandlungen, und Konflikte zwischen unterschiedlichen Nutzer*innengruppen wie Kleinbäuerinnen und -bauern und mobilen Viehzüchter*innen (Tsikata/Yaro 2011). Traditionelle Autoritäten sind zentrale Akteure in diesen Konflikten. Spätestens seit Beginn der 200oer Jahre gehören sie in Ghana zu den wichtigsten Verhandlungspartner*innen in Landtransaktionen (Cotula et al. 2014). Vor allem im Verhältnis zu staatlichen Institutionen haben traditionelle Autoritäten seither ihre Macht im Bereich der Landvergabe maßgeblich ausgeweitet. Während in vielen anderen Ländern staatliche Institutionen aktiv an Landvergabeprozessen beteiligt sind, 
spielen sie in Ghana nur eine untergeordnete Rolle und agieren zunehmend nur noch als bestätigende Instanzen. Traditionelle Autoritäten hingegen haben aufgrund der gesetzlichen Anerkennung von traditionellem Recht und ihrer uneingeschränkten Zuständigkeit in der Landverwaltung (Republic of Ghana 1992: Art. 267(1)) relativ freie Hand in den Verhandlungen mit Investoren. Zugleich sind sie laut Verfassung verpflichtet, als Treuhänder*innen die Interessen ihrer Gemeinschaft zu vertreten (Republic of Ghana 1992: Art. 36(8)). Ihnen obliegt es, die Zugangsrechte der Landnutzer*innen zu schützen, im Fall des Verlusts von Land für angemessene Entschädigungszahlungen zu sorgen, Kleinbäuerinnen und -bauern in die Verhandlungen miteinzubeziehen und ihnen die Möglichkeit des Widerspruchs oder der Überprüfung von Vereinbarungen zu geben (Elhadary/Obeng-Odoom 2012; Vermeulen/Cotula 2010: 907). In der Praxis kommen sie dieser Aufgabe oftmals jedoch nicht nach, sondern handeln nach eigenem Interesse und ohne die Zustimmung der Landnutzer*innen. Aufgrund ihrer zentralen und ambivalenten Rollen als Verhandlungspartner*innen sowie Treuhänder"innen in der Verhandlung großflächiger Landtransaktionen sind traditionelle Autoritäten in Ghana zunehmend auch Konfliktakteure. Welche Rolle sie in diesen Konflikten spielen, ist in der Forschung bislang kaum untersucht worden (Boamah 2014a: 407). Mit Blick auf die besonders starke Stellung traditioneller Autoritäten und das im Verhältnis hohe zahlen- und flächenmäßig Ausmaß an land grabbing Fällen ist Ghana als Fallstudienland nicht repräsentativ für den gesamten Kontinent, sondern vielmehr ein trouble case, wie Berry (2017: 114f.) es fasst. Jason Seawright und John Gerring sprechen diesbezüglich von einem extremen Fall (extreme case) (Seawright/Gerring 2008:301), einem Fall der sich hinsichtlich zentraler analytischer Dimensionen als besonders stark oder schwach erweist. Als extremes Fallstudienland eignet Ghana sich besonders gut, um eine größtmögliche Menge an Informationen zu einem bisher noch wenig erforschten Phänomen zu erlangen (Flyvbjerg 2011: 16).

\section{Auswahl der Fälle}

Ein Fall ist in meiner Studie ein empirisch beobachtbarer Konflikt um Zugang zu Land, in dem traditionelle Autoritäten als zentrale Konfliktakteure auftreten. Folgende drei Kriterien bestimmten die Auswahl meiner Fälle. Erstens sollten die Fälle sich in Regionen befinden, in denen sich die großflächige agarindustrielle Umnutzung von Land seit Mitte der $2000 e r$ Jahre maßgeblich ausgeweitet hat. Zweitens sollten in diesen Regionen im Zusammenhang 
mit land grabbing vermehrt Konflikte auftreten. Und drittens sollten die Fälle in zwei Regionen liegen, in denen sich die Ausgestaltung der traditionellen Herrschaft unterscheidet. Jenseits dieser Kriterien war der Anspruch an die Fallauswahl, eine größtmögliche Vergleichbarkeit zu gewährleisten. Unter Berücksichtigung dieser Kriterien fiel die Auswahl der Fälle auf die Konflikte um das ScanFarm-Projekt in der Ashanti Region und das BioFuel Africa-Projekt in der Northern Region. Als typical cases (Gerring 2007: 91) sind sie repräsentativ für andere Konflikte, die im Zusammenhang mit land grabbing in Ghana und darüber hinaus entstanden. Dies bezieht sich insbesondere auf den Umsetzungszeitpunkt der Projekte, den Zweck der Umnutzung des Lands, die intendierte Größe der gepachteten Fläche sowie die am Konflikt beteiligten Akteure. In Bezug auf die Ausgestaltung der traditionellen Herrschaft in beiden Forschungsregionen handelt es sich um diverse cases (Gerring 2007: 97f.). Die Mischung aus typical und diverse case leitet sich aus meinem Erkenntnisinteresse sowie meinen theoretischen Vorannahmen ab. Ich möchte Aussagen zum Handeln traditioneller Autoritäten in Konflikten im Kontext von land grabbing treffen, deren Reichweite über die von mir untersuchten Fälle hinausgehen. Aus analytischer Perspektive eignen sich diesbezüglich Fälle, die ein breites Spektrum anderer Fälle repräsentieren, also typische Fälle. Gleichzeitig ist meine Vorannahme, dass Unterschiede in der traditionellen Herrschaft, und damit verbunden das unterschiedliche Handeln traditioneller Autoritäten, Auswirkungen auf die Entstehung und den Verlauf von Konflikten um Zugang zu Land haben. Für die Analyse setzt dies voraus, dass ich mindestens zwei Fälle untersuche, in denen sich die traditionelle Herrschaft unterscheidet. Als Forschungsdesign leitet sich daraus die diverse-case-Strategie $a b$.

$\mathrm{Ab}$ Mitte der 2000er Jahre kam es in vielen Ländern Subsahara-Afrikas und insbesondere in Ghana - zur Ausweitung des großflächigen Anbaus von Energiepflanzen für die Produktion von Agrartreibstoffen. Insbesondere der Anbau von Jatropha erfuhr einen regelrechten Boom. In diesem Kontext kam es vielerorts zu Konflikten um das für den Anbau von Jatropha vorgesehene Land (Campion/Acheampong 2014; Hunsberger 2010; Schrader 2014; von Maltitz et al. 2014). Innerhalb Ghanas variierte die räumliche Ausbreitung des Jatropha-Anbaus erheblich. Im südlichen Ghana konzentrierte sie sich hauptsächlich auf die Regionen Brong-Ahafo und Ashanti und im nördlichen Teil des Landes auf die Regionen Upper West, Northern Region und Upper 
East $^{1}$ (Yirrah 2012). Mittlerweile ist der Jatropha-Boom vorüber. Ehemalige Jatropha-Projekte sind entweder gescheitert oder die für den Jatropha-Anbau eingeplanten Flächen dienen nun dem Anbau anderer Nutzpflanzen. Die Konflikte dauern jedoch in vielen Fällen an. Die Projekte ScanFarm und BioFuel Africa sind repräsentativ für dieses Phänomen und zeichnen sich darüber hinaus durch eine gute Vergleichbarkeit aus. Beide Projekte wurden zwischen 2005 und 2008 von norwegischen Investoren mit dem Ziel des Jatropha-Anbaus initiiert, waren jedoch wie zahlreiche andere Projekte erfolglos mit diesem Vorhaben. ScanFarm produziert seit 2009 Mais und in geringem Umfang unterschiedliche andere Pflanzen für den lokalen Markt und den Export. BioFuel Africa scheiterte 2011 gänzlich. Seither liegen die ursprünglich für den Jatropha-Anbau gepachteten Flächen brach und es ist unklar, wer aktuell über die Nutzungsrechte verfügt. In beiden Konflikten zählen traditionelle Autoritäten zu den zentralen Konfliktakteuren, wobei sich die Ausgestaltung der traditionellen Herrschaft zwischen der Ashanti Region und der Northern Region unterscheidet. In der Ashanti Region sind traditionelle Autoritäten historisch betrachtet und bis in die heutige Zeit besonders einflussreich. Sie sind in stark hierarchischen Strukturen organisiert, die in großem Maß auf die Kolonialzeit zurückgehen. Insbesondere traditionelle Autoritäten in hohen Positionen gehören in der Regel der nationalen Elite an und verfügen über große (internationale) Netzwerke in Politik und Wirtschaft. Im Vergleich dazu unterscheiden sich die drei nördlichen Regionen in der Ausgestaltung der traditionellen Herrschaft. Traditionelle Autoritäten in den nördlichen Regionen sind in der Regel weniger stark in (inter)nationale Netzwerke eingebunden und verfügen im Vergleich über weniger Ressourcen. Die Vergabe von Land liegt jedoch in beiden Regionen gleichermaßen in der Verantwortung traditioneller Autoritäten. Im Dagbon-Königreich, der Forschungsgegend in der Northern Region, haben die paramount chiefs jedoch eine Rechenschaftspflicht gegenüber dem Ya Naa, dem König der Dagbon und der höchsten traditionellen Autoritäten. In der Ashanti Region hingegen können die paramount chiefs Entscheidungen bezüglich der Vergabe von Land ohne Absprache mit dem Asantehene, dem König der Ashanti, beschließen. 


\subsection{Erhebung der Daten}

Die zentralen Methoden der Datenerhebung für meine Studie sind teilstrukturierte Leitfadeninterviews und Gruppendiskussionen. Im Laufe von drei Feldforschungsphasen in den Zeiträumen von November 2015, März bis Juni 2016 und Februar bis März 2017 habe ich insgesamt 46 Interviews mit 52 Personen und sieben Gruppendiskussionen mit insgesamt rund 170 Personen geführt. Hinsichtlich der Auswahl der Interviewpartner*innen verfolgte ich den Anspruch, ein möglichst großes Spektrum an für die Fälle relevanter Akteure abzubilden. Anhand einer umfassenden Literaturauswertung sowie im Verlauf des ersten explorativen Feldforschungsaufenthalts 2015 identifizierte ich sechs zentrale Akteursgruppen: Mitarbeiter*innen staatlicher Institutionen, Mitarbeiter*innen traditioneller (Landrechts-)Institutionen, traditionelle Autoritäten, Landnutzer*innen, Unternehmensakteure sowie zivilgesellschaftliche Akteure. Interviewpartner*innen waren dementsprechend Mitarbeiter*innen staatlicher Institutionen auf nationaler, regionaler und Distriktebene, Repräsentanten der CLS (traditionelle Landrechtsinstitution), Vertreter des National House of Chiefs sowie des Regional House of Chiefs der Northern Region, traditionelle Autoritäten und ihre Berater (elders) sowie Landnutzer*innen der betroffenen Gegenden, leitende Angestellte der Unternehmen und Mitarbeiter*innen zivilgesellschaftlicher Organisationen. Teilnehmende an den Gruppendiskussionen waren die durch die agrarindustriellen Projekte betroffenen Landnutzer*innen und traditionellen Autoritäten der Dörfer Nsonyameye und Dukusen (ScanFarm), Jimle, Kpachaa, Parachanayilli, Tuya, Changolinaaya und Jashei sowie die elders des paramount chiefs von Tijo (BioFuel Africa). Bei der Auswahl der Interviewpartner*innen aus der Gruppe der Landnutzer*innen habe ich auf eine ausgeglichene Verteilung hinsichtlich der Kategorien Herkunft, Alter, Geschlecht und Art des Landzugangs geachtet. In beiden Fällen gab es Landnutzer*innen, die den Zugang zu ihrem Land über eine traditionelle Autorität erhalten hatten und andere, die sich als Nachkommen derjenigen bezeichneten, die das Land als erste bestellt haben. Ihr Zugang zu Land unterliegt somit nicht der Kontrolle durch traditionelle Autoritäten. Darüber hinaus gab es Landnutzer*innen, die ursprünglich aus der Gegend waren und andere die zugezogen waren. In den Einzelinterviews erwies es sich schwierig mit jungen Menschen gleich welchen Geschlechts zu sprechen. In der Regel waren sie aufgrund ihrer Arbeit stark eingebunden und konnten nur wenig Zeit für ein Interview aufbringen. Darüber hinaus zogen sie es meistens vor, den älteren Menschen aus ihrer Familie das Wort 
zu überlassen, da sie selbst ihren Zugang zu Land über Letztere erhielten. In den wenigen Interviews die ich mit jungen Menschen führen konnte, stellte sich letztendlich aber auch heraus, dass sie zu dem Konflikt in Zusammenhang mit der Vergabe des Lands an das Unternehmen nur wenig sagen konnten und mich immer wieder an ältere Menschen aus ihrer Familie verwiesen, die ihrer Meinung nach über ein umfassenderes Wissen verfügten.

Im Folgenden erläutere ich zunächst meine Beweggründe für die Wahl teilstrukturierter Leitfadeninterviews und Gruppendiskussionen als Erhebungsmethoden und lege dar, wie ich bei der Entwicklung der Leitfäden vorgegangen bin. Im Anschluss daran gehe ich näher darauf ein, wie ich mir Zugang zum Feld verschafft habe, wie ich in der Durchführung der Interviews vorgegangen bin und diskutiere die jeweiligen damit verbundenen Herausforderungen. Schließlich erörtere ich meinen Anspruch, mittels Iteration und Triangulation die Qualität der erhobenen Daten abzusichern.

\section{Teilstrukturierte Leitfadeninterviews und Gruppendiskussionen}

Das teilstrukturierte Leitfadeninterview zeichnet sich durch die Qualität »so offen wie möglich, so strukturierend wie nötig« (Helfferich 2014:560) zu sein, aus. Damit ist es als Erhebungsmethode gut geeignet, um bislang wenig erforschten Phänomenen mit großer Offenheit zu begegnen. Für die Entwicklung meiner Leitfäden habe ich mich an Cornelia Helfferichs (2011: 186) Ausführungen orientiert. Die Struktur dieses Leitfadens zeichnet sich dadurch aus, dass sie narrativ ausgelegt ist und den Interviewten dadurch die Möglichkeit gibt, ihre eigene Perspektive darzustellen, eigene Schwerpunkte zu setzen und auf Themenbereiche einzugehen, die der Leitfaden nicht vorgibt. Gleichzeitig ermöglicht die offene und flexible Teilstrukturierung Interviewenden, forschungsrelevante Themenbereiche nicht aus dem Blick zu verlieren und gezielte Nachfragen zu stellen, wenn Interviewte bestimmte relevante Themen selbst nicht ansprechen oder sich das Interview in eine Richtung entwickelt, die forschungsirrelevant ist. In Anlehnung an das von Helfferich entwickelte Schema habe ich die Leitfäden je nach Akteursgruppe in vier bis fünf große Themenkomplexe strukturiert, die ich aus der vorherigen Literaturauswertung und mit Blick auf mein Erkenntnisinteresse entwickelt habe. Jeder Themenkomplex beginnt mit einer Erzählaufforderung. Diese Frage gibt an, in welche Richtung das Forschungsinteresse geht, ist jedoch so offen gestellt, dass sie die Interviewpartner*innen ermuntern soll, ihre eigene Perspektive auf das jeweilige Thema darzustellen. Um forschungsrelevante 
Punkte nicht aus dem Blick zu verlieren, enthält jeder Themenkomplex eine Anzahl an Memos. Dies sind Themen, die idealerweise durch die Interviewten selbst thematisiert werden sollten. Ist dies nicht der Fall, können sie im Verlauf des Interviews an geeigneter Stelle als gezielte Nachfrage integriert werden. Den Abschluss des Interviewleitfadens bildet die Frage nach weiteren relevanten Themen, die im Verlauf des Interviews noch nicht angesprochen wurden. Ziel dieser Frage war es, Raum für Themen zu geben, die im bisherigen Forschungsprozess fehlten, von den Beforschten selbst jedoch als wichtig erachtet werden. Mit Blick auf Themen, welche die Befragten selbst einbrachten und damit zusammenhängenden neuen Erkenntnissen habe ich den Interviewleitfaden im Forschungsprozess immer wieder angepasst und ergänzt.

Den Leitfaden habe ich sowohl für die Einzelinterviews als auch in Gruppendiskussionen verwendet. Gruppendiskussionen habe ich ausschließlich mit den von den agrarindustriellen Projekten betroffenen Landnutzer*innen geführt. In diesem Kontext eigneten sich besonders Gruppendiskussionen als Methode gut, um die kollektive Meinung der Gruppe in Bezug auf den Landnutzungswandel, seine Folgen und Konflikte, die damit in Zusammenhang stehen, herauszuarbeiten. In Gruppendiskussionen steht nicht die gleichzeitige Befragung mehrerer Personen im Vordergrund, sondern die Interaktion der Gruppenmitglieder (Bohnsack 2009: 360; Vogl 2014: 581). Um eine möglichst hohe Informationsdichte $\mathrm{zu}$ erlangen, hätte ich es aus methodischer Sicht vorgezogen, Fokusgruppendiskussionen mit Realgruppen - sowie künstlichen Gruppen von maximal 12 Teilnehmer*innen durchzuführen (Vogl 2014: 584). Realgruppen sind Gruppen, die das reale soziale Gefüge der Gruppe aus der die Interviewpartner*innen stammen, abbilden. In Bezug auf die Landnutzer*innen hätte ich entsprechend innerhalb der Gruppe auf eine Varianz bezüglich Alter und Geschlecht geachtet. Künstliche Gruppen sind Gruppen, die hinsichtlich einer oder mehrerer Kategorien homogen sind. So hätte ich gerne Gruppen aus jungen Frauen, älteren Frauen, Frauen gemischten Alters, jungen Männern, älteren Männern und Männern gemischten Alters gebildet. Das war in der praktischen Durchführung jedoch nicht möglich, da es die Landnutzer"innen in den - meist sehr kleinen Dörfern bevorzugten, gemeinsam interviewt zu werden. 


\section{Feldzugang und Durchführung der Interviews}

Die Grundvoraussetzung für die erfolgreiche Durchführung einer Fallstudie ist der Zugang zum Feld. Diesen erarbeitete ich mir sukzessiv im Verlauf meiner drei Forschungsaufenthalte. Ziel der ersten Forschungsreise war die Sondierung des Forschungsfelds, die Vernetzung mit relevanten Akteuren und die Fallauswahl. Während dieses einmonatigen Aufenthalts im November 2015 traf ich Mitarbeiter*innen zivilgesellschaftlicher Organisationen und Wissenschaftler*innen, die sich mit dem Thema land grabbing sowie damit einhergehenden Konflikten in Ghana auseinandergesetzt hatten. Mein Anliegen war es, ein Kontaktnetzwerk aufzubauen, das hilfreich für die Fallauswahl sowie für den Zugang zum Feld sein würde. Mein erster Zugangspunkt war die NGO Action Aid International Ghana, auf die ich durch die vorherige Literaturauswertung aufmerksam geworden war. Diese stellte den Kontakt zu einem Mitarbeiter des Food Security Policy Advocacy Network (FoodSPAN) her. Gemeinsam hatten beide intensiv zu Konflikten geforscht, die im Rahmen steigender internationaler Investitionen in den Anbau von Energiepflanzen zur Gewinnung von Agrartreibstoffen auftraten und zahlreiche Publikationen dazu veröffentlicht. Mein zweiter Zugangspunkt war die University of Ghana. Dort tauschte ich mich mit drei Wissenschaftler"innen aus, die zahlreiche Artikel zu land grabbing, Konflikten um Land und traditioneller Herrschaft publiziert hatten. Diese bat ich jeweils um weitere Kontaktpersonen, die für mein Forschungsvorhaben relevant sein könnten. So erarbeitete ich mir über das Schneeballverfahren kontinuierlich weitere Kontakte, die mich schließlich maßgeblich in der Auswahl meiner Fälle unterstützten. Als ersten Fall identifizierte ich so das ScanFarm Projekt. Auch während meines zweiten Aufenthalts von Februar bis Juni 2016 hatte ich die Möglichkeit, Kontakte zu Wissenschaftler*innen und Mitarbeiter"innen Zivilgesellschaftlicher Organisationen zu knüpfen. Durch zahlreiche Diskussionen mit unterschiedlichen Gesprächspartner"innen und weitere Literaturrecherche identifizierte ich den zweiten Fall, das BioFuel Africa Projekt.

Ich begann mit der Datenerhebung in Accra, der Hauptstadt Ghanas. Ziel war es, zunächst allgemeine Informationen zu Konflikten im Kontext von land grabbing, traditioneller Herrschaft, Funktionsweise und Zusammenspiel des traditionellen und staatlichen Landrechtssystems sowie zu Entwicklungsund Investitionsstrategien im Landwirtschaftssektor zu erheben. In diesem Rahmen führte ich zwischen 2015 und 2017 zwei Interviews mit Mitarbeiter*innen zivilgesellschaftlicher Organisationen und vier Interviews mit Mit- 
arbeitern staatlicher Stellen. Die zweite Etappe der Feldforschung war Kumasi, die Regionalhauptstadt der Ashanti Region. Dort realisierte ich zunächst ein Interview mit dem Mitarbeiter einer NGO, der über hilfreiche Informationen zum ScanFarm Projekt verfügte und mir diesbezüglich weitere Kontakte vermitteln konnte. Zwischen 2016 und 2017 führte ich in Kumasi des Weiteren drei Interviews mit Mitarbeiter*innen staatlicher Stellen, die in die Vergabe des Lands an ScanFarm involviert waren, sowie mit einem Mitarbeiter des Asantehenes Land Secretariats, der regionalen traditionellen Landrechtsinstitution und einem Mitarbeiter des National House of Chiefs. Die dritte Etappe meiner Feldforschung war Agogo, die Hauptstadt des Asante Akyem North Districts, wo sich das ScanFarm Projekt befindet. Dort führte ich 2016 und 2017 zwei Interviews mit dem CEO von ScanFarm und zwei mit Mitarbeitern traditioneller (Landrechts-)Institutionen. Außerdem war es mir möglich zwei Interviews mit staatlichen Akteuren auf Bezirksebene zu realisieren. Die Sprache dieser Interviews war ausschließlich Englisch mit Ausnahmen desjenigen mit den Mitarbeitern traditioneller (Landrechts-)Institutionen, die es vorzogen auf Twi zu kommunizieren. Bei der Übersetzung hatte ich Hilfe durch einen Forschungsassistenten.

Den Großteil meiner Interviews in Agogo und den durch das ScanFarm-Projekt betroffenen Dörfern führte ich mit den traditionellen Autoritäten der jeweiligen Dörfer und den Landnutzer*inenn. Den Zugang zu diesen Interviewpartner*innen erschloss ich mir über unterschiedliche Wege. Den Erstkontakt zu den chiefs von Dukusen, Nsonyameye und Baamaa sowie $\mathrm{zu}$ einigen Landnutzer*innen stellte ein Mitarbeiter des Department of Agriculture her, der für dessen Community-Arbeit zuständig war und einen guten Zugang zu den Landnutzer*innen hatte. Er leistete auch die organisatorische Vorarbeit für eine Gruppendiskussion. Weitere Kontakte zu Landnutzer*innen erhielt ich über das Schneeballverfahren. So entstand auch der Kontakt zu den Agogoman mma kuo (weltweite Bürger*innen von Agogo). Die Agogoman mma kuo ist ein zivilgesellschaftliches Netzwerk engagierter Bewohner*innen von Agogo, das bereits vor der Implementierung des ScanFarm Projekts existierte und sich mittlerweile für die Interessen einiger Landnutzer*innen einsetzt. Insgesamt realisierte ich 14 Interviews mit Landnutzer*innen. Bis auf ein Interview, das in englischer Sprache stattfand, führte ich diese Interviews auf Twi mit einer Übersetzung durch meinen Forschungsassistenten.

Für die Datenerhebung zum Konflikt um BioFuel Africa konnte ich den Leiter der NGO Northern Accelerated Intervention for Development als Forschungsas- 
sistenten gewinnen, der mit dem Umfeld und dem lokalen Verhaltenskodex vertraut war. Die Zusammenarbeit mit ihm vereinfachte den Zugang zum Feld. Er vermittelte mir Kontakte zu einigen staatlichen Stellen, zum CLS in der Regionalhauptstadt Tamale sowie zu einem leitenden Angestellten des Unternehmens. Darüber hinaus leistete er die organisatorische Vorarbeit für ein Interview und insgesamt sechs Gruppendiskussionen mit traditionellen Autoritäten und Landnutzer*innen in sieben Dörfern der Gegend des BioFuel Africa Projekts. Mit einigen anderen staatlichen Stellen und dem Regional House of Chiefs vereinbarte ich eigenständig Interviewtermine. Insgesamt realisierte ich so während der Feldforschungsaufenthalte 2016 und 2017 fünf Interviews mit Mitarbeiter*innen regionaler staatlicher Institutionen, zwei Interviews mit Repräsentanten formalisierter traditioneller Institutionen auf regionaler Ebene und ein Interview mit einem Angestellten des Unternehmens. Diese Interviews führte ich allein und auf Englisch durch. Das Interview mit zwei Landnutzern aus der betroffenen Gegend sowie die sechs Gruppendiskussionen mit Landnutzer*innen und traditionellen Autoritäten fanden auf Dagbani mit Übersetzung ins Englische durch meinen Forschungsassistenten statt.

In allen bis auf zwei Fällen willigten die Interviewpartner*innen in die Audioaufzeichnung des Interviews ein. Zu den anderen Interviews sowie den Gruppendiskussionen, die nicht für eine Audioaufzeichnung geeignet sind, fertigte ich Gesprächsprotokolle an.

\section{Qualitätssicherung der Daten durch Iteration und Triangulation}

Die Aussagen von Interviewpartner*innen spiegeln immer ihre persönliche Wahrnehmung und Einschätzung wider und können daher als »subjektive Wahrheit« verstanden werden, »die für den spezifischen Erzählaugenblick gültig ist« (Helfferich 2014: 561). Um dennoch Vertrauen in die eigenen Daten $\mathrm{zu}$ entwickeln, habe ich in meiner Forschung Iteration und DatenTriangulation angewandt. Iteration bedeutet, vielen unterschiedlichen Interviewpartner"innen die gleichen Fragen zu stellen, bis ein gewisser Sättigungsgrad an Informationen erreicht ist (Lund 2014: 226f.). Triangulation bedeutet die empirischen Daten mit anderen Quellen abzugleichen, um so mindestens zwei Sichtweisen auf den gleichen Untersuchungsgegenstand $\mathrm{zu}$ erhalten (Yin 2009). Während Iteration zur Bestätigung von Aussagen nützlich sein kann, eignet sich Triangulation zur Generierung weiterer Erkenntnisse (Flick 2014: 419). Daten-Triangulation »kombiniert Daten, die 
verschiedenen Quellen entstammen und zu verschiedenen Zeitpunkten, an unterschiedlichen Orten oder bei verschiedenen Personen erhoben werden« (Flick 2014: 418). So habe ich im Vorfeld und im Verlauf der empirischen Datenerhebung eine umfassende Analyse wissenschaftlicher Literatur zu meinem Forschungsfeld durchgeführt und habe Gesetzestexte, Zeitungsartikel, Blog-Beiträge, Berichte zivilgesellschaftlicher Organisationen, graue Literatur und Unternehmensberichte ausgewertet. Die Berücksichtigung und Auswertung zusätzlicher Quellen in Ergänzung zur eigenen Forschung stärkt die Plausibilität der eigenen empirischen Daten, dient aber nicht ihrer abschließenden Validierung (Lund 2014: 227). Sie können jedoch hilfreich sein, um die Bedeutung von Aussagen zu überprüfen, unterschiedliche Perspektiven auf das gleiche Phänomen aufzudecken, und die Reproduzierbarkeit einer Beobachtung oder Interpretation $\mathrm{zu}$ belegen (Stake 2003: 148).

\subsection{Reflexion des methodischen Vorgehens}

Im Verlauf der Forschung habe ich einige Herausforderungen hinsichtlich des methodischen Vorgehens identifiziert. Diese reflektiere ich im Folgenden und gehe auf meinen Umgang mit ihnen ein. Zentrale Herausforderungen waren mit der Übersetzung der Interviews verbunden, mit der fehlenden Bereitschaft der Partizipierenden, Einzelinterviews zu geben und mit ungleichen Redeanteilen der einzelnen Akteure in Gruppendiskussionen. Nicht zuletzt erwies sich auch der Vergleich der beiden Fälle als herausfordernd.

Während Mitarbeiter*innen staatlicher Stellen und zivilgesellschaftlicher Organisationen in der Regel sehr gut Englisch sprechen, bevorzugte ein Großteil der Landnutzer*innen und traditionellen Autoritäten die Kommunikation in ihrer jeweiligen Erstsprache. In der Ashanti Region war dies Twi, in der Northern Region Dagbani. Für Interviews und Gruppendiskussionen, die ich mit diesen Akteuren führte, war daher immer eine Übersetzung nötig. Diese beeinflusst immer auch die Gesprächsatmosphäre und birgt das Risiko, sich hemmend auf den Gesprächsfluss auszuwirken. Die meisten Interviewten sind jedoch mit diesem Umstand vertraut und äußerten sich sehr offen. Sonstige Schwierigkeiten, die mit der Übersetzung verbunden sind, habe ich im Vorfeld detailliert mit meinen Forschungsassistenten besprochen. Besonderen Wert habe ich darauf gelegt, dass meine Fragen und die Antworten der Interviewten immer im O-Ton übersetzt wurden und dass die Antworten 
nicht in zusammenfassender Form wiedergegeben werden, damit auch die Argumentationsstruktur nachvollziehbar bleibt.

Die Interviews und Gruppendiskussion, die ich mit den Landnutzer*innen in der Ashanti Region führte, zeichneten sich durch eine große Offenheit von Seiten der Interviewpartner*innen aus, die mir bereitwillig Auskunft zu meinen Fragen gaben. In der Northern Region erwies sich die Forschungssituation als schwieriger. Im Allgemeinen standen mir die meisten Landnutzer*innen zunächst sehr viel skeptischer und verhaltener gegenüber. Um eine Vertrauensbasis zu schaffen, berichtete ich ausführlich über meine Forschung und gerantierte ihnen ihre Anonymität. Das entspannte die Atmosphäre maßgeblich. Letztendlich entstand so eine größere Offenheit seitens der Landnutzer*innen und es stellte sich heraus, dass einige von ihnen bereits schlechte Erfahrungen mit Forscher*innen gemacht hatten. Vor allem waren sie aber nicht bereit Einzelinterviews zu geben. Da sie in sehr kleinen Dörfern zusammenleben, zogen sie es vor, dass alle Dorfbewohner*innen im Rahmen von Gruppendiskussionen am Forschungsprozess teilhaben konnten. Auf diese Weise wollten sie gewährleisten, dass sie alle dieselben Informationen erhielten und gleichermaßen die Möglichkeit bekamen, ihre Meinung zu äußern. Dennoch variierten in einigen von mir geführten Gruppendiskussionen die Redeanteile der teilnehmenden Personen mitunter beträchtlich, worauf ich mich jedoch bereits im Vorfeld eingestellt hatte. Denn Gruppendiskussionen sind immer durch Machtverhältnisse unter den Teilnehmenden strukturiert, die bestimmen, wer sich in welchem Umfang zu Wort meldet, wer in der Lage ist, die eigene Meinung anderen gegenüber offenzulegen und zu vertreten. Problematisch an Gruppendiskussionen ist also, dass die öffentliche Diskussion die Äußerung individueller, eventuell von der Mehrheit abweichender Meinungen, verhindern kann (Vogl 2014: 582). Insbesondere Frauen und junge Menschen meldeten sich in diesen Fällen weniger zu Wort. Traditionelle Autoritäten und ältere Männer dominierten die Diskussion in der Regel. Wenn die Gesprächsatmosphäre und Haltung der anderen Teilnehmenden es zuließen, habe ich gegen Ende der Gruppendiskussion explizite Erzählaufforderungen an Gruppen mit geringem Redeanteil gerichtet. Um Situationen wie diese in der Zukunft zu umgehen, könnte eine Möglichkeit sein, aus der großen Gruppe Kleingruppen zu bilden und diese in weibliche, männliche und gemischte Teilnehmer*innen zu unterteilen. Vor allem in Frauengruppen könnte es darüber hinaus sinnvoll sein, eine Übersetzerin zu engagieren, da die Anwesenheit von Männern in einigen Kontexten zu einer verhaltenen Gesprächsatmosphäre bei Frauen führt. 
$\mathrm{Zu}$ guter Letzt erwies sich der Vergleich der beiden Fälle als herausfordernd. Die Stärke eines Paarvergleichs liegt in der ausgeglichenen Kombination aus deskriptiver Tiefe und analytischer Herausforderung (Tarrow 2010: 246), die weder Einzelfall- noch large-N-Studien bieten können. Der Vergleich kann sich jedoch im Laufe der Forschung teilweise als schwierig erweisen. In den von mir untersuchten Fällen ergaben sich die Herausforderungen des Vergleichs insbesondere aus den regionalen Unterschieden, deren eventuelle Auswirkungen auf die Konflikte und auf meine Forschung ich zu Beginn der Konzeption meiner Untersuchung nicht berücksichtigt habe. Der Norden Ghanas ist sehr viel strukturschwächer als der Süden. Staatliche und formalisierte traditionelle Institutionen, wie die District Assembly oder das CLS, sind in der Northern Region weniger vorhanden als in der Ashanti Region. Die betroffenen Dörfer im BioFuel Africa Fall sind sehr viel verstreuter als im Fall des ScanFarm-Projekts, das formale Bildungssystem ist schwächer und ökonomische Alternativen zur Landwirtschaft sind geringer. Nicht zuletzt aufgrund dieser Faktoren konzentriert sich die wissenschaftliche Forschung in Ghana in den letzten Jahren auf den benachteiligten Norden, was sich in der Haltung der Menschen gegenüber Forscher*innen widerspiegelt: Sie sind "forschungsmüde", wie es eine Kollegin der University of Ghana in Accra ausdrückt. Seit einiger Zeit ist ihre Motivation, bereitwillig Auskunft gegenüber Forscher*innen zu geben, stark gesunken, da sie das Gefühl haben viel zu geben, jedoch nichts zurückzubekommen. Daher war es notwendig, zu Beginn der Befragung der Landnutzer*innen im Dialog mit ihnen herauszufinden, was ihre Erwartungen an meine Forschung sind und transparent zu machen, inwiefern ich diese erfüllen kann oder auch nicht. Des Weiteren könnte der Vergleich der Fälle als schwierig angesehen werden, da eines der beiden Projekte - das BioFuel Africa-Projekt - zum Zeitpunkt der Forschung bereits seit mehreren Jahren beendet war. Dies war jedoch unproblematisch, da ich mich in meiner Untersuchung auf den Zeitraum der Verhandlung der Landvergabe, der Aneignung der Kontrolle über das Land durch den Investor und der Projektimplementierung konzentriere. Unter Berücksichtigung dieser Faktoren, die ein Teil der Forschungsrealität sind, sehe ich die beiden Fälle weiterhin als sehr gut vergleichbar an. 


\subsection{Auswertung und Analyse der Daten}

Mit dem Einverständnis der Interviewtpartner*innen habe ich 40 der insgesamt 47 Interviews aufgezeichnet und anschließend transkribiert. Durch die Aufzeichnung der Interviews konnte ich meinen Gesprächspartner*innen ungeteilte Aufmerksamkeit schenken und gleichzeitig die Fragebögen im Blick behalten. Sieben Interviewpartner*innen entschieden sich gegen eine Aufnahme. $\mathrm{Zu}$ diesen Interviews sowie zu den sieben Gruppendiskussionen habe ich Gesprächsprotokolle angefertigt. Da die meisten dieser Interviews eine Übersetzung aus dem Twi oder Dagbani ins Englische benötigten, hatte ich ausreichend Zeit zur Verfassung der Notizen. Um den Gesprächskontext später gut rekonstruieren zu können, habe ich im Anschluss an jedes Interview zudem eine kurze Beschreibung der Gesprächssituation und -atmosphäre verfasst.

Meine empirischen Daten habe ich mit MaxQDA, einer Software für die computergestützte Analyse qualitativer Daten, ausgewertet. Methodisch orientierte ich mich bei Auswertung der Daten an der qualitativen Inhaltsanalyse (Mayring 2014; Mayring/Fenzl 2014). Die qualitative Inhaltsanalyse ist eine qualitativ-interpretative Methode der Auswertung von Textmaterial, die sich auch zur Erfassung latenter Sinngehalte eignet. Gegenüber anderen Ansätzen der Textanalyse unterscheidet sich die qualitative Inhaltsanalyse dadurch, dass sie entlang klar definierter Kategorien erfolgt. Kategorien können als Kurzformulierungen von Analyseaspekten verstanden werden, deren hierarchische Anordnung in Ober- und Unterkategorien möglich ist (Mayring/Fenzl 2014: 544). Die Kategorien können während der Auswertung induktiv am Material entwickelt sowie vorab deduktiv aus der Theorie hergeleitet werden. Die Zusammenstellung aller für die Analyse relevanten Kategorien im Kategoriensystem, ist der grundlegende Schritt für die Auswertung der Daten. Das Kategoriensystem bildet das zentrale Instrumentarium für die Analyse. Diese berücksichtigt nur Textstellen, denen Kategorien zugeordnet werden können (ebd.). Die Zuordnung der Kategorie zu den entsprechenden Textstellen erfolgt nach zuvor festgelegten Zuordnungsregeln. Der Vorteil dieser Methode der Datenauswertung ist, dass sie streng regelgeleitet verläuft und damit intersubjektiv überprüfbar ist (ebd.: 543).

Für die Entwicklung meines Kategoriensystems habe ich mich am Ablaufmodell induktiver Kategorienbildung und deduktiver Kategorienanwendung von Philipp Mayring und Eva Brunner (2006) orientiert (Abb. 3). Nach diesem Modell folgt als erster Schritt nach der Bestimmung des Ziels der Analyse die 
Festlegung der Analyseeinheiten, die später den Kategorien zugeordnet werden. Als kleinste Einheit legte ich einen Satz fest. Für die Analyse muss dieser immer im Kontext des Absatzes, in den er eingebettet ist, interpretiert werden. Im zweiten Schritt erarbeitete ich mein Kategoriensystem. Dafür leitete ich zunächst deduktiv Kategorien aus dem meiner Studie zugrundeliegenden theoretischen Rahmen ab. Diese Kategorien definierte ich, ordnete ihnen jeweils ein Ankerbeispiel zu und legte eine Kodierregel fest, um sie von anderen Kategorien abzugrenzen. Entlang der deduktiv entwickelten Kategorien begann ich mit der strukturierenden Inhaltsanalyse (Mayring 2002). In dessen Verlauf erweiterte ich die bestehenden Kategorien induktiv um neue Unterkategorien. Die induktiven Kategorien bildete ich aus dem Material heraus, indem ich Themen, Aussagen oder Einstellungen, die wiederholt auftauchten, in Kategorien zusammenfasste. Auch diese neu aus dem Material entwickelten Kategorien definierte ich und ordnete ihnen ein Ankerbeispiel und eine Kodierregel zu. Nachdem ich etwa 30 Prozent des Materials kodiert hatte, überarbeitete ich mein Kategoriensystem noch einmal. Eine weitere Überarbeitung nahm ich nach etwa der Hälfte des Materials vor. Die Validität der Datenkodierung sicherte ich mittels Intrakoderübereinstimmung. Nach der vollständigen Kodierung des Datenmaterials kodierte ich dafür erneut sechs Interviews, die ich nach dem Zufallsprinzip aus jeder Akteursgruppe auswählte, ohne die zuvor kodierten Dokumente eingesehen zu haben. Ich verglich beide Dokumente und glich in der abschließenden Überarbeitung des gesamten Materials bestimmte Zuordnungen, die sich unterschieden oder doppelten, noch einmal an. 


\section{Abbildung 3: Ablaufmodell induktiver Kategorienbildung und deduktiver Kategorien- anwendung}

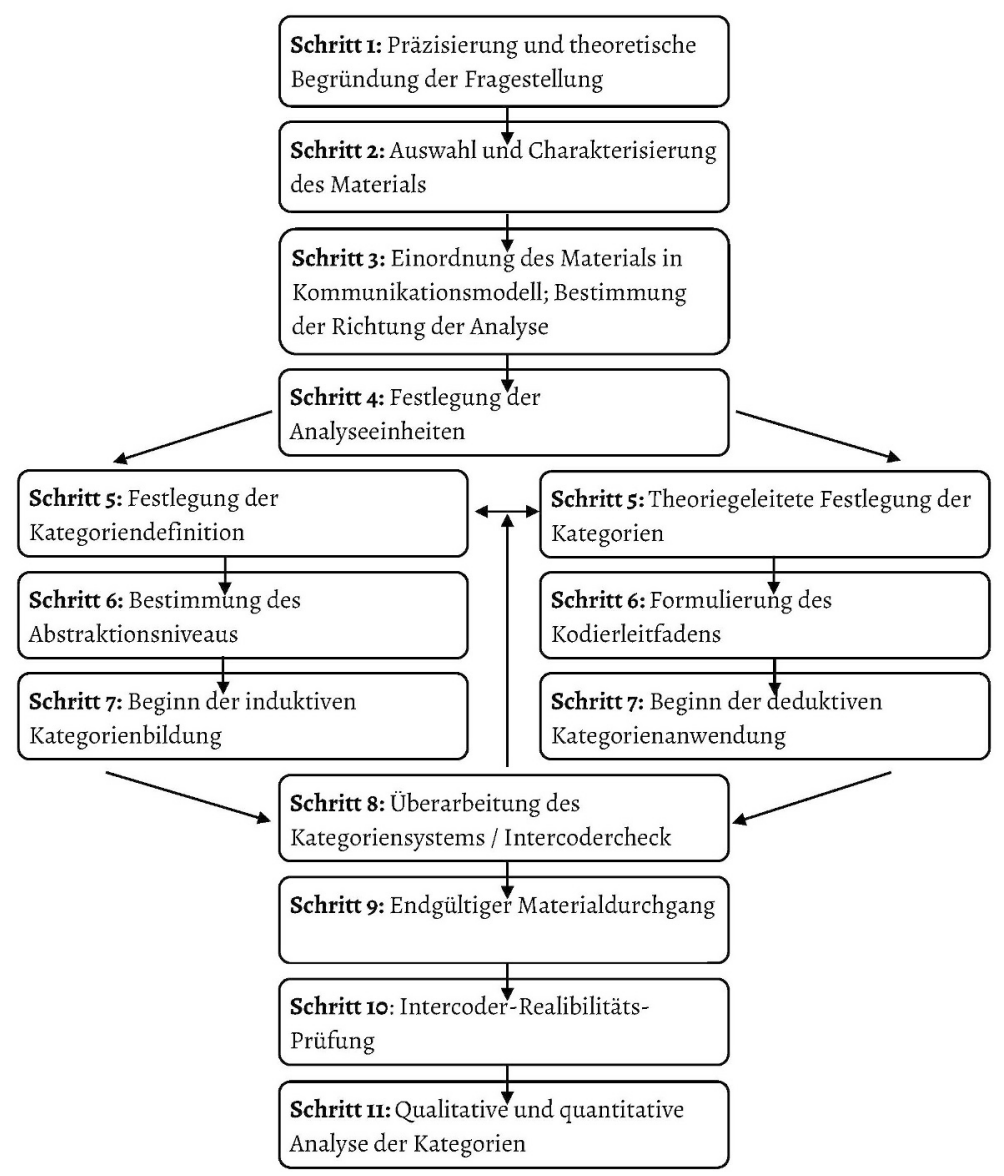

Quelle: Mayring/Brunner 2006 
Folgende Ober- und Unterkategorien leitete ich deduktiv aus der theoretischen Grundlage ab:

\section{Rechtebasierte Zugangsmechanismen}

- Traditionelle Rechte

- Staatliche Rechte

\section{Relationale und strukturelle Zugangsmechanismen}

- Beziehungen

- Netzwerke

- Soziale Zugehörigkeit

\section{Veränderung des Zugangs zu Land im Kontext der Landvergabe}

- Veränderung des rechtebasierten Zugangs

- Veränderung des relationalen und strukturellen Zugangs

\section{Ausübung von Macht und Kontrolle des Zugangs zu Land}

- Sichtbare Macht

- Versteckte Macht

- Unsichtbare Macht

Im Verlauf der induktiven Kategorienentwicklung identifizierte ich keine neuen Oberkategorien, sondern erweiterte die bereits bestehenden deduktiven Kategorien induktiv durch Unterkategorien. Ich differenzierte die rechtebasierten Zugangsmechanismen aus und ergänzte traditionelle Landrechte um die Unterkategorien Gewohnheitsrechte, individuelle Abkommen und formalisierte Landrechte. Staatliche Rechte unterteilte ich in staatlich registrierte traditionelle Landrechte und staatlich registrierte Pachtverträge. Des Weiteren identifizierte ich induktiv Unterkategorien für soziale Zugehörigkeit. Dies waren Geschlecht, Alter, Herkunft und Bildung. Persönliche Beziehungen untergliederte ich in Beziehungen $\mathrm{zu}$ traditionellen Autoritäten, Familienangehörigen und Bekannten. Als Unterkategorien für Netzwerke ergaben sich über die Datenauswertung die Kategorien traditionelle, professionelle und zivilgesellschaftliche Netzwerke. 
Auch für die drei Machtformen entwickelte ich induktiv Unterkategorien. Für die sichtbare Macht waren das Anordnungen gegen den Willen anderer und die Bekräftigung von Entscheidungen gegen Widerstreben. Für die versteckte Macht habe ich die Unterkategorien Ausschluss von Gegner*innen aus Entscheidungsräumen, Bestimmung institutioneller Verfahrensweisen, Beschränkung der Entscheidungsmöglichkeiten von Gegner*innen sowie Kontrolle von Informationen identifiziert. Die unsichtbare Macht habe ich in die Unterkategorien Akzeptanz widersprüchlichen Handels aufgrund gesellschaftlicher Normen, Betonung von Vorteilen trotz verletzter ethischer Interessen und Widersprüche zwischen Handeln und kontrafaktischem Handeln untergliedert.

Die Datenauswertung mit MaxQDA ermöglichte es mir, Zusammenhänge zwischen einzelnen Interviews sowie zwischen Analysekategorien zu erkennen. Die Zusammenhänge zwischen den Interviews waren relevant für den Vergleich der beiden Fälle und erlaubten es, die Unterschiede und Gemeinsamkeiten bezüglich des Handelns traditioneller Autoritäten in Konflikten um Zugang zu Land herauszuarbeiten. Querverbindungen zwischen Analysekategorien lieferten Erkenntnisse bezüglich des Verhältnisses, in dem die unterschiedlichen Zugangsmechanismen zu einander stehen und der Implikationen, die sich daraus für die Kontrolle des Zugangs zu Land ergeben. Ebenso halfen sie dabei, ein Verständnis dafür zu entwickeln, wie sich die unterschiedlichen Machtformen gegenseitig bedingen. Über das Verständnis all dieser Zusammenhänge konnte ich Hypothesen zur Bedeutung des Handelns traditioneller Autoritäten für die Entstehung und den Verlauf von Konflikten um Zugang zu Land bilden.

Die Ergebnisse meiner empirischen Analyse stelle ich in den Kapiteln 7 und 8 dar. Im folgenden Kapitel gebe ich zunächst einen Überblick über den Länderkontext Ghanas mit Schwerpunkt auf Landrechten und der Entwicklung des chieftaincy-Systems. Anschließend daran stelle ich in Kapitel 6 die beiden empirischen Fälle - die Konflikte um ScanFarm und BioFuel Africa vor. 



\section{Länderkontext - Traditionelle Landrechte und das chieftaincy-System in Ghana}

Ziel dieses Kapitels ist es, einen Überblick über die historische Entwicklung der Landrechte und die Bedeutung traditioneller Autoritäten in Ghana zu geben. Besonderes Augenmerk liegt auf der Darstellung traditioneller Landverwaltungssysteme, dessen Verständnis grundlegend für die spätere Analyse ist.

\subsection{Landrechte und die koloniale Erfindung von Traditionen}

Seit Beginn der Kolonialisierung strebte die Britische Krone danach, Zugang und Nutzung von Land zu kontrollieren, um über die Vergabe von Bergbaukonzessionen und Landverkäufen an der florierenden Goldförderung und den Einnahmen aus der Kakaoproduktion teilhaben zu können (Amanor 2005). Geschäfte mit landwirtschaftlichen Nutzflächen nahmen in der heutigen Volta Region im Südosten Ghanas bereits in den frühen Jahren des 19. Jahrhunderts zu, als Palmöl zu einem gefragten Rohmaterial für die industrielle Weiterverarbeitung wurde. Diese Entwicklung verstärkte sich in den 1890er Jahren, in denen Kakao Palmöl als wichtigste Exportpflanze ersetzte. Die 1890er Jahre waren zudem eine Zeit intensiver Goldspekulation, in der sich mehr als 400 Bergbauunternehmen an der Goldküste niederließen und große Waldflächen aneigneten (Amanor 1999: 46). Die fortschreitende Kommodifizierung von Land missfiel der Kolonialverwaltung, da sie selbst nicht daran beteiligt war und kaum eine Möglichkeit hatte, die Ausweitung der kapitalistischen Produktion zu regulieren und Regierungseinnahmen daraus zu generieren. 1884 versuchte die Kolonialregierung daher den Crown Lands Bill einzubringen. Dieses Gesetz sollte »ungenutztes« Land (so genanntes waste land) - unbewirtschaftetes Land, auf das niemand einen formalisierten Rechtsanspruch 
hatte -, sowie Forstflächen und Mineralien unter die Kontrolle der Britischen Krone bringen. Diese sollte fortan das Recht haben, auf unbewirtschaftetem Land Bergbaukonzessionen an internationale Unternehmen zu erteilen. Traditionelle Landnutzer*innen sollten das Land weiterhin bestellen dürfen, ohne jedoch die Möglichkeit zu haben, Landrechte an Fremde - insbesondere europäische Investoren - zu vergeben (ebd.). Der Erlass des Crown Lands Bill scheiterte jedoch am Widerstand eines Zusammenschlusses aufstrebender Intellektueller. Sie argumentierten, jedes Stück Land habe eine*n Besitzer"in. In Folge des anhaltenden Widerstands annullierte die Kolonialregierung den Crown Lands Bill und ersetzte ihn 1897 durch den Lands Bill. Dieser unterschied sich im Wesentlichen dadurch, dass die Kolonialregierung den umstrittenen Begriff waste land ausließ und stattdessen Anspruch auf die Kontrolle über öffentliches Land (public land) erhob. Der Lands Bill gestand den chiefs weiterhin Rechte an Land $\mathrm{zu}$, die ihnen ermöglichten Land an andere Afrikaner*innen, nicht jedoch an Europäer*innen zu vergeben. Afrikaner*innen konnten generell nur temporäre Rechte (settler rights) erlangen. Vererbbare Eigentumsrechte, die auf britischem Recht beruhten, konnte allein die Kolonialregierung vergeben. Auch gegen den Lands Bill gab es erheblichen Widerstand, insbesondere seitens der chiefs und der aufstrebenden intellektuellen Handelsklasse der Goldküste. Sie gründeten gemeinsam die Aborigines' Rights Protection Society, deren Ziel es war, die Interessen der chiefs sowie der kolonialisierten Bevölkerung der Goldküste zu vertreten (Amanor 2005: 103). Die Alternative zum gescheiterten Lands Bill stellte für die Kolonialregierung ein System der Native Administration dar, das sie 1927 mit der Native Administration Ordinance verkündeten. Dieses System baute auf den Prinzipien der für die britische Kolonialmacht typischen indirekten Herrschaft (indirect rule) auf (Crowder 1964). Im Rahmen dieser übte die Kolonialregierung Macht über bereits bestehende örtliche und traditionelle Herrschaftsstrukturen aus. Beruhend auf dem System der indirekten Herrschaft schuf die britische Kolonialregierung in Ghana ein Landnutzungssystem, das Gewohnheitsrecht aufrechterhielt, gleichzeitig aber neue formal-staatliche Gesetze nach englischem Recht einführte (Aryeetey 2007: 11). Das ermöglichte der Kolonialregierung, traditionelle Autoritäten - die bisher in den Widerstand eingebunden waren - als wichtige Verbündete $\mathrm{zu}$ gewinnen und in ihr Herrschaftssystem $\mathrm{zu}$ integrieren. Für die Aufgabe der Landverwaltung in den südlichen Regionen wählte die Kolonialregierung unter den bestehenden traditionellen Autoritäten einer Gegend eine Person aus, die sie zum paramount chief ernannte. In den nördlichen Regionen unterschied sich das Vorgehen, da dort bislang kein chieftaincy-System 
wie im Süden bestand, sondern spirituelle Führer (earth priests) das Land treuhänderisch für lokale Gemeinschaften verwalteten (Lund 2008). Diese ignorierte die Kolonialregierung in ihrem Bestreben, Kontrolle über Land zu erlangen und ernannte stattdessen einflussreiche Kaufleute sowie andere reiche und mächtige Personen zu paramount chiefs (Kasanga/Kotey 2001: 13f.; ObengOdoom 2016: 666). Paramount chiefs bestimmten fortan - kontrolliert durch die Kolonialmacht - die Ausgestaltung von Landverhältnissen, indem sie Verordnungen erließen und festlegten, welche Regeln und Normen in Bezug auf die Landnutzung als gewohnheitsrechtlich anzusehen waren (Boone 2015: 174). Dabei handelte es sich im Wesentlichen um die »Erfindung von Traditionen« (Ranger 1983), auf die sich Kolonialregierung und traditionelle Autoritäten verständigten (Amanor 2008: 63f.). Dies war problematisch, da der chief nicht zwangsläufig die gleiche Vorstellung traditioneller Rechte wie andere Mitglieder der Gemeinschaft hatte (Obeng-Odoom 2016: 665). In einigen Fällen machten chiefs gegenüber der Kolonialregierung auch vorsätzlich falsche Angaben zum Verlauf von Grenzen, um ihre territoriale Macht auszuweiten (Lentz 2010: 74). Kooperationsunwillige oder widerständige chiefs enthob die Kolonialmacht (teilweise gewaltsam) ihres Amts. Anderen, die sich ihr verbunden zeigten, verhalf sie durch Unterstützung in der Beseitigung von Rivalen zur Ausweitung ihrer (territorialen) Macht (von Trotha 1996: 80). Die indirekte Herrschaft über traditionelle Autoritäten ermöglichte es der Britischen Krone, Kontrolle über Land zu erlangen (Ubink 2008a: 22). Gleichzeitig führte dieses System zu einer gestärkten Position traditioneller Autoritäten in der Landvergabe, die über die Kolonialzeit hinaus Bestand hatte. Narrative, die das ideologische Konstrukt aufrechterhalten, dass traditionelle Autoritäten - und nicht die Landnutzer*innen - die Eigentümer*innen des Lands seien, legitimieren bis heute die Landvergabe durch traditionelle Autoritäten. Diese Narrative beschreiben jedoch keine prä-koloniale Realität, sondern reproduzieren koloniale Praxis (Berry 2013: 37).

\subsection{Traditionelle Herrschaft und Landrechte im postkolonialen Ghana}

Mit dem Ende der Kolonialzeit und in den ersten Jahren der Unabhängigkeit änderte sich das ghanaische Landrecht zunächst nicht. Obwohl die unterschiedlichen Regierungsparteien sich in ihrer Ausrichtung deutlich unterschieden, strebten sie keine Veränderung der Landpolitik an. Einzig der So- 
zialist Kwame Nkrumah, Ghanas erster Präsident nach der Unabhängigkeit, bildet hier eine Ausnahme. Er forderte eine Befreiung von traditionellem Elitismus (Nkrumah 1961) und versuchte die wirtschaftliche Macht der chiefs zu brechen, indem er ihnen das formelle Recht absprach, Land zu verwalten und Ansprüche auf Pacht oder anderen Einnahmen zu erheben (Ubink 2008c: 10). Mit dem Administration of Lands Act (Act 123) von 1962 übertrug er die Landverwaltung staatlichen Institutionen. Strukturell änderte sich dadurch jedoch nichts und das System der Landverwaltung blieb in seiner vorherigen Form mehr oder weniger bestehen. Denn obwohl der Staat die Verwaltung von Land übernahm, kamen die Einnahmen daraus letztendlich weiterhin den chiefs zugute (Obeng-Odoom 2016: 667). Nkrumahs kritische Haltung gegenüber traditioneller Herrschaft zog somit de facto keine Einschränkung der Macht der chiefs in der Landverwaltung nach sich. Bis auf kleinere Gesetzesänderungen veränderte sich das Landrecht nach dem Ende der Kolonialzeit bis in die 1980er Jahre kaum (ebd.).

\section{Landreformen und die erneute Stärkung traditioneller Herrschaft}

Erst seit den späten 1980er Jahren unter Präsident Jerry John Rawlings erlebte das Landverwaltungssystem größere Veränderungen. Diese waren Teil einer Welle von Landreformen, die insbesondere internationale Geber- und Finanzinstitutionen wie die Weltbank auf dem afrikanischen Kontinent förderten. Befürworter*innen sahen diese Reformen als wichtigen Schritt für die Sicherung von Landrechten und dadurch einen besseren Zugang zu Krediten, höhere Bildungschancen und die Verringerung von Armut (World Bank 2004). Kritiker"innen hingegen argumentierten, die Reformen kämen hauptsächlich internationalen Unternehmen zugute, die Land auf Kosten der lokalen Bevölkerung aneigneten, um landwirtschaftliche Nutzflächen in Investitionsflächen umzuwandeln (Elhadary/Obeng-Odoom 2012: 59).

Zeitgleich mit der Ausarbeitung von Landreformen gewannen traditionelle Autoritäten - denen seit dem Ende der Kolonialzeit wenig Aufmerksamkeit zuteilwurde - wieder an Einfluss (Buur/Kyed 2007; Englebert 2002b). Trotz staatlicher Bestrebungen, traditionelle Herrschaft zu schwächen, wies diese über die Jahre eine beachtliche Resilienz auf (Ubink 2008c). Traditionellen Autoritäten gelang es sukzessive mehr Einfluss über informelle bottom-up-Prozesse zu erlangen, indem sie sich in Organisationen engagierten oder in größeren ökonomischen Netzwerken zusammenschlossen. Auf diese Weise etablierten sie sich mit der Zeit als erfolgreiche Geschäftsleute mit gu- 
ten nationalen und internationalen Beziehungen. Wirtschaftlicher Erfolg und Netzwerke ermöglichten es ihnen, verstärkt Einfluss in der lokalen oder nationalen Politik auszuüben. Darüber hinaus konsolidierten traditionelle Autoritäten ihre Macht, indem sie auf lokalpolitischer Ebene informell staatliche Aufgaben übernahmen - sowohl anstelle von als auch in Ergänzung zu den Behörden. In Kontexten, in denen staatliche Institutionen schwach oder nicht vorhanden waren, ersetzten traditionelle Autoritäten sie und erwiesen sich darüber als wichtige Dienstleister*innen für die lokale Bevölkerung (Kyed/Buur 2007: 2).

Im Zuge des Paradigmenwechsels internationaler Geberorganisationen hin zu lokalen und dezentralen Verwaltungsstrukturen gewannen traditionelle Autoritäten als Schlüsselakteure wieder zunehmend an politischer Relevanz (Kirst 2019). Die Regierung Ghanas erkannte diese Relevanz an und räumte traditioneller Herrschaft mit der Verfassung von 1992 einen formellen Status ein. Traditionelle und staatliche Herrschaft ist dabei strikt getrennt. Diese Trennung wird unter anderem dadurch deutlich, dass dem Parlament untersagt ist, sich in chieftaincy-Fragen einzumischen. Das Parlament kann keine chiefs ernennen, ihre Anerkennung verweigern oder Gesetze erlassen, die der chieftaincy-Institution schaden (Republic of Ghana 1992: Art. 270). Darüber hinaus garantiert die Verfassung den Fortbestand des National House of Chiefs. In dieses werden jeweils fünf traditionelle Autoritäten aus den zehn Regional Houses of chiefs abgeordnet, um die Interessen der unterschiedlichen traditionellen Gemeinschaften gegenüber dem Staat sowie innerhalb des chieftaincy-Systems zu vertreten. Sie sollen staatliche Akteure zu chieftaincy-Fragen beraten, traditionelles Recht kodifizieren, zur Harmonisierung von staatlichem und traditionellem Recht beitragen und diesbezüglich mögliche Konflikte identifizieren. Des Weiteren sollen sie eine Beurteilung aktueller traditioneller Gepflogenheiten und Bräuche gewährleisten und darauf hinarbeiten, dass sozial nachteilige gewohnheitsrechtliche Normen und Regeln nicht fortbestehen (ebd.: Art. 271, Art. 272). Abgesehen von der beratenden Funktion traditioneller Autoritäten untersagt die Verfassung ihnen, sich aktiv in der Parteipolitik zu engagieren. Traditionellen Autoritäten ist es nicht erlaubt politische Ämter zu bekleiden. Streben sie einen politischen Posten an, müssen sie ihre Position als chief aufgeben (ebd.: Art. 276). Diese Maßnahme soll die politische Einflussnahme auf traditionelle Herrschaft verhindern (Elhadary/Obeng-Odoom 2012).

In Bezug auf die Verwaltung von Land erkennt die Verfassung die $\mathrm{Zu}$ ständigkeit traditioneller Autoritäten an (Republic of Ghana 1992: Art. 267, 
Abs. 1) und schreibt ihnen die Rolle von Treuhändern der jeweiligen Gemeinschaft zu, die sich verpflichten zum Wohl und Nutzen ihrer Gemeinschaft zu handeln (ebd.: Art. 36, Abs. 8). Die Verteilung der Einnahmen aus dem Land überträgt sie dem staatlichen Office of the Administrator of Stool Lands (OASL) (ebd.: Art. 267, Abs. 2). Laut verfassungsrechtlich festgelegtem Verteilungsschema behält dieses zehn Prozent aller Einnahmen zur Deckung administrativer Ausgaben. Die verbleibenden Einnahmen gehen zu 25 Prozent an den Stool, 20 Prozent erhält die traditionelle Autorität in ihrer treuhänderischen Funktion und 55 Prozent die staatliche District Assembly für infrastrukturelle Verbesserungen in der Gegend (ebd.: Art. 267, Abs. 6). Der Stool bezeichnet in diesem Zusammenhang alle Mitglieder der Gemeinschaft ${ }^{1}$.

\section{Rechtspluralismus als Fundament gegenwärtiger Landrechte}

Gegenwärtige Landrechte in Ghana leiten sich aus der National Land Policy von 1999 ab (Ministry of Lands and Forestry 1999). Ziel dieser ersten umfassenden Bodenpolitik Ghanas war es, zentrale Herausforderungen in der Verwaltung von Land herauszuarbeiten und ein kohärentes Landrechtssystem zu entwickeln. Insbesondere hebt die National Land Policy hervor, dass es in der Vergangenheit an Maßnahmen mangelte, über welche die Vergabe von Land erfasst werden könne, um beispielsweise eine Mehrfachvergabe $\mathrm{zu}$ verhindern. Die wichtigste Herausforderung in Bezug auf gewohnheitsrechtlich verwaltetes Land seien unklare Grenzen und ein Mangel an detaillierten Landkarten. Darüber hinaus zählten großflächige Zwangsaneignungen vermeintlich unbewirtschafteter Flächen durch die Regierung, ein schwaches Landverwaltungssystem und konkurrierende Landnutzungsformen $\mathrm{zu}$ den größten Schwierigkeiten (Ministry of Lands and Forestry 1999).

An Gesetzgebungen mangelte es im Landsektor jedoch nicht. Insgesamt identifiziert die National Land Policy 166 unterschiedliche Gesetzgebungen mit Bezug zur Landverwaltung. Aufgrund von Überschneidungen, Widersprüchen und Schwachstellen bildeten diese jedoch kein kohärentes Landrechtssystem (Quaye 2006). Das sollte die National Land Policy ändern, indem sie die »sinnvolle Nutzung des nationalen Lands und all seiner natürlichen Ressourcen von allen Bevölkerungsschichten, die unter Berücksichtigung der Prinzipien des nachhaltigen Ressourcenmanagements unterschiedlichen sozio22.3.2016. 
ökonomischer Aktivitäten nachgehen und dabei funktionsfähige Ökosysteme erhalten, sicherstellt« (Ministry of Lands and Forestry 1999: 6, Übers. d. Autorin).

Um die National Land Policy umzusetzen, startete die Regierung 2003, finanziert durch die Weltbank und andere Geberorganisationen, das Land Adminstration Project (LAP) (World Bank 2003). Das Projekt bestand aus zwei Phasen. Die erste dauerte von 2003 bis 2011 und die zweite zunächst von 2011 bis 2016, wurde dann aber bis Februar 2018 verlängert. Das übergeordnete Ziel des LAP war die Sicherung von Landrechten, insbesondere mittels Registrierung und Formalisierung zuvor informeller Rechte. Entsprechend der neoliberalen Ausrichtung der Maßnahmen, liegt diesem Ziel die Annahme zugrunde, die Sicherung von Landrechten sei eine unabdingbare Voraussetzung von Wachstum, wirtschaftlicher Entwicklung und Armutsminderung sowie einer Zunahme landbezogener Investitionen und einer effizienteren Landnutzung (World Bank 2003). Mit der Sicherung von Landrechten waren institutionelle Reformen verbunden, die dazu dienten, das Landverwaltungssystem zu dezentralisieren. Fortan sollten unabhängig agierende Behörden unter dem Dach der neuen Lands Commission existieren und zu einer besseren Landverwaltung beitragen.

Neben der Harmonisierung von staatlichen und traditionellen Landrechten strebte das LAP insbesondere die Registrierung traditioneller Landrechtsansprüche sowie die Aufzeichnung der Grenzen traditionell verwalteten Lands an. Vor allem in ländlichen Gegenden sind traditionelle Landrechtsansprüche in der Regel nicht dokumentiert (Elhadary/ObengOdoom 2012: 63; Schoneveld/German 2014), was im Rahmen des LAP als ein Hindernis in der erfolgreichen Verwaltung von Land galt. Zur Stärkung der traditionellen Landverwaltung sollte, wie in der National Land Policy formuliert, das LAP lokale Customary Land Secretariats (CLS) unter dem Vorsitz traditioneller Autoritäten aufbauen. Diese sollen zu einer größeren Transparenz in der Verwaltung von gewohnheitsrechtlich genutztem Land beitragen. Aufgabe der CLS ist die Registrierung individueller Landrechtsansprüche sowie die Bearbeitung landbezogener Konflikte. Damit sollen sie eine Schnittstelle zwischen staatlicher und traditioneller Landverwaltung bilden (Schoneveld et al. 2011; Stacey 2015). In der Praxis hat sich jedoch herausgestellt, dass die CLS dazu beitragen, den Einfluss traditioneller Autoritäten in der Verwaltung und Vergabe von Land zu erhöhen. Denn den Vorsitz der CLS haben immer traditionelle Autoritäten inne. Fraglich ist daher, ob sie tatsächlich eine Neuerung darstellen oder nur eine durch den 
Staat geschaffene traditionelle Institution, die den Einfluss traditioneller Autoritäten in Landfragen stärkt. Nach Abschluss des Land Administration Project wurde deutlich, dass im Rahmen seiner Implementierung versäumt wurde zu hinterfragen, inwiefern chiefs in ihrem Handeln kontrolliert werden müssten, um sie daran zu hindern, sich Pachteinnahmen anzueignen, die ansonsten dem Gemeinwohl zustehen würden. Diese Problematik geht auf den Versuch zurück, in einem Zuge die staatliche Landverwaltung und die Verwaltung durch traditionelle Autoritäten zu stärken (World Bank 2013: ix).

Das gegenwärtige Landrecht unterscheidet drei Kategorien von Land: (1) state land, (2) customary land und (3) private land (Kasanga/Kotey 2001). Innerhalb dieser drei Kategorien differenziert es zwischen (1) state land und vested land, (2) stool, skin und family land sowie (3) individual und private land. State land sind Flächen, die sich der Staat im Laufe der Zeit im öffentlichen Interesse und für öffentliche Zwecke wie den Bau von Infrastruktur angeeignet hat. Die gesetzliche Grundlage dafür bildet der State Lands Act von 1962 (Republic of Ghana 1962b). Mit der Aneignung des Lands durch den Staat und nach einer gesetzlich festgelegten Entschädigungszahlung an die ehemaligen Landnutzer*innen erlöschen alle vorherigen Nutzungsrechte (Kasanga/Kotey 2001: 1). Vested land sind Flächen, die einer dualen Eigentumsstruktur unterliegen. Sie werden über den Administration of Lands Act reguliert (Republic of Ghana 1962a). Der Staat erhebt einen rechtlichen Anspruch auf das Land, überlässt die gewohnheitsrechtliche Verwaltung jedoch traditionellen Autoritäten. Der Zugang zu Land und die Nutzungsrechte verbleiben in diesem Zusammenhang in der landbesitzenden Gruppe, während der Staat die Einnahmen aus dem Land treuhänderisch im Sinne der jeweiligen Gemeinschaft verwaltet. Die durch das Land erzielten Einnahmen verteilt die staatliche Lands Commission an die lokale Regierung, traditionelle Autoritäten und Landbesitzer*innen, wobei sie einen Teil zur Deckung der administrativen Kosten einbehält (Amanor 2009: 97f.). Etwa 80 Prozent aller landwirtschaftlichen Flächen in Ghana fallen unter die Kategorie customary land, das traditionelle Autoritäten in traditionellen Landverwaltungssystemen auf der Grundlage von Gewohnheitsrecht verwalten (Kasanga/Kotey 2001: 1).

\section{Traditionelle Landverwaltungssysteme in Ghana}

Im Gegensatz zu formal-staatlichem Recht haben traditionelle Landverwaltungssysteme ihren Ursprung nicht in der staatlichen Politik, sondern beruhen auf verbal übermitteltem Gewohnheitsrecht, das gesellschaftliche Regeln 
und Normen widerspiegelt. Diese Regeln und Normen sind aus der jeweiligen Gemeinschaft heraus entstanden und historisch gewachsen. Sie sind das Ergebnis gesellschaftlicher Aushandlungsprozesse und variieren von Region zu Region und von Dorf zu Dorf. Die Ausgestaltung traditioneller Landverwaltungssysteme unterscheidet sich daher zwischen den Regionen Ghanas. Unterschiede finden sich nicht zuletzt auch in den Bezeichnungen für gewohnheitsrechtlich verwaltetes Land, etwa stool land, skin land, clan oder family land. Die Begriffe leiten sich aus den unterschiedlichen Bräuchen und Herrschaftsinstitutionen ab. Im Süden Ghanas sitzen traditionelle Autoritäten bei ihrer Ernennung zum chief sowie zu offiziellen Anlässen auf hölzernen Schemeln (stools), im Norden auf Tierhäuten (skins), welche die Macht der chiefs symbolisieren. Die Zeremonie der Ernennung eines chiefs wird daher auch als enstoolment oder enskinment bezeichnet ${ }^{2}$. Die Begriffe stool land und skin land gehen auf diesen Brauch zurück. Stool oder skin bezeichnet darüber hinaus die Gesamtheit einer traditionellen Gemeinschaft. Diese umfasst die traditionellen Autoritäten und ihre »Untergebenen« (subjects), die über die Anerkennung traditioneller Autoritäten maßgeblich zu deren Legitimation beitragen ${ }^{3}$.

Gewohnheitsrechtliche Ansprüche auf Land haben in der Regel eine lange Geschichte. In vielen Fällen werden sie über die erste Niederlassung einer Gruppe in einer bestimmten Gegend legitimiert (Berry 2009b). Kennzeichnend für gewohnheitsrechtlich verwaltetes Land ist jedoch die Koexistenz vielfacher, parallel existierender Rechte. Unterschiedliche Personen tragen, abhängig von ihrem Status und ihrer Position innerhalb der Bezugsgruppe, die Verantwortung für die Vergabe dieser Rechte (Berry 1993). Die Nutzungsrechte für gewohnheitsrechtlich verwaltetes Land liegen in der Gemeinschaft, die erfolgreich ihren Anspruch auf das Land geltend macht. Diese Gemeinschaft hat den Allodial Title inne, den höchsten Besitzanspruch im Gewohnheitsrecht, über dem kein übergeordnetes Interesse existiert (Kasanga/Kotey 2001: 13). Bei stool und skin lands repräsentiert der zuständige chief den Allodial Title (Republic of Ghana 1992: Art. 268, Abs. 261) und verwaltet als Treuhänder für seine Gemeinschaft das Land.

In Bezug auf die Verwaltung von Land überträgt er Verantwortung an unter ihm stehende sub-chiefs oder village chiefs (Odikros), die für die Vergabe von

2 Vorsitzender des National Office of the Administrator of Stool Lands, Interview am 22.3.2016.

3 Vorsitzender des National Office of the Administrator of Stool Lands, Interview am 22.3.2016. 
Abbildung 4: Darstellung des durch einen paramount chief verwalteten Lands

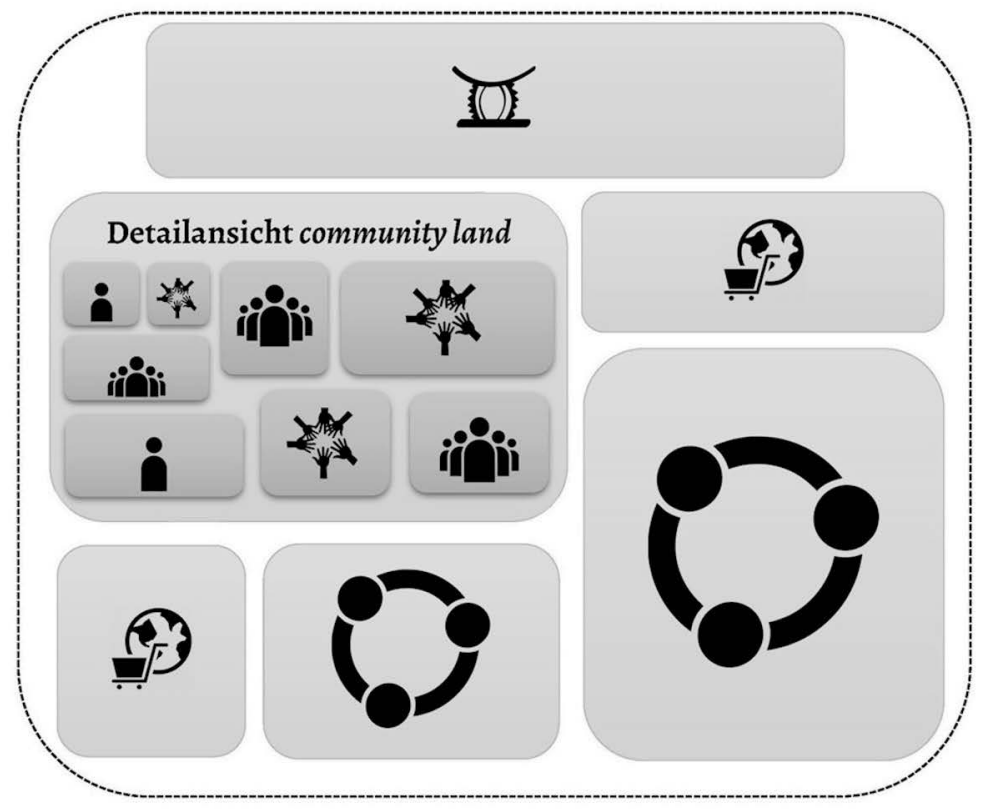

\section{Legende}

Stool land des paramount oder
sub-chiefs

Quelle: Eigene Darstellung

Landnutzungsrechten auf Dorfebene zuständig sind. Alle chiefs können - unabhängig von ihrer hierarchischen Stellung - einen Teil des durch sie verwalteten Lands für sich selbst beanspruchen. In Abgrenzung zu gemeinschaftlich genutztem Land (communal land) wird dieses wiederum als stool oder skin land bezeichnet. Über dieses Land können die chiefs allein verfügen, ohne mit den 
Mitgliedern der Gemeinschaft Rücksprache zu halten. Bei family land oder clan land verfügt das zuständige (erweiterte) Familienoberhaupt über den Allodial Title und damit über das Recht Land zu vergeben. Landnutzungsrechte von Individuen oder Familien, die Teil der landbesitzenden Gruppe sind, beruhen entweder auf dem customary freehold oder dem usufruct. Der customary freehold ist ein dauerhaftes Recht und ermöglicht es neben der eigenen Nutzung, Land an Nachkommen zu vererben und anderen Landnutzer*innen (z.B. im Rahmen von sharecropping-Vereinbarungen oder Pachtverträgen) zur Verfügung zu stellen.

Da die Gemeinschaft immer die Lebenden, Toten und Ungeborenen umfasst, verbietet das Gewohnheitsrecht den Verkauf von Land. Der Verkauf von Land würde bedeuten, die eigene Entscheidung über diejenige der Toten und Ungeborenen zu stellen (Ahmed et al. 2018: 575). Der usufruct ist für Mitglieder der Gemeinschaft ein permanentes Recht. Im Unterschied zum customary freehold erlaubt der usufruct es ihnen jedoch nicht, das Land an Dritte weiterzugeben und ist an die Nutzung des Lands geknüpft. Migrant*innen - Landnutzer*innen, die ursprünglich aus anderen Gegenden oder Ländern kommen - erhalten meist temporäre Nutzungsrechte. Diese sind zudem mit einer geldlichen Gegenleistung oder einer Naturalpacht (sharecropping) verbunden. Jährlich oder nach jeder Ernte sind sie verpflichtet einen Teil der Ernteerzeugnisse an den sub-chief bzw. die Landbesitzer*innen, die das Land zur Verfügung stellen, abzugeben. Gängige sharecropping-Vereinbarungen in Ghana sind abusa und abunu. Abusa bedeutet, dass zwei Drittel der erwirtschafteten Erzeugnisse an den Pächter oder die Pächterin gehen und die Landbesitzer*innen das verbleibende Drittel bekommen. Dafür sind die Pächter*innen selbst für die Vorbereitung des Lands zuständig. Abunu bedeutet, dass Pächter*innen und Landbesitzer*innen die Ernteerzeugnisse gleichmäßig teilen. Unter dieser Vereinbarung tragen Landbesitzer*innen meist gemeinsam mit den Pächter*innen die Verantwortung für die Verwaltung der Farm.

Investoren erhalten Nutzungsrechte an traditionell verwaltetem Land ebenfalls über Pachtvereinbarungen. Verfassungsrechtlich ist die maximale Pachtdauer von Land für Ausländer*innen auf 50 Jahre begrenzt (Republic of Ghana 1992: Art. 266, Abs. 4). Ghanaer*innen hingegen können Land auch für einen längeren Zeitraum pachten. Investoren, die traditionell verwaltetes Land pachten möchten, müssen - unabhängig davon von wem sie das Land pachten - den für die Landverwaltung zuständigen chief persönlich um sein Einverständnis bitten. Um traditionellen Autoritäten ein Anliegen unterbreiten zu können, ist es üblich ihnen zuvor als Zeichen des Respekts 
eine symbolische Gabe $\mathrm{zu}$ überreichen. Traditionell war dies eine Flasche Schnaps, im Norden Ghanas sind es manchmal auch Kolanüsse. Seit einigen Jahren werden Schnaps oder Kolanüsse immer häufiger durch kleine Summen Geld ersetzt, die - unter Rückbezug auf den früheren Brauch - als drink money bekannt sind. Mit dem zunehmenden Interesse von Investoren an der großflächigen Aneignung von Land begannen chiefs große Geldsummen $\mathrm{zu}$ verlangen, um Zugang $\mathrm{zu}$ Land $\mathrm{zu}$ ermöglichen (Blocher 2006; Schoneveld/German 2014). Die Bezeichnung drink money und der damit verbundene Rückbezug auf einen üblichen Brauch rechtfertigt diese Vorgehensweise, an die sich Investoren halten müssen, um erfolgreich mit ihrem Vorhaben zu sein.

Abbildung 5: Traditionelle Landrechte in Ghana

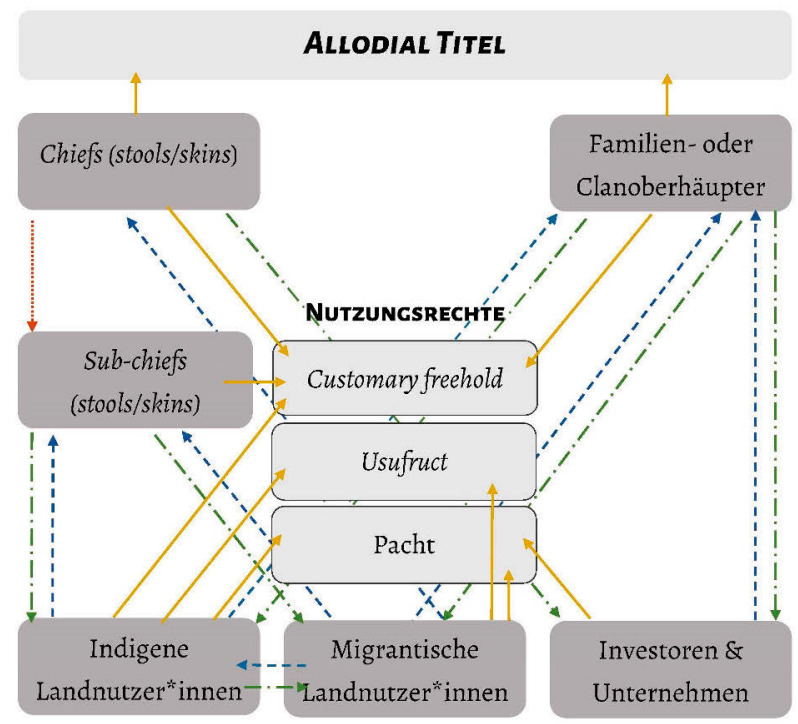

$\underline{\text { Legende }}$

$\ldots$ Übertragen Verantwortung

$\rightarrow$ Legitimieren Rechtsansprüche

$-\rightarrow$ Übertragen Rechte

$\longrightarrow$ Verfügen über

Quelle: Eigene Darstellung 


\subsection{Traditionelle Herrschaft in der Ashanti und Northern Region}

Wie in vielen anderen Ländern Afrikas ist traditionelle Herrschaft in Ghana einer kleinen Gruppe privilegierter Personen vorbehalten. Den Anspruch auf dieses Privileg machen sie geltend, indem sie angeben, patrilineale oder matrilineale Nachkommen einer bestimmten Abstammungslinie (lineage) zu sein. Diese trägt oftmals die Bezeichnung royal family. Dabei handelt es sich um die Abstammungslinie, dessen Vorfahren als Gründer*innen der jeweiligen Gemeinschaft gelten. Traditionelle Gesellschaften unterteilen sich in zwei Sozialgruppen: eine kleine Gruppe »Adeliger« (royals), welche die traditionelle Elite bildet und einen Großteil »Nichtadeliger « (commoners). Ausschließlich Personen aus der traditionellen Elite können den Posten einer traditionellen Autorität erlangen (Tonah 2012: 4).

Das chieftaincy-System in der Ashanti Region ist streng hierarchisch organisiert, mit dem Oberhaupt der Ashanti, dem Asantehene, an der Spitze. hene bedeutet auf Akan König oder chief (Agyekum 2006: 225). Asantehene bedeutet also König der Ashanti. Der Asantehene ist der Inhaber des Golden Stool und hat den Vorsitz des Ashanti Regional House of Chiefs sowie des Kumasi Traditional Council inne. Zwischen dem Tod eines Asantehene und der Wahl seines Nachfolgers vertritt ihn der Mamponghene, der Inhaber des Silver Stool und Vorsitzender des Mampong Traditional Council. Insgesamt umfasst das traditionelle Herrschaftssystem der Ashanti 36 traditional councils, die jeweils für eine bestimmte Gegend (traditional areas) zuständig sind. Einen traditional council bilden ein paramount chief (omanhene) und unter ihm stehende sub-chiefs, auch als elders bezeichnet, sowie queenmothers. Der paramount chief hat den Vorsitz des traditional council. Einige der sub-chiefs stehen den zahlreichen kleineren Gemeinschaften innerhalb einer traditional area vor und sind dem paramount chief zu Loyalität verpflichtet. Andere haben eine beratende Funktion.

In der Northern Region gibt es, anders als in der Ashanti Region, kein einheitliches chieftaincy-System und damit auch nicht nur ein einziges Oberhaupt wie den Asantehene. Zum Zeitpunkt der Feldforschung ${ }^{4}$ gab es in der Northern Region fünf traditional councils, an deren Spitze jeweils ein traditionelles Oberhaupt steht. Der untersuchte Fall befindet sich im Zuständigkeitsbereich 
des Dagomba Traditional Council, dem größten traditional council der Region. Der Dagomba Traditional Council hat seinen Sitz in Yendi und sein traditionelles Oberhaupt ist der Ya Naa (Ahorsu 2014: 100). Naa (oder Na) bedeutet König oder chief in Dagbani, der Sprache der Dagombas (Brukum 2005: 27; Ladouceur 1972: 98). Ya Naa bedeutet king of strength (Iddrisu 2013: 25). Ähnlich wie das Ashanti chieftaincy-System ist das traditionelle Herrschaftssystem der Dagombas hierarchisch organisiert. An der Spitze befindet sich der Ya Naa. Unter ihm stehen paramount und sub-chiefs. Jeder chief ist dem in der Hierarchie über ihm stehenden chief zu Loyalität verpflichtet (Tonah 2012: 6).

\section{Ernennung traditioneller Autoritäten}

Eine Gemeinsamkeit traditioneller Autoritäten in allen Regionen - egal ob paramount chiefs, sub-chiefs oder andere Posten innerhalb der traditionellen Elite - ist, dass sie ernannt und nicht gewählt werden. Die Ernennung ist so genannten kingmakers oder queenmakers vorbehalten, die ebenfalls aus einer royal family abstammen müssen, selbst jedoch niemals Anwärter*innen auf den entsprechenden Posten sein können. Vor der Ernennung beraten sich diese mit anderen elders aus der traditionellen Elite, um eine* $n$ geeignete* $n$ Kandidat*in auszuwählen.

Das chieftaincy-System in der Ashanti Region ist matrilineal, was so viel bedeutet wie »in der Erbfolge der mütterlichen Linie folgend« (Dudenredaktion o.J.). Matrilineal ist nicht mit matriarchal zu verwechseln. Anders als in einem Matriarchat sind hohe Posten in matrilinealen Gemeinschaften nicht zwangsläufig Frauen vorbehalten. Das gilt auch für das chieftaincy-System in der Ashanti Region, in der die chief-Posten, bis auf wenige Ausnahmen, ausschließlich von Männern besetzt werden. Weibliche chiefs können nur ernannt werden, wenn es keinen männlichen Anwärter für den Thron gibt (BoafoArthur 2003: 135). Die Matrilinearität des chieftaincy-Systems in der Ashanti Region verkörpert die Königinmutter (queenmother). Geeignete Kandidaten für den chief-Posten müssen aus ihrer Blutlinie abstammen und sie ist als kingmaker für ihre Nominierung zuständig. Dementsprechend kann sie ihren eigenen Sohn nominieren, wie es auf den aktuellen Asantehene Osei Tutu II zutrifft. Sie kann aber auch den Sohn ihrer Schwester, den Bruder ihrer Mutter oder den Sohn ihrer Tante mütterlicherseits nominieren. Ihre Nominierung muss durch die elders des traditional council abgesegnet werden. Nur aufgrund berechtigter Einwände dürfen diese die Nominierung ablehnen. Lehnen die elders drei Nominierte in Folge ab, dürfen sie selbst einen 
Kandidaten nominieren und ernennen. Diese Prozedur der Ernennung gilt für alle unterschiedlichen chief-Posten in der Ashanti-Region ${ }^{5}$.

Das chieftaincy-System der Dagombas ist patriarchal. Die Ernennung traditioneller Autoritäten berücksichtigt also die väterliche Linie der Erbfolge und erfolgt, wie auch in Ashanti, über die kingmaker. Der Großteil aller chiefs in Dagbon sind Männer. In drei Städten (Kukulogu, Kpatuya, Gundogu) sind jedoch Frauen die chiefs. Diese drei skins sind den Töchtern des Ya Naa vorbehalten (Boafo-Arthur 2003: 135f.). Anders als im chieftaincy-System der Ashanti unterscheidet sich die Ernennung des Ya Naa von derjenigen der unter ihm stehenden traditionellen Autoritäten. Paramount und sub-chiefs müssen, wie auch in Ashanti, jeweils aus einer bestimmten Abstammungslinie kommen, die einem bestimmten skin zugeordnet ist ${ }^{6}$. Einige dieser skins sind so genannte gate skins, die Zugang zu einem höheren skin, also einer höheren Position im chieftaincy-System ermöglichen (Ladouceur 1972: 98). Andere skins bieten diese Möglichkeit nicht. Ihren Inhaber*innen bleibt es verwehrt, in der Hierarchie aufzusteigen. Neben der zentralen Rolle der kingmaker in der Ernennung neuer chiefs, bedarf diese immer auch der Zustimmung des amtierenden Ya Naa sowie höher stehender chiefs (MacGaffey 2006: 89). Die Ernennung zum chief ist damit maßgeblich an gute Beziehungen zur traditionellen Elite geknüpft.

Die Möglichkeit zum Ya Naa ernannt zu werden, besteht ausschließlich für die paramount chiefs der drei gate skins Karaga, Mion und Savelugu. Diese skins sind den Söhnen des $\mathrm{Ya}$ Naa vorbehalten, so dass nur der Sohn eines ehemaligen Ya Naas selbst Ya Naa werden kann. Die Ernennung des Ya Naas erfolgt über ein rotierendes System, in dem sich die drei lineages der gate skins in der Thronfolge ablösen (Ahorsu 2014: 100f.). Eine traditionelle Kommission von vier kingmakers ernennt den neuen $\mathrm{Ya}$ Naa. Ihre Entscheidung beruht auf der Qualifikation des zukünftigen Ya Naas sowie auf Wahrsagen durch ein Orakel (MacGaffey 2006: 88).

\section{Traditionelle Landverwaltung in den Forschungsregionen}

Laut Verfassung obliegt die Verwaltung von Land in beiden Forschungsregionen den jeweiligen stools und skins, die treuhänderisch, im Einklang mit tra-

6 Research Officer des Regional House of Chiefs der Northern Region, Interview am 8.3.2017. 
ditionellen Rechten und im Sinne der Mitglieder der Gemeinschaft darüber verfügen können (Republic of Ghana 1992: \$267, Abs. 1). Die traditionellen Rechte in Bezug auf die Verwaltung von Land in den beiden Forschungsregionen weisen viele Ähnlichkeiten, aber auch einige Unterschiede auf.

Das Land in der Agogo Traditional Area lässt sich in stool land, communal land, family land und individual land unterteilen. Stool land ist immer einem bestimmten stool zugeordnet und steht dem Inhaber des jeweiligen stools also dem paramount chief oder dem sub-chief - zur freien Verfügung. Communal land ist Land, dass der gesamten Gemeinschaft - zu der beispielsweise auch Migrant*innen gehören - zur Verfügung steht. Je nach Bedarf vergibt der sub-chief temporäre Nutzungsrechte für dieses Land. Für Mitglieder der Gemeinschaft sind diese Nutzungsrechte permanent, so lange sie das Land bestellen. Auf family land können diejenigen Familien Anspruch erheben, die glaubhaft machen können, dass ihre Vorfahren die ersten Nutzer*innen des Lands waren. Rechte auf dieses Land sind in der Regel permanent und vererbbar, auch wenn das Land eine Zeitlang brach liegt. Individual lands bezeichnet Flächen, für die Landnutzer*innen verbriefte traditionelle Rechte, also Rechte die durch eine traditionelle Autorität schriftlich bestätigt und nicht nur mündlich festgehalten wurden, erworben haben. Solche Rechte kann nur der paramount chief gewähren und sie sind in der Regel mit einer Gegenleistung, z.B. einer Beteiligung an der Ernte verbunden ${ }^{7}$.

Für die alltägliche Vergabe und Verwaltung von Land auf Dorfebene ist der sub-chief zuständig. Eine Ausnahme bildet family land, das der jeweilige family head, das Oberhaupt der Familie, verwaltet. Für die Vergabe von Land für kommerzielle Zwecke ist auf Dorfebene ebenfalls der sub-chief zuständig, wobei er den paramount chief darüber in Kenntnis setzen und die Einnahmen mit ihm teilen muss. Für Flächen, die über die Dorfebene hinausgehen, ist der paramount chief zuständig ${ }^{8}$. Seine Aufgabe ist es in diesem Fall allerdings, die jeweiligen sub-chiefs darüber zu informieren und sie zu konsultieren. Die Aufgabe der sub-chiefs ist es wiederum, die Informationen an ihre Gemeinschaft

7 Registerführer des Agogo Traditional Council und Leiter des Agogo Customary Land Secretariat, Interview am 20.4.2016; Landnutzer Nsonyameye und Vorsitzender der Agogoman mma kuo, Interview am 16.3.2017.

8 Registerführer des Agogo Traditional Council und Leiter des Agogo Customary Land Secretariat, Interview am 20.4.2016. 
weiterzuleiten und die Meinung der jeweiligen Landnutzer*innen einzuholen ${ }^{9}$.

In Dagbon verfügt, laut traditionellem Recht, der Ya Naa über jegliches skin land (Ahorsu 2014: 101). Er überträgt die Aufgabe der Landverwaltung jedoch an unter ihm stehende paramount chiefs. In der Projektgegend sind das die chiefs von Sang (Sang Lana), Mion (Mion Lana), Salankpang (Salankpang Lana), Zakpalsi (Zakpalsi Lana) und Tijo (Tijo Naa). Die alltägliche Verwaltung von Land für kleinbäuerliche Landwirtschaft übernehmen sub-chiefs, die einzelnen Dörfern vorstehen. Auf Dorfebene können Familienoberhäupter zur Bestreitung ihres Lebensunterhalts für ihre Familie ein Stück Land frei auswählen und müssen nur den sub-chief darüber informieren. Migrant*innen erhalten Zugang zu Land durch den sub-chief, dem sie als Zeichen des Respekts einige Kolanüsse mitbringen. Einmal zugestandene Landnutzungsansprüche können in der Regel weder für Mitglieder der Gemeinschaft noch für Migrant*innen rückgängig gemacht werden. Der Ya Naa oder der paramount chief können jedoch Land im Interesse der Gemeinschaft vergeben. Indigenen, die in diesem Zuge Land verlieren, erhalten einen entsprechenden Ersatz. Migrant"innen haben keinen Anspruch auf Entschädigung (Tsikata/Yaro 2011: 18). Die Vergabe von Land für die kommerzielle Nutzung obliegt den paramount chiefs, die ebenfalls dafür zuständig sind, alle Familienoberhäupter ihrer Gemeinschaft sowie die unter ihnen stehenden sub-chiefs zu informieren. Darüber hinaus muss die Landvergabe durch den $\mathrm{Ya} N a a$ abgesegnet werden. Dabei handelt es sich um einen formellen Akt. Er segnet die entsprechende Landvergabe mit seiner Unterschrift $a b$ und erhält im Gegenzug einen Anteil des Geldes.

Die Verwaltung und Vergabe von Land in den beiden Forschungsregionen weist zum größten Teil Gemeinsamkeiten auf. Ein Unterschied betrifft die Vergabe von Land für kommerzielle Zwecke. In der Ashanti Region kontrollieren die paramount chiefs die Vergabe von Land für kommerzielle Zwecke, indem sie es selbst vergeben oder die Vergabe von durch sub-chiefs verwaltetem Land absegnen und in diesem Zuge Anspruch auf einen Teil der Einnahmen erheben. Paramount chiefs in der Ashanti Region - mit Ausnahme der Kumasi Traditional Area - sind niemandem in der Hierarchie höher stehenden zu Rechenschaft verpflichtet. Paramount chiefs in der Northern Region hingegen sind dem Ya Naa zu Rechenschaft verpflichtet, der die Vergabe von Land am 20.4.2016. 
134 Umkämpfter Zugang zu Land

für kommerzielle Zwecke in letzter Instanz absegnet. Diese Absegnung ist in der Regel eine Formsache und schränkt die paramount chiefs nicht in ihrer Entscheidungsfreiheit ein. 


\section{Empirische Beispiele für Konflikte um Zugang zu Land im Kontext von land grabbing}

In diesem Kapitel stelle ich die empirischen Fälle, die als Grundlage meiner Analyse dienen, dar. Diese Fälle sind die Konflikte um die agrarindustriellen Projekte ScanFarm Ltd in der Ashanti Region und BioFuel Africa Ltd in der Northern Region. Zunächst gebe ich einen Überblick über die Entstehung sowie den Verlauf der Projekte bis Anfang 2017, dem Zeitpunkt meines letzten Feldforschungsaufenthalts. Anschließend gehe ich auf drei Aspekte näher ein. Diese drei Aspekte sind der Ablauf der Landvergabe an die Unternehmen, die mit der Landvergabe verbundenen Konflikte sowie die in die Konflikte involvierten Akteure und ihr Handeln in den Konflikten.

\subsection{Das ScanFarm-Projekt - Landvergabe, Konflikte, Konfliktakteure}

2007 initiierten Thor Hesselberg, der Geschäftsführer von ScanFuel Ghana Ltd und drei weitere norwegische Mitbegründer des Unternehmens gemeinsam mit einem ghanaischen Geschäftspartner die Verhandlungen über einen Pachtvertrag für ein agrarindustrielles Jatropha-Projekt in der Gegend um die Dörfer Dukusen, Nsonyameye und Baamaa. Diese befinden sich im Asante Akim North District in der Ashanti Region unweit der Distrikthauptstadt Agogo. ScanFuel Ghana Ltd. ist ein Tochterunternehmen des im selben Jahr gegründeten norwegischen Konzerns ScanFuel AS Norway. Seine Hauptinvestoren sind der norwegische Unternehmer Stale Kyllingstad sowie das norwegische Investmentunternehmen IKM Invest, das Teil der Unternehmensgruppe IKM ist. Ebenso wie die Gründungsmitglieder kommen die Investoren aus der Öl- und Gasindustrie (Roelf 21.11.2008). Mit dem Ziel, die Energiepflanze Jatropha für die Produktion von Agrarkraftstoff anzubauen, 
Abbildung 6: Karte der Regionen Ghanas und den Projektstandorten von ScanFarm und BioFuel Africa

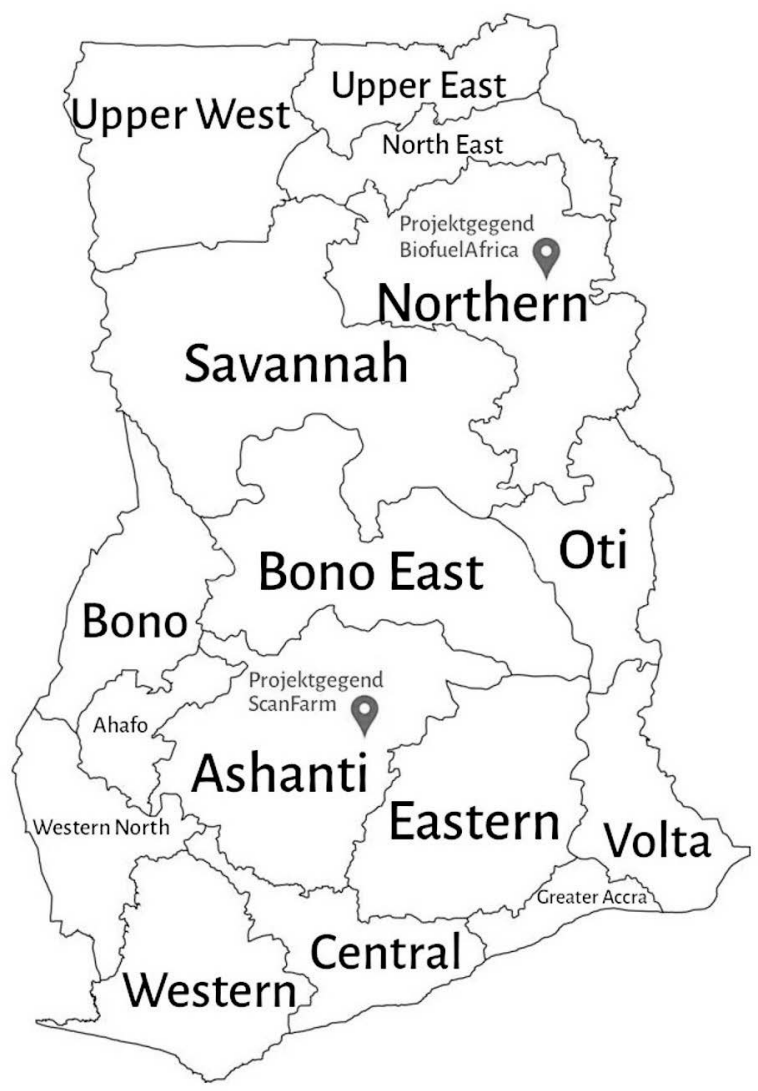

Quelle: Eigene Darstellung

strebte das Unternehmen die Aneignung von etwa 400.000 Hektar Land an. 60 Prozent der Fläche sollten für den Anbau von Jatropha und mindestens 30 Prozent für den Anbau von Nahrungsmitteln genutzt werden. Die verbleibende Fläche sollte als Biodiversitäts-Zone unbewirtschaftet bleiben (Dogbevi 30.08.2010; Roelf 21.11.2008). Im November 2008, auf der Biofuel Markets Africa Conference in Cape Town, Südafrika, berichtete der Geschäftsführer 
des Unternehmens, Thor Hesselberg Vertreter*innen der Presse, ScanFuel habe einen Vertrag über die entsprechende Fläche von 400.000 Hektar abgeschlossen und plane zunächst auf 10.000 Hektar Jatropha anzubauen. Weitere 10.000 Hektar seien für die Produktion von Nahrungsmitteln für den lokalen Markt vorgesehen. Mit der Zeit solle der Anbau ausgeweitet werden. Bis 2015 plane ScanFuel 5000 Barrel Jatropha-Rohöl pro Tag zu produzieren (Roelf 21.11.2008).

Entgegen der Aussage des ScanFuel-Geschäftsführers kam ein Pachtvertrag über die 400.000 Hektar nie zustande. Auch das Vorhaben, Jatropha anzubauen, gab das Unternehmen nach nur einem Jahr auf. Der Rückzug einiger Investoren im Zuge der Finanzkrise, steigende Ölpreise um 2009 und Jatropha-Erträge, die weit hinter dem erwarteten Maß zurückblieben, bewegten das Unternehmen dazu, seine Produktionsstrategie und in diesem Zuge auch seinen Namen zu ändern ${ }^{1}$.

Abbildung 7: Karte der Projektgegend von ScanFarm

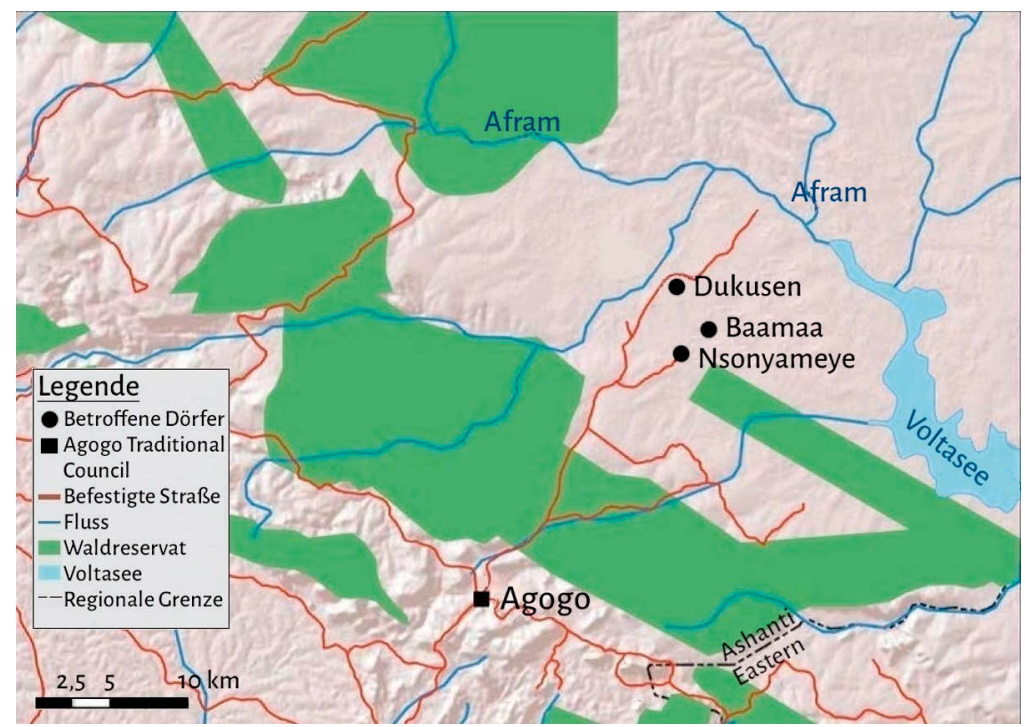

Quelle: Boamah 2014a: 416, eigene Bearbeitung.

1 Ehemaliges Vorstandsmitglied von ScanFarm, Interview am 6.5.2016. 
Seit 2009 ist das Unternehmen unter dem Namen ScanFarm registriert, den ich im weiteren Verlauf des Buches verwende. Seither produziert das Unternehmen vornehmlich Mais ${ }^{2}$. Diesen bezeichnet das ghanaische Landwirtschaftsministerium (Ministry of Food and Agriculture) als priority crop - eine Nutzpflanze, dessen Nachfrage insbesondere als Nahrungsmittel in den letzten Jahren erheblich angestiegen ist und dessen Anbau gefördert werden soll, um eine größere Ernährungssouveränität Ghanas zu ermöglichen ${ }^{3}$. Mais zählt zu den flex crops, ein Terminus der Agrarrohstoffe bezeichnet, die je nach Nachfrage und Marktpreis flexibel als Nahrungsmittel oder für die energetische und industrielle Nutzung weiterverarbeitet werden können (Borras et al. 2016). ScanFarm produziert nach eigenen Angaben ausschließlich für den nationalen Markt, wobei insbesondere Geflügelproduzent"innen und Brauereien die Ernte aufkaufen ${ }^{4}$. 2010, ein Jahr nach der Umstellung auf Mais, erhielt das Unternehmen, das zu diesem Zeitpunkt noch nicht über einen staatlich registrierten Pachtvertrag verfügte, vom ghanaischen Landwirtschaftsministerium die Auszeichnung als National Best Maize Farmer. Seit 2016 ist ScanFarm im Besitz eines staatlich registrierten Pachtvertrags. 2017, zur Zeit meines letzten Feldforschungsaufenthalts, umfasste dieser eine Fläche von 1250 Hektar, wovon das Unternehmen 700 Hektar bewirtschaftete. Ziel des Unternehmens war es, diese Fläche auszuweiten, sobald die gesamten 1250 Hektar für den Anbau genutzt werden ${ }^{5}$.

\section{Landvergabe}

Die Vergabe des Lands in der Gegend von Dukusen, Nsonyameye und Baamaa an ScanFarm geht auf die Initiative von Kyie Bafour zurück, der selbst aus Agogo stammt und bis 2009 Vorstandsmitglied des Unternehmens war. Er hatte die norwegischen Gründungsmitglieder von ScanFarm über riesige Flächen verfügbaren Lands in seiner Heimatgegend informiert und war maßgeblich in die Aneignung des Lands involviert. Zunächst wandte er sich an die paramount chiefs von Gira, Kumawu, Atebubu und Agogo, da das von diesen chiefs verwaltete Land gemeinsame Grenzen hat. Alle vier begrüßten das Vorhaben

\footnotetext{
Geschäftsführer ScanFarm, Interview am 4.4.2016.

Programmleiter Ministry of Food and Agriculture, Interview am 23.3.2016.

Geschäftsführer ScanFarm, Interview am 4.4.2016.

Ceschäftsführer ScanFarm, Interview am 13.3.2017.
} 
und stellten große Flächen dafür in Aussicht ${ }^{6}$. Da sich das Projekt langsam entwickeln sollte, strebte ScanFarm zunächst nur Verhandlungen mit dem Agogo Traditional Council (ATC) an. Dieses Vorhaben wurde maßgeblich dadurch erleichtert, dass Kyie Bafours Onkel der sub-chief von Dukusen - einem der Dörfer in der späteren Projektgegend - war. Dadurch hatte Kyie Bafour direkten Zugang zu traditionellen Institutionen der Landverwaltung. Mit seiner Einschätzung, das Projekt bringe Entwicklung in Form neuer Infrastruktur (wie Straßen, Schulen, Krankenstationen, Wasserdämmen, Brunnen, Elektrizität etc.), sauberer Energie in Zeiten der Energiekrise und des Klimawandels sowie Beschäftigungsmöglichkeiten für die lokale Bevölkerung, konnte er den sub-chief schnell von dem Vorhaben überzeugen ${ }^{7}$. Da dieser nur über sein eigenes stool land frei entscheiden kann und ScanFarm Zugang zu einer Fläche erlangen wollte, die weit über dessen Grenzen hinausging, verwies der subchief von Dukusen das Unternehmen mit seinem Anliegen an den paramount chief. Diesem ist er zu Loyalität verpflichtet, da er derjenige ist der ihn enstooled hat und dem er seine Position zu verdanken hat ${ }^{8}$. Der paramount chief sicherte den Investoren eine Fläche von 19.000 Hektar Land für eine Dauer von 50 Jahren zu. Im Gegenzug soll er laut Aussagen des Geschäftsführers von ScanFarm sowie eines Mitarbeiters des lokalen Landwirtschaftsministeriums eine beträchtliche Summe an drink money erhalten haben ${ }^{9}$. Den genauen Betrag wollten weder der Geschäftsführer des Unternehmens noch die Vertreter des ATC und des CLS nennen. Die zwei ghanaischen Wissenschaftler Elias Danyi Kuusaana und Kaderi Noagah Bukari (2015: 53) sprechen in ihrer Studie zu Konflikten um Land im Asante Akim North District, im Rahmen derer sie auch zu ScanFarm forschten, von 23.000 US-Dollar. 2008 unterzeichneten ScanFarm und der ATC ein Abkommen (memorandum of understanding) ${ }^{10}$. In diesem einigten sich die Parteien auf eine Pachthöhe von einem Dollar pro Hektar und Jahr in 2010, eine jährliche Steigerung von 50 Cent und eine maximale Pachthöhe von 3,50 US-Dollar im Jahr 2014. Darüber hinaus gewährte das Abkommen dem Unternehmen uneingeschränkten Zugang zu jeglichen

$6 \quad$ Ehemaliges Vorstandsmitglied ScanFarm, Interview am 6.5.2016.

7 Sub-chief von Dukusen, Interview am 19.4.2016.

8 Sub-chief von Dukusen, Interview am 19.4.2016.

9 Geschäftsführer ScanFarm, Interview am 4.4.2016; Abteilungsleiter des Regional Office of the Administrator of Stool Lands der Ashanti Region, Interview am 14.4.2016.

10 Geschäftsführer ScanFarm, Interview am 4.4.2016; Registerführer des Agogo Traditional Council und Leiter des Agogo Customary Land Secretariat, Interview am 20.4.2016 
Wasservorkommen auf dem Land und betonte, dass potenzielle Konflikte zunächst durch den ATC bearbeitet werden sollten (Kuusaana/Bukari 2015: 56). Die sub-chiefs und Landnutzer"innen der sich auf dem Land befindenden Dörfer setzte der paramount chief darüber nicht in Kenntnis. Erst nachdem die Investoren und der ATC das Abkommen über die 19.000 Hektar geschlossen hatten, berief der paramount chief die sub-chiefs aus der Gegend zu einem Treffen ein. Im Rahmen dieses Treffens setzte er sie über seine Entscheidung in Kenntnis, sein vermeintlich unbewirtschaftetes stool land - das in offiziellen Dokumenten wie dem Memorandum of Understanding (Absichtserklärung zwischen Unternehmen und Landnutzer*innen) auch als grass land, marginal land oder idle land bezeichnet wird - an ScanFarm zu verpachten. Stool land unterliegt im Gegensatz zu community land der alleinigen Kontrolle durch den Inhaber des jeweiligen stools - in diesem Fall dem paramount chief. Das berechtigt ihn dazu, Verhandlungen über die Vergabe dieses Lands allein zu führen. Die Entscheidung, nur stool land und kein community land zu verpachten, rechtfertigte daher die Entscheidung, die sub-chiefs und Landnutzer*innen zunächst nicht in die Verhandlungen einzubeziehen ${ }^{11}$.

Im Jahr 2008, nachdem ScanFarm und der ATC den Pachtvertrag unterzeichnet hatten, begann das Unternehmen mit der Erlaubnis des paramount chiefs auf 350 Hektar Jatropha anzubauen ${ }^{12}$. Als das Unternehmen 2010 den Anbau von Jatropha beendete, um fortan Mais zu produzieren, wies ein Gutachten darauf hin, dass der Boden in der Gegend sich nicht für das Vorhaben eignete. Daher begann das Unternehmen andere Flächen, die ihm laut Abkommen zustanden, zu erschließen ${ }^{13}$. Dabei stellte sich heraus, dass das durch das Unternehmen genutzte Land nicht wie angekündigt ausschließlich das stool land des paramount chiefs war, sondern auch community land der Dörfer Dukusen, Nsonyameye und Baamaa (Boamah/Overå 2016: 117) umfasste. Der Anbau von Jatropha fand in der Gegend von Dukusen statt. Im Zuge der Umstellung auf Mais begann das Unternehmen auch Grenzen zum community land von Nsonyameye und Baamaa zu überschreiten. Die Landnutzer*innen aus diesen Dörfern realisierten erst nach Beginn der Bearbeitung des Lands durch das Unternehmen, dass ihr Land von dem Projekt betroffen war. Da

\footnotetext{
11 Registerführer des Agogo Traditional Council und Leiter des Agogo Customary Land Secretariat, Interview am 20.4.2016.

12 Geschäftsführer ScanFarm, Interview am 4.4.2016.

13 Geschäftsführer ScanFarm, Interview am 4.4.2016.
} 
es sich bei den Dörfern um farming communities handelt, leben viele der Landnutzer*innen nur in der Anbau- und Erntesaison dauerhaft dort. Die meisten haben ihren permanenten Wohnort in der nahe gelegenen Stadt Agogo. Als sie Kenntnis über die Arbeiten des Unternehmens auf ihrem Land erlangten, waren ihre Felder teilweise schon gepflügt, die Ernte war zerstört, Bäume gefällt und natürliche Grenzmarkierungen entfernt ${ }^{14}$.

Erst nach Beginn der Nutzung des Lands bemühte sich das Unternehmen, den Pachtvertrag bei der Regional Lands Commission registrieren zu lassen und initiierte alle dafür nötigen Schritte wie eine Umwelt- und Sozialverträglichkeitsprüfung (environmental and social impact assessment, ESIA), die Ankündigung des Projekts in den betroffenen Dörfern und ein offizielles Treffen aller Interessengruppen. Aus Unterlagen der Lands Commission geht hervor, dass die 19.000 Hektar nicht nur traditionell verwaltetes Land umfassten. In ihren Untersuchungen stellte die Regional Lands Commission fest, dass ca. 6000 Hektar des Lands bereits auf den ghanaischen Geschäftsmann Bernard Offori registriert waren (Kuusaana/Bukari 2015). In der Konsequenz reduzierte die Lands Commission die Fläche, die für die Registrierung infrage kam, auf 13.000 Hektar (Brief der Lands Commission an ScanFarm, Kumasi, 30.09.2009). In der Zwischenzeit begannen auch einige der Landnutzer"innen und sub-chiefs Einspruch bei dem Unternehmen und der Lands Commission gegen die Vergabe ihres Lands zu erheben. Dabei erhielten sie teilweise Unterstützung durch NGOs wie ActionAid Ghana und das FoodSPAN Netzwerk, die im Rahmen ihrer Advocacy-Arbeit über land grabbing aufklärten und zu Widerstand aufriefen (ActionAid Ghana 2011; Yirrah 2012). Aufgrund des Konflikts verzögerte sich die Registrierung des Pachtvertrags und schließlich lehnte die National Lands Commission, die Pachtverträgen ab einer Größe von 1000 acres $^{15}$ zustimmen muss, sie ab. Als Grund dafür gab die National Lands Commission an, sie erachte die Fläche für das Vorhaben als zu groß. 2016 kam es schließlich doch noch zur Registrierung des Pachtvertrags zwischen ScanFarm und dem ATC. Diese schloss von den ursprünglichen 19.000 Hektar nur 1250 Hektar ein ${ }^{16}$, wobei es sich weiterhin um Land handelt, das zuvor von Kleinbäuerinnen und -bauern genutzt wurde.

Landnutzer*innen aus Nsonyameye, Baama und Dukusen, Interviews am 24., 25., 26. und 27.4.2016. 


\section{Konflikte}

Der Konflikt um das ScanFarm-Projekt entstand, als einige Landnutzer*innen realisierten, dass das Unternehmen ihr Land ohne ihre Zustimmung nutzte.

Um die Angelegenheit zu klären, wandten sich die Landnutzer*innen an die jeweiligen sub-chiefs, die auf Dorfebene für die Schlichtung von Konflikten zuständig sind. Die sub-chiefs, die über die Vergabe des vermeintlichen stool lands an ScanFuel informiert waren, gingen zunächst davon aus, dem Ganzen liege ein Missverständnis zugrunde und das Unternehmen habe versehentlich die Grenzen zu ihrem Land übertreten ${ }^{17}$. Sie berichteten daher dem paramount chief darüber, der beteuerte, er habe kein community land an ScanFarm vergeben. Um dies zu bestätigen schickte der paramount chief ein Mitglied des Agogo Traditional Council (ATC) gemeinsam mit den sub-chiefs auf das Land. Dabei stellte sich heraus, dass sich das Unternehmen tatsächlich zu einem Teil des community land Zugang verschafft hatte. Ob es sich dabei um ein Versehen des Unternehmens handelte oder ob der paramount chief doch mehr als nur das ihr zum freien Verfügung stehende stool land vergeben hatte - was er weiterhin bestritt - blieb zunächst unklar. Deutlich wurde jedoch, dass er keine Ambitionen hatte, das Unternehmen daran zu hindern, weiterhin auf dem Land der Landnutzer*innen zu arbeiten. Entgegen der Bestrebungen der Landnutzer*innen und sub-chiefs, ihr Land zurückzugewinnen, plädierte er für die weitere Nutzung des Lands durch ScanFarm. Er argumentierte, dies sei im Sinne aller, da das Projekt Entwicklung und Arbeit für die Gemeinschaft bringe $^{18}$. Er riet den sub-chiefs, Entschädigungszahlungen mit dem Unternehmen auszuhandeln, statt ihr Land zurückzufordern ${ }^{19}$. Obwohl der paramount chief das Land offiziell vergeben hatte, unterstützte er die sub-chiefs und Landnutzer*innen bei diesem Vorhaben nicht. Die sub-chiefs von Dukusen, Nsonyameye und Baamaa nahmen daraufhin selbst Kontakt zu dem Unternehmen auf, um Entschädigungszahlungen einzufordern ${ }^{20}$. Da ScanFarm im Zuge der Landnutzung Grenzmarkierungen wie Bäume, Sträucher oder Steine zerstört

Sub-chief von Dukusen, Interview am 19.4.2016, sub-chief von Nsonyameye, Interview am 20.04.2016, sub-chief von Baama, Interview am 20.04.2016.

18 Sub-chief von Dukusen, Interview am 19.4.2016.

19 Sub-chief von Dukusen, Interview am 19.4.2016, sub-chief von Nsonyameye, Interview am 20.4.2016.

20 Sub-chief von Nsonyameye, Interview am 20.4.2016; sub-chief von Baama, Interview am 20.4.2016. 
oder entfernt hatte, war es für die Landnutzer*innen schwierig, ihr Land zu lokalisieren ${ }^{21}$. Um Entschädigungszahlungen fordern zu können, müssen Landnutzer*innen jedoch in der Lage sein in der Anwesenheit von Zeug"innen zu zeigen, welches Stück Land sie zuvor genutzt haben. Auch die Ernte war durch die Arbeiten des Unternehmens zerstört worden. Daher konnten die Landnutzer*innen nicht nachweisen, dass sie das Land zuvor bestellt hatten und für den Verlust der Ernte keine Entschädigungszahlungen fordern ${ }^{22}$.

Im Zentrum des Konflikts standen die intransparente Landvergabe sowie der Verlust der Lebensgrundlage. Mit der Intransparenz der Landvergabe sind die mangelnde Information und Konsultierung sowie die fehlenden Partizipationsmöglichkeiten der Landnutzer*innen und sub-chiefs in Bezug auf die Vergabe des Lands gemeint. Zum Verlust der Lebensgrundlage zählen die Zerstörung von Grenzmarkierungen und Ernte sowie unzulängliche Entschädigungszahlungen.

\section{Konfliktakteure}

Die zentralen Akteure in dem Konflikt um Zugang zu dem durch ScanFarm angeeigneten Land können in drei Akteursgruppen unterteilt werden. Ihre Gemeinsamkeit ist, dass sie Ansprüche auf Zugang zu dem umstrittenen Land erheben, wobei sich ihre Interessen diesbezüglich unterscheiden. Diese drei Akteursgruppen sind das Unternehmen ScanFarm, traditionelle Autoritäten und die Landnutzer*innen. Die Unterteilung der Konfliktakteure in diese drei Gruppen dient der Beschreibung des Konflikts. In der Analyse werde ich zeigen, dass traditionelle Autoritäten und Landnutzer"innen in dem Konflikt nicht als kohärente Gruppe handeln, sondern sich durch Heterogenität auszeichnen und unterschiedliche Interessen verfolgen. Zwischen dem Unternehmen, traditionellen Autoritäten und Landnutzer*innen besteht daher eine Vielzahl unterschiedlicher Konflikte und Allianzen.

Die Gruppe der direkt in den Konflikt involvierten traditionellen Autoritäten umfasst den paramount chief, die sub-chiefs der Dörfer Nsonyameye, Dukusen und Baamaa sowie den ehemaligen sub-chief von Dukusen, der den Pos-

Landnutzerin aus Nsonyameye, Interview am 24.4.2016; Landnutzerin aus Nsonyameye, Interview am 26.4.2016; Landnutzerin aus Nsonyameye, Interview am 28.4.2016; Landnutzerin aus Dukusen, Interview am 16.3.2017; Landnutzer aus Nsonyameye und Vorsitzender der Agogoman mma kuo, Interview am 16.3.2017. 
ten des Informanten innehat. Die sub-chiefs sind für die Vergabe von Land an die Landnutzer*innen der Gemeinschaft zuständig. Sie sind zum Teil selbst Landnutzer*innen und vertraut mit den Begebenheiten vor Ort. Der paramount chief ist für die Verwaltung des Lands zuständig, hat aber selbst keinen direkten Bezug dazu. Das bedeutet, dass er nicht in die Vergabe von Land an Landnutzer*innen involviert ist und nicht mit der Nutzung des Lands vor Ort vertraut ist. Für die kommerzielle Nutzung von Land bedarf es jedoch seines Einverständnisses und er erhebt Anspruch auf einen Teil der Einnahmen daraus $^{23}$. Die ghanaische Verfassung gesteht traditionellen Autoritäten die Rolle von Treuhändern zu, jedoch bezeichnet sich der paramount chief selbst als Eigentümer des Landes (owner of the land) und viele Landnutzer*innen erkennen ihn als solchen $a^{24}$. Seine sub-chiefs bezeichnet der paramount chief als Verwalter (caretaker), die nach seinem Willen handeln müssen ${ }^{25}$ Der ehemalige sub-chief von Dukusen ist der so genannte Informant des paramount chiefs. Er informiert den paramount chief über aktuelle Ereignisse in den Dörfern und war dafür zuständig, ScanFarm das Land zu zeigen (sub-chief aus Nsonyameye, Interview am 20.4.2016).

Die Heterogenität innerhalb der Gruppe der Landnutzer*innen drückt sich in Unterschieden hinsichtlich des Zugangs zu, der Rechte an und der Nutzung von Land aus. Rechte an Land unterscheiden sich zwischen indigenen und migrantischen Landnutzer*innen. Die indigenen Landnutzer*innen des von ScanFarm angeeigneten Lands verfügen über family land, über permanente, mündlich durch den sub-chief gewährte Nutzungsrechte (usufruct) oder über permanente, schriftlich durch den paramount chief bestätigte Nutzungsrechte $^{26}$. Migrantische Landnutzer*innen haben temporäre Nutzungsrechte oder Zugang zu Land über sharecropping-Vereinbarungen mit indigenen Landnutzer*innen oder traditionellen Autoritäten ${ }^{27}$. Jenseits bestehender Rechte an Land unterscheiden sich die Landnutzer*innen dadurch, dass einige ihr

23 Registerführer des Agogo Traditional Council und Leiter des Agogo Customary Land Secretariat, Interview am 20.4.2016.

25 Landnutzer aus Dukusen, Interview am 26.3.2016; Mitarbeiter des Asantehenes Land Secretariat, Interview am 10.3.2017.

26 Landnutzerin aus Nsonyameye, Interview am 24.4.2016; Landnutzerin aus Nsonyameye, Interview am 25.4.2016; Landnutzer aus Nsonyameye und Mitglieder der Agogoman mma kuo, Interview am 16.3.2017; Landnutzer aus Nsonyameye und Vorsitzender der Agogoman mma kuo, Interview am 16.3.2017.

27 Landnutzer*innen aus Nsonyameye, Interviews am 24., 25., 27. und 28.4.2016. 
Land vor der Aneignung durch ScanFarm aktiv selbst nutzten, während andere Einkünfte über das Land erzielten, indem sie es an andere Landnutzer*innen verpachteten oder sharecropping-Vereinbarungen eingingen. Auch die Größe des Lands, zu dem die unterschiedlichen Landnutzer*innen vor der Aneignung des Lands durch ScanFarm Zugang hatten, unterscheidet sich. Interviewpartner*innen berichteten von 4 bis 4000 acres, was einem Äquivalent von 1,6 bis 1619 Hektar entspricht ${ }^{28}$. Dieses Land nutzten sie selbst oder ihre Pächter*innen für den Anbau von Bananen, Papaya, Mango, Melone, Mais, Yams, Maniok, Zwiebeln, Tomaten, Erbsen und Erdnüssen. Außerdem befanden sich auf dem Land Shea- und Johannisbrotbäume, die der Herstellung von Sheabutter und Dawadawa, einem in Westafrika weitverbreiteten fermentierten Gewürz, dienen. Je nach Größe des Lands diente es der reinen Subsistenzwirtschaft oder ermöglichte den Verkauf eines Anteils der Erzeugnisse. Außerhalb der Anbau- und Erntesaison gingen viele der Landnutzer*innen in Agogo Tätigkeiten außerhalb der Landwirtschaft nach, unter anderem als Schneider*innen, Schuster*innen, Schreiner oder Kleinhändler*innen ${ }^{29}$.

Die soziale Differenzierung der Landnutzer*innen und traditionellen $\mathrm{Au}$ toritäten spiegelt sich auch in ihren Reaktionen auf den Verlust des Zugangs zu Land wider. Migrantische Landnutzer*innen, die Zugang zu ihrem Land verloren, schimpften zwar auf das Unternehmen, verließen das Land jedoch, ohne Widerstand zu leisten. »Als Fremder hatte ich nicht viel zu sagen « ${ }^{30}$, erklärte ein migrantischer Landnutzer aus dem Norden Ghanas, dessen Familie seit zwei Generationen in der Gegend lebt ${ }^{31}$. Indigene Landnutzer*innen mit permanenten, nicht formalisierten Rechten an Land erkundigten oder beschwerten sich zunächst bei dem jeweiligen sub-chief, der die Beschwerden an den paramount chief weiterleitete. Als sich daraufhin nichts an der Situation änderte, machten sie Gebrauch von konfrontativeren Strategien. Einige weigerten sich ihr Land zu verlassen und drohten mit dem Einsatz von

28 Landnutzer*innen aus Nsonyameye und Dukusen, Interviews am 24., 25, 26., 27. und 28.4. 2016 sowie am 16.3.2017; sub-chief von Nsonyameye, Interview am 20.4.2017.

29 Landnutzer*innen aus Nsonyameye, Interviews am 24., 25., und 27.4.2016; Landnutzerin aus Dukusen, Interview am 16.3.2017; Landnutzer aus Nsonyameye und Vorsitzender der Agogoman mma kuo, Interview am 16.3.2107; sub-chief von Dukusen, Interview am 19.4.2016.

30 Dieses Zitat sowie alle nachfolgenden Zitate aus Interviews sind von mir aus dem Englischen übersetzt.

31 Landnutzer aus Nsonyameye, Interview am 25.4.2016. 
Waffen $^{32}$. Andere nahmen an Demonstrationen teil, um ihr Land zurückzufordern ${ }^{33}$. Im April und Juni 2010 demonstrierten zahlreiche Landnutzer*innen und Bewohner*innen von Agogo gegen land grabbing und verschafften sich Zugang zum Palast des paramount chiefs (Boamah 2015; Kuusaana 2016). Schließlich forderten zahlreiche Landnutzer*innen gemeinsam mit den subchiefs von Nsonyameye und Baamaa Entschädigungszahlungen von ScanFarm für den Verlust ihres Lands und die Zerstörung der Ernte. Diese Gruppe agierte jedoch nicht als geschlossene Einheit. Einige akzeptieren das erste Angebot des Unternehmens und nahmen darüber hinaus Tätigkeiten an, die das Unternehmen ihnen anbot. Andere, zu denen auch die sub-chiefs gehörten, gaben sich nicht damit zufrieden und forderten höhere Entschädigungszahlungen $^{34}$. Wieder andere gaben die Verhandlung mit ScanFarm auf, nachdem sie feststellten, wie wenig Entschädigung das Unternehmen ihnen für das Land zahlen wollte. Dies verdeutlich auch die Aussage eines indigenen Landnutzers aus Nsonyameye: "Als mir bewusst wurde, dass die Entschädigungszahlungen gering waren, beschloss ich, meine Zeit nicht zu verschwenden. Seitdem bin ich nie wieder zum Unternehmen gegangen, da ich es für nutzlos halte « ${ }^{35}$. Landnutzer*innen mit formalisierten, also schriftlich durch den paramount chief anerkannten, Landrechten konfrontierten zunächst den paramount chief mit dem Verlust ihres Lands an ScanFarm. Der paramount chief bestätigte diese Rechte, unternahm jedoch nichts, um das Unternehmen an der Nutzung des Lands zu hindern. Nachdem das Unternehmen den Forderungen der Landnutzer*innen, ihnen ihr Land zurückzugeben, nicht nachkam, erhoben einige von ihnen 2014 über das staatliche Rechtssystem Klage gegen das Unternehmen ${ }^{36}$. Einige von ihnen waren Mitglieder des zivilgesellschaftlichen Netzwerks Agogoman mma kuo (weltweite Bürger*innen von Agogo), die sich für gesellschaftspolitische Belange der Bürger*innen von Agogo einsetzt. Die Agogoman mma kuo engagierten sich ebenfalls für Entschädigungszahlungen,

32 Landnutzer aus Nsonyameye und Vorsitzender der Agogoman mma kuo, Interview am 16.3.2017; Geschäftsführer ScanFarm, Interview am 4.42016.

33 Landnutzer aus Nsonyameye, Interview am 28.4.2016; Landnutzer aus Nsonyameye und Vorsitzender der Agogoman mma kuo, Interview am 16.3.2017.

34 Sub-chief von Nsonyameye, Interview am 20.4.2016; sub-chief von Baamaa, Interview am 20.4.2016.

35 Landnutzer aus Nsonyameye, Interview am 28.4.2016.

36 Landnutzer aus Nsonyameye und Mitglieder der Agogoman mma kuo, Interview am 16.3.2017. 
mehr Arbeitsplätze für Menschen aus der Gegend und eine größere Transparenz der Landvergabe, beispielsweise durch Einsicht in das Abkommen zwischen dem paramount chief und dem Unternehmen ${ }^{37}$. Darüber hinaus unterbreiteten sie dem Asantehene, dem traditionellen Oberhaupt der Ashanti, eine Petition zur Amtsenthebung (destoolment) des paramount chiefs von Agogo Nana Akuo Sarpong. Als Grund für die Petition führten sie an, er sei »kläglich an der Aufgabe gescheitert, das Land, für das die Vorfahren gekämpft und welches sie hinterlassen haben zu schützen sowie die Bürgerinnen und Bürger jederzeit $\mathrm{zu}$ beschützen und zu verteidigen $^{38}$. Dieses Vorhaben blieb jedoch erfolglos.

\subsection{Das BioFuel Africa-Projekt - Landvergabe, Konflikte und Konfliktakteure}

BioFuel Africa Ltd ist eine Tochtergesellschaft des norwegischen Unternehmens BioFuel Norway und seit 2007 in Ghana registriert (Bruce 2008). Ziel der Gründer von BioFuel Africa war es, in der Northern Region Ghanas die weltweit größte Jatropha-Plantage aufzubauen (Nyari 2008). Die Hauptinvestoren waren das finnische Staatsunternehmen Neste Oil und der norwegische Gasund Ölproduzent STATOIL (seit 2018 Equinor) (Nygaard/Bolwig 2018). Um geeignetes Land dafür zu finden, begannen sie 2005 in ghanaischen Zeitungen $\mathrm{zu}$ inserieren. Auf diese Weise entstand der Kontakt zu ihrem späteren ghanaischen Geschäftspartner, Herrn Awal. Dieser unterstützte die norwegischen Gründer von BioFuel Africa dabei, Land im damaligen Yendi District (heute Mion District) und im Central Gonja District der Northern Region für den Anbau von Jatropha zu identifizieren und den Kontakt zu den traditionellen Autoritäten, die das Land verwalteten, herzustellen. Vorteilhaft dafür war, dass er der Enkelsohn des Tijo Naa (chief von Tijo) ist, der später für die Vergabe des Lands an BioFuel Africa verantwortlich war. Seit 2007 ist BioFuel Africa als Unternehmen in Ghana registriert und erlangte laut unterschiedlichen Quellen sowie Aussagen des Geschäftsführers von BioFuel Africa (Kol-

\footnotetext{
37 Landnutzer aus Nsonyameye und Vorsitzender der Agogoman mma kuo, Interview am 16.3.2017.

38 Landnutzer aus Nsonyameye und Mitglieder Agogoman mma kuo, Interview am 16.3.2017; Landnutzer aus Nsonyameye und Vorsitzender der Agogoman mma kuo, Interview am 16.3.2017; Auszug aus der Petition, Übersetzung durch die Autorin)
} 
nes 2009) Zugang zu insgesamt 23.760 Hektar Land in den beiden Distrikten. 2008 erhielt es nach der Durchführung einer Umwelt- und Sozialverträglichkeitsprüfung für die gesamte Fläche eine Genehmigung (environmental permit) durch die ghanaische Umweltbehörde (Environmental Protection Agency, EPA) (Boamah 2011b). Seit 2009 sind 10.696 Hektar im Mion District bei der Lands Commission registriert (Kolnes 2009). Das Vorhaben im Central Gonja District hingegen gab BioFuel Africa aufgrund von Widerstand seitens der Bevölkerung schnell auf und konzentrierte sich ausschließlich auf das Land im Mion District (Tsikata/Yaro 2011). Das Unternehmen gibt an, insgesamt einen Pachtvertrag über 154.778 Hektar abgeschlossen zu haben (Kolnes 2009; SGS Environment 2009). In einigen Presseberichten und Publikationen von NGOs ist demgegenüber von einer Flächengröße von 38.000 Hektar die Rede (Knaup 05.09.2008; Nyari 2008). Unklar bleibt, woher diese Daten stammen. Zusätzlich zu dem Land in der Northern Region verfügt das Unternehmen über 840 Hektar Land in der Volta Region nahe Sogakope. Diese Fläche nutzte es als Testfarm für den Jatropha-Anbau (SGS Environment 2009).

Abbildung 8: Karte der betroffenen Dörfer des BioFuel Africa-Projekts

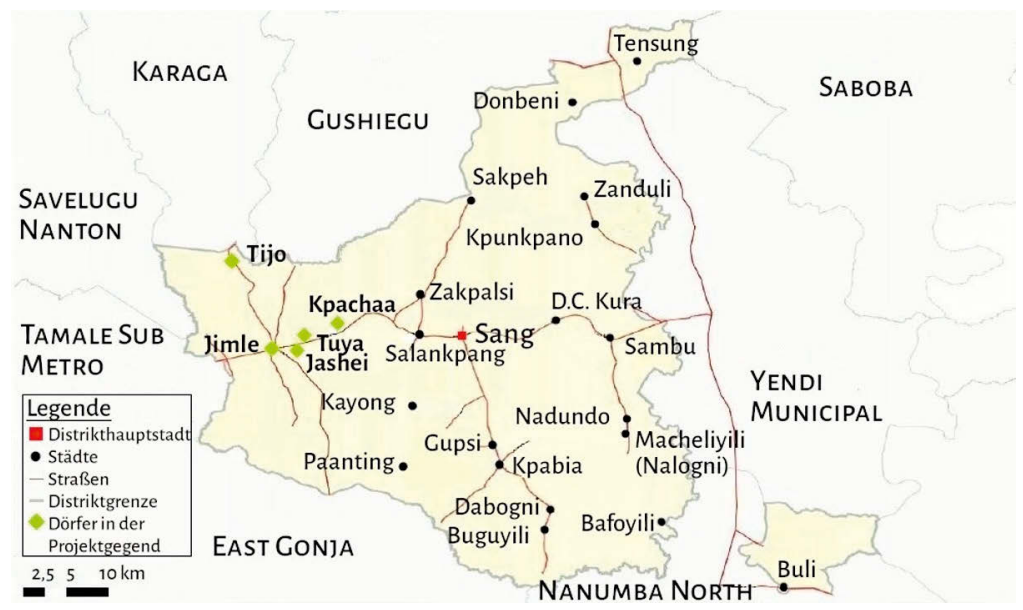

Quelle: Ghana Statistial Service 2014: 2. Eigene Darstellung

Im Jahr 2007 begann BioFuel Africa in der Gegend um die Dörfer Kpachaa, Jashei, Jimle und Tuya auf einer Fläche von 480 Hektar mit dem JatrophaAnbau (SGS Environment 2009). Während der Hochsaison beschäftigte das 
Unternehmen 320 Mitarbeiter*innen, von denen zwei Drittel aus Tamale und ein Drittel aus den Dörfern in der Projektgegend kamen (Bruce 2008). BioFuel Africa strebte an, diese Fläche bis 2011 auf 17.000 Hektar auszuweiten (Kolnes 2009). Dazu kam es jedoch nicht. Vielmehr sorgte das Projekt 2008 nationale und international für negative Schlagzeilen. Grund dafür war die Entscheidung des Unternehmens, ohne Genehmigung durch die Lands Commission und die Umweltbehörde sowie ohne Konsultierung der Landnutzer*innen zu beginnen, Felder für die agrarindustrielle Bewirtschaftung vorzubereiten. Dabei wurden zahlreiche Bäume gefällt, die für die Landnutzer*innen von hohem wirtschaftlichem Wert waren. Dies nahmen insbesondere die NGO Action Aid Ghana und die zivilgesellschaftliche Organisation Regional Advisory and Information Network Systems (RAINS) zum Anlass, um gegen das Projekt zu mobilisieren und zu breitem Widerstand gegen land grabbing aufzurufen (Nyari 2008; Yirrah 2012).

Dennoch produzierte das Unternehmen Ende 2008 das erste noch unkommerzielle Jatropha-Rohöl, ungereinigtes Pflanzenöl, wie es nach der Ölpressung vorliegt (Kolnes 2009). Aufgrund der negativen Presse, bestehender Korruptionsvorwürfe sowie steigender Ölpreise entzog der Gas- und ÖlGigant STATOIL kurze Zeit später, im Jahr 2009, dem Projekt die zugesicherten finanziellen Mittel (Tsikata/Yaro 2011: 21). Aufgrund der fehlenden Finanzierung musste das Unternehmen im März 2009 Konkurs anmelden (persönliche Korrespondenz, Geschäftsführer BioFuel Africa, 15.02.2017). BioFuel Africa baute somit nur zwei Jahre lang Jatropha auf dem Land $a^{39} .2009$ kauften zwei der ehemaligen Gründungsmitglieder, Steinar Kolnes und Arne Helvig, BioFuel Africa auf, mit dem Ziel, fortan Nahrungsmittel zu produzieren. 2010 benannten sie das Unternehmen in Solar Harvest um (Boamah 2011a; Tsikata/Yaro 2011). Seit 2014 erhält Solar Harvest finanzielle Förderung durch das Ghana Commercial Agriculture Project (GCAP) des ghanaischen Landwirtschaftsministeriums. Mithilfe der Förderung will das Unternehmen in Botanga (Kunbungu District, Northern Region) auf 200 Hektar Land ein Bewässerungssystem aufbauen, das den Anbau von Reis, Mais und Soja im Rahmen von Vertragslandwirtschaft ermöglichen soll (SAL 2017). GCAP ist ein durch USAID und die Weltbank finanziertes Projekt zur Förderung einer kommerziellen Landwirtschaft. 


\section{Landvergabe}

Für die Vergabe des Lands an BioFuel Africa spielten insbesondere der ghanaische Geschäftspartner von BioFuel Africa, Adam Awal, der Tijo Naa und sein Anwalt sowie der Regent of Dagbon (in Vertretung des $\mathrm{Ya} \mathrm{Naa}$ ) eine zentrale Rolle. Auf der Suche nach einer geeigneten Fläche für den Anbau von Jatropha wandte sich der ghanaische Geschäftspartner von BioFuel Africa gemeinsam mit den norwegischen Investoren zunächst an den sub-chief von Jimle, seinem Heimatort und überzeugte ihn von dem Vorhaben ${ }^{40}$. Dieser verwies ihn an den Tijo Naa - der vom Status her mit einem paramount chief zu vergleichen ist. Einige elders aus Jimle begleiteten Adam Awal zum Tijo Naa, der bestätigte, dass es sich um seinen Enkelsohn handelte, was für die Gemeinschaft nur Gutes bedeuten könne, und befürwortete die Vergabe von Land (Tsikata/Yaro 2011).

BioFuel Africa initiierte daraufhin eine Veranstaltung im Tamale Cultural Center mit allen zuständigen chiefs, einigen der betroffenen Landnutzer* innen, Angestellten der District Assembly und einigen NGOs, um über das Projekt zu informieren. Sie stellten Busse zur Verfügung, die die Teilnehmenden aus den unterschiedlichen Dörfern in die ca. $50 \mathrm{~km}$ entfernte Regionalhauptstadt Tamale brachten. Im Rahmen dieser Veranstaltung machte der Tijo Naa deutlich, dass er sich von dem Projekt in erster Linie erhoffe, dass es der Gemeinschaft zugutekomme ${ }^{41}$. Im Anschluss an das Treffen besichtigte BioFuel Africa das zur Verfügung stehende Land und engagierte das ConsultingUnternehmen SGS Ghana, um eine Umwelt- und Sozialverträglichkeitsprüfung (ESIA) durchzuführen (SGS Environment 2009). Darüber hinaus organisierte das Unternehmen zwei weitere öffentliche Anhörungen in Tijo und Yendi, wo der Regent of Dagbon seinen Sitz hat. Dieser stimmte der Vergabe des Lands durch den Tijo Naa zu, woraufhin der Tijo Naa, der selbst Analphabet ist, einen Anwalt beauftragte, um den Pachtvertrag mit dem Unternehmen aufzusetzen. Der Pachtvertrag umfasste eine Fläche von 13.800 Hektar, von denen später 10.696 Hektar bei der Lands Commission registriert wurden. Er war auf 25 Jahre begrenzt, jedoch mit Aussicht auf eine Verlängerung von weiteren 25 Jahren nach Ablauf der Frist (Tsikata/Yaro 2011). Die Pacht betrug zwei Ghanaische Cedi (GHS) pro Hektar, was zum damaligen Zeitpunkt

40 Landnutzer Gimle und Gumani, Gruppendiskussion am 10.5.2016.

41 Paramount-chief und elders von Tijo, Gruppendiskussion am 8.5.2016. 
ungefähr einem Wert von knapp einem Euro bzw. etwas mehr als einem USDollar entsprach. Die Pachthöhe ist damit ähnlich wie auch in anderen Fällen großflächiger Landvergabe an internationale Unternehmen. Das zeigt der Vergleich mit anderen Studien, die Projekte in diesem Zeitraum untersucht haben (Kuusaana/Bukari 2015: 56). Die erste Zahlung war für die ersten zwei Jahre angesetzt. Darauf sollten jährliche Zahlungen folgen ${ }^{42}$. 40 Prozent der Pacht gingen an den Regent of Dagbon in Vertretung des Ya Naa. Darüber hinaus gab der Tijo Naa den anderen paramount chiefs aus der Gegend sowie seinen eigenen sub-chiefs zwischen 500 und 1000 GHS, was zum damaligen Zeitpunkt etwa 290 bis 480 Euro entsprach. Die finanzielle Teilhabe der sub-chiefs war unabhängig davon, wessen Land der Pachtvertrag umfasste (Tsikata/Yaro 2011).

Den in der Gegend lebenden Landnutzer*innen, deren Land durch das Projekt betroffen war, bot das Unternehmen drei Optionen an. Sie hatten die Möglichkeit ein anderes Stück Land auszuwählen, das Arbeiter*innen des Unternehmens maschinell für sie roden und pflügen würden oder als Arbeiter*innen für BioFuel Africa zu arbeiten, um ihren Lebensunterhalt zu verdienen. Wenn keine dieser beiden Optionen für sie infrage käme, würde das Unternehmen sie in bar für die Nutzung des Landes bezahlen ${ }^{43}$ (Tsikata/Yaro 2011: 19).

\section{Konflikte}

Anders als im Fall von ScanFarm versuchte BioFuel Africa mit Beginn der Verhandlungen gleichermaßen chiefs und betroffene Landnutzer*innen in den Prozess der Landvergabe einzubeziehen, um Konflikte zu vermeiden. Im Rahmen mehrerer öffentlicher Anhörungen erklärte BioFuel Africa ihr Vorhaben gegenüber Repräsentant"innen der betroffenen Dörfer. Um den Nutzen des Projekts für die Gemeinschaft deutlich zu machen, stellte das Unternehmen Arbeitsplätze und die Verbesserung der Infrastruktur in der Gegend in Aussicht ${ }^{44}$. Während des Höhepunkts der Aktivitäten beschäftigte BioFuel Afri$c a$ insgesamt 116 Arbeiter*innen (67 Männer und 39 Frauen) aus der Projektgegend. Der Großteil der qualifizierten Arbeiter*innen (insgesamt ungefähr

\footnotetext{
42 Paramount-chief und elders von Tijo, Gruppendiskussion am 8.5.2016.

43 Landnutzer*innen und sub-chief von Tuya, Gruppendiskussion am 14.5.2016.

44 Paramount-chief und elders von Tijo, Gruppendiskussion am 8.5.2016.
} 
280) kam jedoch aus Tamale (Bruce 2008: 3). Darüber hinaus errichtete BioFuel Africa eine Mühle in der Gegend von Kpachaa, zwei Dämme in der Nähe von Jashei und Jimle und ebnete die Zubringerstraße nach Jimle. Während der Regenzeit pflügten Arbeiter"innen des Unternehmens für einige Landnutzer*innen Land in Jashei ${ }^{45}$ (ebd.: 4). Trotz der Bemühungen um einen transparenten Ablauf der Landvergabe waren nicht alle Landnutzer*innen und sub-chiefs gleichermaßen informiert ${ }^{46}$ (Ahmed et al. 2018: 576). Auch hatte das Projekt nicht für alle die gleichen positiven und negativen Auswirkungen $^{47}$.

Der Konflikt, der sich im Laufe der Implementierung des Projekts herausbildete, betrifft die Intransparenz in der Kommunikation zwischen den einzelnen chiefs sowie zwischen dem Unternehmen und den Bewohner*innen der einzelnen Dörfer, die Rodung wirtschaftlich genutzter Bäume und die Verschlechterung der Lebensgrundlage der Landnutzer*innen, enttäuschte Erwartungen sowie die Veränderung des Lebensraums.

In Jimle, Jashei, Tuya und Changolinaaya waren die Landnutzer*innen und auch der sub-chief schlecht über das Vorgehen von BioFuel Africa bezüglich der Landnutzung informiert ${ }^{48}$. Außer in Jashei und Changolinaayawaren die Landnutzer*innen in den einzelnen Dörfern zwar über das Projekt in Kenntnis gesetzt worden, wurden im weiteren Verlauf jedoch nicht mehr regelmäßig informiert ${ }^{49}$. Sie erhielten keine Informationen darüber, wann das Projekt starten sollte und auch vom Ende des Jatropha-Anbaus erfuhren sie erst, als die Entscheidung bereits getroffen war. Der Mangel an Informationen ging teilweise auf die unzulängliche Kommunikation zwischen den un-

45 Landnutzer*innen aus Jimle und Gumani, Gruppendiskussion am 10.5.2016; Landnutzer*innen und settler-chief aus Kpachaa, Gruppendiskussion am 11.5.2016; Landnutzer*innen und sub-chief aus Jashei, Gruppendiskussion am 15.5.2016.

46 Landnutzer aus Changolinaaya, Interview am 12.5.2016; Landnutzer und settler-chief aus Parachanayilli, Gruppendiskussion am 13.5.2016; Landnutzer*innen und sub-chief aus Tuya, Gruppendiskussion am 14.5.2016; Landnutzer*innen und sub-chief Jashei, Gruppendiskussion am 15.5.2016.

47 Landnutzer*innen und sub-chief aus Jashei, Gruppendiskussion am 15.5.2016.

48 Landnutzer*innen aus Jimle und Cumani, Gruppendiskussion am 10.5.2016; Landnutzer*innen und sub-chief aus Jashei, Gruppendiskussion am 15.5.201.; Landnutzer*innen und sub-chief aus Tuya, Gruppendiskussion am 14.5.2016; Landnutzer aus Changolinaaya, Interview am 12.5.2016.

49 Landnutzer*innen und sub-chief aus Jashei, Gruppendiskussion am 15.5.2016; Landnutzer aus Changolinaaya, Interview am 12.5.2016. 
terschiedlichen chiefs und teilweise auf fehlende Benachrichtigung durch das Unternehmen zurück ${ }^{50}$ (Bruce 2008).

Im Umfeld der Dörfer Tuya, Jashei, Kpachaa und Parachanayilli rodete BioFuel Africa hunderte Bäume, um die Felder für den Jatropha-Anbau anzulegen ${ }^{51}$. Dabei handelte es sich unter anderem um Sheanuss-Bäume und Johannisbrotbäume, deren Früchte Frauen zu Sheabutter und Dawadawa weiterverarbeiteten. Über den Verkauf der Produkte erwirtschafteten sie zusätzliches Einkommen für die Familie. Die Rodung der Bäume schränkte diese Möglichkeit ein. Die Lebensgrundlage der betroffenen Landnutzer*innen verschlechterte sich zusätzlich, da zuvor anwesende Landwirt*innen aus Tamale aufgrund des BioFuel Africa-Projekts Zugang zu dem von ihnen genutzten Land verloren und die Gegend verlassen mussten. Sie hatten es den Anwohner*innen gestattet, die nach der Ernte auf den Feldern verbleibenden Reste zu sammeln, was für diese eine Möglichkeit darstellte, die eigene Ernte oder knappe Essensvorräte aufzustocken ${ }^{52}$.

Im Zentrum des Konflikts stand darüber hinaus die Veränderung des Lebensraums, die mit der Umsetzung des BioFuel Africa-Projekts einherging. Anwohner*innen der Dörfer berichteten, dass durch die Rodung zahlreicher Bäume starker Wind größere Schäden an den Häusern anrichtete als zuvor ${ }^{53}$ und heftige Regenfälle sowie Dürre stärkere Auswirkungen hätten ${ }^{54}$. Zudem wiesen einige Anwohner*innen darauf hin, dass die Umgebung aufgrund der buschigen Jatropha-Pflanzen - die auch über das Projektende hinweg auf dem Land verblieben, da BioFuel Africa sie nicht entfernte - insbesondere für Kinder gefährlich sei, da sich dort viele Schlangen einnisteten ${ }^{55}$.

50 Landnutzer*innen und sub-chief aus Tuya, Gruppendiskussion am 14.5.2016; Landnutzer*innen und sub-chief aus Jashei, Gruppendiskussion am 15.5.2016.

51 Landnutzer*innen und settler-chief aus Kpachaa, Gruppendiskussion am 11.5.2016; Landnutzer und settler-chief aus Parachanayilli, Gruppendiskussion am 13.5.2016; Landnutzer*innen und sub-chief aus Tuya, Gruppendiskussion am 14.5.2016; Landnutzer*innen und sub-chief aus Jashei, Gruppendiskussion am 15.5.2016.

52 Landnutzer*innen und sub-chief aus Tuya, Gruppendiskussion am 14.5.2016.

53 Landnutzer*innen und settler-chief aus Kpachaa, Gruppendiskussion am 11.5.2016; Landnutzer"innen und sub-chief aus Tuya, Gruppendiskussion am 14.5.2016.

54 Landnutzer und settler-chief aus Parachanayilli, Gruppendiskussion am 13.5.2016.

55 Landnutzer ${ }^{*}$ innen und settler-chief aus Kpachaa, Gruppendiskussion am 11.5.2016. 
Schließlich sind die enttäuschten Erwartungen, die viele Landnutzer*innen an das Projekt hatten relevant für den Konflikt ${ }^{56}$. Auch wenn Landnutzer*innen aus allen betroffenen Dörfern positive Auswirkungen des Projekts wie den verbesserten Zugang zu Wasser oder Arbeitsmöglichkeiten beschreiben, kritisieren viele, dass das Projekt nicht allen Landnutzer*innen gleichermaßen zugutegekommen sei. Nur wenige Personen aus den Dörfern seien eingestellt worden. Insbesondere Frauen, die als Arbeiter*innen für das Projekt unterrepräsentiert waren und ältere Männer, die keine Söhne hatten, die für das Unternehmen arbeiteten, gaben an, sich benachteiligt zu fühlen (Bruce 2008). Weit verbreitet war in den Dörfern außerdem die Meinung, nicht ausreichend an dem Geld, welches das Unternehmen für das Land gezahlte hatte, teilzuhaben ${ }^{57}$.

\section{Konfliktakteure}

Die zentralen Akteure in dem Konflikt um Zugang zu dem durch BioFuel Africa anageeigneten Land können, wie auch im Fall von ScanFarm, in drei Akteursgruppen unterteilt werden. Diese drei Akteursgruppen sind das Unternehmen BioFuel Africa, traditionelle Autoritäten und die Landnutzer*innen. Wie auch im Konflikt um ScanFarm sind traditionelle Autoritäten und Landnutzer*innen keine homogenen Gruppen, sondern sozial differenziert. Unterschiede innerhalb der Gruppen bestehen insbesondere in Bezug auf den Zugang zu Land.

Die Gruppe der traditionellen Autoritäten besteht aus dem Tijo Naa, den sub-chiefs und den settler-chiefs. Der Tijo Naa verwaltet das Land in der Projektgegend. Unter ihm stehen die sub-chiefs, an die der Tijo Naa die Zuständigkeit für die Verwaltung von Teilen seines Lands überträgt. Darüber hinaus gibt es in der Gegend einige settler chiefs, die so genannten settler communities vorstehen. In settler communities leben migrantische Landnutzer*innen, das heißt Landnutzer*innen, die ursprünglich aus einer anderen Gegend kommen. Bei ihrer Ankunft in der Gegend baten sie den zuständigen chief um Land. In der

56 Paramount-chief und elders von Tijo, Gruppendiskussion am 8.5.2016; Landnutzer und settler-chief aus Parachanayilli, Gruppendiskussion am 13.5.2016; Landnutzer*innen und sub-chief von Tuya, Gruppendiskussion am 14.5.2016.

57 Landnutzer*innen aus Nsonyameye, Baama \& Dukusen, Gruppendiskussion am 27.4.2016; Landnutzer*innen und sub-chief von Jashei, Gruppendiskussion am 15.5.2016.6. 
Gegend von BioFuel Africa sind die settler communities Kpachaa, Parachanayilli und Changolinaaya ${ }^{58}$. Der chief von Kpachaa hat das Land für seine Gemeinschaft über den Tijo Naa erhalten, diejenigen in Parachanayilli und Changolinaaya über den Jashei-Naa. Settler chiefs vergeben das Land an die Landnutzer*innen in ihrer Gemeinschaft, haben aber keine permanenten Rechte an Land und müssen sich den Entscheidungen der über ihnen stehenden chiefs beugen $^{59}$.

Die Gruppe der Landnutzer*innen in der Gegend des BioFuel AfricaProjekts besteht aus dort ansässigen Kleinbäuerinnen und -bauern sowie aus Landwirt*innen, die das Land zu kommerziellen Zwecken nutzen, jedoch nicht dort leben. Die Kleinbäuerinnen und -bauern aus Jimle, Jashei und Tuya stammen ursprünglich aus der Gegend, bezeichnen sich selbst als indigen und erheben permanente Ansprüche auf ihr Land ${ }^{60}$. Diejenigen aus Kpachaa, Parachanayilli und Changolinaaya stammen ursprünglich aus anderen Gegenden und akzeptieren, dass ihre Rechte an Land temporär sind und sie keinerlei Entscheidungsbefugnis hinsichtlich der Nutzung des Lands haben ${ }^{61}$. Die Gegend des BioFuel Africa-Projekts ist extrem strukturschwach, mit weit auseinanderliegenden, durch unbefestigte Straßen verbundenen Dörfern von meist wenigen Hundert Einwohner*innen. Die Haupteinkommensquelle der Menschen aus der Gegend des BioFuel Africa-Projekts ist die kleinbäuerliche Landwirtschaft. Vorwiegend Männer bauen Mais, Hirse, Bohnen, Maniok, Erdnüsse, Sojabohnen, Okra und Yamswurzeln für den Eigenbedarf und teilweise auch für den Verkauf $a^{62}$. Da die Anbausaison aufgrund der geringen Regenmengen und der kurz andauernden Regenfälle

58 Landnutzer aus Changolinaaya, Interview am 12.5.2016; Landnutzer*innen und settlerchief aus Kpachaa, Gruppendiskussion am 11.5.2016; Landnutzer und settler-chief aus Parachanayilli, Gruppendiskussion am 13.5.2016

59 Landnutzer"innen und settler-chief aus Kpachaa, Cruppendiskussion am 11.5.2016; Landnutzer und settler-chief aus Parachanayilli, Gruppendiskussion am 13.5.2016.

60 Landnutzer ${ }^{*}$ innen aus Jimle und Gumani, Gruppendiskussion am 10.5.2016; Landnutzer"innen und sub-chief von Tuya, Gruppendiskussion am 14.5.2016; Landnutzer“innen und sub-chief von Jashei, Gruppendiskussion am 15.5.2016.

61 Landnutzer aus Changolinaaya, Interview am 12.5.2016; Landnutzer"innen und settlerchief aus Kpachaa, Gruppendiskussion am 11.5.2016; Landnutzer und settler-chief aus Parachanayilli, Gruppendiskussion am 13.5.2016.

62 Paramount-chief und elders von Tijo, Gruppendiskussion am 8.5.2016; Landnutzer*innen aus Nsonyameye, Baama \& Dukusen, Cruppendiskussion am 27.4.2016; Landnutzer*innen aus Jimle und Cumani, Gruppendiskussion am 10.5.2016; Landnutzer*innen und sub-chief von Tuya, Gruppendiskussion am 14.5.2016. 
zeitlich begrenzt ist, sind die Landnutzer*innen neben der Landwirtschaft auf andere wirtschaftliche Tätigkeiten angewiesen. Außerhalb der Anbauund Erntezeit produzieren sie Holzkohle und betreiben Viehzucht. Die Frauen stellen hauptsächlich Sheabutter und Dawadawa her ${ }^{63}$. Mit dem Verkauf von Tieren, Holzkohle, Sheabutter und Dawadawa überbrücken sie maßgeblich den so genannten hunger gap, die Zeit zwischen dem Aufbrauchen der eigenen Erzeugnisse und dem Beginn der nächsten Ernte (Bruce 2008: 11). Landwirt*innen, die vor Beginn des Projekts dem kommerziellen Anbau von Nutzpflanzen nachgingen, haben ihr Land über den Tijo Naa erhalten. Für die Nutzung des Lands mussten sie einen Teil ihrer Ernte an den chief zahlen. Ebenso wie migrantische Landnutzer*innen haben diese Landwirt*innen keine permanenten Rechte an Land.

Die Reaktionen der Konfliktakteure auf die Aneignung des Lands durch BioFuel Africa unterscheiden sich insbesondere zwischen den in der Gegend lebenden Landnutzer*innen und sub-chiefs sowie den Landwirt*innen, die dort kommerzielle Landwirtschaft betrieben, jedoch aus Tamale kamen. Letzteren kann nach traditionellem Landrecht der Zugang zu dem Land jederzeit und ohne Ersatz verwehrt werden, wenn das Land für andere Zwecke, die dem Wohl der Gemeinschaft dienen, benötigt wird. Das machte der Tijo Naa gegenüber dem Unternehmen deutlich und warnte davor, Entschädigungszahlungen an diese Landnutzer*innen zu leisten (Tsikata/Yaro 2011: 21). Einige von ihnen verließen die Gegend und ließen sich an einem anderen Ort nieder, um dort weiterhin Landwirtschaft zu betreiben. Andere gaben ihre Tätigkeit in der Landwirtschaft auf. Ein Teil von ihnen mobilisierte über Zeitungsartikel (Nyari 2008; The New Humanitarian 2009) und Radiosendungen gegen das Unternehmen und erlangte die Aufmerksamkeit nationaler sowie internationaler NGOs, welche die Proteste unterstützten (Tsikata/Yaro 2011: 21). Die Landnutzer*innen in den Dörfern, unabhängig davon ob sie Indigene oder Migrant*innen waren, tauschten sich untereinander über die Missstände aus, überließen weitere Schritte jedoch den jeweiligen sub-chiefs. Die Landnutzer*innen und sub-chiefs der settler communities waren sich bewusst, dass

63 Landnutzer*innen aus Jimle und Gumani, Gruppendiskussion am 10.5.2016; Landnutzer*innen und settler-chief aus Kpachaa, Gruppendiskussion am 11.5.2016; Landnutzer und settler-chief aus Parachanayilli, Gruppendiskussion am 13.5.2016; Landnutzer*innen und sub-chief von Tuya, Gruppendiskussion am 14.5.2016; Landnutzer*innen und sub-chief von Jashei, Gruppendiskussion am 15.5.2016. 
sie nichts gegen die Nutzung des Lands durch das Unternehmen tun konnten. Aussagen wie »Es hat uns geärgert, dass das Unternehmen unser Land genommen hat, aber wir konnten nichts dagegen sagen ${ }^{64}$ oder »Wir haben nicht gekämpft, da wir settler farmer sind und das Land uns nicht gehört « ${ }^{65}$ verdeutlichen das. Die anderen sub-chiefs wandten sich mit ihren Beschwerden an den Tijo Naa. Sie machten auf ihren Unmut und die wahrgenommenen Missstände aufmerksam und bekamen Verständnis entgegengebracht. An der Situation änderte sich dadurch jedoch nichts.

64 Landnutzer und settler-chief aus Parachanayilli, Gruppendiskussion am 13.5.2016.

65 Landnutzer*innen und settler-chief aus Kpachaa, Gruppendiskussion am 11.5.2016. 



\section{Veränderung von Zugang zu Land im Kontext von land grabbing}

In der Analyse meiner Fälle geht es mir um eine theoriegeleitete, verstehende Interpretation der erhobenen Daten mit dem Ziel, empirisch begründete Erklärungsansätze zum Zusammenhang des Handels traditioneller Autoritäten und Konflikten um Zugang zu Land zu entwickeln. Der Ausgangspunkt der Analyse ist die Annahme, dass Konflikte im Kontext von land grabbing eng mit Veränderungen der Kontrolle des Zugangs zu Land sowie der Möglichkeiten, Zugang zu Land zu erlangen, verknüpft sind. Zugang zu Land zu haben bedeutet, einen Nutzen aus Land ziehen zu können. Kontrolle über den Zugang $\mathrm{zu}$ Land ausüben zu können bedeutet, bestimmen zu können, ob und welche Akteure einen Nutzen aus Land ziehen können. Entsprechend erlangen Akteure Zugang zu Land, indem sie sich die Kontrolle darüber aneignen oder indem sie Vereinbarungen mit anderen Akteuren treffen, die Zugang zu Land kontrollieren.

Für die Analyse von Konflikten um Zugang ist es zentral, die einzelnen Mechanismen, über die unterschiedliche Akteure Zugang zu Land erlangen und kontrollieren, zu identifizieren. Aufgrund meines Forschungsfokus auf Konflikte im Kontext der großflächigen Vergabe von Land an Investoren gilt es anschließend herauszuarbeiten, inwiefern sich die Möglichkeiten auf die zuvor identifizierten Mechanismen zurückzugreifen in diesem Zusammenhang verändern. Diesen Fragen gehe ich mit Bezug auf die Konflikte um die agrarindustriellen Projekte ScanFarm und BioFuel Africa im Folgenden nach. Zunächst stelle ich dar, wie die Kontrolle von Zugang zu Land vor der Vergabe des Lands an die Unternehmen ausgestaltet war und über welche Mechanismen Landnutzer*innen Zugang zu Land erlangen konnten. Anschließend zeige ich, wie sich Kontrolle über Zugang zu Land sowie die Möglichkeiten Zugang zu Land zu erlangen im Kontext der großflächigen Landvergabe verändert haben. 


\subsection{Kontrolle über Zugang zu Land vor der Landvergabe}

In beiden Fallregionen sind nach traditionellem Recht die paramount chiefs pro forma die Verwalter des Lands. Landnutzer*innen, sub-chiefs und staatliche Akteure bezeichnen sie gemeinhin auch als Eigentümer des Lands (owner of the land) ${ }^{1}$. Das bedeutet jedoch nicht, dass die paramount chiefs vor der Vergabe des Lands an ScanFarm und BioFuel Africa die alleinige Kontrolle über den Zugang zu Land ausübten.

Paramount chiefs übertragen unter Rückbezug auf Gewohnheitsrecht in beiden Fällen die Verwaltung des Lands - und damit auch teilweise die Kontrolle des Zugangs zu Land - an ihnen untergebene sub-chiefs, die aus der Abstammungslinie der Gründer*innen der in der Gegend lebenden Gemeinschaft (royal family) abstammen. In der Regel verwalten sub-chiefs Land in der Gegend, in der sie selbst auch leben ${ }^{2}$. Die Zuständigkeit für die Verwaltung des Lands erhalten sie im Rahmen ihrer Amtseinführung durch den paramount chief. Fortan kontrollieren sub-chiefs in ihrem Verwaltungsbereich den Zugang zu Land für die subsistenzwirtschaftliche Nutzung durch Mitglieder der Gemeinschaft oder Migrant*innen. Das durch sub-chiefs verwaltete Land besteht aus stool land und community land. Stool land sind Flächen, auf die der traditional council, dem alle chiefs einer traditionell verwalteten Gegend (traditional area) angehören, gewohnheitsrechtlichen Anspruch erhebt. Community land bezeichnet Flächen, die zur gemeinschaftlichen oder individuellen Nutzung durch Mitglieder der Gemeinschaft oder Migrant"innen zur Verfügung stehen. Sub-chiefs kontrollieren Zugang zu stool land und community Land, indem sie Nutzungsrechte für subsistenzwirtschaftliche $Z$ wecke vergeben. Diese Nutzungsrechte können sie Landnutzer"innen aus der Gemeinschaft, die

$1 \quad$ Landnutzer*innen aus Jimle und Cumani, Gruppendiskussion am 10.5.2016; Landnutzer und settler-chief aus Parachanayilli, Gruppendiskussion am 13.5.2016; Landnutzer*innen und sub-chief von Tuya, Gruppendiskussion am 14.5.2016; Landnutzerin aus Nsonyameye, Interview am 25.4.2016; Landnutzer aus Dukusen, Interview am 26.4.2016; Mitarbeiterin des Regional Office of the Administrator of Stool Lands der Northern Region, Interview am 4.5.2016; Research Officer des Regional House of Chiefs der Northern Region, Interview am 8.3.2017; Leitender Angestellter BioFuel Africa, Interview am 24.5.2016. am 19.4.2016; Research Officer des Regional House of Chiefs der Northern Region, Interview am 8.3.2017. 
mehr Land benötigen als ihnen bisher zur Verfügung stand oder Migrant*innen zusprechen ${ }^{3}$. Entsprechend haben die sub-chiefs Kenntnisse darüber, welche Flächen in ihrem Verwaltungsbereich wie und durch wen genutzt werden. Den Zugang zu Land für die Subsistenzwirtschaft kontrollieren sub-chiefs somit unabhängig vom paramount chief. Dieser übt hingegen weiterhin Kontrolle über den Zugang zu Land für kommerzielle landwirtschaftliche Vorhaben aus. Dabei kann es sich um großflächige agrarindustrielle Projekte oder um kleinere Vorhaben handeln, die eine deutliche kommerzielle Ausrichtung haben und durch Akteure umgesetzt werden, die nicht in der jeweiligen Gemeinschaft leben. Der paramount chief ist für die endgültige Bewilligung dieser Vorhaben zuständig und erhebt Einnahmen für die Nutzung des Lands, wie Pacht oder seltener Ernteabgaben. Dem Gewohnheitsrecht entsprechend verteilt er einen Teil dieser Einnahmen an die sub-chiefs, in deren Gegend sich das Vorhaben befindet ${ }^{4}$. Der sub-chief wiederum handelt in diesem Kontext als Berater des paramount chiefs und informiert ihn darüber, welches Stück Land für ein entsprechendes Vorhaben zur Verfügung steht ${ }^{5}$. Darüber hinaus ist der paramount chief in der Gegend von ScanFarm auch für die Formalisierung von Landrechten zuständig, die er gemeinsam mit dem sub-chief bewilligt ${ }^{6}$.

Auf Grundlage des Gewohnheitsrechts kontrollieren neben den sub-chiefs in beiden Fallregionen auch teilweise indigene Landnutzer*innen Zugang zu Land. Dies sind Landnutzer*innen, die über permanente traditionelle Landrechte verfügen. Diese Rechte haben sie nicht über zuständige traditionelle Autoritäten, sondern aufgrund ihrer sozialen Zugehörigkeit erlangt. Nach traditionellem Recht bestehen sie ohne zeitliche Begrenzung und können an Dritte temporär vergeben, dauerhaft übertragen oder vererbt werden. Inhaber*innen solcher permanenten traditionellen Landrechte kontrollierten vor der Vergabe des Lands an die Unternehmen ihren eigenen Zugang sowie den Zugang anderer im Rahmen individueller Absprachen wie sharecropping-oder Pachtvereinbarungen.

Sub-chief von Dukusen, Interview am 19.4.2016; Research Officer des Regional House of Chiefs der Northern Region, Interview am 8.3.2017.

4 Registerführer des Agogo Traditional Council, Interview am 14.3.2017.

5 Sub-chief von Nsonyameye, Interview am 20.4.2016; Research Officer des Regional House of Chiefs der Northern Region, Interview am 8.3.2017.

6 Landnutzer aus Nsonyameye und Vorsitzender derAgogoman mma kuo, Interview am 16.3.2017. 
Auch wenn staatliche Akteure, sub-chiefs und Landnutzer*innen die paramount chiefs in beiden Gegenden als Eigentümer des Lands bezeichnen, übten sie vor der Vergabe des Lands an ScanFarm und BioFuel Africa nur begrenzt Kontrolle über den Zugang zu Land aus. Den Zugang für die subsistenzwirtschaftliche Nutzung kontrollierten de facto sub-chiefs sowie Landnutzer*innen mit permanenten traditionellen Landrechten unabhängig von den paramount chiefs. Sie konnten im Kontext der Subsistenzwirtschaft ohne Rücksprache mit den paramount chiefs ihren eigenen Zugang zu Land und denjenigen anderer Akteure bestimmen. Die paramount chiefs kontrollierten Zugang zu Land nur für die kommerzielle Nutzung von Land und - in der Gegend von ScanFarm - im Rahmen der Formalisierung von Landrechten ${ }^{7}$. In beiden Kontexten agierten sie insbesondere als bewilligende Instanz.

\subsection{Zugang zu Land vor der Landvergabe an ScanFarm und BioFuel Africa}

Die Mechanismen, die es Landnutzer*innen in den Gegenden von ScanFarm und BioFuel Africa vor der Vergabe des Lands an die Unternehmen ermöglichten, Zugang zu dem dortigen traditionell verwalteten Land zu erhalten, sind vielfältig. In erster Linie bestimmten rechtebasierte Mechanismen wie traditionelle Rechte oder individuelle Vereinbarungen den Zugang zu Land. Rechtebasierte Mechanismen ermöglichten jedoch den Zugang zu Land nicht allein, sondern im Zusammenspiel mit relationalen und strukturellen Mechanismen. Relevante relationale Mechanismen waren soziale Zugehörigkeit, soziale Beziehungen sowie Netzwerke. Über diese konnte - innerhalb struktureller Grenzen und damit einhergehenden Ungleichheitsverhältnissen - rechtebasierter Zugang zu Land erlangt oder verhandelt werden. Insbesondere die Interpretation und Aushandlung sozialer Zugehörigkeit innerhalb sozialer Beziehungen war in beiden Fällen relevant, um bestehende Landrechtsansprüche zu konsolidieren oder zu erheben. Rechtebasierter Zugang konnte darüber hinaus innerhalb von Beziehungen ausgehandelt werden, indem Rechteinhaber*innen individuelle Vereinbarungen mit Land-

7 Paramount-chief und elders von Tijo, Gruppendiskussion am 8.5.2016; sub-chief von Nsonyameye, Interview am 20.4.2016; Landnutzer aus Nsonyameye und Vorsitzender der Agogoman mma kuo, Interview am 16.3.2017. 
nutzer*innen ohne bestehende Rechte trafen, um letzteren gegen Pacht oder Naturalleistungen Zugang zu Land zu gewähren.

Im Folgenden stelle ich entlang der rechtebasierten, relationalen und strukturellen Zugangsmechanismen die Ausgestaltung des Zugangs zu Land in den Regionen der beiden untersuchten Fälle vor der Landvergabe an die Unternehmen dar. Daran anschließend analysiere ich, inwiefern sich Zugang $\mathrm{zu}$ Land im Kontext der Vergabe des Lands an ScanFarm und BioFuel Africa verändert hat.

\section{Zugang zu Land über rechtebasierte Mechanismen}

Rechtebasierte Mechanismen, die Landnutzer*innen in den Fallregionen vor der Vergabe des Lands an die Unternehmen Zugang zu Land ermöglichten, waren divers und unterschieden sich zwischen den Landnutzer*innen aufgrund strukturell begründeter Ungleichheitsverhältnisse. Rechte variierten insbesondere zwischen indigenen und migrantischen Landnutzer*innen, jedoch auch innerhalb dieser Gruppen.

Indigene Landnutzer*innen erlangen in beiden Fallregionen Zugang zu Land im Wesentlichen über ihre Zugehörigkeit zu der Gruppe, die sich als erste in der Gegend niedergelassen hat (first settlement) und aufgrund dessen traditionelle Landrechtsansprüche erhebt ${ }^{8}$. In der Gegend des ScanFarmProjekts beziehen sich Landnutzer*innen für die Einforderung von Landrechten zudem auf die erstmalige Bestellung des Lands (first cultivation) durch sie selbst oder ihre Vorfahren ${ }^{9}$. Der Rückbezug auf die erste Niederlassung sowie die erste Bestellung des Lands ermöglicht es Landnutzer*innen, Ansprüche auf permanente Zugangsrechte auf Land geltend zu machen. Eine geläufige Bezeichnung für diese permanenten Landrechte ist die des customary free-

$8 \quad$ Landnutzerin aus Nsonyameye, Interview am 25.4.2016; Research Officer des Regional House of Chiefs der Northern Region, Interview am 8.3.2017; paramount-chief und elders von Tijo, Gruppendiskussion am 8.5.2016.

9 Sub-chief von Nsonyameye, Interview am 20.4.2016; Landnutzerin aus Nsonyameye, Interview am 24.4.2016; Landnutzerin aus Nsonyameye, Interview am 24.4.2016; Landnutzerin aus Nsonyameye, Interview am 27.4.2016; Landnutzer aus Nsonyameye, Interview am 28.4.2016; Landnutzerer aus Nsonyameye und Mitglieder der Agogoman mma kuo, Interview am 16.3.2017; Landnutzer aus Nsonyameye und Vorsitzender der Agogoman mma kuo, Interview am 16.3.2017. 
hold, des traditionellen Grundbesitzes ${ }^{10}$. Rechteinhaber*innen, die über einen customary freehold verfügen, können eigenständig über die Nutzung des Lands entscheiden, zahlen an niemanden Abgaben, können es eigenständig verpachten und an ihre Nachkommen vererben. Nach traditionellem Recht bleibt ein customary freehold auch dann bestehen, wenn Rechteinhaber*innen das Land über einen langen Zeitraum brach liegen lassen und auch nicht betreten, um Feuerholz zu sammeln oder darauf befindliche Bäume zu ernten ${ }^{11}$. Diese Rechte bezeichne ich im weiteren Verlauf des Buches als permanente Rechte.

Landnutzer*innen in der Gegend des ScanFarm-Projekts, die sich auf die erste Niederlassung ihrer Vorfahren in der Gegend berufen, um ihre Rechte an Land geltend $\mathrm{zu}$ machen, beziehen sich in der Regel auf einen lange zurückliegenden Zeitpunkt. Sie berichten, ihre Vorfahren seien in eine bis dato unbesiedelte Gegend gekommen, um sich dort niederzulassen und eine Gemeinschaft zu gründen. Da sie die Ersten in der Gegend waren, seien sie Indigene. Traditionelle Autoritäten kommen ebenfalls aus einer der Familien, die sich als erste in einer Gegend niedergelassen haben ${ }^{12}$.

Weitaus häufiger als auf die erste Niederlassung verweisen Landnutzer*innen in der Gegend des ScanFarm-Projekts darauf, dass sie oder ihre unmittelbaren Vorfahren die Ersten gewesen seien, die das Land bestellt haben. Dies seien der Ursprung und die Grundlage ihrer Rechte an Land ${ }^{13}$. Diese Argumentationsweise setzt a priori voraus, dass sie indigene Landnutzer*innen sind. Denn nach traditionellem Recht können sich nur indigene Landnutzer*innen Land aneignen, indem sie es urbar machen und bestellen $^{14}$. In der Gegend von ScanFarm ist es in der Vergangenheit einigen Landnutzer*innen gelungen, über die erstmalige Bestellung des Lands im

10 Landnutzer aus Nsonyameye und Vorsitzender der Agogoman mma kuo, Interview am 16.3.2017.

$11 \quad$ Landnutzer aus Nsonyameye, Interview am 28.4.2016.

12 Landnutzerin aus Nsonyameye, Interview am 25.4.2016.

13 Sub-chief von Nsonyameye, Interview am 20.4.2016; Landnutzerin aus Nsonyameye, Interview am 24.4.2016; Landnutzerin aus Nsonyameye, Interview am 24.4.2016; Landnutzerin aus Nsonyameye, Interview am 27.4.2016; Landnutzer aus Nsonyameye, Interview am 28.4.2016; Landnutzerer aus Nsonyameye und Mitglieder der Agogoman mma kuo, Interview am 16.3.2017; Landnutzer aus Nsonyameye und Vorsitzender der Agogoman mma kuo, Interview am 16.3.2017.

14 Landnutzerin aus Nsonyameye, Interview am 25.4.2016; Landnutzer aus Nsonyameye, Interview am 28.4.2016. 
Laufe der Zeit Zugang zu Landflächen von bis zu 500 acres - etwas mehr als 200 Hektar - zu erlangen ${ }^{15}$. Diese Flächen wurden jedoch nicht dauerhaft bestellt. Um die Böden zu schonen, betreiben die meisten Landnutzer*innen Wanderfeldbau (shifting cultivation), bei dem sie die Äcker regelmäßig brach liegen lassen, damit sich der Boden regenerieren kann.

Im Gegensatz zur ersten Niederlassung bezieht sich der Verweis auf die erste Bestellung des Lands meistens auf eine jüngere Vergangenheit ${ }^{16}$. Landrechtsansprüche, die sich auf eine jüngere Vergangenheit beziehen, werden wenn es zu Konkurrenz um Flächen kommt - eher durch Akteure, die Zugang $\mathrm{zu}$ Land kontrollieren infrage gestellt, als solche mit einer langen Geschichte. Gemeinschaften indigener Landnutzer*innen unter dem Vorsitz eines subchiefs, die erst in der jüngeren Vergangenheit - vor ein bis zwei Generationen - begonnen hatten, Land in einer bestimmten Gegend zu nutzen, wurden immer wieder von in der Hierarchie höher stehenden traditionellen Autoritäten darauf hingewiesen, dass sich ihr Land trotz permanenter Nutzungsrechte unter deren Zuständigkeit befinde ${ }^{17}$. Dies ereignete sich insbesondere im Rahmen der Ernennung eines neuen sub-chiefs durch den paramount chief, der Umsetzung (kleinerer) kommerzieller landwirtschaftlicher Vorhaben oder der Freigabe von traditionell verwaltetem Land als Weideland durch den paramount chief ${ }^{18}$. In keinem der berichteten Fälle hatte dies jedoch im Alltag direkte Auswirkungen für die Landnutzer*innen, deren Zugang zu Land sich dadurch nicht veränderte. Dementsprechend fochten Landnutzer*innen und sub-chiefs solche Aussagen des paramount chiefs nicht an.

In der Gegend des BioFuel Africa-Projekts waren - insbesondere für Frauen - kollektive Rechte an Land ein wichtiger Zugangsmechanismus. Diese Rechte ermöglichten es Frauen, Bäume auf gemeinschaftlich genutzten Flächen zu ernten und die Baumfrüchte für den Eigenbedarf oder den Verkauf weiterzuverarbeiten. Darüber gelang es ihnen ein zusätzliches Einkommen für die Familie zu erwirtschaften ${ }^{19}$.

15 Landnutzer aus Nsonyameye und Vorsitzender der Agogoman mma kuo, Interview am 16.3.2017.

16 Landnutzerin aus Nsonyameye, Interview am 25.4.2016.

17 Landnutzerin aus Nsonyameye, Interview am 25.4.2016.

18 Landnutzerin aus Nsonyameye, Interview am 25.4.2016; Landnutzerer aus Nsonyameye und Mitglieder der Agogoman mma kuo, Interview am 16.3.2017.

19 Landnutzer*innen und settler-chief aus Kpachaa, Gruppendiskussion am 11.5.2016; Landnutzer und settler-chief aus Parachanayilli, Gruppendiskussion am 13.5.2016; Landnutzer*innen und sub-chief von Tuya, Gruppendiskussion am 14.5.2016. 
Ein weiterer rechtebasierter Mechanismus, über den indigene und migrantische Landnutzer*innen in den Fallregionen Zugang zu Land erlangen, sind individuelle Vereinbarungen mit Personen, die über permanente $\mathrm{Zu}$ gangsrechte an Land verfügen. Diese Personen können traditionelle Autoritäten oder indigene Landnutzer*innen sein. Individuelle Vereinbarungen sind - wenn nicht anders abgemacht - zeitlich unbegrenzt. Sie garantieren jedoch keinen dauerhaft verlässlichen Zugang $\mathrm{zu}$ Land, da sie jederzeit nach der Ernte aufgekündigt werden können. Meistens nehmen individuelle Vereinbarungen die Form mündlicher Absprachen an, nur selten halten die beiden Parteien sie schriftlich fest ${ }^{20}$. In beiden Fallregionen sind individuell vereinbarte Zugangsrechte an die Nutzung des Lands geknüpft. Aus der Bestellung des Lands leiten sich wiederum temporäre Rechtsansprüche ab. Diese gelten gleichermaßen für indigene und migrantische Landnutzer*innen. Solange Landnutzer*innen im Rahmen individueller Vereinbarungen Land bestellen, können sie nach traditionellem Recht Ansprüche darauf geltend machen. Im Falle des Verlusts dieses Lands haben sie zum Beispiel ein Anrecht auf die noch ausstehende Ernte oder - beim Verlust der Ernte - auf entsprechende Entschädigungszahlungen ${ }^{21}$. Die Rechte auf Land von Indigenen und Migrant*innen unterscheiden sich jedoch, wenn der Zugang zu Land über eine traditionelle Autorität ermöglicht wurde. Indigenen steht im Fall des Verlusts ihres Lands ein neues Stück Land zu, Migrant*innen haben darauf keinen Anspruch ${ }^{22}$.

Neben Landnutzungsvereinbarungen zwischen traditionellen Autoritäten und Landnutzer*innen ist sharecropping eine gängige Art der individuellen Vereinbarung ${ }^{23}$. Sharecropping-Vereinbarungen ermöglichen es Landnutzer*innen ohne bestehende Landrechtsansprüche, als Pächter*innen Nutzungsrechte zu erlangen. Diese Nutzungsrechte können sie über Inhaber*innen permanenter Landrechte erhalten. Sharecropping verstehe ich als rechtebasierten Zugangsmechanismus, da die beteiligten Akteure aufgrund zuvor bestehender oder neuverhandelter Rechte Zugang zu Land haben. Pächter*innen erlangen Zugang über die Nutzung des Lands, Rechteinha-

\footnotetext{
20 Landnutzer aus Nsonyameye, Interview am 25.4.2016.

21 Landnutzer aus Nsonyameye, Interview am 28.4.2016.

22 Landnutzer aus Nsonyameye, Interview am 25.4.2016.

23 Landnutzerin aus Nsonyameye, Interview am 24.4.2016; Landnutzerin aus Nsonyameye, Interview am 24.4.2016; Landnutzerin aus Nsonyameye, Interview am 27.4.2016.
} 
ber*innen halten ihren Zugang zu Land über die Abgaben der Pächter*innen aufrecht.

Neben sharecropping sind in der Gegend von ScanFarm auch klassische Pachtverträge eine verbreitete Form der individuellen Vereinbarung. Anders als beim sharecropping verlangen Pächter*innen für die Nutzung ihres Lands einen vorher festgesetzten Geldbetrag. Zum Zeitpunkt der Interviews, im April 2016, betrug dieser 200 GHS pro acre und Jahr, was damals umgerechnet knapp $30 €$ entsprach ${ }^{24}$.

$\mathrm{Zu}$ den individuellen Vereinbarungen zähle ich darüber hinaus die Formalisierung traditioneller Landrechtsansprüche. Diese Möglichkeit besteht für indigene Landnutzer*innen mit permanenten Landrechten, die sie schriftlich festhalten und durch traditionelle Autoritäten bestätigen lassen können. Als Formalisierung bezeichne ich die schriftliche Bestätigung mündlich ausgehandelter Rechte. Die Formalisierung traditioneller Landrechte erfolgt durch die schriftliche Bestätigung von Landrechten durch den für die Gegend zuständigen sub-chief sowie den jeweiligen paramount chief ${ }^{25}$. Eine solche Formalisierung ist eine Voraussetzung, wenn Landnutzer*innen traditionelle Landrechte bei der staatlichen Lands Commission registrieren lassen möchten. Sie dient jedoch insbesondere dazu, permanente Investitionen in Land wie bauliche Strukturen zur Weiterverarbeitung von landwirtschaftlichen Erzeugnissen - abzusichern. In der Gegend des BioFuel Africa-Projekts ist die Formalisierung traditioneller Landrechte nicht verbreitet. In derjenigen des ScanFarm-Projekts gibt es sie. Jedoch hat nur einer meiner Interviewpartner von der Formalisierung seiner traditionellen Landrechte Gebrauch gemacht. Mit 500 acres verfügte er über eine verhältnismäßig große Fläche, die er für die kommerzielle Landwirtschaft nutzte und auf der er eine Mühle zur Weiterverarbeitung von Maniok sowie einen Schweinezuchtbertrieb gebaut hat $^{26}$. Subsistenzwirtschaft betreibende Landnutzer*innen mit formalisierten Landrechten sind mir durch meine Forschung nicht bekannt.

24 Landnutzer*innen aus Nsonyameye, Baama \& Dukusen, Gruppendiskussion am 27.4.2016; Landnutzerin aus Nsonyameye, Interview am 25.4.2016; Landnutzer aus Nsonyameye, Interview am 25.4.2016.

25 Landnutzer aus Nsonyameye und Vorsitzender der Agogoman mma kuo, Interview am 16.3.2017.

26 Landnutzer aus Nsonyameye und Vorsitzender der Agogoman mma kuo, Interview am 16.3.2017. 


\section{Zugang zu Land über relationale und strukturelle Mechanismen}

Neben rechtebasierten Zugangsmechanismen sind in beiden Fallstudienregionen relationale und strukturelle Mechanismen für den Zugang zu Land relevant. Relationale und strukturelle Zugangsmechanismen ergeben sich aus den kontextspezifischen Bedingungen, unter denen Landnutzer*innen nach Zugang zu Land streben. Sie bezeichnen die Möglichkeiten, die sich aus diesen Bedingungen in Bezug auf den Zugang zu Land ableiten. Dazu zählen die Aushandlung sozialer und rechtlicher Ordnungen, in dessen Rahmen Akteure nach Zugang zu Land streben sowie Netzwerke und soziale Beziehungen, die Zugang zu Land ermöglichen können. Darüber hinaus beeinflussen Kategorien sozialer Zugehörigkeit wie Alter, Geschlecht, Klasse und Herkunft, die mit strukturell begründeten Ungleichheitsverhältnissen einhergehen, die Möglichkeiten, Rechte an Land einzufordern. Relationale und strukturelle Mechanismen sind eng mit rechtebasierten Zugangsmechanismen verschränkt. Relationale und strukturelle Mechanismen können - zum Beispiel vermittelt über soziale Zugehörigkeit - die Grundlage für die Einforderung von rechtebasiertem Zugang zu Land bilden oder seiner Absicherung dienen, wenn er infrage gestellt wird. Für Akteure, die selbst nicht auf rechtebasierte Zugangsmechanismen zurückgreifen können, erweitern relationale und strukturelle Mechanismen die Möglichkeiten, Zugang zu Land zu erlangen.

In beiden Fallregionen stützen relationale und strukturelle Mechanismen rechtebasierten Zugang zu Land. Landnutzer*innen handeln rechtebasierten Zugang zu Land innerhalb sozialer Beziehungen aus. Die Möglichkeiten der Aushandlung und der Einforderung traditioneller Landrechte ist eng an Kategorien sozialer Zugehörigkeit geknüpft. Zentral für die Aushandlung von rechtebasiertem Zugang zu Land ist in beiden Forschungsregionen die Kategorie Herkunft und damit verbundene Selbst- oder Fremdzuschreibungen der Landnutzer*innen (Indigene/Migrant*innen, Einheimische/Fremde, first comer/late comer). Argumente wie die erste Niederlassung in einer Gegend, die erste Bestellung des Lands oder die Aussage "meine Familie hat schon immer in dieser Gegend gelebt « veranschaulichen dies. Die Wirksamkeit solcher Argumentationsweisen impliziert, dass Indigenität eine gemeinschaftlich anerkannte Voraussetzung für die Einforderung permanenter Landrechte ist. Im Umkehrschluss leitet sich daraus ab, dass Landnutzer*innen aus 
anderen Gegenden nur eingeschränkten Zugang zu Land erhalten können ${ }^{27}$. Die Anerkennung als Indigene* $r$ durch Akteure, die Zugang zu Land kontrollieren, ist somit ein zentraler relationaler Zugangsmechanismus, der Rechte an Land nach sich ziehen kann. Im Umkehrschluss kann über die Kategorie Herkunft auch die Beschränkung von Rechten verdeutlicht werden. Indem Landnutzer*innen argumentieren, ihre Landrechte aufgrund der ersten Niederlassung ihrer Vorfahren in der Gegend oder der erstmaligen Bestellung des Lands erworben zu haben, fordern indirekt ihre Anerkennung als Indigene ein, um bestehende Rechte an Land gegenüber anderen Akteuren, die nach der Kontrolle über den Zugang zu Land streben, zu untermauern. Ebenso kann der Bezug auf Herkunft - zum Beispiel seitens traditioneller Autoritäten, die Zugang zu Land kontrollieren - dazu dienen, um jemandes Indigenität infrage zu stellen und Zugang zu Land zu beschränken ${ }^{28}$.

Neben Herkunft als Kategorie sozialer Zugehörigkeit sind in beiden Fällen auch Geschlecht und Alter als Analysekategorien relevant für den Zugang $\mathrm{zu}$ Land. Anders als Herkunft führen Landnutzer*innen Geschlecht und Alter jedoch nicht an, um Zugang zu Land zu erlangen oder zu konsolidieren. Vielmehr lassen sich entlang dieser Kategorien Einschränkungen des Zugangs zu Land oder Schwierigkeiten ihn einzufordern oder aufrechtzuerhalten nachvollziehen. Geschlecht ist in beiden Gegenden für die Analyse von Zugang zu Land relevant, da Frauen Zugang zu Land oftmals nur über männliche Familienmitglieder (Vater, Ehemann) erhalten ${ }^{29}$. In der Gegend des BioFuel AfricaProjekts spielen für die geschlechterspezifische Analyse von Zugang zu Land Tätigkeiten eine zentrale Rolle, die ausschließlich Frauen ausüben. $\mathrm{Zu}$ diesen Tätigkeiten zählen die Herstellung von Sheabutter und Dawadawa (einem fermentierten Gewürz aus den Früchten des Johannisbrotbaums) oder das Sammeln von Resten, die nach der maschinellen Ernte durch Landwirt*innen, die das Land zu kommerziellen Zwecken nutzen, auf dem Feld verbleiben ${ }^{30}$.

Alter ist aus folgenden Gründen eine relevante Kategorie für die Analyse des Zugangs zu Land. In der Gegend von ScanFarm berichteten einige Land-

27 Paramount-chief und elders von Tijo, Gruppendiskussion am 8.5.2016; Landnutzerin aus Nsonyameye, Interview am 24.4.2016.

28 Landnutzer*innen und settler-chief aus Kpachaa, Gruppendiskussion am 11.5.2016.

29 Landnutzerin aus Nsonyameye, Interview am 25.4.2016; Research Officer des Regional House of Chiefs der Northern Region, Interview am 8.3.2017; Mitarbeiterin der Civil Society Coalition on Land, Interview am 22.3.2016.

30 Landnutzer*innen und sub-chief von Tuya, Gruppendiskussion am 14.5.2016. 
nutzer*innen, dass sie aufgrund ihres Alters nicht mehr ausreichend darüber informiert seien, wie ihr Land aktuell genutzt würde. Denn sie selbst seien nicht mehr auf den Feldern tätig, sondern diese würden durch ihre Kinder oder durch Pächter*innen bestellt ${ }^{31}$. Zudem hätten sie nicht mehr die Kraft ihre Landrechte aktiv einzufordern, indem sie Unbefugte daran hinderten ihr Land zu nutzen ${ }^{32}$.

Schließlich spielen in beiden Fällen persönliche Beziehungen und Netzwerke eine wichtige Rolle für den Zugang zu Land. Über Beziehungen und Netzwerke kann bestehender Zugang zu Land gefestigt werden und sie erlauben denjenigen Landnutzer*innen Zugang zu Land zu erlangen, die aufgrund ihrer sozialen Zugehörigkeit nicht die Möglichkeit haben, traditionelle Landrechte geltend zu machen. Dies betrifft zum Beispiel junge Menschen - insbesondere Frauen - oder Migrant*innen. In beiden Gegenden haben zahlreiche Landnutzer*innen Zugang zu Land dank familiärer Beziehungen zu Rechteinhaber*innen: junge Menschen erlangen Zugang zu Land zunächst über ihre Eltern, Frauen nach der Heirat oftmals über ihren Mann ${ }^{33}$. Migrant*innen sind auf gute Beziehungen zu indigenen Landnutzer*innen angewiesen, um Zugang zu Land zu erlangen. Gute Beziehungen pflegen sie, indem sie sich in der und für die Gemeinschaft engagieren und sich als vertrauenswürdig erweisen. Dazu gehört, dass sie zu Festlichkeiten in der Gemeinschaft beitragen und ihren Respekt gegenüber traditionellen Autoritäten in Form sporadischer Ernteabgaben zeigen. Auf diese Weise integrieren sie sich in die Gemeinschaft, wodurch sie ihren Zugang zu Land festigen und über mehrere Generationen aufrechterhalten können ${ }^{34}$.

Beziehungen sind in beiden Gegenden wichtig für den Zugang zu Land und insbesondere für die Aufrechterhaltung von Landrechten. In der Gegend von ScanFarm betonen Landnutzer*innen und sub-chiefs die Bedeutung von Respekt und Treue gegenüber den traditionellen Autoritäten, die Zugang zu Land kontrollieren und denen sie ihren Zugang zu Land verdanken ${ }^{35}$. Für vie-

\footnotetext{
31 Landnutzerin aus Nsonyameye, Interview am 25.4.2016.

32 Landnutzerin aus Nsonyameye, Interview am 25.4.2016; Landnutzer aus Dukusen, Interview am 26.4.2016.

33 Mitarbeiterin der Civil Society Coalition on Land, Interview am 22.3.2016.

34 Landnutzer aus Nsonyameye, Interview am 25.4.2016; Landnutzer aus Changolinaaya, Interview am 12.5.2016; Landnutzer und settler-chief aus Parachanayilli, Gruppendiskussion am 13.5.2016.

35 Landnutzer aus Dukusen, Interview am 26.4.2016; sub-chief von Dukusen, Interview am 19.4.2016; sub-chief von Nsonyameye, Interview am 20.4.2016.
} 
le Landnutzer*innen ist das der sub-chief, für viele sub-chiefs der in der Hierarchie übergeordnete paramount chief. Ähnliches gilt auch für Landnutzer*innen in der Gegend des BioFuel Africa-Projekts. Dort ist vor allem für die settler communities die Beziehung zu dem sub-chief, der das Land verwaltet und es ihnen zur Verfügung gestellt hat, wichtig für ihren Zugang zu Land ${ }^{36}$. In der Gegend von ScanFarm sind Beziehungen des Weiteren zentral, um rechtebasierte Zugangsmechanismen wie sharecropping aushandeln zu können. In der Gegend des BioFuel Africa-Projekts trugen zudem persönliche Beziehungen zu Landwirt*innen aus Tamale, die Land für die kommerzielle Landwirtschaft gepachtet hatten, zur Ausweitung des eigenen Zugang zu Land zu bei. Diese Beziehungen ermöglichten es den Landnutzer*innen aus der Gegend Reste, die nach der maschinellen Ernte durch die Landwirt*innen auf den Feldern verblieben, manuell für den Eigenbedarf aufzusammeln ${ }^{37}$.

Netzwerke sind relevant für den Zugang zu Land, da über ihre Mitglieder Wissen und Informationen vermittelt werden, die Einfluss auf Zugang zu Land haben können. Mithilfe von Kenntnissen traditioneller Landrechte gelingt es Landnutzer*innen Argumente zu entwickeln, um Rechte an Land einfordern zu können. Auch für die Anerkennung von Rechten an Land sind Netzwerke relevant, indem ihre Mitglieder beispielweise den Verlauf von Grenzen zwischen den Feldern einzelner Landnutzer*innen oder unterschiedlicher Gemeinschaften bestätigen ${ }^{38}$.

Persönliche und professionelle Netzwerke sowie Netzwerke, die an soziale Positionen geknüpft sind, können ebenfalls Zugang zu Land festigen, indem sie sich vorteilhaft auf andere Zugangsmechanismen auswirken. Dies erwies sich insbesondere im Fall von ScanFarm als relevant. So ermöglichte dort die Mitgliedschaft im ATC oder die gute Beziehung zu Mitgliedern des ATC teilweise privilegierten Zugang zu Informationen bezüglich des Lands sowie finanzielle Zuwendungen, die über die Vergabe von Land generiert werden $^{39}$. Auch professionelle Netzwerke wirkten sich auf den Zugang zu Land

36 Landnutzer aus Changolinaaya, Interview am 12.5.2016; Landnutzer und settler-chief aus Parachanayilli, Gruppendiskussion am 13.5.2016.

37 Landnutzer*innen und sub-chief von Tuya, Gruppendiskussion am 14.5.2016.

38 Landnutzerer aus Nsonyameye und Mitglieder der Agogoman mma kuo, Interview am 16.3.2017; Landnutzer aus Nsonyameye und Vorsitzender der Agogoman mma kuo, Interview am 16.3.2017.

Sub-chief von Dukusen, Interview am 19.4.2016; sub-chief von Nsonyameye, Interview am 20.4.2016; Registerführer des Agogo Traditional Council und Leiter des Agogo Customary Land Secretariat, Interview am 20.4.2016. 
aus. Ein Beispiel dafür sind die Agogoman mma kuo (weltweite Bürger*innen von Agogo), ein Netzwerk, in dem sich Bürger*innen aus Agogo zusammengeschlossen haben, von denen viele lange Zeit im Ausland gelebt haben oder immer noch leben. Ihr Anliegen besteht darin, sich für die Belange der Bewohner*innen von Agogo einzusetzen und die »Entwicklung« Agogos mithilfe internationaler Unterstützung voranzutreiben. Dazu zählt auch die Stärkung von Rechten an Land. Viele Mitglieder dieses Netzwerks haben ein hohes Bildungsniveau, weshalb ein Engagement in diesem Netzwerk den Austausch von Wissen - zum Beispiel in Bezug auf Landrechte - begünstigt und eine gemeinsame Einforderung dieser Rechte - zum Beispiel über das staatliche Rechtssystem - erleichtert ${ }^{40}$. So konnten Netzwerke und soziale Beziehungen Einschränkungen des Zugangs zu Land aufgrund strukturell bedingter Ungleichheitsverhältnisse zum Teil abmildern.

\subsection{Land grabbing und die Veränderung von Kontrolle und Zugang}

Mit der Vergabe des Lands an ScanFarm und BioFuel Africa haben sich die Kontrolle über den Zugang zu Land und die Möglichkeiten Zugang zu Land zu erlangen oder aufrechtzuhalten in beiden Gegenden maßgeblich gewandelt. In erster Linie veränderte sich in diesem Kontext in beiden Fällen die Kontrolle über den Zugang zu Land. Darüber hinaus wurden einige Mechanismen weniger relevant, um Zugang zu Land (aufrecht)erhalten zu können, andere hingegen gewannen im Rahmen der Vergabe des Lands an die Unternehmen an Bedeutung. Wie zu Beginn des Kapitels dargestellt, übten vor der Vergabe des Lands an ScanFarm und BioFuel Africa verschiedene Akteure Kontrolle über den Zugang zu Land aus: der paramount chief, die sub-chiefs sowie Landnutzer*innen mit permanenten Landrechten. Sie konnten in unterschiedlichen Kontexten bestimmen, wer einen Nutzen aus Land ziehen kann. Mit der Vergabe des Lands an die Unternehmen änderte sich dies.

In beiden Fällen entschieden die paramount chiefs über die Vergabe des Lands, ohne sich mit den betroffenen Landnutzer*innen und sub-chiefs zu beraten. Über die Vergabe des Lands an die Unternehmen verloren die sub-chiefs

40 Landnutzerer aus Nsonyameye und Mitglieder der Agogoman mma kuo, Interview am 16.3.2017; Landnutzer aus Nsonyameye und Vorsitzender der Agogoman mma kuo, Interview am 16.3.2017. 
und Landnutzer*innen in beiden Gegenden - wenn auch in unterschiedlichem Ausmaß - die Kontrolle über den Zugang zu ihrem Land. Die paramount chiefs ließen die sub-chiefs und Landnutzer*innen nicht an den Verhandlungen um die Vergabe des Lands teilhaben und gaben ihnen nicht die Möglichkeit, ihre Meinung zu dem Vorhaben zum Ausdruck zu bringen ${ }^{41}$. Damit ignorierten die paramount chiefs indirekt bestehende Rechte und individuelle Vereinbarungen. Im Fall von ScanFarm wusste zu Beginn nur der sub-chief von Dukusen von der Landvergabe, da sein Neffe den Kontakt zu den Investoren hergestellt hatte ${ }^{42}$. Die anderen sub-chiefs sowie die Landnutzer*innen erhielten zunächst keine Informationen. Sie wurden später, als das Unternehmen bereits mit der Nutzung des Lands begonnen hatte, vor vollendete Tatsachen gestellt ${ }^{43}$. Die Landvergabe an BioFuel Africa unterschied sich im Vergleich dazu. Anders als im Fall von ScanFarm setzte sich der Geschäftsführer von BioFuel Africa dafür ein, dass alle Landnutzer*innen aus der Gegend im Rahmen einer öffentlichen Veranstaltung vor Beginn der Landnutzung über das Vorhaben informiert wurden ${ }^{44}$. Diese Maßnahme bedeutete jedoch nicht, dass sub-chiefs und Landnutzer*innen die Möglichkeit bekamen, an den Verhandlungen um die Landvergabe teilzunehmen ${ }^{45}$. Mithilfe von Informant*innen versuchte das Unternehmen allerdings zu erheben, wo sich die Felder der Landnutzer*innen befanden, um diese wenn möglich von der Umnutzung des Lands zu verschonen (Tsikata/Yaro 2011). Welche Landnutzer*innen das

41 Landnutzer*innen aus Nsonyameye, Baama \& Dukusen, Gruppendiskussion am 27.4.2016; Landnutzerin aus Nsonyameye, Interview am 24.4.2016; Landnutzerin aus Nsonyameye, Interview am 24.4.2016; Landnutzer aus Nsonyameye, Interview am 25.4.2016; Landnutzerer aus Nsonyameye und Mitglieder der Agogoman mma kuo, Interview am 16.3.2017; Landnutzerin aus Dukusen, Interview am 16.3.2017; Abteilungsleiterin der Regional Lands Commission der Ashanti Region, Interview am 13.04.2016; Leiter des Department of Agriculture des Asante Akyem North District, Interview am 22.4.2016, Projektleiter des Land and Resource Management Center, Interview am 12.4.2016.

42 Sub-chief von Dukusen, Interview am 19.4.2016.

43 Landnutzerin aus Nsonyameye, Interview am 24.4.2016; Landnutzer aus Nsonyameye, Interview am 25.4.2016; Landnutzerin aus Nsonyameye, Interview am 27.4.2016; Projektleiter des Land and Resource Management Center, Interview am 12.4.2016.

44 Landnutzer*innen und settler-chief aus Kpachaa, Gruppendiskussion am 11.5.2016.

45 Landnutzer*innen und settler-chief aus Kpachaa, Gruppendiskussion am 11.5.2016; Landnutzer*innen und sub-chief von Tuya, Gruppendiskussion am 14.5.2016; Landnutzer aus Changolinaaya, Interview am 12.5.2016. 
Unternehmen oder der paramount chief tatsächlich über das Vorhaben informierten und wessen Felder identifiziert wurden, war mit den Mechanismen verbunden, über die Landnutzer*innen bislang Zugang zu Land erlangten. Migrantische Landnutzer*innen, an die der paramount chief selbst Land vergeben hatte sowie diejenigen aus seiner Gemeinschaft informierte er zeitnah selbst. Indigene sub-chiefs und Landnutzer*innen erhielten die Informationen teilweise über das Unternehmen und teilweise über den paramount chief. Settler communities sowie individuelle Pächter"innen, die ihr Land über einen sub-chief erhalten hatten, waren diejenigen mit dem unsichersten Zugang zu Land. Sie informierte weder der paramount chief noch das Unternehmen.

Im Folgenden analysiere ich entlang der rechtebasierten, relationalen und strukturellen Zugangsmechanismen, inwiefern die Verschiebung der Kontrolle des Zugangs mit einer Veränderung des Zugangs zu Land einhergeht.

\section{Veränderung des Zugangs zu Land über rechtebasierte Mechanismen}

Vor der Vergabe des Lands an ScanFarm und BioFuel Africa waren rechtebasierte Mechanismen in Form traditioneller Landrechte und individueller Vereinbarungen sowie relationale und strukturelle Mechanismen, wie soziale Zugehörigkeit, soziale Beziehungen und Netzwerke die zentralen Mittel, über die Landnutzer*innen Zugang zu Land erlangten. Mit der Verschiebung der Kontrolle des Zugangs weg von den sub-chiefs und Landnutzer*innen hin zu den paramount chiefs verringerten sich die Möglichkeiten der Landnutzer*innen über zuvor relevante Zugangsmechanismen ihren Zugang zu Land aufrechtzuerhalten. In den Verhandlungen um die Vergabe des Lands zwischen den Unternehmen und den paramount chiefs fanden traditionelle Landrechte $\mathrm{zu}$ nächst keine Berücksichtigung, was für die meisten Landnutzer*innen zum Verlust oder zu einer Verschlechterung des Zugangs zu Land führte.

Im Fall von ScanFarm konnten Landnutzer*innen mit permanenten traditionellen Landrechten die Nutzung des Lands nicht mehr selbst kontrollieren. Mit Erlaubnis des paramount chiefs begann das Unternehmen das Land in Abwesenheit der Landnutzer*innen mit Traktoren zu bearbeiten. Auch nach Aufforderung durch die Rechteinhaber*innen, das Land zu verlassen, verfolgte das Unternehmen sein Vorhaben weiter ${ }^{46}$. Arbeiter*innen des Unter- 
nehmens rodeten Bäume in der Nacht, um Widerstand der Landnutzer*innen zu vermeiden ${ }^{47}$. Durch die Arbeiten auf dem Land entfernte das Unternehmen natürliche Grenzmarkierungen, die es Rechteinhaber*innen zuvor ermöglicht hatten, Lage und Größe ihres Landes zu bestimmen ${ }^{48}$. Die einzige Möglichkeit weiterhin einen Nutzen aus ihrem Land ziehen zu können, war die Einforderung von Entschädigungszahlungen. Entschädigungszahlungen konnten Landnutzer*innen, die zuvor über permanente Rechte auf Land verfügten - also Zugang zu Land kontrollierten - einfordern. Sie erhielten diese jedoch nicht automatisch. Das Unternehmen kam nicht direkt auf die Landnutzer*innen zu, um sie für den Verlust ihres Lands zu entschädigen. Um Entschädigungszahlungen in Anspruch nehmen zu können, mussten Landnutzer*innen zunächst ihre zuvor bestehenden traditionellen Rechte beweisen und in diesem Zuge Einschränkungen hinnehmen. Zum Zeitpunkt der Verhandlung über Entschädigungszahlungen hatte das Unternehmen Grenzmarkierungen bereits entfernt oder zerstört. Aufgrund dieser fehlenden Grenzmarkierungen war es für viele Landnutzer*innen schwierig, die Lage ihres Landes und damit einhergehend dessen Größe zu bestimmen. In der Berechnung der Entschädigungszahlungen berücksichtigte das Unternehmen daher oftmals nur Flächen die kleiner waren, als diejenigen, an denen Landnutzer*innen zuvor traditionelle Landrechte hatten ${ }^{49}$. Das Unternehmen zahlte Landnutzer*innen einen einmaligen Betrag von 30 GHS pro acre sowie eine jährliche Entschädigungssumme von 10 GHS pro acre. Gemessen an dem zuvor über das Land generierte Einkommen waren die Entschädigungszahlungen darüber hinaus für viele Landnutzer*innen nachteilig $^{50}$. Diejenigen, die ihr Land vor der Vergabe an das Unternehmen ver-

47 Landnutzerin aus Nsonyameye, Interview am 27.4.2016; Landnutzerin aus Dukusen, Interview am 16.3.2017; Landnutzer aus Nsonyameye und Vorsitzender der Agogoman mma kuo, Interview am 16.3.2017.

48 Landnutzer"innen aus Nsonyameye, Baama \& Dukusen, Gruppendiskussion am 27.4.2016; Landnutzerin aus Nsonyameye, Interview am 24.4.2016; Landnutzerin aus Nsonyameye, Interview am 26.4.2016; Landnutzerin aus Nsonyameye, Interview am 27.4.2016; Landnutzer aus Nsonyameye und Vorsitzender der Agogoman mma kuo, Interview am 16.3.2017.

49 Landnutzerin aus Nsonyameye, Interview am 26.4.2016; Landnutzer aus Nsonyameye, Interview am 28.4.2016; Landnutzerer aus Nsonyameye und Mitglieder der Agogoman mma kuo, Interview am 16.3.2017; Landnutzer aus Nsonyameye und Vorsitzender der Agogoman mma kuo, Interview am 16.3.2017.

50 Landnutzerin aus Nsonyameye, Interview am 24.4.2016; Landnutzerin aus Nsonyameye, Interview am 24.4.2016; Landnutzerin aus Nsonyameye, Interview am 25.4.2016; 
pachtet hatten, erhielten 200 GHS Pacht pro acre und Jahr ${ }^{51}$. Im Rahmen von sharecropping-Vereinbarungen bekamen sie einen Teil der Ernte, der vom Wert vergleichbar mit Pachteinnahmen war, wie einen Sack Mais pro acre ${ }^{52}$. Die Entschädigungszahlungen durch das Unternehmen waren somit 20-mal geringer. Trotz bestehender Rechte, die das Unternehmen durch die Leistung von Entschädigungszahlungen anerkannte, verschlechterte sich der Zugang zu Land im Kontext der Landvergabe. Der finanzielle Nutzen, den Landnutzer*innen über die Entschädigungszahlungen aus dem Land zogen, war deutlich geringer ist als derjenige, den sie zuvor durch das Land hatten.

Für Landnutzer*innen, deren Rechte an die Nutzung des Lands gebunden waren - zum Beispiel im Rahmen individueller Vereinbarungen - hatte die Veränderung der Zugangskontrolle zur Folge, dass sie ihren Zugang $\mathrm{zu}$ Land vollständig verloren. Im Gegensatz zu Landnutzer*innen mit permanenten Rechten waren ihre Ansprüche auf Entschädigungszahlungen sehr gering: das Unternehmen entschädigte diese Landnutzer*innen nur für den Verlust ihrer Ernte. Wenn sie das Land in dem Zeitraum, in dem es vergeben wurde, jedoch nicht bestellt hatten - weil zum Beispiel keine Anbausaison war - oder sie nicht beweisen konnten, dass sie ihre Ernte durch die Arbeiten des Unternehmens verloren hatten, erhielten sie keine Entschädigung ${ }^{53}$.

Vorteilhaft waren die Entschädigungszahlungen nur für ältere Landnutzer*innen, die ihr Land vor der Vergabe an ScanFarm nicht mehr selbst genutzt oder verpachtet, sondern es brach liegen lassen hatten. Für sie bedeuteten die Entschädigungszahlungen ein, wenn auch geringes, regelmäßiges Einkommen und damit eine Verbesserung des Zugangs zu Land ${ }^{54}$. Land, das lange Zeit brach liegt, ist jedoch selten ungenutzt. Mitglieder der Gemeinschaft nutzen es für vielfältige Zwecke.

Eine weitere Veränderung rechtebasierter Zugangsmechanismen, die mit der Verschiebung der Zugangskontrolle einherging, ist die neue Bedeutung, die formalisierte traditionelle Landrechte für Zugang zu Land bekommen.

Landnutzer aus Nsonyameye, Interview am 25.4.2016; Landnutzerin aus Nsonyameye, Interview am 26.4.2016; Landnutzerin aus Nsonyameye, Interview am 27.4.2016; Landnutzerin aus Nsonyameye, Interview am 27.4.2016.

51 Landnutzer aus Nsonyameye, Interview am 25.4.2016; Landnutzerin aus Nsonyameye, Interview am 27.4.2016; Landnutzerin aus Nsonyameye, Interview am 27.4.2016; Landnutzer*innen aus Nsonyameye, Baama \& Dukusen, Cruppendiskussion am 27.4.2016.

52 Landnutzer aus Nsonyameye, Interview am 25.4.2016.

53 Landnutzer aus Nsonyameye, Interview am 25.4.2016.

54 Sub-chief von Nsonyameye, Interview am 20.4.2016. 
Vor der Vergabe des Lands an ScanFarm hatte die Formalisierung traditioneller Landrechte keinen direkten Nutzen für den Zugang zu Land. Um die Kontrolle über den Zugang zu Land nach der Vergabe des Lands wieder zurückgewinnen $\mathrm{zu}$ können, erwiesen sich formalisierte traditionelle Landrechte jedoch als unabdingbar. Im Vergleich $\mathrm{zu}$ nicht-formalisierten traditionellen Rechten sind sie leichter zu beweisen. Dennoch verloren auch Landnutzer*innen mit formalisierten Rechten im Rahmen der Landvergabe die Kontrolle über den Zugang zu ihrem Land. Da das Land - wenn auch ohne das Wissen der Rechteinhaber*innen - Teil des Pachtvertrags war, erhob das Unternehmen Anspruch darauf ${ }^{55}$. Zwar bestätigte der paramount chief gegenüber den Landnutzer*innen ihre formalisierten Rechte, setzte sie jedoch nicht gegenüber dem Unternehmen durch. Somit bewirkte die Formalisierung keine automatische Anerkennung der Rechte durch das Unternehmen. Die einzige Möglichkeit die Rechteinhaber* innen blieb, um ihre Landrechte einzufordern, war der staatliche Rechtsweg, also eine Klage gegen die Nutzung des Lands durch das Unternehmen ${ }^{56}$. Landnutzer*innen ohne formalisierte Landrechte, also ohne Landrechte die durch traditionelle Autoritäten schriftlich bestätigt waren, hatten diese Möglichkeit nicht. Das Gerichtsverfahren am Kumasi High Court begann im Januar 2014 und war zur Zeit des letzten Interviews im März 2017 noch nicht entschieden ${ }^{57}$. Formalisierte Landrechte ermöglichten Landnutzer*innen somit zwar für ihre Rechte zu kämpfen, sicherten jedoch keinen direkten Zugang zu Land.

Durch die Einforderung von Landrechten über das staatliche Rechtssystem gewannen staatliche Akteure an Einfluss in der Durchsetzung traditioneller Rechte. Zuvor hatten diese keinerlei Einfluss auf traditionelle Landrechte. Im Kontext der Landvergabe ist die Einbeziehung staatlicher Akteure (Richter*innen, Staatsanwält"innen) jedoch unabdingbar, um zuvor bestehenden Zugang zu Land über traditionelle Rechte durchsetzen zu können. Um das Unternehmen erfolgreich daran hindern zu können, Land - das nach

55 Landnutzerer aus Nsonyameye und Mitglieder der Agogoman mma kuo, Interview am 16.3.2017; Landnutzer aus Nsonyameye und Vorsitzender der Agogoman mma kuo, Interview am 16.3.2017.

56 Landnutzerer aus Nsonyameye und Mitglieder der Agogoman mma kuo, Interview am 16.3.2017; Landnutzer aus Nsonyameye und Vorsitzender der Agogoman mma kuo, Interview am 16.3.2017.

57 Landnutzer aus Nsonyameye und Vorsitzender der Agogoman mma kuo, Interview am 16.3.2017. 
Meinung des Unternehmens offiziell Teil des Pachtvertrags war - mit schweren Maschinen zu bearbeiten, benötigen Landnutzer"innen einen Beweis in Form eines staatlich bestätigten Dokuments, das ihre Rechte an diesem Land belegte

Die Landvergabe an BioFuel Africa unterschied sich von derjenigen an ScanFarm. Gleich ist jedoch, dass sich auch in diesem Fall die Kontrolle des $\mathrm{Zu}$ gangs zu Land weg von sub-chiefs und Landnutzer*innen hin zum paramount chief verschob. Anders als im Fall von ScanFarm setzte sich das Unternehmen nach Rücksprache mit dem paramount chief und staatlichen Akteuren wie Mitarbeiter* innen der Environmental Protection Agency (EPA) und der Regional Lands Commission dafür ein, dass Landnutzer*innen mit traditionellen Rechten an dem Land über das Vorhaben informiert und ihre Landrechte berücksichtigt werden (Bruce 2008; SGS Environment 2009; Tsikata/Yaro 2011). Im Rahmen einer Informationsveranstaltung in Kumasi, zu der chiefs und Landnutzer* innen eingeladen waren, informierte das Unternehmen über das geplante Vorhaben und gab den Anwesenden die Möglichkeit, Fragen diesbezüglich zu stellen. Darüber hinaus strebte es an, die genutzten Felder zu identifizieren. Wenn möglich wollte es diese von ihrem Vorhaben verschonen oder ansonsten eine Alternative zur Verfügung zu stellen, indem es bislang unbestelltes Land außerhalb der gepachteten Fläche für die Landnutzer*innen rodete und pflügte (Bruce 2008; Tsikata/Yaro 2011). Die Unternehmenspolitik ermöglichte es indigenen Landnutzer*innen mit permanenten Landrechten im Rahmen der Vergabe des Lands an BioFuel Africa ihren eigenen Zugang zu Land aufrechtzuerhalten. Das an das Unternehmen vergebene Land ist relativ dünn besiedelt und die meisten Dörfer sind sehr klein. Das ermöglichte dem Unternehmen die Felder für den Jatropha-Anbau so anzulegen, dass es die Dörfer sowie die um die Dörfer liegenden Felder der Landnutzer*innen aussparte und dennoch eine zusammenhängende Fläche bewirtschaften konnte (SGS Environment 2009).

Eine maßgebliche Beschränkung des Zugangs zu Land zog die Verschiebung der Zugangskontrolle jedoch für Nutzer*innen nach sich, die nicht über permanente, individuelle Rechte an Land verfügen, sondern Zugang $\mathrm{zu}$ gemeinschaftlich genutztem Land über kollektive Rechte hatten. Dieses Land zeichnete sich dadurch aus, dass eine Vielzahl an wirtschaftlich nutzbaren Bäumen darauf wuchs. In Vorbereitung der gepachteten Fläche für den Jatropha-Anbau rodete das Unternehmen Teile dieses gemeinschaftlich genutzten Lands um die Dörfer Kpachaa, Tuya, Jashei und Parachanayil- 
$\mathrm{li}^{58}$. Dies schränkte Zugang zu Land insbesondere für Frauen ein, da diese ihr Haupteinkommen durch die Verarbeitung von Baumfrüchten und den Verkauf der Endprodukte erwirtschafteten. Durch die Rodung der Bäume verloren sie diese Einkommensquelle. Während Landnutzer"innen mit permanenten Landrechten im Verlustfall ein alternatives Stück Land erhielten, räumte das Unternehmen Nutzer*innen mit kollektiven Rechten keinen Anspruch auf eine derartige Entschädigung ein. Gleiches galt für Migrant*innen, settler communities sowie Pächter*innen, die das Land für die kommerzielle Landwirtschaft nutzten, jedoch nicht vor Ort lebten. Sie alle hatten die Möglichkeit, sich eigenständig um ein neues Stück Land $\mathrm{zu}$ bemühen, konnten nach traditionellem Recht jedoch keinen Anspruch darauf erheben ${ }^{59}$. Da ihnen die Landnutzungsrechte jederzeit entzogen werden konnten, erhielten sie - solange sie ihre Ernte durch die Vergabe des Lands nicht verloren - auch keine Entschädigungszahlungen seitens des Unternehmens.

\section{Veränderung des Zugangs zu Land über relationale und strukturelle Mechanismen}

Vor der Vergabe des Lands an ScanFarm und BioFuel Africa ermöglichten oder festigten unterschiedliche Formen der Aushandlung von Landnutzungsrechten innerhalb sozialer Beziehungen Zugang zu Land. Landnutzer*innen erlangten Zugang zu Land durch Rückbezug auf Kategorien sozialer Zugehörigkeit, persönliche Beziehungen zu Rechteinhaber*innen oder das Engagement in professionellen Netzwerken. Im Kontext der Vergabe des Lands an ScanFarm und BioFuel Africa spielen relationale Mechanismen weiterhin eine Rolle für den Zugang zu Land. Durch die Verschiebung der Kontrolle des Zugangs verloren viele Mechanismen an Bedeutung. Nach der Vergabe des Lands an die Unternehmen waren die für Zugang zu Land bedeutsamen relationalen Zugangsmechanismen somit weniger divers als

58 Landnutzer*innen und settler-chief aus Kpachaa, Gruppendiskussion am 11.5.2016; Landnutzer und settler-chief aus Parachanayilli, Gruppendiskussion am 13.5.2016; Landnutzer*innen und sub-chief von Tuya, Gruppendiskussion am 14.5.2016; Landnutzer*innen und sub-chief von Jashei, Gruppendiskussion am 15.5.2016.

59 Landnutzer*innen und settler-chief aus Kpachaa, Gruppendiskussion am 11.5.2016; Landnutzer und settler-chief aus Parachanayilli, Gruppendiskussion am 13.5.2016; Research Officer des Regional House of Chiefs der Northern Region, Interview am 8.3.2017; I-StA-12. 
vorher. Sie beschränkten sich im Kern auf Beziehungen zu traditionellen Autoritäten, Beziehungen zu den Unternehmen sowie die erfolgreiche Anerkennung als indigene Landnutzer*innen durch Akteure, die Zugang zu Land kontrollieren, wie die paramount chiefs oder Unternehmen.

Die Einschränkung der rechtebasierten Zugangsmechanismen, die mit der Verschiebung der Kontrolle von Zugang zu Land einherging, wirkte sich ebenfalls auf relationale Zugangsmechanismen aus. Da sub-chiefs und Landnutzer*innen mit permanenten Landrechten die Kontrolle über den Zugang zu Land verloren, konnten persönliche Beziehungen $\mathrm{zu}$ ihnen und daraus hervorgehende mündliche Vereinbarungen keinen Zugang zu Land mehr gewährleisten. Nur Vereinbarungen, die mit dem paramount chief getroffen wurden, blieben weiterhin von Relevanz. Im Gegenzug dazu gewann soziale Zugehörigkeit an Bedeutung. Migrantische Landnutzer*innen sowie Pächter*innen aus anderen Gegenden waren die Verlierer*innen der Landvergabe. Sie haben keinen Anspruch darauf, für den Verlust von Zugang zu Land finanziell oder über die Bereitstellung von Alternativflächen entschädigt zu werden. Aber auch andere relationale und strukturelle Mechanismen gewannen an Relevanz für den Zugang zu Land. Wissen und Bildung gewannen beispielsweise im Kontext der Landvergabe an Bedeutung. Insbesondere der Fall der Landvergabe an ScanFarm lässt darauf schließen, dass Informationen zur Vergabe des Lands, Kenntnisse der eigenen Rechte und Bildung allgemein grundlegend waren, um Zugang zu Land aufrechtzuerhalten oder zurückzufordern. Bildung spielte eine zentrale Rolle für die Einforderung von Landrechten per Gerichtsverfahren, um Briefe an das Unternehmen, den paramount chief und Behörden schreiben und auf Landrechte aufmerksam machen zu können, um schriftliche Informationen zum Projekt von Behörden- oder Unternehmensseite lesen zu können und den Inhalt des Memorandum of Understanding bezüglich der Entschädigungszahlungen an die Landnutzer*innen durch das Unternehmen verstehen $\mathrm{zu}$ können ${ }^{60}$. Einige Interviews verdeutlichten, dass insbesondere ältere Personen mit einem schwachen Bildungshintergrund den Inhalt und die Reichweite des

60 Landnutzerer aus Nsonyameye und Mitglieder der Agogoman mma kuo, Interview am 16.3.2017; Landnutzer aus Nsonyameye und Vorsitzender der Agogoman mma kuo, Interview am 16.3.2017; Brief des Registerführers des ATC an den Geschäftsführer von ScanFarm, 4.1.2010; Brief der Landnutzerinnen von Baama an den Vorsitzenden der Asante Akyem North Distric Assembly, 1.2.2011; Brief der Landnutzerinnen von Baama an den Vorsitzenden der Asante Akyem North Distric Assembly, 16.2.2011. 
Memorandum of Understanding nicht gänzlich verstanden ${ }^{61}$. Ihnen war nicht klar, dass das Unternehmen eine einmalige Entschädigungszahlung pro acre Land leistet, die höher ist als die jährliche Zahlung. Sie gingen davon aus, dass sie diese einmalige Zahlung von 30 GHS pro acre von nun an jährlich erhalten würden, wobei die jährliche Entschädigungszahlung nur ein Drittel davon betrug. Darüber hinaus konnten sie nicht nachvollziehen, wie sich die Entschädigungszahlungen zusammensetzten und wie das Unternehmen die Größe ihres Lands berechnet hatte ${ }^{62}$. Im Kontext der Landvergabe sind ungleiche Möglichkeiten Zugang zu Land aufrechtzuhalten oder zurückzufordern somit stärker als vorher an soziale Zugehörigkeit und damit einhergehende soziale Differenzierung und strukturelle Ungleichheitsverhältnisse geknüpft.

Aufgrund der Aneignung der Kontrolle über Land durch die paramount chiefs, wurde im Kontext der Landvergabe die Beziehung zu ihnen zum zentralen Zugangsmechanismus. Sie wirkt sich auf den Erhalt und den Umfang von Informationen in Bezug auf die Vergabe des Lands aus und ermöglicht eine finanzielle Beteiligung an den Einnahmen aus der Landvergabe. Darüber hinaus führte eine enge Beziehung zum paramount chief zu privilegiertem $\mathrm{Zu}$ gang zu Arbeit für das Unternehmen oder Serviceleistungen, wie das maschinelle Roden oder Pflügen neuer Flächen durch das Unternehmen ${ }^{63}$ (Bruce 2009).

Im Fall von ScanFarm ermöglichte eine gute Beziehung zum paramount chief zeitnahe Informationen zu dem agrarindustriellen Vorhaben des Unternehmens ${ }^{64}$. So erfuhr zunächst der sub-chief von Dukusen von dem Vorhaben, da sein Neffe, die Beziehung zwischen dem paramount chief und den Investoren hergestellt hatte ${ }^{65}$. Darüber hinaus war die Beziehung zum paramount chief zentral dafür, wen er mit der Aufgabe betraute, dem Unterneh-

61 Landnutzerin aus Nsonyameye, Interview am 24.4.2016; Landnutzerin aus Nsonyameye, Interview am 26.4.2016; Landnutzerin aus Nsonyameye, Interview am 27.4.2016.

62 Landnutzerin aus Nsonyameye, Interview am 26.4.2016; Landnutzerin aus Nsonyameye, Interview am 27.4.2016.

63 Sub-chief von Baama, Interview am 20.04.2016, Registerführer des Agogo Traditional Council und Leiter des Agogo Customary Land Secretariat, Interview am 20.4.2016.

64 Sub-chief von Dukusen, Interview am 19.4.2016; sub-chief von Nsonyameye, Interview am 20.4.2016; sub-chief von Baama, Interview am 20.04.2016.

65 Landnutzer aus Dukusen, Interview am 26.4.2016; sub-chief von Dukusen, Interview am 19.4.2016; sub-chief von Baama, Interview am 20.04.2016; Registerführer des Agogo Traditional Council und Leiter des Agogo Customary Land Secretariat, Interview am 20.4.2016. 
men das zur Verfügung stehende Land zu zeigen. Aufgrund ihres Vertrauensverhältnisses erhielt der ehemalige sub-chief von Dukusen diese Aufgabe und nicht, wie eigentlich üblich, der amtierenden sub-chief ${ }^{66}$. Des Weiteren wirkte sich die Beziehung zu Mitgliedern des ATC positiv auf den Zugang zu Arbeit für ScanFarm aus. Während ScanFarm nur wenige Landnutzer*innen aus den Dörfern als Hilfsarbeiter*innen auf saisonaler Basis einstellte, erhielt der Sohn des Registrators (registrar) des ATC eine permanente Einstellung als Fahrer für das Unternehmen, die Tochter des paramount chiefs arbeitete im Management des Unternehmens und der ehemalige sub-chief von Dukusen erhielt eine Festanstellung als Arbeiter ${ }^{67}$. Andere Zugangsmechanismen wie Beziehungen zu individuellen Rechteinhaber*innen und daraus hervorgehende Vereinbarungen zur Nutzung von Land konnten nach der Vergabe des Lands an das Unternehmen keinen Zugang zu Land mehr gewährleisten.

Auch im Fall von BioFuel Africa spielte im Kontext der Landvergabe die Beziehung zum Tijo Naa, dem dortigen paramount chief eine entscheidende Rolle für den Zugang zu Land der Landnutzer*innen. Sie hatte Einfluss darauf, ob und zu welchem Zeitpunkt der Tijo Naa die landnutzenden Gemeinschaften über die Umnutzung des Lands informierte oder ob er das Unternehmen auf die Existenz der Dörfer hinwies, damit es diese informieren, ihre Felder entsprechend lokalisieren und bei den Arbeiten aussparen konnte. Der settler chief von Kpachaa, der sein Land über den Tijo Naa erhalten hatte, wurde zeitnah durch den Tijo Naa informiert, während die sub-chiefs von Jashei und Jimle erst einige Zeit später durch die Investoren selbst von dem Projekt erfuhren, obwohl sie Indigene sind und damit permanente Landrechte haben ${ }^{68}$. Entsprechend konnten auch sie die Informationen erst später an die settler communities auf ihrem Land weitergeben. Der Informationsfluss wurde damit willkürlich durch den paramount chief beeinflusst und Beziehungen, die vorher für individuelle Vereinbarungen wichtig waren - zum Beispiel zu den sub-

66 Landnutzer*innen aus Nsonyameye, Baama \& Dukusen, Gruppendiskussion am 27.4.2016; Landnutzer aus Dukusen, Interview am 26.4.2016; Landnutzerin aus Nsonyameye, Interview am 26.4.2016; Landnutzerin aus Nsonyameye, Interview am 27.4.2016; Landnutzerin aus Nsonyameye, Interview am 27.4.2016.

67 Registerführer des Agogo Traditional Council und Leiter des Agogo Customary Land Secretariat, Interview am 20.4.2016; sub-chief von Baama, Interview am 20.04.2016.

68 Landnutzer*innen aus jimle und Cumani, Gruppendiskussion am 10.5.2016; Landnutzer*innen und settler-chief aus Kpachaa, Gruppendiskussion am 11.5.2016; Landnutzer"innen und sub-chief von Jashei, Gruppendiskussion am 15.5.2016. 
chiefs - konnten Zugang zu Land nicht mehr gewährleisten. Darüber hinaus sei der Großteil der Arbeitsplätze, die BioFuel Africa schuf, an Familienmitglieder, Freund*innen und Bekannte des Tijo Naas aus Tamale vergeben worden, wo der Tijo Naa seinen Hauptwohnsitz hat (Kwoyiga 2012: 46). Klientelismus gewinnt damit in beiden Fällen maßgeblich an Bedeutung.

Aufgrund des Verlusts oder der Einschränkung von Zugang setzten Landnutzer*innen in beiden Gegenden große Hoffnungen in eine Anstellung bei den Unternehmen. Lohnarbeit ermöglichte in beiden Gegenden jedoch nur einer geringen Zahl von Landnutzer*innen Zugang zu Land, der darüber hinaus nur temporär oder sehr unsicher war, da das Unternehmen Arbeitsverhältnisse jederzeit aufkündigen konnte. ScanFarm stellte insgesamt 80 Personen ein, von denen 30 permanent beschäftigte Fachkräfte waren, die nicht aus den Dörfern in der Projektgegend kamen. 50 Angestellte waren temporär beschäftigte Hilfs- und Gelegenheitsarbeiter*innen (Erntehelfer*innen, Pestizidsprüher*innen, Pflanzer*innen) aus den Dörfern, die das Unternehmen ein bis drei Monate im Jahr beschäftigte. Sie erhielten pro Tag einen Lohn von 15 bis 20 GHS, was damals ca. 9 bis 12 Euro entsprach (Boamah/Overå 2016: 122). BioFuel Africa beschäftigte während des kurzen Höhepunkts des Projekts im Jahr 2009400 Angestellte (Persönliche Kommunikation CEO BioFuel Africa). Aus den Dörfern in der Projektgegend kamen jedoch nur ca. 70-80 Personen und 50 davon alleine aus Jimle ${ }^{69}$. Der Großteil der Angestellten aus den Dörfern waren Männer, von denen die meisten saisonal als Pestizidsprüher oder Feldarbeiter arbeiteten. Einige bekamen Posten als Aufseher und Wachmänner. Die wenigen Frauen, die das Unternehmen beschäftigte, arbeiteten als Ernte- und Pflanzhelferinnen. Je nach Position erhielten sie einen Lohn von 65 bis 200 GHS pro Monat. Das entsprach zu diesem Zeitpunkt ca. 40 bis 120 Euro. Feldarbeiter*innen bekamen mit 65 GHS den geringsten Lohn, während die Aufseher mit 200 GHS pro Monat den höchsten Lohn erhiel$\operatorname{ten}^{70}$.

69 Landnutzer*innen aus Jimle und Gumani, Gruppendiskussion am 10.5.2016.

70 Landnutzer*innen aus Jimle und Cumani, Gruppendiskussion am 10.5.2016; Landnutzer und settler-chief aus Parachanayilli, Gruppendiskussion am 13.5.2016; Landnutzer*innen und sub-chief von Jashei, Gruppendiskussion am 15.5.2016. 


\subsection{Zwischenfazit}

Vor der Vergabe des Lands an die Unternehmen ScanFarm und BioFuel Africa teilte sich die Kontrolle des Zugangs zu Land in beiden Gegenden zwischen unterschiedlichen Akteuren auf. In beiden Fällen kontrollierten der paramount chief, die sub-chiefs und Landnutzer*innen mit permanenten Rechten Zugang $\mathrm{zu}$ Land. Sie konnten darüber entscheiden, wer Land wie nutzen konnte. Die Möglichkeit dieser Akteure Zugang zu Land zu kontrollieren, leitete sich aus ihrer Herkunft ab; aus der Anerkennung als Indigene, deren Vorfahren bereits aus der Gegend stammten und Land urbar gemacht oder die das Land erstmalig bestellt hatten.

Landnutzer*innen, die Zugang zu Land nicht eigenständig kontrollierten, erlangten Zugang über rechtebasierte, relationale und strukturelle Zugangsmechanismen, die in einem wechselseitigen Verhältnis zueinander standen. Rechtebasierte Zugangsmechanismen wie traditionelle Landrechte oder individuelle Vereinbarungen waren mit relationalen und strukturellen Mechanismen wie sozialer Zugehörigkeit, sozialen Beziehungen und Netzwerken verknüpft. Landnutzer*innen, die Zugang zu Land über traditionelle Rechte erlangten, konnten diese rechtebasierten Zugangsmechanismen über relationale und strukturelle Zugangsmechanismen stärken. Landnutzer*innen, die aufgrund ihrer sozialen Zugehörigkeit keine traditionellen Landrechtsansprüche geltend machen konnten, hatten die Möglichkeit, Zugang zu Land mittels relationaler und struktureller Zugangsmechanismen zu erlangen.

Mit der Vergabe des Lands an die Unternehmen veränderte sich in beiden Fällen zunächst die Kontrolle über den Zugang zu Land. Die Verhandlungen über die Vergabe des Lands führten die Unternehmen mit den paramount chiefs und ihren elders. Die sub-chiefs und Landnutzer*innen mit permanenten Landrechten erhielten nicht die Möglichkeit an den Verhandlungen teilzunehmen und Entscheidungen mitzubestimmen. Dadurch verloren sie die Kontrolle über den Zugang zu Land an die paramount chiefs. Aufgrund der Verschiebung der Kontrolle weg von den sub-chiefs und Landnutzer*innen hin zu den paramount chiefs veränderten sich auch die Möglichkeiten Zugang zu Land zu erlangen. Landnutzer*innen, die Zugang zu Land bislang über Beziehungen $\mathrm{zu}$ anderen Akteuren als den paramount chief erlangt hatten, konnten diesen im Kontext der Landvergabe nicht aufrechterhalten. Traditionelle Landrechte und individuelle Vereinbarungen verloren maßgeblich an Bedeutung und ermöglichten es Landnutzer*innen kaum noch, einen Nutzen aus dem Land zu ziehen. Stattdessen gewannen - insbesondere im Fall von ScanFarm - staat- 
lich registrierte Rechte an Relevanz. Nach der Vergabe des Lands erlangten Akteure zudem in beiden Fällen zunehmend Zugang zu Land über klientelistische Beziehungen. 



\section{Die Bedeutung von Macht für Konflikte um Zugang zu Land}

Im vorherigen Kapitel habe ich beschrieben, über welche Mechanismen Landnutzer*innen vor der Vergabe des Lands an ScanFarm und BioFuel Africa Zugang zu Land erlangt haben und inwiefern sich diese Möglichkeiten im Kontext der Landvergabe verändert haben. Anhand meiner Analyse konnte ich zeigen, dass sich die Möglichkeiten der Landnutzer*innen, Zugang zu Land aufrechtzuerhalten oder einzufordern mit der Landvergabe deutlich verringert haben. Diese Entwicklung erkläre ich über die Veränderung der Kontrolle des Zugangs zu Land. Vor der Vergabe des Lands an die Unternehmen war die Kontrolle des Zugangs zu Land dezentral zwischen unterschiedlichen Akteuren aufgeteilt. Im Zusammenhang mit der Landvergabe an ScanFarm und BioFuel Africa lässt sich eine Zentralisierung der Kontrolle des Zugangs $\mathrm{zu}$ Land beobachten. In beiden Fällen gelang es den paramount chiefs, alleinige Kontrolle über den Zugang zu Land auszuüben.

Wie ich in Kapitel 3 herausgearbeitet habe, ist in Konflikten um Land die Fähigkeit Zugang zu Land zu kontrollieren Ausdruck von Macht. Woher sich diese Macht ableitet und wie Akteure Macht ausüben, kann sich unterscheiden. Um Erklärungsansätze zum Zusammenhang zwischen dem Handeln traditioneller Autoritäten und Konflikten um Zugang zu Land entwickeln zu können, analysiere ich im Folgenden, wie es den paramount chiefs im Kontext der Landvergabe an ScanFarm und BioFuel Africa gelungen ist, Kontrolle über den Zugang zu Land auszuüben und inwiefern sein Handeln Auswirkungen auf den jeweiligen Konflikt hatte. Anknüpfend an meinen theoretischen Rahmen untersuche ich dies anhand der analytischen Kategorien sichtbare, versteckte und unsichtbare Macht. Sichtbare Macht bezeichnet die Fähigkeit, Entscheidungen zu umstrittenen Themen gegen Widerstreben durchzusetzen. Die Voraussetzung für die Analyse sichtbarer Macht ist die Kommunikation widersprüchlicher Interessen, über die ein Konflikt für Forschende be- 
obachtbar wird. Sichtbare Macht kann in diesem Konflikt in Form von Entscheidungen zu umstrittenen Themen oder Gegenständen analysiert werden. Ein deutlicher Hinweis auf die Ausübung sichtbarer Macht ist ein Befehl, der Gehorsam findet. Ein Befehl kann eine offen kommunizierte Entscheidung oder Anweisung sein. "Gehorsam finden« bedeutet, dass Gengenspieler*innen (aus welchen Gründen auch immer) sich diesem Befehl nicht widersetzen können, ihn widerstandslos akzeptieren oder sogar unterstützen, auch wenn er sich gegen ihre Interessen richtet. Versteckte Macht bezeichnet die Fähigkeit, politische und soziale Werte oder institutionelle Verfahrensweisen zu begünstigen, die dazu beitragen, dass nur solche Themen in Entscheidungsräume gelangen, die nicht im Widerspruch zu den eigenen Interessen stehen bzw. diese nicht gefährden. Versteckte Macht auszuüben bedeutet, potenziell konfliktträchtige Themen aus Entscheidungsräumen auszuschließen oder bestimmen zu können, wer an Entscheidungsprozessen teilnimmt. Auf diese Weise bekommen Gegenspieler*innen für ihre Anliegen keine Verhandlungsmöglichkeiten. Zentral für die Analyse versteckter Macht ist die Berücksichtigung vorherrschender Werte, Überzeugungen und institutioneller Verfahrensweisen, die sich zugunsten bestimmter Akteure und auf Kosten anderer auswirken. Unsichtbare Macht bezeichnet die Fähigkeit, die Einstellungen, Interessen und Bedürfnisse anderer Akteure so zu prägen, $\mathrm{zu}$ beeinflussen und schließlich zu bestimmen, dass diese Missstände und die ihnen zugrundeliegenden Widersprüche sowie Handeln entgegen der eigenen Interessen gar nicht erst wahrnehmen. Damit geht einher, dass Akteure den Status quo als selbstverständlich, unveränderbar oder sogar vorteilhaft wahrnehmen, nicht versuchen ihn zu ändern und willig das Handeln entgegen ihrer Interessen mittragen.

Im Folgenden zeige ich entlang des Verlaufs der Landvergabe, über welche Formen der Macht die paramount chiefs Kontrolle über den Zugang zu Land erlangten und aufrechterhalten konnten. Daran anschließend analysiere ich, wie die unterschiedlichen Machtformen sich gegenseitig bedingen und inwiefern ihre Ausübung die Entstehung und den Verlauf von Konflikten beeinflusst. Ich zeige, dass die Analyse des Handelns traditioneller Autoritäten entlang von sichtbaren, versteckten und unsichtbaren Machtverhältnissen wichtige Erkenntnisse in Bezug auf den Verlauf der Vergabe von Land und damit einhergehenden Konflikten zwischen Landnutzer*innen, Unternehmen und traditionellen Autoritäten liefert. 


\subsection{Kontrolle des Zugangs zu Land über sichtbare, unsichtbare und versteckte Macht}

Den Handlungsspielraum der paramount chiefs im Rahmen der großflächigen Vergabe von Land und ihre Fähigkeit, Kontrolle über den Zugang zu Land ausüben zu können, bestimmt in erster Linie ihr Selbstverständnis und ihr Auftreten als Eigentümer des gesamten durch sie verwalteten Lands. Obgleich die Verfassung von 1992 traditionellen Autoritäten im Allgemeinen - und damit auch den paramount chiefs - explizit die Rolle von Treuhändern zuschreibt (Art. 36, Abs. 8), treten sie in der Regel als Eigentümer des Landes auf. Staatliche und zivilgesellschaftliche Akteure sowie Vertreter*innen formalisierter traditioneller Institutionen kritisieren diese Haltung der chiefs und verweisen auf die damit einhergehende Reproduktion von Ungleichheitsverhältnissen ${ }^{1}$. Innerhalb ihrer Gemeinschaft genießen traditionelle Autoritäten jedoch eine große Legitimität als Landeigentümer. Landnutzer*innen, die ihren Zugang $\mathrm{zu}$ Land über traditionelle Autoritäten erlangt haben, erkennen diese aus Respekt und Loyalität als Eigentümer des Lands an (Ahmed et al. 2018), insbesondere um ihren eigenen Zugang zu Land aufrechtzuerhalten ${ }^{2}$. Aus ihrer Anerkennung als Landeigentümer leitet sich wiederum die Legitimität der chiefs als solche $\mathrm{ab}$.

In beiden untersuchten Fällen nutzten die paramount chiefs gegenüber den Unternehmen ScanFarm und BioFuel Africa ihre Position als vermeintliche Landeigentümer. Als solche war es ihnen möglich, die Verhandlungen über die Vergabe des Lands mit den Unternehmen allein zu führen ${ }^{3}$. Ihr Auftreten als Landeigentümer ermöglichte es ihnen, versteckte Macht gegenüber

Vorsitzender des National Office of the Administrator of Stool Lands, Interview am 22.3.2016; Mitarbeiterin des Regional Office of the Administrator of Stool Lands der Northern Region, Interview am 4.5.2016; Mitarbeiter des Chana Investment Promotion Center Regional Office der Northern Region, Interview am 8.3.2017; Mitarbeiterin des Environmental Protection Agency Regional Office der Northern Region, Interview am 8.3.2017; Projektleiter des Land and Resource Management Center, Interview am 12.4.2016; Research Officer des Regional House of Chiefs der Northern Region, Interview am 8.3.2017.

2 Landnutzer*innen aus jimle und Gumani, Gruppendiskussion am 10.5.2016.

3 Geschäftsführer ScanFarm, Interview am 4.4.2016; Ehemaliges Vorstandsmitglied von ScanFarm, Interview am 6.5.2016; Leitender Angestellter BioFuel Africa, Interview am 24.5.2016. 
den Landnutzer*innen und sub-chiefs auszuüben. Durch ihr Handeln begünstigten die paramount chiefs gesellschaftliche Werte und institutionelle Praktiken, die vorteilhaft für die Durchsetzung ihrer eigenen Interessen waren. Damit handelten sie entgegen ihrer verfassungsrechtlichen Rolle als Treuhänder des gemeinschaftlichen Lands. Die Ausübung versteckter Macht hatte zur Folge, dass Akteure mit gegensätzlichen Interessen wie die subchiefs und Landnutzer*innen nicht die Möglichkeit bekamen, diese öffentlich $\mathrm{zu}$ vertreten und $\mathrm{zu}$ verteidigen. Laut staatlichem Recht muss der Vergabe von Land der gesamte traditional council zustimmen ${ }^{4}$. Diesem gehören neben dem paramount chief als Vorsitzendem des traditional council die sub-chiefs, Village-chiefs und elders (Berater*innen) an. Sie bilden gemeinsam einen Kontrollmechanismus, der sicherstellen soll, dass die Interessen aller Beteiligten vertreten werden, so wie es die National Land Policy von 1999 fordert (Ministry of Lands and Forestry 1999). In der Praxis beziehen die paramount chiefs jedoch oftmals weder den gesamten traditional council in die Verhandlungen mit Investoren ein, noch informieren sie die übrigen Mitglieder der Gemeinschaft über das Vorhaben (Nolte/Väth 2015: 73f.). Die Abläufe der Landvergabe an ScanFarm und BioFuel Africa sind typische Beispiele dafür. Nach der Handlungstypologie Max Webers (1972 [1922]: 12) kann das Handeln der paramount chiefs als zweckrational oder traditional verstanden werden. Zweckrationales Handeln ist mit einem bestimmten Ziel - in diesem Fall dem Ausschluss der sub-chiefs und Landnutzer*innen aus den Verhandlungen - verbunden. Traditionales Handeln beruht auf Gewohnheit. Unabhängig davon, ob das Handeln der paramount chiefs zweckrational oder traditional war, kann es analytisch als Ausübung versteckter Macht verstanden werden. Es führte dazu, dass Akteure mit potenziell widersprüchlichen Interessen aus Entscheidungsräumen ausgeschlossen wurden, ohne dass sich daraus (negative) Konsequenzen für die paramount chiefs ergaben. Die Entscheidung im Vorfeld der Verhandlungen keine Rechenschaft gegenüber Landnutzer*innen oder sub-chiefs abzulegen, sie nicht in die Landvergabe miteinzubeziehen oder sie diesbezüglich zu konsultieren, hatte keinerlei Folgen für die paramount chiefs. Die Landnutzer*innen und sub-chiefs beschränkte das Handeln der paramount chiefs hingegen maßgeblich in ihrem Handlungsspielraum.

Mit den Vorsitzenden von ScanFarm führte der paramount chief die Verhandlungen über die Landvergabe an das Unternehmen von Beginn und bis 
zur Zusicherung des Pachtvertrags allein. Nur einige elders des ATC waren als Berater*innen beteiligt ${ }^{5}$ Bei ihnen handelte es sich jedoch nicht um die sub-chiefs der betroffenen Dörfer. Landnutzer*innen und sub-chiefs informierte der paramount chief nicht über sein Vorhaben, ihr Land an ScanFarm zu verpachten ${ }^{6}$. Später, als der Fall zivilgesellschaftliche und mediale Aufmerksamkeit auf sich zog (ActionAid/FoodSPAN 2010; Bull 2010; Dogbevi 23.02.2010) und zahlreiche Landnutzer*innen sowie engagierte Bewohner*innen aus der Umgebung gegen das Vorhaben demonstrierten, rechtfertigte der paramount chief sein Handeln mit dem Argument, sein Ziel sei es gewesen, nur das durch ihn persönlich verwaltete, ungenutzte stool land zu verpachten und nicht das Land der Landnutzer"innen ${ }^{7}$. Eine offizielle Karte des gepachteten Lands, die das Unternehmen im Rahmen der Registrierung des Pachtvertrags bei der Lands Commission anfertigen ließ und welcher der traditional council unter dem Vorsitz des paramount chiefs zugestimmt hatte, schloss jedoch unter anderem das Land von Dukusen, Nsonyameye und Baamaa mit ein. Durch den Ausschluss der Landnutzer*innen und sub-chiefs aus den Verhandlungen und die fehlende Weitergabe von Informationen bezüglich des Vorhabens konnten diese jedoch nicht wissen, dass sie den Zugang zu ihrem Land verlieren würden. Dementsprechend waren sie nicht in der Lage zu erkennen, dass der paramount chief Entscheidungen gegen ihre Interessen und ihren Willen traf. Ohne das Wissen, dass der paramount chief mit dem Unternehmen über die Vergabe ihres Lands verhandelte, konnten sie weder die Teilnahme an diesen Verhandlungen einfordern noch Widerstand gegen die Vergabe des Lands leisten. Die Ausübung versteckter Macht führte somit zu einem absoluten Ausschluss der Landnutzer*innen und sub-chiefs aus den Verhandlungen

Geschäftsführer ScanFarm, Interview am 4.4.2016; Registerführer des Agogo Traditional Council und Leiter des Agogo Customary Land Secretariat, Interview am 20.4.2016.

6 Sub-chief von Dukusen, Interview am 19.4.2016; sub-chief von Nsonyameye, Interview am 20.4.2016; sub-chief von Baama, Interview am 20.04.2016; Landnutzerin aus Nsonyameye, Interview am 24.4.2016; Landnutzerin aus Nsonyameye, Interview am 24.4.2016; Landnutzer aus Nsonyameye, Interview am 25.4.2016; Landnutzerin aus Nsonyameye, Interview am 27.4.2016; Landnutzerer aus Nsonyameye und Mitglieder der Agogoman mma kuo, Interview am 16.3.2017; Landnutzerin aus Dukusen, Interview am 16.3.2017.

7 Sub-chief von Dukusen, Interview am 19.4.2016; sub-chief von Nsonyameye, Interview am 20.4.2016; Registerführer des Agogo Traditional Council und Leiter des Agogo Customary Land Secretariat, Interview am 20.4.2016; Landnutzerin aus Nsonyameye, Interview am 24.4.2016; 1 Landnutzer aus Nsonyameye, Interview am 25.4.2016. 
um die Landvergabe und ermöglichte dem paramount chief, alleinige Kontrolle über den Zugang zu Land auszuüben.

Die Verhandlung der Landvergabe an BioFuel Africa fand ebenfalls zunächst bilateral zwischen dem paramount chief - dem Tijo Naa - und den Geschäftsführern des Unternehmens statt. Auch hier war das Auftreten des paramount chiefs als Landeigentümer zentral dafür, dass sich das Unternehmen mit seinem Anliegen zunächst an ihn allein wandte, um die Verhandlungen über die Landvergabe $\mathrm{zu}$ initiieren ${ }^{8}$. Anders als ScanFarm forderten die Vorsitzenden von BioFuel Africa jedoch ein Treffen mit allen von der Landvergabe betroffenen Akteuren ein, um über ihr Vorhaben zu informieren (Tsikata/Yaro 2011: 20). Das Unternehmen organisierte daraufhin ein Treffen, das im Tamale Cultural Center stattfand. An diesem Treffen nahmen der paramount chief und seine elders, einige sub-chiefs und Landnutzer*innen aus der Gegend, Angestellte der District Assembly und im Landsektor engagierte NGOs teil (ebd.). Ziel des Treffens war es, allen betroffenen Akteuren das Vorhaben vorzustellen und ihnen die Möglichkeit zu geben, Bedenken $\mathrm{zu}$ äußern (Tsikata/Yaro 2011). Es ging jedoch nicht darum, die von der Landvergabe betroffenen Akteure in die Entscheidung der Landvergabe miteinzubeziehen. Das hätte bedeutet, ihnen die Möglichkeit zu geben, sich gegen das Vorhaben auszusprechen. Die Ablehnung des Vorhabens durch Teilnehmende an dem Treffen war auf der Agenda jedoch nicht vorgesehen. Der paramount chief und die Geschäftsführer des Unternehmens fragten die sub-chiefs und Landnutzer*innen nicht nach ihrer Zustimmung zu dem Vorhaben, sondern setzten diese - aufgrund der vermeintlichen Vorteile, die sich durch das Projekt ergeben sollen - voraus. Damit sprach er ihnen faktisch die Kontrolle über den Zugang zu Land ab.

Auch wenn der paramount chief und die Geschäftsführer von BioFuel Africa die Zustimmung der Landnutzer*innen und sub-chiefs zu der Landvergabe voraussetzten und ihnen dadurch die Kontrolle über den Zugang zu Land entzogen, war die Teilnahme an dem Treffen für die Möglichkeiten des weiteren Zugangs zu Land relevant. Das stellte sich später in Bezug auf die Umsetzung des Projekts und die Durchführung der Umwelt- und Sozialverträglichkeitsstudie heraus, wie ich weiter unten erläutere. Alle Landnutzer*innen bekamen jedoch nicht die Möglichkeiten an dem Treffen teilzunehmen. Neben 
staatlichen und zivilgesellschaftlichen Akteuren lud das Unternehmen, beruhend auf den Informationen, die ihnen der paramount chief gab, lediglich Vertreter*innen aus den Dörfern Jimle, Jashie, Tuya und Kpachaa ein ${ }^{9}$. Indem er die Bewohner*innen von Parachanayilli und Changolinaaya von dem Treffen ausschloss, übte der paramount chief erneut versteckte Macht aus. Jimle, Jashie und Tuya sind indigene Gemeinschaften. Kpachaa ist eine settler community, ein Dorf in dem ausschließlich migrantische Landnutzer*innen leben. Die Bewohner*innen von Kpachaa sind Anfang der $2000 e r$ Jahre aus der Umgebung von Tamale gekommen und haben ihr Land durch den paramount chief erhalten ${ }^{10}$. Andere settler communities wie Parachanayilli und Changolinaaya erhielten ihren Zugang zu Land durch den sub-chief von Jashie. Zu dem Informationstreffen in Tamale lud das Unternehmen nur die sub-chiefs und Landnutzer*innen, denen der paramount chief Zugang zu Land gewährte ${ }^{11}$. Die Bewohner*innen und sub-chiefs der anderen Dörfer wurden im Nachhinein teilweise durch den sub-chief von Jashie und teilweise durch den Tijo Naa informiert. Sie leiteten jedoch nicht alle Informationen verlässlich weiter ${ }^{12}$, was auf unklare Zuständigkeiten zurückzuführen ist. Der sub-chief von Jashie erachtete es als Aufgabe des Tijo Naa, alle Landnutzer*innen in den betroffenen Dörfern und settler communities über das Vorhaben von BioFuel Africa zu informieren. Er habe über die Vergabe des Lands entschieden und sei daher gemeinsam mit dem Unternehmen dafür zuständig, alle betroffenen Landnutzer*innen darüber in Kenntnis zu setzen ${ }^{13}$. Die elders des paramount chiefs hingegen kritisierten an dieser Haltung des sub-chiefs von Jashie, dass der paramount chief nicht über die Anwesenheit aller settler communities informiert gewesen sei, da er das Land nicht an sie vergeben habe ${ }^{14}$. Die Konsequenz der

Landnutzer*innen aus Jimle und Gumani, Gruppendiskussion am 10.5.2016; Landnutzer*innen und settler-chief aus Kpachaa, Gruppendiskussion am 11.5.2016; Landnutzer*innen und sub-chief von Tuya, Gruppendiskussion am 14.5.2016; Landnutzer*innen und sub-chief von Jashei, Gruppendiskussion am 15.5.2016. Landnutzer*innen und settler-chief aus Kpachaa, Gruppendiskussion am 11.5.2016. Landnutzer*innen aus Nsonyameye, Baama \& Dukusen, Cruppendiskussion am 27.4.2016; Landnutzer und settler-chief aus Parachanayilli, Gruppendiskussion am 13.5.2016. Landnutzer*innen aus Nsonyameye, Baama \& Dukusen, Cruppendiskussion am 27.4.2016; Landnutzer und settler-chief aus Parachanayilli, Gruppendiskussion am 13.5.2016. Paramount-chief und elders von Tijo, Gruppendiskussion am 8.5.2016. 
vermeintlichen Unkenntnis des Tijo Naa war, dass die späteren Studien, die im Rahmen des Environmental and Social Impact Assessment durchgeführt wurden, diese Dörfer ebenfalls nicht berücksichtigten (Bruce 2008; SGS Environment 2009). Das wiederum hatte Auswirkungen auf ihren Zugang zu Land. In den beiden settler communities Parachanayilli und Changolinaaya zerstörte das Unternehmen durch die Arbeiten auf dem Land Felder von Landnutzer*innen, stellte ihnen jedoch keine alternativen Landflächen zu Verfügung. Dieses Vorgehen unterschied Parachanayilli und Changolinaaya von den Dörfern, dessen sub-chiefs bei dem Treffen mit dem Unternehmen anwesend waren. Dort achtete BioFuel Africa darauf, bei der Rodung und dem Umpflügen für die Jatropha-Plantage die Felder der Landnutzer*innen auszusparen oder bot ihnen an, neue Felder außerhalb der Plantage für den Anbau vorzubereiten $^{15}$. Unabhängig davon ob das Handeln des paramount chiefs intentional war oder auf unklare Zuständigkeiten zurückzuführen ist, übte er darüber versteckte Macht aus. Sein Handeln führte dazu, dass Akteure keine oder nur unvollständige Informationen über die Landvergabe erhielten, aus Entscheidungsräumen ausgeschlossen wurden oder nicht die Möglichkeit hatten, für sie relevante Themen zu verhandeln und für ihre Interessen einzutreten.

Auch der weitere Verlauf der bilateralen Verhandlungen zwischen den Geschäftsführern von ScanFarm und dem ATC zeigt, dass die Ausübung versteckter Macht über den offensichtlichen Ausschluss der sub-chiefs und Landnutzer*innen hinausging. Neben der alleinigen Verhandlung der Landvergabe übte der paramount chief ebenfalls versteckte Macht aus, indem er den verbreiteten Brauch des drink money neu interpretierte.

Die Zahlung von drink money, von kleineren Geldbeträgen an traditionelle Autoritäten im Rahmen ihrer Konsultierung, hat in jüngster Zeit einen vermeintlich alten Brauch ersetzt. Diesem Brauch nach brachte jede Person, die traditionellen Autoritäten ein Anliegen unterbreiten wollte, diesen als Zeichen des Respekts und der Treue eine Flasche Schnaps oder einige Kolanüsse mit. Mittlerweile ist es üblich, Schnaps oder Kolanüsse durch kleinere Geldbeträge $\mathrm{zu}$ ersetzen. Bezug nehmend auf den alten Brauch ist dieses Geld allgemein als drink money bekannt ${ }^{16}$. Mit steigendem Interesse internationaler Investoren an Land und der Zunahme großflächiger Landvergaben verlangen traditionelle Autoritäten seit einigen Jahren von ihren Verhandlungspartner*innen

15 Landnutzer aus Changolinaaya, Interview am 12.5.2016; Landnutzer und settler-chief aus Parachanayilli, Gruppendiskussion am 13.5.2016.

16 Mitarbeiter des Asantehenes Land Secretariat, Interview am 10.3.2017. 
zunehmend hohe Geldbeträge dafür, dass sie ihnen Zugang zu Land ermöglichen ${ }^{17}$. Indem sie diese als drink money bezeichnen, legitimieren sie ihren alleinigen Anspruch darauf. Faktisch handelt es sich jedoch um einen Teil der Pachteinnahmen, denn mit der Zahlung einer hohen Summe an drink money gehen im späteren Verlauf niederigere Pachteinnahmen einher (Blocher 2006; Schoneveld/German 2014). Die Höhe des drink money ist eine Frage der Verhandlung zwischen Investoren und traditionellen Autoritäten. Sie ist abhängig von der Größe des Lands sowie von individuellen Entscheidungen. Daher unterscheidet sie sich von Fall zu Fall. In der Regel wird die Höhe des gezahlten drink money nicht schriftlich festgehalten und ist meistens nur den verhandelnden Parteien bekannt. Mit der Bezeichnung der Zahlungen als drink money suggerieren traditionelle Autoritäten ihren Verhandlungspartner*innen, es handele sich um eine gängige Gepflogenheit, die unumgänglich für den erfolgreichen Verlauf der Verhandlungen sei. Unklar bleibt den Investoren jedoch oftmals, dass die Zahlung von drink money nicht spätere Pacht- oder Entschädigungszahlungen ersetzt. Diese fallen an, wenn Pachtverträge über gewohnheitsrechtliches Land, die in erster Instanz mit traditionellen Autoritäten ausgehandelt wurden, bei der Lands Commission registriert werden sollen. Anders als drink money hält die Lands Commission diese Beträge schriftlich fest. Pachtzahlungen sind - wenn auch durch die Lands Commission dokumentiert - ebenfalls Gegenstand von Verhandlungen zwischen den Pächter*innen und den Empfänger*innen dieser Leistungen. Die Unterlagen der Lands Commission bilden die Grundlage für die Umverteilung der Einnahmen durch das Office oft he Administrator of Stool Lands nach dem staatlich festgelegten Umverteilungsschema. Laut diesem Umverteilungsschema stehen 55 Prozent der Einnahmen aus der Vergabe von traditionell verwaltetem Land der District Assembly zu, 20 Prozent erhält der chief, der das Land vergibt und 25 Prozent der stool, der in diesem Fall die gesamte landnutzende Gemeinschaft umfasst ${ }^{18}$. Entschädigungszahlungen stehen den vorherigen Landnutzer*innen für den Verlust ihres Lands oder ihrer Ernte zu. Über die Bezeichnung der im Zuge der Verhandlung geleisteten Zahlung als drink money haben traditionelle $\mathrm{Au}$ toritäten einen Weg gefunden, die Verteilung der Einnahmen aus der Vergabe 22.3.2016; Mitarbeiter des Asantehenes Land Secretariat, Interview am 10.3.2017. des Regional Office of the Administrator of Stool Lands der Ashanti Region, Interview am 14.04.2016.
} 
von Land, wie sie die Verfassung vorsieht, zu umgehen und den Großteil der Einnahmen für sich zu beanspruchen ${ }^{19}$.

Der Ablauf der Verhandlungen um die Landvergabe zwischen ScanFarm und dem ATC veranschaulicht diese Entwicklung beispielhaft. Um Zugang zu Land zu erlangen und einen Pachtvertrag abzuschließen, zahlte ScanFarm drink money an den $A T C^{20}$. Die genaue Höhe des drink money kennen nur die Vorsitzenden von ScanFarm, der paramount chief sowie seine engsten Vertrauten. Von ihnen war im Rahmen der Interviews jedoch niemand bereit, die genaue Summe zu benennen. Die Höhe der Zahlung ist jedoch irrelevant, um zu verstehen, inwiefern die Neuinterpretation von drink money als Ausübung versteckter Macht analysiert werden kann. Die Entscheidung des paramount chiefs, den Pachtvertrag gegen die Zahlung von drink money und nicht gegen eine offiziell dokumentierte Pachtzahlung zu gewähren, ermöglichte es ihm, Akteure wie sub-chiefs, Landnutzer*innen und District Assembly von der finanziellen Teilhabe an der Vergabe des Lands auszuschließen und die Einnahmen für sich zu behalten. Darüber hinaus wirkte sich dieses Vorgehen später auf die Möglichkeiten der Landnutzer*innen, angemessene Entschädigungszahlungen für verlorenes Land zu fordern, aus. Für die Vorsitzenden von ScanFarm bildete die Zahlung des drink money und die darauffolgende Einwilligung des paramount chiefs in die Landvergabe die Legitimationsgrundlage für die Nutzung des Lands. Dementsprechend sah sich das Unternehmen auch nicht in der Pflicht, die sub-chiefs und Landnutzer*innen einzeln über die Nutzung des Lands zu informieren oder sie für den Verlust ihres Lands zu entschädigen. Die Verantwortung dafür überließ ScanFarm dem ATC ${ }^{21}$. Über die Neuinterpretation institutioneller Verfahrensweisen sowie vorherrschender Regeln und Normen - also die Ausübung versteckter Macht - konnte sich der paramount chief selbst bereichern und seine eigenen Interessen durchsetzen.

Der Ausschluss der sub-chiefs und Landnutzer*innen aus den Verhandlungen der Landvergabe führte dazu, dass sie das Handeln des paramount chiefs entgegen ihrer Interessen nicht realisierten und daher zunächst auch gar kein Konflikt entstand. Erst die physische Anwesenheit ScanFarms auf dem Land und der Beginn der Bearbeitung des Lands Anfang 2009 hatten

19 Vorsitzender des National Office of the Administrator of Stool Lands, Interview am 22.3.2016.

20 Geschäftsführer ScanFarm, Interview am 4.4.2016; Registerführer des Agogo Traditional Council und Leiter des Agogo Customary Land Secretariat, Interview am 20.4.2016.

21 Geschäftsführer ScanFarm, Interview am 4.4.2016. 
zur Folge, dass die sub-chiefs und Landnutzer*innen der betroffenen Dörfer von dem Vorhaben erfuhren ${ }^{22}$. Als die sub-chiefs und Landnutzer*innen feststellten, dass das Land, zu dem sie bisher Zugang hatten, Teil des Pachtvertrags ist, den ScanFarm mit dem ATC abgeschlossen hatte, wandten sie sich zur Klärung der Angelegenheit an den paramount chief ${ }^{23}$. Durch die offene Kommunikation ihres Missfallens wurde der Konflikt manifest und für Außenstehende beobachtbar. Nach Gewohnheitsrecht haben Landnutzer*innen mit permanenten Landrechten ein Mitspracherecht bezüglich Entscheidungen, die ihr Land betreffen. Die Ausübung versteckter Macht über die bilateralen Verhandlungen des Pachtvertrags zwischen ScanFarm und dem ATC, die damit verbundene Kontrolle von Informationen und die Einflussnahme auf institutionelle Abläufe führte jedoch dazu, dass sie von diesem Mitspracherecht keinen Gebrauch machen konnten. Als Landnutzer*innen und subchiefs Anfang 2009 von der Landvergabe erfuhren, forderten sie dieses Mitspracherecht ein, indem sie sich an den paramount chief wandten ${ }^{24}$. Dieser wies jedoch die Verantwortung für das Handeln des Unternehmens von sich, indem er versicherte, das Land der Landnutzer*innen nicht an das Unternehmen vergeben zu haben ${ }^{25}$. Auf diese Weise zog er sich aus der Verantwortung und wich der Konfrontation aus. Statt Verantwortung für sein Handeln zu übernehmen und die vermeintlich fälschliche Nutzung des Lands durch das Unternehmen mit ScanFarm aufzuklären, riet er den sub-chiefs und Landnutzer*innen dazu, das Gespräch mit ScanFarm zu suchen. Da sich das Projekt bereits in der Umsetzungsphase befände und die Farmen der Landnutzer* innen zerstört seien, rate er ihnen, dem Unternehmen ihr Land gegen eine Entschädigungszahlung zu überlassen. Schließlich brächte das Projekt auf lange

22 Landnutzerin aus Nsonyameye, Interview am 24.4.2016; Landnutzer aus Nsonyameye, Interview am 25.4.2016; Landnutzerer aus Nsonyameye und Mitglieder der Agogoman mma kuo, Interview am 16.3.2017.

23 Landnutzerin aus Nsonyameye, Interview am 27.4.2016; Landnutzer aus Nsonyameye, Interview am 28.4.2016; Landnutzerer aus Nsonyameye und Mitglieder der Agogoman mma kuo, Interview am 16.3.2017.

24 Landnutzerin aus Nsonyameye, Interview am 27.4.2016; Landnutzerin aus Nsonyameye, Interview am 27.4.2016; Landnutzer aus Nsonyameye, Interview am 28.4.2016; Landnutzerer aus Nsonyameye und Mitglieder der Agogoman mma kuo, Interview am 16.3.2017.

25 Sub-chief von Nsonyameye, Interview am 20.4.2016; Landnutzerin aus Nsonyameye, Interview am 27.4.2016. 
Sicht Entwicklung in die Gegend, die allen zugutekäme ${ }^{26}$. Die sub-chiefs und einen großen Teil der Landnutzer*innen überzeugte er von diesem Vorgehen, indem er ihnen die vermeintlichen Vorteile darlegte, die durch die Anwesenheit von ScanFarm entstünden. Dazu zählten der Bau einer Schule und eines Krankenhauses, die Verbesserung des Wasserzugangs und der Infrastruktur sowie die Verbesserung der ökonomischen Lage durch die Schaffung von Arbeitsplätzen ${ }^{27}$. Indem der paramount chief die sub-chiefs und Landnutzer*innen eigenverantwortlich mit dem Unternehmen verhandeln ließ, suggerierte er ihnen, sie hätten ein Mitspracherecht. Einige Landnutzer*innen sprachen daraufhin persönlich mit dem Geschäftsführer von ScanFarm, um über die weitere Nutzung ihres Lands zu verhandeln. Insgesamt 46 der mehr als 200 Landnutzer*innen, von denen viele bereits zuvor in der Vereinigung Agogoman mma kuo (weltweite Bürger*innen von Agogo) organisiert waren, forderten ihr Land von ScanFarm zurück ${ }^{28}$. Sie erhoben Widerspruch gegen die Vergabe ihres Lands in Form von Briefen oder versuchten das Unternehmen mit ihrer physischen Präsenz auf dem Land sowie der Androhung von Waffengewalt an der Fortsetzung ihrer Arbeit zu hindern ${ }^{29}$. Im Juli 2009 bemühte sich das Unternehmen mit Unterstützung des paramount chiefs dennoch ohne weitere Konsultierung der Landnutzer*innen und sub-chiefs um die Registrierung des Pachtvertrags bei der staatlichen Lands Commisson, um das Vorhaben offiziell abzusichern ${ }^{30}$. Zwei Monate später, im September 2009, wies die Lands Commission das Unternehmen auf die Notwendigkeit hin, den Lageplan für die Registrierung des Lands zu ändern, da Teile des Lands bereits auf andere Nutzer*innen registriert seien. Demnach habe bereits 1987 der benachbarte

26 Sub-chief von Dukusen, Interview am 19.4.2016; sub-chief von Nsonyameye, Interview am 20.4.2016.

27 Sub-chief von Dukusen, Interview am 19.4.2016; Leiter des Department of Agriculture des Asante Akyem North District, Interview am 22.4.2016.

28 Landnutzerer aus Nsonyameye und Mitglieder der Agogoman mma kuo, Interview am 16.3.2017; Landnutzer aus Nsonyameye und Vorsitzender der Agogoman mma kuo, Interview am 16.3.2017.

29 Landnutzerer aus Nsonyameye und Mitglieder der Agogoman mma kuo, Interview am 16.3.2017.

30 Brief des lands officer der Regional Lands Commission (Ashanti Region) an Nana Akuoku Sparpong (paramount chief des ATC), 3.9.2009. 
Kumawu Stool Land für die Dauer von 50 Jahren an Jankoff Farms Ltd vergeben. Einen weiteren Teil habe der Agogo Stool an eine Einzelperson verpachtet ${ }^{31}$.

In Registrierungsverfahren von Pachtverträgen ist eine Aufgabe der Lands Commission zu prüfen, ob das entsprechende Land bereits auf andere Personen registriert ist. Sie berücksichtigt jedoch nur staatliche Registrierungen und nicht bestehende traditionelle Landrechte ${ }^{32}$. Für die Erfassung traditioneller Rechte sind traditionelle Autoritäten zuständig. Im Kontext der Landvergabe an ScanFarm waren dies die sub-chiefs, die Kenntnisse über die Nutzung von Land in ihrem Zuständigkeitsbereich haben. Da die sub-chiefs jedoch nicht in die Verhandlungen miteingebunden waren, berücksichtigte die Lands Commission diese Informationen im Rahmen der Registrierung des Pachtvertrags zunächst nicht. Als vermeintlicher Landeigentümer stellte der paramount chief dem Unternehmen die nötige Einwilligung aus, um den Pachtvertrag offiziell registrieren zu lassen ${ }^{33}$. Damit handelte er entgegen des Ablaufs, den staatliches Recht bezüglich der großflächigen Vergabe von Land vorsieht (Ministry of Lands and Forestry 1999). Demnach sollten der paramount chief oder das Unternehmen Behörden wie die Lands Commission und die EPA zu Beginn der Verhandlungen der Landvergabe und insbesondere vor der Umsetzung von Vorhaben auf dem Land informieren, damit diese einen Austausch zwischen allen beteiligten Akteuren initiieren können ${ }^{34}$. Ziel dieses Austauschs ist es, $\mathrm{zu}$ evaluieren, ob alle Akteure gleichermaßen über das Vorhaben informiert sind, mögliche Auswirkungen des Vorhabens darzulegen und im Gespräch weitere Folgen sowie mögliche Maßnahmen, um diesen entgegenzuwirken, $\mathrm{zu}$ ermitteln. In diesem Rahmen sollen sich die beteiligten Akteure gegebenenfalls auch auf angemessene Entschädigungszahlungen einigen. Schließlich soll der Austausch Raum für Einwände, Fra-

31 Brief des lands officer der Regional Lands Commission (Ashanti Region) an Nana Akuoku Sparpong (paramount chief des ATC), 3.9.2009.

Abteilungsleiterin der Regional Lands Commission der Ashanti Region, Interview am 13.04.2016.

33 Geschäftsführer ScanFarm, Interview am 4.4.2016.

34 Vorsitzender der National Lands Commission, Interview am 13.11.2015; Abteilungsleiterin der Regional Lands Commission der Ashanti Region, Interview am 13.04.2016; Abteilungsleiter, Valuation Division, Regional Lands Commission der Northern Region, Interview am 4.5.2016; Abteilungsleiter, Survey \& Mapping Division, Regional Lands Commission der Northern Region, Interview am 5.5.2016; I-StA-12; Mitarbeiterin des Environmental Protection Agency Regional Office der Northern Region, Interview am 8.3.2017. 
gen und Widerspruch bieten ${ }^{35}$. An dieses Vorgehen hielt sich ScanFarm jedoch nicht. Mit dem Einverständnis des paramount chiefs begann das Unternehmen bereits ohne die Bestätigung des Pachtvertrags durch die Lands Commission, das Land zu bearbeiten. Auch auf diese Weise bestimmte er institutionelle Verfahrensweisen.

Zu Beginn der Verhandlung um die Landvergabe an ScanFarm sicherte sich der paramount chief die Kontrolle über den Zugang zu Land, indem er institutionelle Verfahrensweisen neu interpretierte und somit versteckte Macht ausübte. Auf diese Weise nahm er den sub-chiefs und Landnutzer*innen die Möglichkeit der Teilnahme an der Verhandlung oder des Widerstands gegen die Vergabe des Lands. Mit der Bearbeitung des Lands durch ScanFarm handelte der paramount chief erstmals offen gegen den Willen der Landnutzer* innen und sub-chiefs, was als Ausübung sichtbarer Macht verstanden werden kann. Da die Verhandlung der Landvergabe bisher bilateral zwischen dem Unternehmen und dem paramount chief stattgefunden hatten, vermied ScanFarm zunächst den direkten Dialog, den sub-chiefs und Landnutzer*innen initiierten. Stattdessen wandte sich das Unternehmen an den paramount chief, um ihn über den Widerstand der Landnutzer*innen gegen die Bearbeitung des Lands zu informieren ${ }^{36}$. Die darauffolgende Reaktion des paramount chiefs weist erstmals auf die Ausübung sichtbarer Macht hin, also auf die Durchsetzung des eigenen Willens gegen Widerstreben. Auf den Bericht des Unternehmens über den Widerstand einiger Landnutzer*innen antwortete der Registrar des ATC im Januar 2010 mit einem Brief, in dem er ScanFarm explizit die Befugnis erteilte, auch im Fall von Widerstand weiterhin auf dem Land arbeiten zu dürfen. Sollte es zu weiteren Zwischenfällen mit den Landnutzer*innen kommen, würde sich der Agogo Stool - unter dem Vorsitz des paramount chiefs - der Angelegenheit annehmen. Der Brief hatte folgenden Wortlaut: "By this grant of authority, the Company is to go about its clearing and farming activities and that if there happens to be any protestor(s) the matter would be addressed by the Agogo Stool ${ }^{37}$. Damit legitimierte er das Handeln des Unternehmens und wandte sich wissentlich gegen den zuvor ihm und dem Unternehmen gegenüber kommunizierten Willen der Landnutzer"innen. Auf diese Weise übte

\footnotetext{
35 Abteilungsleiterin der Regional Lands Commission der Ashanti Region, Interview am 13.04.2016; I-StA-12; Mitarbeiter des Chana Investment Promotion Center National Office, Interview am 21.3.2017.

36 Ceschäftsführer ScanFarm, Interview am 4.4.2016.

37 Brief des Registerführers des ATC an den Ceschäftsführer von ScanFarm, 4.1.2010.
} 
er sichtbare Macht über sie aus. Er missachtete seine Rolle als Treuhänder des gemeinschaftlichen Lands, machte die im Vorfeld für sich beanspruchte Kontrolle über den Zugang zu Land weiterhin geltend und beschränkte den Zugang der Landnutzer*innen zu ihrem Land. Bis zu diesem Zeitpunkt hätte sein Handeln noch als traditionales, auf Gewohnheit beruhendes Handeln verstanden werden können, das nicht notwendigerweise zweckrational ist. Wie der Brief verdeutlicht, handelte er nun klar intentional gegen den Willen der Landnutzer*innen. Auch erteilte er dem Unternehmen die Erlaubnis, das Land gegen den Willen der Landnutzer*innen zu bearbeiten, obwohl der Pachtvertrag noch nicht durch die Lands Commission registriert war ${ }^{38}$. Vor der Registrierung hätte ScanFarm nicht mit der Bearbeitung des Lands beginnen dürfen.

Maßnahmen, die von staatlicher Seite aus vorgesehen sind und im Rahmen der Registrierung des Pachtvertrags stattfinden sollen, wurden somit erst umgesetzt, nachdem einige Landnutzer*innen den Zugang zu ihrem Land bereits verloren hatten. Dies wirkte sich maßgeblich auf die Handlungsmöglichkeiten von sub-chiefs und Landnutzer*innen im weiteren Verlauf der Landvergabe aus. Die Bearbeitung des Lands durch ScanFarm und damit einhergehende Folgen wie die Zerstörung der Ernten, von Grenzmarkierungen und Zugangswegen bedeuteten für die sub-chiefs und Landnutzer*innen den Verlust von Zugang zu ihrem Land. Der Verlust von Zugang wiederum beeinflusste die Verhandlungsposition der sub-chiefs und Landnutzer*innen gegenüber dem Unternehmen. Landnutzer*innen, die ihr Land vor der Landvergabe verpachtet hatten und darüber ein Einkommen erwirtschafteten, mussten aufgrund der Arbeiten des Unternehmens auf dem Land ihren Pächter*innen die Pacht rückerstatten, ohne jedoch selbst einen Nutzen aus dem Land ziehen zu können (Boamah 2015: 25). Subsistenzwirtschaft betreibende Landnutzer*innen bemühten sich aufgrund des Verlusts von Zugang $\mathrm{zu}$ ihrem Land um eine Alternative und pachteten andernorts Felder, was ebenfalls mit unerwarteten Kosten verbunden war (ebd.). Für Landnutzer*innen, die ihre Ernte durch die Arbeiten des Unternehmens verloren hatten, war es schwierig eine Entschädigung für diesen Verlust zu erwirken, da sie nicht beweisen konnten, dass sie das Land zuvor bestellt hatten ${ }^{39}$. Durch den

38 Brief des lands officer der Regional Lands Commission (Ashanti Region) an Nana Akuoku Sparpong (paramount chief des ATC), 3.9.2009; Brief des Registerführers des ATC an den Ceschäftsführer von ScanFarm, 4.1.2010.

39 Landnutzer aus Nsonyameye, Interview am 28.4.2016. 
fehlenden Zugang zu ihrem Land befanden sich all diese Landnutzer*innen in einer prekären Lage, die durch den Verlust von Einkommensmöglichkeiten und mitunter ihrer Lebensgrundlage gekennzeichnet war. Um diese Situation zu bewältigen, war ihre einzige Handlungsoption, eine alternative Einkommensquelle zu finden. Zur Absicherung ihres Lebensunterhalts willigte ein Großteil der Landnutzer*innen daher rückwirkend in die Vergabe des Lands ein, unter der Bedingung, Entschädigungszahlungen für den Verlust des Lands zu erhalten. Interviewpartner*innen berichteten davon, keine andere Wahl gehabt zu haben, als im Nachhinein in die Landvergabe einzuwilligen, da das Land de facto bereits an ScanFarm vergeben gewesen sei, durch das Unternehmen bearbeitet wurde und sie daher keinen Zugang mehr dazu hatten ${ }^{40}$.

In dem durch die Lands Commission und die EPA initiierten Gespräch, das im Januar 2010 im Palast des paramount chiefs zwischen sub-chiefs, Landnutzer*innen und dem Unternehmen stattfand, ging es der Situation entsprechend insbesondere darum, die Höhe der Entschädigungszahlungen festzusetzen. Ziel des Gesprächs war es nicht, die Landvergabe per se zu verhandeln. Aufgrund der zuvor durch ScanFarm geleisteten Zahlung von drink money war das Unternehmen jedoch nur bereit, sehr geringe Entschädigungszahlungen zu leisten. Nach Aussage des Geschäftsführers von ScanFarm bedeuteten die Entschädigungszahlungen an die einzelnen Landnutzer*innen aus Sicht des Unternehmens eine »Doppelzahlung« (Übersetzung der Autorin), da sie zusätzlich zum drink money und zur jährlichen Pachtzahlung anfielen ${ }^{41}$.

Das Gespräch unter Anwesenheit der Lands Commission und der EPA im Rahmen der Registrierung des Pachtvertrags verdeutlicht die Reichweite versteckter Macht. Bestimmte Optionen standen in diesem Gespräch von vornherein gar nicht zur Diskussion und Entscheidung. Wie auch bei der Landvergabe an BioFuel Africa sah die Agenda für das Gespräch nicht vor, dass sich Landnutzer*innen gegen die Landvergabe aussprechen könnten, sondern nur, dass sie sich mit dem Unternehmen auf Bedingungen einigten, unter denen sie ihr Land an ScanFarm verpachten würden.

Die Vorsitzenden von BioFuel Africa bewirkten durch die frühzeitige Involvierung einiger sub-chiefs und Landnutzer*innen im Verhältnis zu ScanFarm

\footnotetext{
40 Landnutzerin aus Nsonyameye, Interview am 25.4.2016; Landnutzerin aus Nsonyameye, Interview am 26.4.2016; Landnutzerin aus Nsonyameye, Interview am 27.4.2016.

41 Geschäftsführer ScanFarm, Interview am 4.4.2016.
} 
zunächst eine größere Transparenz. Anders als im Fall von ScanFarm war beispielsweise die Höhe des drink money, die das Unternehmen für die Bestätigung des Pachtvertrags durch den Regent of Dagbon, den Vertreter des Ya Naa, zahlte, öffentlich bekannt. Dennoch beschränkte das Handeln des paramount chiefs als zentralem Ansprechpartner und Entscheidungsträger im Rahmen der Landvergabe an BioFuel Africa den Zugang unterschiedlicher Landnutzer*innen zu Land. Unabhängig davon, welches Land von der Landvergabe betroffen war, teilte der Tijo Naa das drink money mit den sub-chiefs aus dem Gebiet, für das er zuständig war. Er beteiligte jedoch nicht alle, auf deren Zugang zu Land sich das Vorhaben auswirkte, sondern schloss migrantische Landnutzer*innen davon aus ${ }^{42}$. Einige von ihnen lebten zu diesem Zeitpunkt schon seit mehreren Jahrzehnten in der Gegend ${ }^{43}$. In Gruppendiskussionen und Interviews äußerten diese ihren Unmut darüber und berichteten über die Einschränkungen und negativen Auswirkungen, die das Vorhaben auf ihren Zugang zu Land hatte. Trotz der Wahrnehmung dieser Missstände leisteten sie jedoch keinen Widerstand, um sich für ihre Interessen einzusetzen. Ihren fehlenden Widerstand begründeten sie mit dem Argument, sich dem Willen des paramount chiefs und der sub-chiefs fügen zu müssen, da sie ihnen jeweils Zugang zu ihrem Land ermöglichten ${ }^{44}$. Um weiterhin auf dem Land leben zu können, sei es unabdingbar, sie als Entscheidungsträger anzuerkennen und sich ihnen gegenüber als loyal zu erweisen ${ }^{45}$. Zur Zeit der Datenerhebung hatte BioFuel Africa das Land bereits verlassen. Trotz aller negativen Auswirkungen, die das Vorhaben auf ihren Zugang zu Land gehabt hatte, hofften die Landnutzer*innen auf weitere Investoren, die »Entwicklung« in ihr Dorf bringen würden ${ }^{46}$. Damit übernahmen sie das Argument des paramount chief, das er für das Vorhaben von BioFuel Africa anführte. Obwohl die Aussagen der Landnutzer*innen zu BioFuel Africa ein deutlicher Hinweis dafür sind, dass die Umsetzung des Vorhabens im Widerspruch zu ihren Interessen stand,

42 Landnutzer und settler-chief aus Parachanayilli, Gruppendiskussion am 13.5.2016; Landnutzer*innen und sub-chief von Tuya, Gruppendiskussion am 14.5.2016; Landnutzer aus Changolinaaya, Interview am 12.5.2016.

43 Landnutzer und settler-chief aus Parachanayilli, Gruppendiskussion am 13.5.2016; Landnutzer aus Changolinaaya, Interview am 12.5.2016.

44 Landnutzer und settler-chief aus Parachanayilli, Gruppendiskussion am 13.5.2016; Landnutzer aus Changolinaaya, Interview am 12.5.2016.

45 Landnutzer aus Changolinaaya, Interview am 12.5.2016.

46 Landnutzer*innen und sub-chief von Tuya, Gruppendiskussion am 14.5.2016; Landnutzer*innen und sub-chief von Jashei, Gruppendiskussion am 15.5.2016. 
glaubten sie daran, dass ihre Situation beim nächsten Mal anders sein könnte $e^{47}$. Die Zukunftsvisionen, die sie im Gespräch entwarfen, standen jedoch im Widerspruch zu agrarindustriellen Vorhaben ${ }^{48}$. Ihr Wunsch war es, weiterhin in den Dörfern zu leben und Landwirtschaft zu betreiben, aber wohlhabender zu sein, um Zugang zu Wasser zu haben, ihren Kindern Bildung zu ermöglichen und eine gute medizinische Versorgung vor Ort zu haben.

Nachdem der Regent of Dagbon, die Landvergabe an BioFuel Africa bestätigt hatte, orientierte sich das Unternehmen an dem von staatlicher Seite aus vorgesehenen Ablauf für die Vergabe von Land. Die Vorsitzenden des Unternehmens beteiligten die entsprechenden Behörden wie die Lands Commission und die EPA und bemühten sich um die Registrierung des Pachtvertrags und die dafür notwendige Umweltgenehmigung. Im Rahmen der damit einhergehenden Umwelt- und Sozialverträglichkeitsprüfung gab das Unternehmen eine Studie durch ein unabhängiges Berater*innenteam in Auftrag (Bruce 2008; SGS Environment 2009). Ebenso wie ScanFarm begann jedoch auch BioFuel Africa bereits mit der Bearbeitung des Lands, bevor es über die entsprechenden staatlichen Genehmigungen verfügte. Auch versäumte das Unternehmen, die Landnutzer*innen über seine Anwesenheit zu informieren oder geplante Veränderungen auf dem Land im Vorfeld mit ihnen zu abzuklären ${ }^{49}$. Auf dem Treffen im Tamale Cultural Center hatten sich alle Beteiligten darauf geeinigt, dass das Unternehmen bei seinen Arbeiten auf dem Land die traditionelle Nutzung berücksichtigen solle. Dennoch führte BioFuel Africa seine Arbeiten durch, ohne noch einmal mit den Landnutzer*innen Rücksprache $\mathrm{zu}$ halten ${ }^{50}$. $\mathrm{Zu}$ diesem Ergebnis kam auch die durch das Unternehmen in Auftrag gegebene Studie zu den Auswirkungen des Jatropha-Anbaus auf die betroffenen Dörfer (Bruce 2008). Darüber hinaus hatten der paramount chief

47 Landnutzer und settler-chief aus Parachanayilli, Gruppendiskussion am 13.5.2016; Landnutzer*innen und sub-chief von Tuya, Gruppendiskussion am 14.5.2016.

48 Landnutzer*innen und settler-chief aus Kpachaa, Gruppendiskussion am 11.5.2016; Landnutzer und settler-chief aus Parachanayilli, Gruppendiskussion am 13.5.2016.

49 Landnutzer und settler-chief aus Parachanayilli, Gruppendiskussion am 13.5.2016; Landnutzer*innen und sub-chief von Tuya, Gruppendiskussion am 14.5.2016; Landnutzer*innen und sub-chief von Jashei, Gruppendiskussion am 15.5.2016.

50 Landnutzer und settler-chief aus Parachanayilli, Gruppendiskussion am 13.5.2016; Landnutzer*innen und sub-chief von Tuya, Gruppendiskussion am 14.5.2016; Landnutzer*innen und sub-chief von Jashei, Gruppendiskussion am 15.5.2016; Landnutzer aus Changolinaaya, Interview am 12.5.2016. 
oder der entsprechende sub-chief das Unternehmen zuvor nicht über die Anwesenheit von Landnutzer*innen auf dem Land informiert, wie im Fall der settler community Changolinaaya ${ }^{51}$. Die Kontrolle von Informationen ist hier als Ausübung versteckter Macht zu verstehen, da sie negative Auswirkungen auf den Zugang zu Land hatte. Da BioFuel Africa nicht über die auf dem gepachteten Land lebenden settler communities informiert war, bezog die Studie, die das Unternehmen für die Umweltverträglichkeitsprüfung in Auftrag gab, die Dörfer Changolinaaya und Parachanayilli ebenfalls nicht mit in ihre Untersuchung ein. Entsprechend erhob sie auch nicht die negativen Auswirkungen, die das Vorhaben von BioFuel Africa zu diesem Zeitpunkt bereits auf diese Dörfer hatte, sowie die Erwartungen der Landnutzer*innen an das Projekt. Die durch die Landnutzer*innen erlebten negativen Auswirkungen bildeten jedoch die Grundlage, auf der die Berater*innen dem Unternehmen mögliche Ansätze für eine Lösung oder für den Umgang damit unterbreiteten. Durch die Entscheidungen des paramount chiefs, einigen Landnutzer*innen Informationen vorzuenthalten und auch das Unternehmen nicht über alle auf dem Land befindlichen Dörfer in Kenntnis zu setzen, verschlechterte sich der $\mathrm{Zu}$ gang zu Land insbesondere für Landnutzer*innen mit schwachen Rechten und ohne persönliche Beziehungen zum paramount chief.

Obwohl in beiden Fällen die paramount chiefs offensichtlich gegen den Willen der Landnutzer*innen handelten, lehnten sich die sub-chiefs nicht gegen ihre Entscheidungen auf, obwohl es in ihrer Verantwortung liegt, sich für die Interessen ihrer Gemeinschaft einzusetzen. Aufgrund ihrer Abhängigkeit von dem paramount chief war ihre Loyalität ihm gegenüber stärker als ihre Verantwortung gegenüber der Gemeinschaft. Eine mögliche Erklärung dafür ist, dass die Position eines sub-chiefs im Wesentlichen von seiner Beziehung zum paramount chief abhängt, da dieser ihn ernennt. In den Interviews mit den sub-chiefs von Dukusen und Nsonyameye äußerten beide ihren Unmut bezüglich des Ablaufs der Landvergabe an ScanFarm. Sie betonten, dass Land laut Gewohnheitsrecht nicht ohne die Konsultierung der jeweiligen Landnutzer*innen vergeben werden solle ${ }^{52}$. Gleichzeitig erklärten sie jedoch, dass ihre Position es ihnen nicht erlaube, Entscheidungen des paramount chiefs infrage zu stellen. Er sei der Landeigentümer (owner of the land) und sie nur seine

$51 \quad$ Landnutzer aus Changolinaaya, Interview am 12.5.2016.

52 Sub-chief von Dukusen, Interview am 19.4.2016; sub-chief von Nsonyameye, Interview am 20.4.2016. 
Verwalter (care-taker). Daher müssten sie sich seinem Willen beugen ${ }^{53}$ Gleiches bestätigen die Aussagen der sub-chiefs von Jashei und Tuya bezüglich der Landvergabe an BioFuel Africa. Sie berichteten, sie hätten ihr Missfallen gegenüber dem Tijo Naa geäußert, könnten an seiner Entscheidung jedoch nichts ändern ${ }^{54}$. Des Weiteren verweisen die Erkenntnisse aus der empirischen Forschung darauf, dass eine gute Beziehung zum paramount chief ebenfalls bedeutsam für bestimmte Privilegien wie die Teilhabe an Projekten in Form von Arbeitsplätzen oder finanziellen Zuwendungen ist ${ }^{55}$. Der Ablauf der Landvergabe an ScanFarm illustriert dies besonders gut. In der Regel führen im Fall von Landvergaben an Investoren für kommerzielle oder Entwicklungszwecke die sub-chiefs eine Landbegehung mit den Investoren durch. Sie sind diejenigen, die sich am besten in der Gegend auskennen, wissen, wer welches Land nutzt und wo die Grenzen des durch sie verwalteten Lands verlaufen ${ }^{56}$. In der alltäglichen Verwaltung des Lands üben sie uneingeschränkte Kontrolle über den Zugang zu stool land aus. Im Rahmen der Landvergabe an ScanFarm betraute der paramount chief jedoch nicht den amtierenden sub-chief mit der Aufgabe, den Investoren das Land zu zeigen, sondern den ehemaligen subchief von Dukusen ${ }^{57}$. Dieser war 2001 aufgrund von illoyalem Verhalten gegenüber seiner Gemeinschaft des Amtes enthoben worden. Nach seiner Amtsenthebung schuf der paramount chief jedoch eigens für ihn den Posten des Informanten im ATC. Mit diesem Posten übertrug der paramount chief ihm die Verantwortung dafür, ihn über aktuelle Entwicklungen in der Gemeinschaft zu unterrichten. Auf das Drängen seiner Gemeinschaft hin, der gegenüber er sich als illoyal erwiesen hatte, wurde er seiner Position als sub-chief enthoben. Die persönliche Beziehung zum paramount chief, dem gegenüber er sich stets loyal verhalten hatte, ermöglichte es ihm jedoch trotz seiner Amtsenthebung

53 Sub-chief von Dukusen, Interview am 19.4.2016; sub-chief von Nsonyameye, Interview am 20.4.2016.

54 Landnutzer*innen und sub-chief von Tuya, Gruppendiskussion am 14.5.2016; Landnutzer*innen und sub-chief von Jashei, Gruppendiskussion am 15.5.2016.

55 Leiter des Department of Agriculture des Asante Akyem North District, Interview am 22.4.2016.

56 Registerführer des Agogo Traditional Council und Leiter des Agogo Customary Land Secretariat, Interview am 20.4.2016.

57 Landnutzeræinnen aus Nsonyameye, Baama \& Dukusen, Gruppendiskussion am 27.4.2016; sub-chief von Baama, Interview am 20.04.2016. 
Teil des ATC zu bleiben ${ }^{58}$. Darüber hinaus wird durch dieses Vorgehen ein weiteres Mal die versteckte Macht des paramount chiefs deutlich. Die Umsetzung der Entscheidung, den zuständigen sub-chief nicht in die Vergabe des Lands einzubeziehen, sondern diese Aufgabe seinem Informanten zu übertragen, kann als Einflussnahme auf gewohnheitsrechtliche Normen und Regeln verstanden werden. Versteckte Macht über Landnutzer*innen und subchiefs auszuüben, bedeutet auch in diesem Fall wieder, dass der paramount chief entscheiden konnte, an wen er Informationen weitergibt und an wen nicht, um so den Zugang der Landnutzer*innen zu Land zu beschränken, ohne dass dies für ihn mit späteren Sanktionen verbunden war.

Anders als die sichtbare und versteckte Macht lässt sich die unsichtbare Macht der paramount chiefs über die sub-chiefs und Landnutzer*innen nicht über ihr Handeln erfassen, sondern nur über die Reaktionen ihrer Gegenspieler*innen auf ihr Handeln. Die Analyse der Konflikte um ScanFarm und BioFuel Africa deutet jedoch darauf hin, dass diese Form der Macht eine grundlegende in traditionellen Herrschaftssystemen ist. Die Fähigkeit traditioneller Autoritäten unsichtbare Macht auszuüben - oder die Einstellungen, Ansichten, Wünsche und Ziele anderer zu beeinflussen - beruht auf ihrer Anerkennung durch die Mitglieder ihrer Gemeinschaft. Sie erkennen traditionelle Autoritäten an, da dies die Voraussetzung für ein Leben in der Gemeinschaft ist (Berry 2008). Die Anerkennung traditioneller Autoritäten durch die Gemeinschaft bildet im Umkehrschluss die Grundlage für die Legitimität ihres Handelns. Folglich stellen die Mitglieder der Gemeinschaft das Handeln traditioneller Autoritäten selten infrage, sondern akzeptieren ihre Entscheidungen. Eine Möglichkeit um die Ausübung unsichtbarer Macht dennoch erkennen zu können, ist die Ermittlung der ethischen Interessen der sub-chiefs und Landnutzer*innen unter sich verändernden Zugangsbedingungen. Als ethische Interessen fasse ich unter Rückgriff auf meinen theoretischen Analyserahmen eine angemessene Entlohnung, einen angemessenen Lebensstandard - der sich über angemessene Nahrung, Kleidung und Wohnung ermitteln lässt - sowie den Zugang zu Bildung. Verschlechtern sich die Lebensbedingungen bezüglich dieser Interessen aufgrund des veränderten Zugangs zu Land, ist das Handeln, das diese Veränderung nach sich zog als widersprüchlich zu bewerten. Wenn Akteure - in diesem Fall sub-chiefs und Landnutzer*innen - Entscheidungen, die ihren ethischen Interessen entgegenstehen, widerspruchs20.04.2016. 
los akzeptieren oder sogar ihre vermeintlichen Vorteile aufzählen, ist dies ein Hinweis auf die Ausübung unsichtbarer Macht. Mangelnder Widerstand kann darauf zurückzuführen sein, dass Akteure bestimmte Handlungsoptionen aufgrund von Sozialisation oder gesellschaftlichen Normen und Regeln nicht als solche erkennen. Eine weitere Möglichkeit um Widersprüche bezüglich der ethischen Interessen von Akteuren zu ermitteln, besteht darin ihr kontrafaktisches Handeln zu bestimmen. Kontrafaktisches Handeln bezeichnet Entscheidungen, die Akteure treffen oder bevorzugen würden, wenn sie frei von gesellschaftlichen, strukturellen und wirtschaftlichen Zwängen entscheiden könnten (Lukes 2005: 38). Empirisch lässt es sich über die Entwicklung von Zukunftsvisionen erfassen.

In der Landvergabe an ScanFarm kommt die unsichtbare Macht des paramount chiefs darüber zum Ausdruck, dass die meisten der betroffenen Landnutzer*innen davon absahen, ihr Land von dem Unternehmen zurückzufordern, nachdem der paramount chief ihnen während eines Treffens im Palast des paramount chiefs am 25. Januar 2010 (FoodSPAN 2011) dazu geraten hatte, dem Unternehmen die Nutzung des Lands zu gestatten. Er argumentierte das Projekt bringe Entwicklung, Beschäftigungsmöglichkeiten sowie Infrastruktur $^{59}$, und da es bereits mit der Bearbeitung des Lands begonnen habe, sollten die Landnutzer*innen lieber Entschädigungszahlungen aushandeln, anstatt ihr Land zurückzufordern ${ }^{60}$. Die Untätigkeit dieser Landnutzer*innen bezüglich des Verlusts ihres Zugangs zu Land bedeutet jedoch nicht zwangsläufig, dass sie an die Vorteile des Vorhabens für sie selbst glaubten. In Interviews berichteten Landnutzer*innen stattdessen, dass sie keine Möglichkeit sahen, die Entscheidung des paramount chiefs in Frage zu stellen ${ }^{61}$. Aufgrund des tief verinnerlichten Respekts gegenüber traditionellen Autoritäten stellten die meisten Landnutzer*innen die Meinung des paramount chiefs nicht infrage. Daran änderte sich auch später nichts, als sich herausstellte, dass die

59 Landnutzer*innen aus Nsonyameye, Baama \& Dukusen, Gruppendiskussion am 27.4.2016; Leiter des Department of Agriculture des Asante Akyem North District, Interview am 22.4.2016; Sub-chief von Dukusen, Interview am 19.4.2016; sub-chief von Nsonyameye, Interview am 20.4.2016.

60 Landnutzer*innen aus Nsonyameye, Baama \& Dukusen, Gruppendiskussion am 27.4.2016.

61 Landnutzerin aus Nsonyameye, Interview am 25.4.2016; Landnutzer aus Nsonyameye, Interview am 25.4.2016; Landnutzerin aus Nsonyameye, Interview am 27.4.2016. 
Entschädigungszahlungen sehr viel geringer waren, als sie gehofft hatten ${ }^{62}$ und ihr Einkommen aus dem Land, im Gegensatz zu vorher, nicht mehr ausreichte, um das Schulgeld für die Kinder zu bezahlen ${ }^{63}$ oder ausreichend Essen für die Familie zu kaufen ${ }^{64}$. Die unsichtbare Macht des paramount chiefs äußerte sich also darin, dass er Entscheidungen, die sich letztendlich negativ auf die ethischen Interessen der Landnutzer*innen auswirkten, durchsetzen konnte, ohne dass diese dagegen Widerstand leisteten.

In der Landvergabe an BioFuel Africa äußerte sich die unsichtbare Macht des paramount chiefs auf gleiche Weise. Auch hier leisteten die in der Gegend lebenden sub-chiefs und Landnutzer*innen größtenteils keinen Widerstand gegen die Landvergabe und den Verlust der Zugangskontrolle, sondern akzeptierten die Entscheidung des paramount chiefs. Ihren mangelnden Widerstand erklärten Landnutzer*innen und sub-chiefs damit, dass der paramount chief ihnen versichert habe, BioFuel Africa würde Arbeitsplätze schaffen, ein Krankenhaus bauen und den Zugang zu Wasser sowie die Gesamtsituation der in der Gegend lebenden Menschen verbessern ${ }^{65}$. Über die Analyse wird jedoch deutlich, dass die Landvergabe an BioFuel Africa in deutlichem Widerspruch zu ihren ethischen Interessen steht. Landnutzer*innen aus allen betroffenen Dörfern berichteten von negativen Folgen, welche die Veränderung des Zugangs zu Land mit sich brachte, statt von Verbesserungen. Zu den negativen Folgen zählten insbesondere der Verlust von Einnahmen durch das Fällen von Nutzbäumen und der Verlust von Ackerflächen ${ }^{66}$. Ihr fehlender Widerstand lässt sich darüber erklären, dass sie akzeptierten, sich aufgrund

62 Landnutzerin aus Nsonyameye, Interview am 24.4.2016; Landnutzerin aus Nsonyameye, Interview am 25.4.2016; Landnutzerin aus Nsonyameye, Interview am 27.4.2016; Landnutzerin aus Nsonyameye, Interview am 27.4.2016; sub-chief von Dukusen, Interview am 19.4.2016.

63 Landnutzer*innen aus Nsonyameye, Baama \& Dukusen, Gruppendiskussion am 27.4.2016; Landnutzerin aus Nsonyameye, Interview am 27.4.2016.

64 Landnutzer*innen aus Nsonyameye, Baama \& Dukusen, Gruppendiskussion am 27.4.2016; Landnutzerin aus Nsonyameye, Interview am 24.4.2016; Landnutzerin aus Nsonyameye, Interview am 26.4.2016; Landnutzerin aus Dukusen, Interview am 16.3.2017.

65 Landnutzer*innen aus Jimle und Gumani, Gruppendiskussion am 10.5.2016; Landnutzer*innen und settler-chief aus Kpachaa, Gruppendiskussion am 11.5.2016; Landnutzer*innen und sub-chief von Tuya, Gruppendiskussion am 14.5.2016; Landnutzer*innen und sub-chief von Jashei, Gruppendiskussion am 15.5.2016.

66 Landnutzer*innen und settler-chief aus Kpachaa, Gruppendiskussion am 11.5.2016; Landnutzer und settler-chief aus Parachanayilli, Gruppendiskussion am 13.5.2016; 
gesellschaftlicher Normen nicht gegen die Entscheidung des paramount chiefs stellen zu können ${ }^{67}$. Sie waren mit dem Handeln des paramount chiefs nicht einverstanden, erkannten jedoch an, kein Mitspracherecht zu haben ${ }^{68}$. Die migrantischen Landnutzer*innen erklärten ihren fehlenden Widerstand darüber, dass ihnen das Land nicht gehöre und sie dementsprechend auch nicht darüber entscheiden könnten ${ }^{69}$. Aus Angst den paramount chief gegen sich aufzubringen und gegebenenfalls gezwungen $\mathrm{zu}$ sein, die Gegend verlassen $\mathrm{zu}$ müssen, könnten sie es sich nicht erlauben seine Entscheidungen zu beurteilen oder in Frage zu stellen ${ }^{70}$.

Die unsichtbare Macht traditioneller Autoritäten ist darüber hinaus in Konsultationsverfahren oder auf Versammlungen zu beobachten. Wenn traditionelle Autoritäten bei diesen Treffen anwesend sind, äußern Mitglieder der Gemeinschaft in der Regel keine Meinung, die der Meinung des chiefs widerspricht, noch üben sie Kritik an seinen Entscheidungen ${ }^{71}$. Das gilt ebenso für den paramount chief wie auch für die sub-chiefs. Derartige Äußerungen in Anwesenheit traditioneller Autoritäten gelten als Mangel an Respekt. In Konsultationsverfahren trauen sich viele Landnutzer*innen in Anwesenheit traditioneller Autoritäten entsprechend nicht, Forderungen $\mathrm{zu}$ formulieren, die den Meinungen traditioneller Autoritäten widersprechen ${ }^{72}$.

Aufgrund der sozialen Differenzierung von Landnutzer*innen, den unterschiedlichen Mechanismen, über die sie Zugang zu Land erlangen können und ihres Verhältnisses zu traditionellen Autoritäten, konnten die paramount chiefs jedoch nicht über alle Landnutzer*innen gleichermaßen unsichtbare Macht ausüben. Die unsichtbare Macht traditioneller Autoritäten beruht auf der Wahrnehmung ihres Handelns als legitim sowie auf fehlenden

Landnutzer*innen und sub-chief von Tuya, Gruppendiskussion am 14.5.2016; Landnutzer"innen und sub-chief von Jashei, Gruppendiskussion am 15.5.2016.

67 Landnutzer aus Changolinaaya, Interview am 12.5.2016.

68 Landnutzer*innen und sub-chief von Tuya, Gruppendiskussion am 14.5.2016; Landnutzer"innen und sub-chief von Jashei, Gruppendiskussion am 15.5.2016.

69 Landnutzer*innen und settler-chief aus Kpachaa, Gruppendiskussion am 11.5.2016; Landnutzer und settler-chief aus Parachanayilli, Gruppendiskussion am 13.5.2016.

70 Landnutzer und settler-chief aus Parachanayilli, Gruppendiskussion am 13.5.2016.

71 Leiter des Department of Agriculture Asante Akyem North District, persönliches Gespräch am 25.04.2016; eigene Beobachtung; Research Officer des Regional House of Chiefs der Northern Region, Interview am 8.3.2017.

72 Mitarbeiterin der Civil Society Coalition on Land, Interview am 22.3.2016. 
Handlungsoptionen, um ihre Entscheidung anzufechten. Im Kontext großflächiger Landvergaben äußert sich die Legitimität des Handelns traditioneller Autoritäten in ihrer Anerkennung als Landeigentümer. Landnutzer*innen, die Zugang zu Land über eine traditionelle Autorität erlangt haben, müssen dieser ihren Respekt und ihre Loyalität entgegenbringen, um auch in der $\mathrm{Zu}$ kunft ihren Zugang zu Land aufrechterhalten zu können (Amanor 2008; Berry 1989; Boone 2013). Dazu zählen auch das Respektieren der Entscheidungen traditioneller Autoritäten und ihre Anerkennung als Landeigentümer. Im Kontext der großflächigen Vergabe von Land setzt sich dieses Prinzip entlang der Hierarchie fort. Entsprechend erkennen die sub-chief das Handeln des paramount chiefs als legitim an, da er die Quelle ihrer Macht verkörpert ${ }^{73}$. Landnutzer*innen, deren Zugang zu Land sich über die erstmalige Bestellung des Lands oder die erste Niederlassung in einer Gegend ableitet, sind nicht auf den sub-chief angewiesen, um Landrechte zu erhalten. In der Gegend von ScanFarm bezeichnen diese Landnutzer*innen sich auch selbst als Landeigentümer*innen ${ }^{74}$. Da in Bezug auf den Zugang zu Land faktisch keine Verbindung zwischen diesen Landnutzer*innen und traditionellen Autoritäten existiert, stehen sie auch in keinem Abhängigkeitsverhältnis zu diesen. Die Analyse lässt darauf schließen, dass der paramount chief über diese Akteure keine unsichtbare Macht ausüben kann.

Landnutzer*innen aus Nsonyameye und Baamaa, die über die erste Niederlassung in der Gegend oder die erstmalige Bestellung des Lands permanente Rechte an Land geltend machten, waren es auch, die den stärksten Widerstand gegen die Landvergabe an ScanFarm leisteten und dem Konflikt zivilgesellschaftliche und mediale Aufmerksamkeit verschafften ${ }^{75}$. Sie gaben sich nicht mit dem Versprechen des paramount chiefs, das Projekt bringe Entwicklung, zufrieden. Auch akzeptierten sie nicht die geringen Entschädigungszahlungen, die ihnen ScanFarm für ihr Land bot. Stattdessen wandten sie sich

Landnutzerin aus Nsonyameye, Interview am 25.4.2016; Landnutzer aus Dukusen, Interview am 26.4.2016; Leiter des Department of Agriculture des Asante Akyem North District, Interview am 22.4.2016; sub-chief von Dukusen, Interview am 19.4.2016; subchief von Nsonyameye, Interview am 20.4.2016.

74 Landnutzer aus Nsonyameye, Interview am 28.4.2016; Landnutzerer aus Nsonyameye und Mitglieder der Agogoman mma kuo, Interview am 16.3.2017; Landnutzer aus Nsonyameye und Vorsitzender der Agogoman mma kuo, Interview am 16.3.2017. 16.3.2017; Landnutzer aus Nsonyameye und Vorsitzender der Agogoman mma kuo, Interview am 16.3.2017. 
offen gegen den Willen des paramount chiefs und forderten ihr Land zurück ${ }^{76}$. Mit diesem Ziel verfassten sie zunächst Briefe an die District Assembly, das Unternehmen und die Lands Commission, um diese zu informieren, dass sie nicht bereit seien, ScanFarm ihr Land zur Verfügung zu stellen ${ }^{77}$. Da ScanFarm mit Verweis auf den Pachtvertrag zwischen dem Unternehmen und dem ATC weiterhin Anspruch auf das Land erhob und seine Arbeiten darauf fortsetzte ${ }^{78}$, engagierten einige dieser Landnutzer*innen einen Anwalt, um ihr Land auf dem Rechtsweg zurückzufordern ${ }^{79}$. Das Gerichtsverfahren dauerte zum Zeitpunkt des letzten Feldforschungsaufenthalts im Februar 2017 noch an ${ }^{80}$.

Im Konflikt um die Landvergabe an BioFuel Africa ging der größte Widerstand von einer anderen Landnutzer*innengruppe aus. Hier mobilisierten Landwirt*innen, die aus Tamale pendelten (commuter farmers) gegen das Vorhaben. Sie lebten in der Regionalhauptstadt und kamen täglich in die Projektgegend, um Land zu bewirtschaften. Zugang zu diesem Land hatten sie über einen der sub-chiefs aus der Gegend erlangt. Im Vergleich zu den in der Projektgegend lebenden Landnutzer*innen waren sie relativ wohlhabend, was sich darin äußerte, dass sie Traktoren für die mechanisierte Landwirtschaft besaßen und Landnutzer*innen aus der Gegend als Lohnarbeiter*innen beschäftigten (Tsikata/Yaro 2011: 21). Ihre Zugangsrechte zu Land sind vergleichbar mit denen von migrantischen Landnutzer*innen, die in der Gegend lebten. Sie bestehen so lange, wie sie das Land bestellen. Wird die Fläche für ein Vorhaben, das dem Wohl der Gemeinschaft dient, benötigt oder erheben indigene Landnutzer*innen Anspruch darauf, können sie die Nutzungsrechte jederzeit - in der Regel nach der Ernte - und ohne Entschädigung verlieren ${ }^{81}$.

76 Landnutzer aus Nsonyameye, Interview am 28.4.2016; Landnutzerer aus Nsonyameye und Mitglieder der Agogoman mma kuo, Interview am 16.3.2017; Landnutzer aus Nsonyameye und Vorsitzender der Agogoman mma kuo, Interview am 16.3.2017.

77 Brief der Landnutzerinnen von Baama an den Vorsitzenden der Asante Akyem North Distric Assembly, 1.2.2011; Brief der Landnutzerinnen von Baama an den Vorsitzenden der Asante Akyem North Distric Assembly, 16.2.2011. Landnutzer aus Nsonyameye, Interview am 28.4.2016; Landnutzerer aus Nsonyameye und Mitglieder der Agogoman mma kuo, Interview am 16.3.2017; Landnutzer aus Nsonyameye und Vorsitzender der Agogoman mma kuo, Interview am 16.3.2017.

79 Brief des Anwalts der Landnutzer*innen von Baama an den Geschäftsführer vonScanFarm, 11.7.2012.

80 Landnutzer aus Nsonyameye und Vorsitzender der Agogoman mma kuo, Interview am 16.3.2017.

81 Paramount-chief und elders von Tijo, Gruppendiskussion am 8.5.2016. 
Dieser Fall trat mit der Landvergabe an BioFuel Africa ein, so dass sie ihre Felder an das Unternehmen verloren und die Gegend verlassen mussten ${ }^{82}$. Der Unterschied dieser Landwirt*innen zu den in der Gegend lebenden migrantischen Landnutzer*innen ist jedoch, dass sie außer ihrem Land nichts zu verlieren hatten. Sie gehörten nicht zu der lokalen Gemeinschaft und waren daher, abgesehen von ihrem Zugang zu Land, nicht auf das Wohlwollen traditioneller Autoritäten angewiesen ${ }^{83}$. Nachdem sie ihr Land verloren hatten, machten sie unter anderem Action Aid Ghana auf den Fall aufmerksam und informierten in Radiosendungen über die Landvergabe an BioFuel Africa, um zivilgesellschaftliche und mediale Aufmerksamkeit zu erlangen. Das war jedoch nicht im Sinne anderer migrantischer Landnutzer*innen, die Angst hatten, dafür verantwortlich gemacht zu werden und ihr Land ebenfalls verlassen zu müssen ${ }^{84}$.

Diejenigen Landnutzer*innen, die Widerstand gegen die Landvergabe leisteten und die Entscheidungen der traditionellen Autoritäten herausforderten, waren folglich diejenigen, die sie nicht als Landeigentümer anerkannten oder die außer des Zugangs zu Land nichts zu verlieren hatten. Ihnen gegenüber hatten die paramount chiefs keine unsichtbare Macht, da sie aufgrund ihrer Sozialisation, ihres Bildungsstands oder ihrer Klassenpositionen Handlungsoptionen erkannten, die andere Landnutzer*innen nicht hatten. Unsichtbare Macht ist wirksam gegenüber Landnutzer*innen mit unbeständigem Zugang zu Land und die diesen unbeständigen Zugang auch aufgrund ihrer Sozialisation anerkennen. Sie sind diejenigen, die in den Interviews mehrmals betonten, »keinen Widerstand leisten $\mathrm{zu}$ können « ${ }^{85}$, »ihre Stimme nicht erheben zu können ${ }^{86}$, »nichts zu sagen zu haben ${ }^{87}$ oder "nichts gegen die Entscheidung des paramount chiefs tun zu können ${ }^{88}$.

\footnotetext{
82 Landnutzer*innen und sub-chief von Tuya, Gruppendiskussion am 14.5.2016.

83 Landnutzer aus Changolinaaya, Interview am 12.5.2016.

84 Landnutzer aus Changolinaaya, Interview am 12.5.2016.

85 Landnutzer und settler-chief aus Parachanayilli, Gruppendiskussion am 13.5.2016.

86 Landnutzer*innen und sub-chief von Jashei, Gruppendiskussion am 15.5.2016.

87 Landnutzer und settler-chief aus Parachanayilli, Gruppendiskussion am 13.5.2016.

88 Landnutzer*innen und sub-chief von Jashei, Gruppendiskussion am 15.5.2016.
} 


\subsection{Konflikte um Zugang zu Land im Spannungsfeld der drei Machtformen}

Die Analyse der beiden Konflikte entlang der drei Machtformen ermöglicht es nachzuvollziehen, warum traditionelle Autoritäten so handeln können, wie sie handeln und inwiefern ihr Handeln die Entstehung und den Verlauf von Konflikten beeinflusst. Die Machtanalyse trägt darüber hinaus dazu bei, die (fehlenden) Reaktionen von Gegenspieler"innen im Konflikt zu verstehen und liefert Erklärungsansätze für die Frage, warum sich Konflikte zu bestimmten Zeitpunkten und entlang der Verhandlung bestimmter Themen manifestieren oder latent verlaufen. Außerdem wird über die Analyse deutlich, warum Konflikte auch als solche verstanden werden müssen, wenn widersprüchliche Interessen nicht in Handeln resultieren und warum die Interessen der einzelnen Konfliktakteure nur teilweise verhandelt werden. Indem wir die sichtbare, versteckte und unsichtbare Macht nicht als getrennt voneinander betrachten, sondern insbesondere auch ihre Wechselwirkungen in den Blick nehmen, gelingt es komplexe Zusammenhänge, die sich auf den Verlauf von Konflikten auswirken, zu erfassen.

Typische Arten der Machtausübung, derer sich beide paramount chiefs bedienten, waren die Verfügungsgewalt über Informationen und die Neuinterpretation institutioneller Verfahrensweisen. Diese Handlungsstrategien gingen mit dem Ausschluss anderer, für die Landvergabe zentraler Akteure wie sub-chiefs, Landnutzer*innen und Mitarbeiter*innen von Behörden einher, der dazu führte, dass zunächst kein Konflikt entstand. Denn Gegenspieler*innen konnten nicht erkennen, dass die paramount chiefs entgegen ihres Willens handelten. Im Fall von ScanFarm wurde der Konflikt erst manifest, als die Landnutzer*innen feststellten, dass jemand ohne ihre Erlaubnis ihr Land bearbeitete. Später erhielten sie die Information, dass der paramount chief ihr Land an das Unternehmen vergeben hatte und damit bereits zuvor gegen ihre Interessen gehandelt hatte. Ihren Ausschluss von der Verhandlung der Landvergabe, die fehlende Konsultierung und die damit einhergehende Intransparenz gaben viele Landnutzer*innen als Ursprung des Konflikts an ${ }^{89}$. Im Gegensatz dazu war BioFuel Africa im Kontext der

89 Landnutzerin aus Nsonyameye, Interview am 24.4.2016; Landnutzerin aus Nsonyameye, Interview am 24.4.2016; Landnutzer aus Nsonyameye, Interview am 25.4.2016; Landnutzer aus Dukusen, Interview am 26.4.2016; Landnutzerin aus Nsonyameye, Interview am 26.4.2016; Landnutzerer aus Nsonyameye und Mitglieder der Agogoman 
Landvergabe bestrebt, alle betroffenen Landnutzer*innen im Rahmen einer Versammlung über ihr Vorhaben zu informieren. Dies änderte jedoch nichts an dem Ausschluss der Landnutzer*innen von Entscheidungen. Migrantische Landnutzer*innen schloss der paramount chief unmittelbar aus, indem er sie nicht einlud und das Unternehmen nicht über ihre Anwesenheit informierte. Die anwesenden indigenen Landnutzer*innen erhielten auf dem Treffen zwar grundlegende Informationen über das Vorhaben des Unternehmens, hatten jedoch kein Mitspracherecht und auch nicht die Möglichkeit Widerspruch zu erheben. Stattdessen legte der paramount chief ihnen die Vorteile des Vorhabens dar. Aufgrund der unsichtbaren Macht des paramount chiefs schenkten die Landnutzer*innen und sub-chiefs ihm Glauben. Die gleiche Strategie war auch die Antwort auf die Manifestierung des Konflikts um die Landvergabe an ScanFarm. Auch hier versuchte der paramount chief die subchiefs und Landnutzer"innen, die eigentlich gekommen waren, um ihr Land zurückzufordern, von dem Vorhaben des Unternehmens zu überzeugen, indem er die möglichen positiven Auswirkungen des Vorhabens aufzählte. Obwohl der Pachtvertrag über das Land bereits unterzeichnet war, zeigte er Verständnis für das Widerstreben der Landnutzer*innen und erkannte ihre Rechte an dem Land an, indem er ihnen riet, Entschädigungszahlungen für ihr Land auszuhandeln. Aufgrund unsichtbarer Macht akzeptierten die meisten Landnutzer*innen diese Möglichkeit als einzige Handlungsoption und leisteten keinen weiteren Widerstand. Das erklärt, warum sich der Konflikt um ScanFarm so stark an der Aushandlung von Entschädigungszahlungen manifestierte und kein breiter Widerstand gegen den paramount chief als Verantwortlichem für die Landvergabe entstand.

Die versteckte Macht der paramount chiefs - also ihre Einflussnahme auf institutionelle Verfahrensweisen oder deren Neuinterpretation sowie die Verfügungsgewalt über Informationen - wird durch ihre unsichtbare Macht gefestigt. Die Anerkennung der paramount chiefs durch sub-chiefs und Landnutzer*innen, die damit verbundene Legitimität ihres Handelns und der Mangel an Widerstand führten dazu, dass Gegner*innen, auf die sie unsichtbare Macht ausüben, ihre versteckte Macht nicht anfechten und ihr Handeln infrage stellen oder herausfordern. Die versteckte Macht der paramount chiefs

mma kuo, Interview am 16.3.2017; Landnutzerin aus Dukusen, Interview am 16.3.2017; Landnutzer aus Nsonyameye und Vorsitzender der Agogoman mma kuo, Interview am 16.3.2017. 
wird zudem durch unklare Zuständigkeiten gestützt. »Nicht-Handeln« entgegen der Interessen der Landnutzer*innen legitimierten die paramount chiefs in beiden Fällen damit, dass sie nicht zuständig gewesen seien ${ }^{90}$. Insbesondere den Vorwurf der mangelnden Informationen seitens der Landnutzer* innen wiesen beide mit dem Argument zurück, dafür seien nicht sie, sondern das Unternehmen, die sub-chiefs oder Behörden zuständig ${ }^{91}$.

In Bezug auf die Ausübung sichtbarer Macht - also die Durchsetzung der eigenen Interessen auch gegen Widerstreben - unterschied sich das Handeln der beiden paramount chiefs. Im Konflikt um die Landvergabe an BioFuel Africa handelte der Tijo Naa zu keinem Zeitpunkt offensichtlich gegen die Interessen der Landnutzer*innen und sub-chiefs. Wann sich immer diese an ihn wandten, um ihren Unmut mitzuteilen, hörte er sich ihre Beschwerden an, entschuldigte sich für mangelnde Informationen, gestand eigene Fehler ein und versicherte, alles werde sich zum Guten wenden ${ }^{92}$. Im Fall von ScanFarm beruht die Fähigkeit des paramount chiefs, sichtbare Macht auszuüben insbesondere auf seinem hohen Bildungsniveau. Als ausgebildeter Jurist kennt er sich gut mit dem Rechtssystem aus. Er verfügt über umfassende Kenntnisse bezüglich des Ablaufs von Landvergabeverfahren, weiß, welche Formalitäten er respektieren muss und welche er umgehen kann. Sichtbare Macht übte jedoch auch er nie offen gegenüber den Landnutzer*innen aus, sondern nur in Form eines Briefes an das Unternehmen, in dem er die Erlaubnis erteilte, auch gegen Widerstreben der Landnutzer*innen auf dem Land zu arbeiten. Die Ausübung sichtbarer Macht zählt also nicht zu den typischen Handlungsstrategien traditioneller Autoritäten. Die Unternehmen hingegen verfolgten ihre Ziele, nachdem sie Zugang zu dem Land über die paramount chiefs erhalten hatten, insbesondere über die Ausübung sichtbarer Macht. Sie setzten ihre Arbeiten trotz Widerspruch der Landnutzer*innen fort ${ }^{93}$, fällten Bäume,

90 Landnutzer*innen und sub-chief von Jashei, Gruppendiskussion am 15.5.2016; subchief von Nsonyameye, Interview am 20.4.2016; sub-chief von Baama, Interview am 20.04.2016.

91 Landnutzer*innen aus Jimle und Gumani, Gruppendiskussion am 10.5.2016; Landnutzer*innen und sub-chief von Jashei, Gruppendiskussion am 15.5.2016; sub-chief von Nsonyameye, Interview am 20.4.2016; sub-chief von Baama, Interview am 20.04.2016.

92 Landnutzer*innen und sub-chief von Tuya, Gruppendiskussion am 14.5.2016; Landnutzer*innen und sub-chief von Jashei, Gruppendiskussion am 15.5.2016.

93 Landnutzer aus Nsonyameye, Interview am 28.4.2016; Landnutzerer aus Nsonyameye und Mitglieder der Agogoman mma kuo, Interview am 16.3.2017; Landnutzerin aus Du- 
obwohl Landnutzer*innen sie baten, dies zu unterlassen ${ }^{94}$, und entschieden über die Höhe der Entschädigungszahlungen. Das funktionierte, da die paramount chiefs ihnen durch die Pachtverträge de facto die Kontrolle über den Zugang zu dem Land übertragen hatten.

Lukes Machtverständnis ermöglicht uns zu erkennen, auf welche Weise traditionelle Autoritäten Kontrolle über Land erlangen und ausüben können und welcher Strategien sie sich im Fall von Konflikten bedienen, um ihrer Verantwortung als Konfliktschlichter nachzukommen und gleichzeitig die eigenen Interessen durchzusetzen. Damit ergänzt es das theoretische Verständnis um Zugang zu Land, das Ribot und Peluso (2003) entwickelt haben, und hilft $\mathrm{zu}$ verstehen, wie Zugang zu Land in traditionellen Landverwaltungssystemen gesichert werden kann.

kusen, Interview am 16.3.2017; Landnutzer aus Nsonyameye und Vorsitzender der Agogoman mma kuo, Interview am 16.3.2017.

94 Landnutzerin aus Nsonyameye, Interview am 27.4.2016; Landnutzerin aus Dukusen, Interview am 16.3.2017: Landnutzer aus Nsonyameye und Vorsitzender der Agogoman mma kuo, Interview am 16.3.2017. 



\section{Fazit}

Ausgangspunkt meiner Forschung waren folgende Beobachtungen. Seit Beginn der 200oer Jahre nehmen Konflikte um Land $\mathrm{zu}$, insbesondere im $\mathrm{Zu}$ sammenhang mit land grabbing. In diesem Kontext verändern sich neben der Nutzung des Lands vor allem Kontrollverhältnisse hinsichtlich des Zugangs $\mathrm{zu}$ Land. In der wissenschaftlichen Literatur werden diese Konflikte daher auch als Konflikte um Zugang zu Land gefasst. Im globalen Vergleich ist Subsahara-Afrika besonders stark von land grabbing betroffen. Das gilt vor allem für gewohnheitsrechtlich oder traditionell verwaltetes Land, das in dieser Weltregion im Durchschnitt ca. 70 \% aller Flächen ausmacht, in der Hälfte aller afrikanischen Staaten sogar $80 \%$ oder mehr (Alden Wily 2017). Für die gewohnheitsrechtliche Verwaltung von Land sind in vielen Fällen traditionelle Autoritäten verantwortlich. Aktuelle Studien zu land grabbing in SubsaharaAfrika verweisen darauf, dass traditionelle Autoritäten ebenfalls eine zunehmend wichtige Rolle als Verhandlungspartner*innen in der Vergabe von Land an Investoren spielen (Ahmed et al. 2018; Boamah 2014a; Campion/Acheampong 2014; Capps 2018; Ndi/Batterbury 2017). Als solche sind sie zentrale Akteure in Konflikten um Zugang zu Land, die im Zusammenhang mit land grabbing auftreten. Die bestehende Literatur zu land grabbing und Konflikten gibt jedoch kaum Aufschluss über ihre Rolle als Akteure in diesen Konflikten.

Das ist verwunderlich da die Zuständigkeitsbereiche traditioneller Autoritäten, für die sie neben der Verwaltung und Vergabe von Land verantwortlich sind, Ansatzpunkte für die Analyse von Konflikten bieten. Laut Gewohnheitsrecht sind traditionelle Autoritäten die Repräsentant*innen ihrer Gemeinschaft. Als solche ist es ihre Aufgabe, die Interessen der Gemeinschaft nach außen zu vertreten und im Sinne der Gemeinschaft zu handeln. Darüber hinaus agieren sie als Mediator*innen in Konflikten, die innerhalb der Gemeinschaft auftreten, sowie in Konflikten zwischen Mitgliedern der Gemeinschaft und Außenstehenden. In Konflikten im Zusammenhang mit land 
grabbing kommen Widersprüche bezüglich des Handelns traditioneller Autoritäten zum Ausdruck. Wenn wir davon ausgehen, dass Konflikte entstehen, wenn mindestens zwei Akteure, die in Beziehung zueinander stehen, gegensätzliche Interessen, Ziele oder Bedürfnisse haben, sollten traditionelle Autoritäten durch ihr Handeln als Interessensvertreter*innen der Gemeinschaft innergemeinschaftlichen Konflikten vorbeugen. Treten solche Konflikte dennoch auf, lässt dies darauf schließen, dass traditionelle Autoritäten entgegen der gemeinschaftlichen Interessen und somit entgegen ihrer Zuständigkeit gehandelt haben. Wie lassen sich Konflikte um Zugang zu Land, in denen traditionelle Autoritäten zentrale Akteure sind, erklären? Inwiefern verändert sich traditionelle Herrschaft im Zusammenhang mit land grabbing? Wie verändert sich in diesem Zusammenhang Kontrolle über den Zugang zu Land? Welche Rolle spielen traditionelle Autoritäten für die Entstehung und den Verlauf von Konflikten, die im Zusammenhang mit der Vergabe von Land an Investoren auftreten? Diesen Fragen bin ich in meiner Forschung unter folgender übergeordneter Fragestellung nachgegangen: Welcher Zusammenhang besteht zwischen dem Handeln traditioneller Autoritäten und Konflikten um Zugang zu Land im Kontext von land grabbing?

Aus der Literatur zu land grabbing und Konflikten sowie zu traditionellen Landverwaltungssystemen habe ich drei Annahmen abgeleitet und meiner Untersuchung zugrunde gelegt. Diese Annahmen haben das Design sowie den theoretischen Rahmen meiner Forschung bestimmt. Die erste Annahme war, dass traditionelle Herrschaft je nach Kontext unterschiedlich ausgestaltet ist und die Unterschiede relevant für das Handeln traditioneller Autoritäten sowie für die Erklärung von Konflikten sind. Aufgrund dieser Annahme habe ich mich in meinem Forschungsdesign für eine kollektive Fallstudie entschieden. Die Untersuchung von zwei Fällen hat es mir ermöglicht, Unterschiede und Parallelen im Handeln traditioneller Autoritäten in zwei unterschiedlichen traditionellen Herrschaftssystemen zu erforschen und verallgemeinerbare Hypothesen zur Erklärung von Konflikten um Zugang zu Land aufzustellen. Meine Fälle waren die Konflikte um die Landvergabe an die Unternehmen ScanFarm in der Ashanti Region und BioFuel Africa in der Northern Region Ghanas. Meine zweite Annahme war, dass die Ausgestaltung von $\mathrm{Zu}$ gang zu Land in traditionellen Landverwaltungssystemen und deren Veränderung im Zusammenhang mit land grabbing relevant für die Analyse von Konflikten ist. Diese Annahme ergab sich aus dem Verständnis dieser Konflikte als Konflikte um Zugang zu Land und der Erkenntnis, dass Landnutzer*innen Zugang zu Land in traditionellen Landverwaltungssystemen nicht aus- 
schließlich über Besitzverhältnisse erlangen können, sondern über eine Vielzahl rechtebasierter, struktureller und relationaler Mechanismen. Eine Möglichkeit, Veränderungen in der Ausgestaltung des Zugangs zu Land in den Blick zu nehmen, ist es zu untersuchen, inwiefern sich die Kontrolle über den Zugang zu Land verändert. Daran schließt meine dritte Annahme an, dass die Fähigkeit, Zugang zu Land zu kontrollieren, eine Frage von Macht ist. Die zweite und dritte Annahme waren zentral für die Entwicklung meines Analyserahmens. In diesem habe ich aufbauend auf der Theory of Access (Ribot/Peluso 2003) zunächst ein theoretisch begründetes Verständnis von Zugang zu Land in traditionellen Landrechtssystemen entwickelt. Zugang zu Land zu haben, bedeutet, einen wie auch immer gearteten Nutzen aus dem Land ziehen zu können. Für meinen Analyserahmen habe ich eine Typologie rechtebasierter, relationaler und struktureller Mechanismen erstellt, über die Landnutzer*innen in traditionellen Landverwaltungssystemen Zugang zu Land erlangen können. Um die Veränderung von Zugang zu Land, zu untersuchen, richtete ich den Fokus auf den Wandel von Kontrolle über Zugang zu Land. Die Fähigkeit Kontrolle über den Zugang zu Land ausüben zu können, bedeutet den eigenen Zugang sowie auch den Zugang anderer bestimmen zu können und ist eine Frage von Macht. Mit dem Ziel analysieren zu können, über welche Handlungsstrategien es Akteuren im Kontext von land grabbing gelingt, Zugang zu Land zu kontrollieren, und wie sich diese Fähigkeit erklären lässt, habe ich in meinem Analyserahmen das theoretische Verständnis von Zugang zu Land mit Steven Lukes Konzept der sichtbaren, versteckten und unsichtbaren Macht kombiniert (Lukes 2005). Dieses zeichnet sich dadurch aus, dass es sich nicht auf eine einzige, a priori gesetzte Perspektive auf Macht beschränkt, sondern Macht auf drei unterschiedlichen, sich jedoch nicht gegenseitig ausschließenden Ebenen berücksichtigt: auf der (individuellen) Entscheidungsebene, der Ebene institutioneller Verfahrensweisen sowie der Ebene gesellschaftlicher Kräfteverhältnisse. Lukes berücksichtigt in seiner Konzeptualisierung von Macht gleichermaßen Struktur und Handeln sowie ihren gegenseitigen Einfluss aufeinander. In Bezug auf traditionelle Herrschaftssysteme bietet es sich an, da diese sich durch ihre rechtliche und institutionelle Flexibilität, die Verhandelbarkeit sozialer Positionen sowie durch internalisierte kulturelle und soziale Normvorstellungen auszeichnen. Dies erfordert eine Analyse, die nicht eine einzige, sondern unterschiedliche Formen der Macht berücksichtigt und in Verhältnis zueinander setzt.

Anhand meines Analyserahmens habe ich zunächst herausgearbeitet, über welche unterschiedlichen Mechanismen Landnutzer*innen in den For- 
schungsgegenden vor der Landvergabe Zugang zu Land erlangt haben und welche Unterschiede zwischen verschiedenen Landnutzer*innengruppen bezüglich des Zugangs zu Land bestanden. Aufbauend darauf habe ich untersucht, wie sich durch die Landvergabe die Möglichkeiten der unterschiedlichen Landnutzer*innen weiterhin auf diese Zugangsmechanismen zurückzugreifen, verändert haben, und welche Konsequenzen die Veränderungen für ihren Zugang zu Land hatten. Um Erklärungsansätze zum Zusammenhang zwischen dem Handeln traditioneller Autoritäten und Konflikten um Zugang zu Land entwickeln zu können, habe ich anschließend die Veränderung von Kontrolle über Zugang zu Land in den Blick genommen. Ich habe analysiert, über welche Handlungsstrategien es traditionellen Autoritäten im Kontext der Landvergabe an ScanFarm und BioFuel Africa gelungen ist, Kontrolle über den Zugang zu Land auszuüben. Mithilfe meines Analyserahmens habe ich herausgearbeitet, inwiefern ihr Handeln als Ausübung von Macht $\mathrm{zu}$ verstehen ist. Unter Berücksichtigung der sichtbaren, versteckten und unsichtbaren Macht habe ich typische Handlungsstrategien identifiziert, über die traditionelle Autoritäten in beiden Fällen Zugang zu Land im Zusammenhang mit land grabbing kontrolliert haben. Schließlich habe ich analysiert, wie sich Konflikte um Zugang zu Land im Spannungsfeld der drei Machtformen erklären lassen und inwiefern sich das Handeln traditioneller Autoritäten auf die Entstehung sowie den Verlauf des jeweiligen Konflikts ausgewirkt hat.

Im weiteren Verlauf dieses Kapitels fasse ich die zentralen Ergebnisse meiner Analyse zusammen, diskutiere Unterschiede sowie Parallelen zwischen den beiden Fällen und entwickle mögliche Erklärungsansätze dafür. Anschließend arbeite ich heraus, welche allgemeinen Erkenntnisse sich aus meiner Forschung in Bezug auf traditionelle Herrschaft, land grabbing und Konflikte um Zugang zu Land ableiten lassen. Auf der Grundlage dieser Erkenntnisse entwickle ich Hypothesen zur Erklärung von Konflikten um Zugang zu Land, die in traditionellen Landverwaltungssystemen im Zusammenhang mit land grabbing auftreten. Diese Hypothesen diskutiere ich hinsichtlich ihrer Reichweite und Übertragbarkeit auf andere Länderkontexte. Abschließend zeige ich auf zu welchen Forschungsfeldern ich mit diesem Buch einen Beitrag leiste. 


\subsection{Zusammenfassung und Diskussion der empirischen Erkenntnisse}

Im ersten Analysekapitel (Kapitel 7) habe ich herausgearbeitet, wie Zugang zu Land in beiden Fällen vor der Vergabe des Lands an ScanFarm und BioFuel Africa ausgestaltet war und wie er sich im Kontext von land grabbing verändert hat. Anhand meiner empirischen Daten konnte ich zeigen, dass vor der Landvergabe an die Unternehmen in beiden Gegenden unterschiedliche Akteure den Zugang zu Land kontrollierten: traditionelle Autoritäten sowie indigene Landnutzer*innen, die über permanente Landrechte verfügten. Sie entschieden darüber, zu welchem Zweck und durch wen Land genutzt wurde. Traditionelle Autoritäten übten Kontrolle über den Zugang zu community land und stool land in ihrem Zuständigkeitsbereich aus. Landnutzer*innen mit permanenten Rechten hingegen kontrollierten den Zugang zu ihrem eigenen Land. In beiden Gegenden erhoben im Zuge der Landvergabe an die Unternehmen die in der Hierarchie am höchsten stehenden paramount chiefs den Anspruch, die Eigentümer des Lands (owner of the land) zu sein, obgleich die ihnen untergebenen sub-chiefs zuvor den Zugang zu Land in ihrem Zuständigkeitsgebiet eigenständig kontrollierten. Sie vergaben Land an Mitglieder der Gemeinschaft sowie an migrantische Landnutzer*innen.

Auch bezüglich der Mechanismen, über die Landnutzer*innen vor der Landvergabe an ScanFarm und BioFuel Africa Zugang zu Land erlangten, wiesen die beiden Fälle Parallelen auf. In beiden Gegenden waren rechtebasierte Mechanismen zentral für den Zugang zu Land. Rechtebasierte Mechanismen statten Landnutzer*innen mit Rechten aus, über die sie $\mathrm{Zu}$ gang $\mathrm{zu}$ Land geltend machen können. $\mathrm{Zu}$ diesen Zugangsmechanismen zählten unterschiedlich ausgestaltete Gewohnheitsrechte sowie individuelle Vereinbarungen wie sharecropping oder Pacht. Auf welche rechtebasierten Mechanismen Landnutzer*innen zurückgreifen konnten, um Zugang zu Land zu erlangen, war in beiden Gegenden abhängig von Kategorien sozialer Zugehörigkeit wie Herkunft, Alter und Geschlecht sowie damit zusammenhängenden Ungleichheitsverhältnissen. Unterschiede in den Möglichkeiten Zugang zu Land zu erlangen, bestanden insbesondere zwischen Migrant*innen und indigenen Landnutzer*innen, aber auch innerhalb dieser Gruppen. Insbesondere die Herkunft der Landnutzer*innen hatte maßgeblich Auswirkungen darauf, welche Rechte sie geltend machen konnten, um Zugang zu Land zu erlangen. Diesbezüglich gab es in beiden Gegenden eine Unterscheidung zwischen Landnutzer*innen, deren Vorfahren aus der Gegend kamen, 
und solchen, die aus anderen Gegenden stammten. Erstere bezeichneten sich selbst als Indigene. Landnutzer*innen aus anderen Gegenden galten als Migrant*innen. Der höchste gewohnheitsrechtliche Anspruch, den indigene Landnutzer*innen in den beiden Gegenden geltend machen konnten, war der customary freehold. Ein customary freehold gewährt Landnutzer*innen uneingeschränkte permanente Rechte an Land. Neben der eigenen Nutzung ermöglichen ein customary freehold es Landnutzer*innen, das Land zu verpachten, sharecropping-Vereinbarungen mit anderen Landnutzer*innen $\mathrm{zu}$ treffen und es zu vererben. Diese permanenten Rechte bleiben laut Gewohnheitsrecht auch bestehen, wenn Landnutzer*innen ihr Land lange Zeit brach liegen lassen und nicht nutzen. Landnutzer*innen ohne permanente Rechte wie Migrant*innen oder indigene Landnutzer*innen ohne customary freehold erlangten in beiden Fällen Zugang zu Land über individuelle Vereinbarungen mit Personen, die Kontrolle darüber ausübten. In beiden Forschungsgegenden erhielten diese Landnutzer*innen Zugang zu Land über den jeweiligen sub-chief. In der Gegend von ScanFarm waren zudem sharecropping- oder Pachtvereinbarungen mit Landnutzer*innen, die über einen customary freehold verfügten, gängige rechtebasierte Zugangsmechanismen.

Relationale und strukturelle Zugangsmechanismen ergeben sich aus den kontextspezifischen Bedingungen, unter denen Landnutzer*innen nach $\mathrm{Zu}$ gang zu Land streben. Sie bezeichnen die Möglichkeiten, die sich aus diesen Bedingungen in Bezug auf den Zugang zu Land ableiten. In beiden Gegenden wirkten sich strukturelle und relationale Mechanismen indirekt auf $\mathrm{Zu}$ gang zu Land aus. Strukturelle und relationale Mechanismen beeinflussten, auf welche rechtebasierten Mechanismen Landnutzer*innen zurückgreifen konnten, um Zugang zu Land zu erlangen. Strukturelle und relationale $\mathrm{Zu}$ gangsmechanismen bildeten die Grundlage für die Einforderung von Rechten an Land und ermöglichten oder beschränkten ihre Aushandlung. Zu diesen Zugangsmechanismen zählten in beiden Gegenden Kategorien sozialer Zugehörigkeit wie Alter, Gender, Klasse und Herkunft. Darüber hinaus waren persönliche Beziehungen, die Mitgliedschaft in Netzwerken und die Aushandlung sozialer und rechtlicher Ordnungen relevante strukturelle und relationale Zugangsmechanismen.

Persönliche Beziehungen waren wichtig für die Aushandlung individueller Vereinbarungen, um Zugang zu Land zu erlangen. Dazu gehörten familiäre Beziehungen, Beziehungen zu traditionellen Autoritäten oder zwischen Migrant*innen und den Mitgliedern der indigenen Gemeinschaft. Welche Rechte Landnutzer*innen über die jeweiligen Vereinbarungen geltend 
machen konnten, hing wiederum mit Kategorien sozialer Zugehörigkeit zusammen. Indigene Landnutzer*innen leiteten aus Vereinbarungen mit traditionellen Autoritäten in beiden Gegenden stärkere Zugangsrechte ab als Migrant*innen. Das bedeutet, dass Indigene im Gegensatz zu Migrant*innen in der Regel keine Abgaben für die Landnutzung zahlen mussten und dass sie Anspruch auf eine andere Fläche erheben konnten, wenn die ursprünglich genutzte für andere Zwecke benötigt wurde. Landnutzer*innen, die über permanente Rechte verfügten, gewährten Indigenen und Migrant*innen im Rahmen individueller Vereinbarungen gleiche Rechte. Ein Unterschied zwischen den beiden Gegenden war, dass Frauen in der Northern Region nur über männliche Familienmitglieder Zugang zu individuell genutztem Land erlangten. Dafür verfügten sie unabhängig von männlichen Mitgliedern der Gemeinschaft über starke kollektive Rechte an Gemeinschaftsland, die ihnen Zugang zu Land ermöglichten. In der Ashanti Region hingegen hatten Frauen ebenso wie Männer permanente Rechten an Land. Eine Erklärung für diesen Unterschied ist die Varianz in der Erbfolge in beiden Regionen. Das traditionelle Herrschaftssystem der Ashanti ist matrilineal, was bedeutet, dass die Erbschaft über die mütterliche Blutlinie erfolgt. Das kann bedeuten, dass Männer ihr Land an die Söhne ihrer Schwester statt an ihre eigenen Kinder vererben. Üblich ist aber auch die Weitergabe des Lands von Mutter zu Tochter, was Frauen individuelle Landrechte zusichert (Amanor 2008; Berge et al. 2014; Peters 2010). Das traditionelle Herrschaftssystem der Dagombas in der Northern Region hingegen ist patrilineal. Ein weiterer Unterschied zwischen den beiden Fällen war die Bedeutung der Aushandlung sozialer und rechtlicher Ordnungen als Teil der strukturellen und relationalen Mechanismen. Sie erwies sich vor allem im Fall von ScanFarm als relevant für Zugang zu Land. Landnutzer*innen erhoben dort über zwei unterschiedliche Argumentationsweisen Anspruch auf permanente Landrechte. Wie in der Northern Region forderten sie permanente Rechte über das Argument der ersten Niederlassung (first settlement) ein. Weitaus häufiger leiteten Landnutzer*innen ihre Rechte jedoch daraus ab, dass sie oder ihre Vorfahren die ersten waren, die das Land bestellt haben (first cultivation). Auf diese Möglichkeit, permanente Landrechte einzufordern, griffen Landnutzer*innen in der Northern Region nicht zurück. Eine Erklärung für diesen Unterschied bietet die Art der vorherigen Landnutzung. Ein Großteil des durch ScanFarm beanspruchten Lands ist Waldgebiet. In diesem Gebiet begannen in den 1970er Jahren Kleinbäuerinnen und -bauern - unter ihnen auch zahlreiche Migrant"innen - mit der Erlaubnis des paramount chiefs Kakaoplantagen anzulegen. Diese Tätigkeit er- 
forderte hohe finanzielle und zeitliche Investitionen. Aufgrund dieser Investitionen erhoben Landnutzer*innen fortan Ansprüche auf dieses Land. Auch wenn die Kakaoplantagen heute nicht mehr vorhanden sind und die Landnutzer*innen das Land anders nutzen, bestehen die Ansprüche auf das Land fort. In der Gegend von BioFuel Africa hat keine vergleichbare Entwicklung stattgefunden. Das Land dort ist größtenteils bislang ungerodetes, dünn besiedeltes Buschland, jedoch keinesfalls ungenutzt. Unter Berücksichtigung der vorherrschenden traditionellen Rechte und Normen nutzen die dort lebenden Landnutzer*innen das Land für vielfältige Zwecke. Das gleiche Stück Land wird mitunter gleichzeitig oder zeitlich versetzt auf unterschiedliche Weise genutzt: als Weidefläche, zum Sammeln von Feuerholz oder wildwachsender Pflanzen, für die Jagd etc. Das erklärt, warum die Rodung und damit verbundene vermeintliche Erstbestellung von Land hier Landnutzer*innen nicht die Möglichkeit bietet, permanente Rechte einzufordern. Dieser Anspruch würde zum Ausschluss vieler anderer Nutzer*innen führen.

Im Zusammenhang mit land grabbing veränderten sich in beiden Fällen die Möglichkeiten der Landnutzer*innen, Zugang zu Land aufrechtzuerhalten oder $\mathrm{zu}$ erlangen. Insbesondere gingen die Landvergaben an ScanFarm und BioFuel Africa mit einer Zentralisierung der Kontrolle über den Zugang zu Land einher. In beiden Fällen verliefen die Verhandlungen über die Vergabe des Lands bilateral zwischen dem paramount chief und den Vorsitzenden der Unternehmen. Die sub-chiefs und Landnutzer*innen mit permanenten traditionellen Rechten, die zuvor Zugang zu Land kontrollierten, waren von den Verhandlungen ausgeschlossen. Somit konnten sie nicht über die Nutzung des Lands durch die Unternehmen mitentscheiden. Sie verloren die Kontrolle über den Zugang zu ihrem Land an den jeweiligen paramount chief als alleinigen Verhandlungspartner der Unternehmen. Diese Entwicklung bezeichne ich als Zentralisierung der Kontrolle.

Der Verlust von Kontrolle über den Zugang zu Land führte jedoch nicht für alle Landnutzer*innen gleichermaßen zum Verlust ihres Zugangs zu Land. Einige Landnutzer*innen verloren ihren Zugang zu Land vollständig, während die Landvergabe für andere eine maßgebliche Einschränkung ihres Zugangs zur Folge hatte. Einschränkungen äußerten sich darin, dass der Nutzen, den Landnutzer*innen nach der Landvergabe aus dem Land ziehen konnten, geringer war als vor der Landvergabe. Im Allgemeinen verringerten sich im Zusammenhang mit der Landvergabe an die Unternehmen die Möglichkeiten, über die Landnutzer*innen ihren Zugang zu Land (aufrecht)erhalten konnten. 
Die Möglichkeiten der Landnutzer*innen ihren Zugang zu Land nach der Landvergabe aufrechtzuerhalten, standen in einem Zusammenhang damit, inwiefern ihre traditionellen Landrechte in den Verhandlungen mit den Unternehmen Anerkennung fanden. In den Verhandlungen über die Landvergabe an ScanFarm berücksichtigten die verhandelnden Parteien - der paramount chief und die Vorsitzenden des Unternehmens - die traditionellen Landrechte der Landnutzer*innen nicht, so dass die Landvergabe an ScanFarm für die betroffenen Landnutzer*innen zum Verlust ihres Zugangs zu Land führte. Diesbezüglich unterschied sich die Landvergabe an BioFuel Africa, in der die traditionellen Landrechte indigener Landnutzer*innen zum Teil Berücksichtigung durch das Unternehmen fanden. BioFuel Africa erklärte sich bereit, bestellte Felder um die ausgewiesenen Dörfer - wenn möglich - von den Rodungsarbeiten $\mathrm{zu}$ verschonen oder anderenfalls alternative Felder für die Landnutzer*innen bereitzustellen. Kollektive Landrechte und Rechte von migrantischen Landnutzer*innen berücksichtigte das Unternehmen jedoch nicht. Diese Unterschiede lassen sich insbesondere über das Handeln der Unternehmen erklären. Im Rahmen der Landvergabe an BioFuel Africa forderte das Unternehmen einen Austausch mit den auf dem Land lebenden Landnutzer*innen ein, um sich über bestehende Rechte und die Nutzung des Lands zu informieren. In den Verhandlungen über die Landvergabe an ScanFarm forderten die Vorsitzenden des Unternehmens diesen Austausch nicht ein.

Letztendlich führte die Landvergabe in beiden Fällen zu einer Einschränkung und Schwächung des Zugangs für alle Landnutzer*innen, wodurch es zu Konflikten kam. Insbesondere rechtebasierte Zugangsmechanismen verloren an Bedeutung. Traditionelle Landrechte wurden im Zusammenhang mit der Landvergabe irrelevant und ermöglichten es Landnutzer*innen nicht, ihren Zugang zu Land aufrechtzuerhalten. Strukturelle und relationale Mechanismen wurden zum Teil wichtiger, um Zugang zu Land erlangen zu können, teilweise verloren sie aber auch an Bedeutung. Eine Erklärung dafür ist die Verschiebung der Zugangskontrolle. Gute Beziehungen zum paramount chief erwiesen sich in beiden Fällen als förderlich für den Zugang zu Land. Persönliche Beziehungen zu den sub-chiefs oder Landnutzer*innen mit permanenten Rechten, die zuvor Zugang zu Land über Pacht- oder sharecropping-Vereinbarungen ermöglicht hatten, verloren im Zusammenhang mit land grabbing ihre Bedeutung.

Ebenfalls an Relevanz gewannen Kategorien sozialer Zugehörigkeit. Insbesondere die Unterscheidung zwischen Indigenen und Migrant*innen wurde wichtiger. Indigene Landnutzer*innen hatten in beiden Fällen die Mög- 
lichkeit, eine Entschädigung für den Verlust ihres Lands einzufordern. ScanFarm leistete Entschädigungszahlungen an indigene Landnutzer*innen, BioFuel Africa stellte indigenen Landnutzer*innen für den Verlust ihres Lands alternative Flächen zur Verfügung. Selbst in diesen Fällen verschlechterte sich jedoch der Zugang zu Land. Die Entschädigungszahlungen waren geringer als das Einkommen, das Landnutzer*innen zuvor mit dem Land erwirtschaftet hatten, und die durch BioFuel Africa zur Verfügung gestellten alternativen Felder waren schwer zu erreichen. Frauen, die zuvor Zugang zu Land über kollektive Landrechte hatten, erhielten keine Entschädigung. Gleiches gilt für Migrant*innen. Diejenigen, die bereits vor der Landvergabe über schwache Rechte verfügten, waren am stärksten vom vollständigen Verlust des Zugangs betroffen. Die Veränderung der Kontrolle über Zugang zu Land, die im Zusammenhang mit land grabbing zu beobachten ist, hat also zu einer Verstärkung von Hierarchien und sozialer Ungleichheit geführt.

In beiden Fällen traten im Zusammenhang mit der Vergabe des Lands an die Unternehmen ScanFarm und BioFuel Africa Konflikte auf. Die Konflikte entstanden aufgrund der Veränderung des Zugangs zu Land für die Landnutzer*innen, zu der es im Zusammenhang mit der Landvergabe kam. Die Akteure waren in beiden Konflikten Mitarbeitende der Unternehmen, Landnutzer*innen und traditionelle Autoritäten. Landnutzer*innen und traditionellen Autoritäten sind dabei als heterogene Gruppen zu verstehen, in denen die einzelnen Akteure sozial differenziert sind. Unterschiede zwischen den Akteuren bestanden insbesondere hinsichtlich ihrer Möglichkeiten Zugang zu Land zu erlangen oder ihn zu kontrollieren. In der Analyse der Konflikte hat sich insbesondere die Hierarchie zwischen sub-chiefs und paramount chiefs als relevant erwiesen sowie die Differenzierung der Landnutzer*innen hinsichtlich Kategorien sozialer Zugehörigkeit wie Geschlecht, Alter, Herkunft, Bildungsstand und Klasse.

Im Mittelpunkt des Konflikts um Zugang zu Land im Zusammenhang mit der Landvergabe an ScanFarm stand die intransparente Verhandlung der Landvergabe zwischen dem paramount chief und den Vorsitzenden des Unternehmens. Die Intransparenz entstand durch die mangelnde Information, Konsultierung und Partizipation von Landnutzer*innen und sub-chiefs. Für die Landnutzer"innen und sub-chiefs bedeutete dieser Ausschluss aus den Verhandlungen, dass sie nicht für ihre Interessen eintreten konnten, keinen Einfluss auf Entscheidungen bezüglich ihres Zugangs zu Land hatten und erst von der Landvergabe erfuhren, als das Unternehmen begann das Land zu bearbeiten. Die Folge war der Verlust des Zugangs zu Land und damit einher- 
gehend die Verschlechterung ihrer Lebensgrundlage oder ihres Einkommens. Ziel der Landnutzer“innen war es daher, den Zugang zu ihrem Land, zurückzugewinnen. Mit dieser Forderung wandten sie sich an den paramount chief. Dieser behauptete, es sei nicht seine Intention gewesen, mehr als nur sein eigenes stool land an das Unternehmen zu vergeben und dem Ganzen liege ein Missverständnis zugrunde. Die Vorsitzenden des Unternehmens beriefen sich jedoch auf ihr Abkommen mit dem paramount chief. Laut diesem Abkommen habe der paramount chief ihnen die Erlaubnis zur Nutzung des Lands erteilt. Obwohl der paramount chief behauptete, es handele sich um ein Missverständnis, setzte er sich nicht dafür ein, den Landnutzer*innen wieder $\mathrm{Zu}$ gang zu ihrem Land zu ermöglichen. Statt des Zugangs zu Land stellte der paramount chief den Landnutzer"innen und sub-chiefs zahlreiche Vorteile, die das Vorhaben mit sich bringen sollte, in Aussicht. $\mathrm{Zu}$ diesen zählten unter anderem die Verbesserung des Zugangs zu Wasser, der Gesundheitsversorgung und der Straßen sowie der Bau einer Schule. Zudem empfahl er den Landnutzer*innen und sub-chiefs von ScanFarm Entschädigungszahlungen für den Verlust ihres Landes oder ihrer Ernte einzufordern. Ein Großteil der Landnutzer*innen nahm die Aushandlung von Entschädigungszahlungen als einzige Handlungsoption wahr. Im weiteren Verlauf verlagerte sich der Konflikt daher insbesondere auf die Verhandlung der Höhe der Entschädigungszahlungen. Unerfüllte Erwartungen seitens der Landnutzer"innen und sub-chiefs hinsichtlich der Entschädigungshöhe sowie der vermeintlichen Vorteile des Projekts verstärkten den Konflikt. Es kam zu Protesten und Beschwerden der Landnutzer"innen und sub-chiefs beim paramount chief gegen das Vorhaben von ScanFarm. Einige Landnutzer*innen erhoben Anklage beim Kumasi High Court gegen das Unternehmen und versuchten den Zugang zu ihrem Land über das staatliche Rechtssystem wiederzuerlangen.

Im Kern des Konflikts verbunden mit der Landvergabe an BioFuel Africa ging es ebenfalls um die Veränderung des Zugangs zu Land. Auch hier entschied der paramount chief allein über die Vergabe des Lands. Die Verhandlungen darüber verliefen bilateral zwischen ihm und den Vorsitzenden des Unternehmens. Im Unterschied zur Landvergabe an ScanFarm informierten die beiden Verhandlungspartner jedoch die indigenen Landnutzer"innen und sub-chiefs über das Vorhaben. Das führte, anders als in den Verhandlungen um die Landvergabe an ScanFarm, zunächst zu einer größeren Transparenz. Dennoch traten im Verlauf der Umsetzung des Vorhabens durch BioFuel Africa auch hier Konflikte auf. Zentrale Themen in diesen Konflikten waren die Verschlechterung der Lebensgrundlange der Landnutzer"innen durch den Ver- 
lust oder die Einschränkung des Zugangs zu Land; Veränderungen der Umgebung durch die Arbeiten des Unternehmens und damit verbundene negative Folgen für das Leben in der Gegend; die besondere Betroffenheit von Frauen; Ungleichheiten in der Verteilung von Arbeitsplätzen durch das Unternehmen; der mangelnde Informationsfluss zwischen dem Unternehmen und den Landnutzer*innen sowie zwischen unterschiedlichen traditionellen Autoritäten; und unerfüllte Versprechen seitens des Unternehmens. Auch in diesem Fall wandten sich die sub-chiefs als Vertreter ihrer Gemeinschaft mit ihren Beschwerden an den paramount chief. Auch dieser legte ihnen die vermeintlichen Vorteile des Vorhabens von BioFuel Africa dar, um sie trotz ihrer Kritik von dem Projekt zu überzeugen. Anders als im Konflikt um die Landvergabe an ScanFarm beschränkten sich die Austragungsformen des Konflikts auf Beschwerden beim paramount chief sowie die Erregung medialer Aufmerksamkeit über Radiosendungen durch eine kleine Gruppe von Landwirt*innen, die selbst nicht in der Gegend lebten, sondern täglich aus der Regionalhauptstadt kamen, um dort Landwirtschaft zu betreiben.

In Kapitel 8 habe ich die Entstehung und den Verlauf dieser Konflikte mit einem Fokus auf das Handeln traditioneller Autoritäten untersucht. Ich habe analysiert, wie es den paramount chiefs gelang, sub-chiefs und Landnutzer*innen die Kontrolle über den Zugang zu Land zu entziehen. Dafür habe ich typische Handlungsstrategien identifiziert und mit Hilfe meines Analyserahmens herausgearbeitet, inwiefern diese Handlungsstrategien als Ausübung sichtbarer, versteckter oder unsichtbarer Macht verstanden werden können. Anschließend habe ich untersucht, wie sich die Ausübung der unterschiedlichen Machtformen auf die Entstehung und den weiteren Verlauf der Konflikte ausgewirkt hat. Über meine Analyse konnte ich zeigen, dass die paramount chiefs in beiden Fällen kaum sichtbare Macht, sondern in erster Linie versteckte Macht ausübten, um den Zugang zu Land kontrollieren zu können. Auch in ihren Handlungsstrategien konnte ich Parallelen herausarbeiten. Typische Handlungsstrategien waren die Einflussnahme auf institutionelle Verfahrensweisen, die Neuinterpretation bestehender traditioneller Regeln und Normen sowie die Kontrolle von Informationen gegenüber subchiefs, Landnutzer*innen und Mitarbeiter*innen von Behörden. Die unterschiedlichen Formen der versteckten Macht ermöglichten den paramount chiefs diese Akteure aus den Verhandlungen der Landvergabe auszuschließen und als alleinige Verhandlungspartner aufzutreten. Neben der versteckten Macht erwies sich auch die unsichtbare Macht als zentral in Bezug auf das Handeln traditioneller Autoritäten. Die unsichtbare Macht der paramount chiefs stärkte 
in beiden Fällen ihre Fähigkeiten, versteckte Macht auszuüben. Unsichtbare Macht bedeutet, dass Akteure das Handeln entgegen ihrer ethischen Interessen hinnehmen, sich also nicht dagegen wehren oder sogar unterstützend daran mitwirken. Die unsichtbare Macht der paramount chiefs beruht auf ihrer Anerkennung durch die Gemeinschaft. Diese ist wiederum die Voraussetzung für ein Leben in der Gemeinschaft. Im Fall von ScanFarm kam die unsichtbare Macht der paramount chiefs unter anderem darin zum Ausdruck, dass die Landnutzer"innen aufhörten Widerstand zu leisten und sich darauf einließen, Entschädigungszahlungen mit dem Unternehmen auszuhandeln, statt ihr Land zurückzufordern, obwohl sich dadurch ihre Lebensbedingungen verschlechterten. Auch im Fall von BioFuel Africa äußerte sich die unsichtbare Macht des paramount chiefs im fehlenden Widerstand der Landnutzer*innen und sub-chiefs gegen die Landvergabe und das Vorhaben des Unternehmens. Aufgrund gesellschaftlicher Normen akzeptierten sie, sich nicht gegen die Entscheidung des paramount chiefs stellen zu können. Das Handeln des paramount chiefs verletzte ihre ethischen Interessen, doch sie erkannten ihr fehlendes Mitspracherecht als konform mit den traditionellen Regeln ihrer Gemeinschaft an. Daher leisteten auch sie keinen Widerstand, obwohl sie die Missstände, die die Landnutzung durch BioFuel Africa zur Folge hatte, klar benennen konnten.

Bezüglich des Zusammenhangs zwischen dem Handeln der paramount chiefs und der Entstehung sowie dem Verlauf von Konflikten um Zugang konnte ich über meine Analyse folgende Erkenntnisse gewinnen. Die versteckte Macht, die unter anderem in der mangelnden Information, Konsultierung und Partizipation der sub-chiefs und Landnutzer*innen zum Ausdruck kam, verhinderte in beiden Fällen zunächst die Entstehung eines Konflikts. Bevor sich der Zugang zu ihrem Land durch die Arbeiten der Unternehmen änderte, erkannten die Landnutzer"innen und sub-chiefs aufgrund mangelnder Informationen noch nicht, dass die paramount chiefs mit der Landvergabe entgegen ihrer Interessen handelten. Später, als sich Landnutzer*innen und sub-chiefs der Veränderung ihres Zugangs zu Land bewusst wurden und sich mit ihren Beschwerden darüber an die paramount chiefs wandten, kam deren mangelnde Rechenschaftspflicht gegenüber ihren Gemeinschaften zum Ausdruck. Das Handeln entgegen der Interessen ihrer Gemeinschaften hatte für die paramount chiefs keine (negativen) Konsequenzen. Landnutzer*innen und sub-chiefs standen zudem keine Handlungsmöglichkeiten zur Verfügung, um gegen das Handeln des paramount chiefs vorzugehen oder ihre Rechte auf $\mathrm{Zu}$ gang zu Land durchzusetzen. Aufgrund der versteckten Macht waren offe- 
ner Widerstand und die Herausforderung der Entscheidungen der paramount chiefs keine Option. Und auch darüber hinaus existierten keine (institutionalisierten) Wege, ihre Rechte an Land geltend $\mathrm{zu}$ machen oder traditionelle Autoritäten außerhalb des traditionellen Herrschaftssystems zur Verantwortung dafür zu ziehen, entgegen den Interessen der Gemeinschaft gehandelt zu haben.

In Bezug auf die Ausübung sichtbarer Macht - der Durchsetzung der eigenen Interessen auch gegen Widerstreben - wird in der Analyse der beiden Konflikte deutlich, dass diese nicht zu den typischen Handlungsstrategien traditioneller Autoritäten zählt. Insbesondere mit Blick auf die Entstehung und den Verlauf von Konflikten ist das interessant. Indem die paramount chiefs nicht offen gegen die Interessen der sub-chiefs und Landnutzer*innen handelten, vermieden sie auch die direkte Konfrontation mit ihnen. Wann immer sich der Konflikte manifestierte und die Landnutzer*innen und subchiefs Missstände, gegensätzliche Interessen oder verletzte Bedürfnisse kommunizierten, versuchten die paramount chiefs zunächst - auf unterschiedliche Weise - sie zu beschwichtigen und Lösungsoptionen vorzuschlagen. Damit gelang es ihnen, eine Eskalation zu vermeiden. Hinsichtlich der verletzten Interessen sind die Lösungsoptionen jedoch nicht auf die Bedürfnisse der Landnutzer*innen ausgerichtet, sondern sie zielen vielmehr auf eine technokratische Regelung des Konflikts ab. Statt die Landvergabe als solche in Frage zu stellen, fokussieren die Lösung von Streitpunkten, die erst durch die Landvergabe entstanden sind. Dazu zählt unter anderem die Verhandlung Entschädigungszahlungen. Damit geht in beiden Fällen eine Verschiebung in der Austragung des Konflikts einher. Der Fokus der sub-chiefs und Landnutzer*innen wechselt vom Handeln der paramount chiefs auf das Handeln der Unternehmen. Obwohl die Konflikte weiterhin bestehen bleiben, ist die Konsequenz daraus eine geringere Konfrontation zwischen paramount chiefs und den landnutzenden Gemeinschaften.

Die empirischen Erkenntnisse, die der Vergleich der beiden Fälle bezüglich der Erklärung von Konflikten liefert, zeigen klare Parallelen, aber auch Unterschiede auf. Im folgenden Abschnitt diskutiere ich diese.

Im Zentrum beider Konflikte stehen die antagonistischen Interessen der Konfliktakteure in Bezug auf den Zugang zu Land. Insbesondere über unterschiedliche Formen versteckter Macht gelang es den paramount chiefs in beiden Fällen, sich die Kontrolle über den Zugang zu Land anzueignen und diese an die Unternehmen zu übertragen. Die Konflikte, die damit einhergingen, verliefen zwischen denjenigen, die sich die Kontrolle über den $\mathrm{Zu}$ - 
gang zu Land neu angeeignet haben (paramount chief und Unternehmen) und denjenigen, die ihren Zugang zu Land oder die Kontrolle über den Zugang $\mathrm{zu}$ Land verloren haben (Landnutzer*innen und sub-chiefs). In beiden Fällen entstanden die Konflikte zu dem Zeitpunkt, zu dem Landnutzer*innen und sub-chiefs Kenntnis über die Einschränkungen oder den Verlust ihres Zugangs zu Land erlangten. Eine weitere Parallele besteht hinsichtlich der Manifestierung der Konflikte durch die Kommunikation der antagonistischen Interessen. In beiden Fällen wandten sich die Landnutzer*innen zunächst mit ihren Beschwerden über die Einschränkung oder den Verlust ihres Zugangs zu Land an den sub-chief, der die Anliegen an den jeweiligen paramount chief weiterleitete. Die paramount chiefs positionierten sich trotz der Beschwerden zugunsten der Landvergabe. Sie versuchten die Landnutzer"innen und sub-chiefs von dem Vorhaben zu überzeugen, indem sie vermeintliche Vorteile in den Vordergrund stellten. Die Parallelen zu Beginn der beiden Konflikte lassen sich über die gleichen rechtlichen Rahmenbedingungen, unter denen die Vergabe des Lands stattfand, erklären. Von Bedeutung diesbezüglich ist insbesondere das Verhältnis von traditioneller zu staatlicher Herrschaft (Berry 2017; Boone 2015), das in beiden Fällen durch die nationale Verfassung (Republic of Ghana 1992) bestimmt ist. Angelegenheiten, die traditionell verwaltetes Land betreffen, unterliegen laut Verfassung der alleinigen Hoheit traditioneller Autoritäten, was ihre zentrale Rolle zu Beginn beider Konflikte erklärt.

Trotz der anfänglichen Parallelen sind die Konflikte in ihrem weiteren Verlauf durch unterschiedliche Forderungen sowie unterschiedliche Handlungsstrategien der Konfliktakteure gekennzeichnet. Im Fall von ScanFarm richteten die Landnutzer*innen und sub-chiefs nach dem Ersuch des paramount chiefs ihre Forderungen insbesondere an das Unternehmen und teilweise auch an staatliche Institutionen. Sie verfassten Briefe an das Unternehmen und suchten das Gespräch mit dem Geschäftsführer des Unternehmens, um ihren Zugang zu Land zurückzufordern sowie später Entschädigungszahlungen auszuhandeln. Einige weigerten sich ihr Land zu verlassen und es dem Unternehmen zu überlassen und leisteten - teilweise mit der Androhung von Waffengewalt - Widerstand gegen die Nutzung ihres Lands durch das Unternehmen. Darüber hinaus erstattete ein Teil der Landnutzer*innen Anzeige gegen die Nutzung des Lands durch ScanFarm bei der lokalen Polizei und zog vor Gericht. Zudem fanden zu Beginn des Konflikts - unterstützt durch ActionAid Ghana - allgemeine Proteste gegen land grabbing statt. An diesen Protesten - die vor dem Agogo Traditional Council endeten - nahmen nicht nur 
von der Landvergabe betroffene Landnutzer*innen teil, sondern auch andere Einwohner*innen von Agogo.

Im Fall von BioFuel Africa war der Verlauf des Konflikts nach dem Ersuch des paramount chiefs durch die Abwesenheit konfrontativer Handlungsstrategien gekennzeichnet. Landnutzer*innen sowie sub-chiefs tauschten sich untereinander über ihre Missstände aus, jedoch stellten sie keine Forderungen an den paramount chief oder das Unternehmen. Eine Ausnahme bildete eine kleine Gruppe Landwirt*innen, die nicht in der Gegend lebten, sondern nur Land dort nutzten. Sie kämpften gegen den Verlust ihres Lands, indem sie ebenfalls mit Unterstützung durch ActionAid Ghana - über Radiosendungen zu der Landvergabe versuchten mediale Aufmerksamkeit für land grabbing in Ghana und insbesondere die Landvergabe an BioFuel Africa zu erregen.

Das Handeln der paramount chiefs bietet - über die Ausübung unterschiedlicher Formen der Macht und der Aneignung der Kontrolle über Zugang zu Land hinaus - eine mögliche Erklärung für die Unterschiede im Verlauf der beiden Konflikte. Im Fall von ScanFarm forderte der paramount chief zu Beginn der Verhandlungen die Zahlung von drink money durch das Unternehmen ein. Die Höhe der Summe behielt er für sich und teilte die Einnahmen auch nicht mit den landnutzenden Gemeinschaften. Den Ausschluss der Landnutzer*innen von der Teilhabe an den Einnahmen begründete er im Nachhinein damit, dass er nur sein eigenes stool land vergeben habe. Vor diesem Hintergrund wies er die Verantwortung für die Aneignung des Lands der Landnutzer*innen durch ScanFarm im Gespräch mit den sub-chiefs und Landnutzer*innen von sich. Dem Unternehmen erteilte er jedoch später die schriftliche Erlaubnis, auch bei Widerstand seitens der Landnutzer*innen mit den Arbeiten auf dem Land fortzufahren. Die Landnutzer*innen verwies er mit ihren Beschwerden an das Unternehmen. Dieses Vorgehen erklärt, warum die Landnutzer*innen ihre Forderungen im weiteren Verlauf des Konflikts an ScanFarm und nicht mehr an den paramount chief richteten. Im Konflikt mit dem Unternehmen war eine der wenigen Handlungsoptionen, die sich den Landnutzer*innen bot, die Verhandlung von Entschädigungszahlungen. Die Landvergabe an BioFuel Africa zeichnete sich - trotz mangelnder Partizipation der Landnutzer*innen und sub-chiefs in den Verhandlungen - durch eine höhere Transparenz aus. Unter anderem war die Höhe der Summe, die das Unternehmen für die Nutzung des Lands leistete, öffentlich bekannt und der paramount chief ließ alle indigenen Gemeinschaften in der Projektgegend daran teilhaben. Hinzu kam, dass BioFuel Africa im Vorfeld Absprachen mit den Landnutzer*innen bezüglich ihrer Felder traf. Das Unternehmen erklärte 
sich bereit, die Felder der Landnutzer*innen bei der Errichtung der JatrophaPlantage wenn möglich unberührt zu lassen oder ihnen alternative Flächen zur Verfügung zu stellen. Der Ablauf der Landvergabe und das Vorgehen des Unternehmens liefern eine Erklärung für spätere Unterschiede. Da die Felder der Landnutzer*innen bei der Errichtung der Jatropha-Plantage unberührt bleiben sollten, war die Einforderung individueller Entschädigungszahlungen in diesem Fall keine Handlungsoption.

Der unterschiedliche Verlauf der Konflikte, der unter anderem in unterschiedlichen Forderungen und Handlungsstrategien der Landnutzer*innen zum Ausdruck kommt, lässt sich somit nicht nur über das Handeln der paramount chiefs erklären. Die empirischen Erkenntnisse deuten darauf hin, dass neben dem Handeln der paramount chiefs weitere Faktoren relevant für die Erklärung der Konflikte sind. Teilweise beeinflusst das Handeln der paramount chiefs diese Faktoren; teilweise sind sie unabhängig davon zu betrachten. $\mathrm{Zu}$ den weiteren Faktoren zählen, wie ich nachfolgend erläutern werde, das Handeln der Unternehmen und der Ablauf der Landvergabe, die soziale Zugehörigkeit der Landnutzer*innen und ihre Möglichkeiten, Zugang zu Land zu erlangen, sowie die Entfernung der durch die Landvergabe betroffenen Dörfer von größeren Städten und dem Sitz der Unternehmen ScanFarm und BioFuel Africa.

Über den Vergleich der beiden Fälle werden Unterschiede hinsichtlich der Landvergabe deutlich. Meine erhobenen Daten zeigen, dass sich trotz gleicher rechtlicher Rahmenbedingungen die Landvergabe an ScanFarm in ihrem Ablauf von derjenigen an BioFuel Africa unterschied. Die Landvergabe an BioFuel Africa zeichnete sich im Vergleich zur Landvergabe an ScanFarm durch eine frühere sowie stärkere Einbeziehung von Behörden und durch die vorherige Informierung eines Teils der - vornehmlich indigenen - sub-chiefs und Landnutzer*innen aus. Dadurch genoss das Projekt später eine höhere Legitimität, die sich darin äußerte, dass sub-chiefs und Landnutzer"innen die Landvergabe nicht per se infrage stellten. Sie bemängelten vornehmlich enttäuschte Erwartungen oder unerwartete Folgen des Vorhabens. Die Landvergabe an ScanFarm verlief anders. Zentral waren der absolute Ausschluss der sub-chiefs und Landnutzer*innen sowie zunächst auch die Abwesenheit von Behörden. Im dem Konflikt, der in diesem Kontext entstand, stellten Landnutzer*innen die Landvergabe im Allgemeinen und insbesondere ihre Rechtmäßigkeit infrage. Sie reichten Klage gegen das Unternehmen ein und weigerten sich, ihr Land dem Unternehmen zu überlassen. Der Ablauf der Landvergabe und insbesondere (fehlende) Absprachen zwischen Landnutzer*innen und Unternehmen 
wirkten sich auf die Forderungen der Landnutzer*innen aus und bieten eine Erklärung dafür, warum die Verhandlung von Entschädigungszahlungen im einen Fall so prominent waren und im anderen Fall keine Rolle spielten. Die Entscheidungen der Vorsitzenden von ScanFarm und BioFuel Africa bezüglich des Ablaufs der Landvergabe hatten also ebenfalls Einfluss auf den Verlauf der Konflikte. Das Handeln der Unternehmen muss in der Erklärung von Konflikten um Zugang zu Land, die im Zusammenhang mit land grabbing auftreten, mitberücksichtigt werden.

Ein weiterer Faktor, der sich im Vergleich der beiden Fälle als relevant für die Erklärung von Konflikten um Zugang herausgestellt hat, war die soziale Zugehörigkeit der Landnutzer*innen. In beiden Konflikten bestimmte diese, über welche Mechanismen Landnutzer*innen zuvor Zugang zu Land erlangen konnten, bewirkte, dass Akteure auf unterschiedliche Weise durch die Landvergabe betroffen waren, und beeinflusste die Handlungsmöglichkeiten, die ihnen in den Konflikten um Zugang zu Land zur Verfügung standen. In beiden Fällen war die Herkunft der Landnutzer*innen eine zentrale Kategorie sozialer Zugehörigkeit. In den Ergebnissen der Analyse kommt dies insbesondere in Unterschieden zwischen indigenen und migrantischen Landnutzer*innen zum Ausdruck. Im Fall von ScanFarm war zudem die Differenzierung innerhalb der Gruppe der indigenen Landnutzer*innen von Bedeutung, die in unterschiedlichen Rechten an Land Ausdruck fand. Auch Bildung spielte diesbezüglich eine Rolle. Im Fall von BioFuel Africa war hinsichtlich der Differenzierung innerhalb der Gruppe der indigenen Landnutzer*innen die Kategorie Geschlecht bedeutend in der Analyse der Konflikte. Das hing damit zusammen, dass sich das Unternehmen vornehmlich kollektiv von Frauen genutztes Land aneignete.

Schließlich gibt der Vergleich der beiden Fälle Anhaltspunkte dafür, dass sich neben dem Handeln der paramount chiefs, dem Handeln der Unternehmen und der sozialen Identität der Landnutzer*innen auch die Distanz der durch die Landvergabe betroffenen Dörfern zu nächst größeren Städten und zum Sitz des Unternehmens auf den Verlauf von Konflikten um Zugang zu Land auswirken. Die Projektgegenden von ScanFarm und BioFuel Africa weisen diesbezüglich deutliche Unterschiede auf. Die Projektgegend von ScanFarm liegt in unmittelbarer Nähe der Bezirkshauptstadt Agogo, wo das Unternehmen seinen Geschäftssitz hat. Viele der Landnutzer*innen aus den Dörfern Dukusen, Nsonyameye und Baama wohnen außerhalb der Ernte- und Anbauzeit ebenfalls in Agogo. Die Projektgegend von BioFuel Africa hingegen liegt ca. $50 \mathrm{~km}$ weit entfernt von der Regionalhauptstadt Tamale und dem Sitz des Un- 
ternehmens. Auch zwischen den einzelnen betroffenen Dörfern liegen zum Teil große Distanzen. Neben dem Handeln der paramount chiefs bieten diese Faktoren einen weiteren Erklärungsansatz für den Verlauf von Konflikten. Sie sind relevant um zu verstehen, warum sich die Landnutzer*innen und sub-chiefs im Fall von ScanFarm mit ihren Forderungen an das Unternehmen wenden konnten, im Fall von BioFuel Africa jedoch nicht. Das Aufsuchen der Geschäftsstelle von BioFuel Africa wäre mit hohem zeitlichem Aufwand, hohen Transportkosten und Ortskenntnissen verbunden gewesen. Die Landnutzer*innen von Dukusen, Nsonyameye oder Baama hingegen konnten die Geschäftsstelle von ScanFarm ohne weitere Mühen und Kosten aufsuchen. Auch hinsichtlich der Bildung von Allianzen sind diese Faktoren von Bedeutung. Im Fall von ScanFarm standen die von der Landvergabe betroffenen Landnutzer*innen aus den unterschiedlichen Dörfern in regelmäßigem Austausch. Einige von ihnen kannten sich vor der Landvergabe bereits persönlich aus ihrem gemeinsamen Wohnort Agogo und trugen zu einer guten Vernetzung und Solidarisierung vieler betroffener Landnutzer*innen bei. Im Fall von BioFuel Africa fand dieser Austausch zwischen den Landnutzer*innen aus den unterschiedlichen Dörfern nicht statt. Die große Distanz zwischen den Dörfern ist eine mögliche Erklärung dafür. Der fehlende Austausch erschwert wiederum auch die Solidarisierung der Landnutzer*innen und die gemeinsame Entwicklung von Handlungsstrategien. Die Unterschiede bezüglich der geografischen Lage ihrer Implikationen für das Handeln der Akteure bieten einen möglichen Erklärungsansatz für die unterschiedlich erfolgreiche Mobilisierung der Landnutzer*innen gegen land grabbing durch ActionAid Ghana. In Agogo mobilisierte ActionAid Ghana erfolgreich gegen land grabbing. Gemeinsam mit dem zivilgesellschaftlichen Netzwerk Agogoman mma kuo, das sich für gesellschaftspolitische Belange der Bürger*innen von Agogo einsetzte, organisierten sie Demonstrationen gegen land grabbing im Allgemeinen und gegen die Landvergabe an ScanFarm. In der Projektgegend von BioFuel Africa hingegen, gelang es ActionAid Ghana nicht, die Landnutzer*innen zu mobilisieren.

Eine Annahme, von der ich zu Beginn meiner Forschung ausgegangen bin, war, dass die Ausgestaltung traditioneller Herrschaftssysteme das Handeln traditioneller Autoritäten beeinflusst und sich somit auf Konflikte auswirkt. Meine empirischen Erkenntnisse bestätigen dies nur teilweise. Der einzige Anhaltspunkt, der die Annahme stützt, ist folgender. Bezüglich der Vergabe von Land bestehen Unterschiede zwischen dem chieftaincy-System der Ashanti und demjenigen der Dagomba. Diese Unterschiede betreffen die Hierarchien, die in den beiden traditionellen Herrschaftssystemen bestehen und 
die Entscheidungshoheit unterschiedlicher traditioneller Autoritäten in der Landvergabe. Diese Unterschiede haben sich auf den Ablauf der Landvergabe und damit auch auf den Verlauf der Konflikte um Zugang zu Land ausgewirkt. In der Hierarchie des chieftaincy-System der Dagomba in der Northern Region existiert eine dem paramount chief übergeordnete traditionelle Autorität der $\mathrm{Ya} \mathrm{Naa}$ - die jede großflächige Vergabe von Land bewilligen muss. Daraus ergibt sich eine Rechenschaftspflicht des paramount chiefs gegenüber dem Ya Naa. In der Ashanti Region existiert diese so nicht. Der paramount chief in der Ashanti Region war nicht auf das Einverständnis des Asantehene, des Königs der Ashanti, angewiesen, sondern konnte die Vergabe des Lands eigenständig beschließen. Aufgrund seiner fehlenden Rechenschaftspflicht gegenüber einer höheren Instanz hatte er mehr Möglichkeiten, institutionelle Verfahrensweisen zu bestimmen und versteckte Macht auszuüben, als der Tijo Naa. Das könnte eine Erklärung für die größere Transparenz in der Landvergabe an BioFuel Africa sein. Unterschiede zwischen den traditionellen Herrschaftssystemen der Ashanti und Dagomba können sich also auf das Handeln traditioneller Autoritäten auswirken. Als ebenso relevant für ihr Handeln erachte ich jedoch die Unterschiede in den Persönlichkeiten der paramount chiefs: ihre gesellschaftliche Stellung, die Ressourcen, die ihnen zur Verfügung stehen, ihren Bildungsgrad etc. Darüber hinaus ist für ihr Handeln bezüglich der Vergabe von Land die Fähigkeit, Macht über Landnutzer*innen ausüben zu können, von Bedeutung. Diese Fähigkeit hängt wiederum damit zusammen, über welche Mechanismen Landnutzer*innen Zugang zu Land erlangen oder welche Landrechte sie einfordern können. Die Ausgestaltung des Zugangs zu Land ist jedoch nicht nur durch Unterschiede in den traditionellen Herrschaftssystemen bestimmt, sondern hängt ebenfalls mit den kontextspezifischen Formen der Landnutzung zusammen. Hier schließe ich an Amanor (2008) und Berry (2009a) an und argumentiere, dass sich Unterschiede im Zugang zu Land über die Geschichte der landwirtschaftlichen Entwicklung in der jeweiligen Gegend erklären lassen. In der Ashanti Region hat sich maßgeblich der dort verbreitete Kakao-Anbau auf die Landrechtsansprüche der Landnutzer*innen ausgewirkt. Er hat dazu geführt, dass die Kakaobäuerinnen und -bauern individuelle Landrechte geltend machen konnten, auf die der paramount chief keinen Einfluss hatte. In der Northern Region gab es diese Entwicklung nicht, sondern die Landvergabe erfolgte immer über den chief. Das führte zu einer größeren Abhängigkeit der Landnutzer*innen von traditionellen Autoritäten und verlieh dem paramount chief entsprechend mehr unsichtbare Macht. 
Trotz Unterschieden in den Herrschaftssystemen der Ashanti und Dagomba sowie teilweise unterschiedlichen Machtressourcen und Handlungsstrategien der paramount chiefs sind die übergeordneten Erkenntnisse aus beiden Fällen gleich. Die Entstehung der Konflikte ging in beiden Fällen auf die Verschiebung der Kontrolle über den Zugang zu Land zugunsten der paramount chiefs zurück. Die Verschiebung von Kontrolle über Zugang ist typisch für Kontexte, die wie land grabbing durch strukturelle Veränderungen, wie die Ausweitung der agrarindustriellen Produktionsweise, die Veränderung von Landbesitzverhältnissen oder der Transformation von Arbeitsbeziehungen gekennzeichnet sind (Dietz/Engels 2020; Peluso/Lund 2011). Für die Landnutzer*innen wirkte sich die Veränderung von Kontrolle negativ auf ihren Zugang zu Land aus. Sie hatten jedoch keine Möglichkeiten, die paramount chiefs dafür zur Rechenschaft zu ziehen. Das Problem der mangelnden Rechenschaftspflicht beschreibt auch Janine Ubink (2008a: 92). Dieses rückt die Bedeutung des Verhältnisses von staatlicher und traditioneller Herrschaft in den Mittelpunkt, die auch andere Studien hervorheben (Amanor 2008; Boone 2015; Claassens/Cousins 2008; Peters 2013a; Ubink 2008a). Die Anerkennung und Legitimierung traditioneller Herrschaft durch den Staat, die Entscheidungshoheit traditioneller Autoritäten in Landverwaltungsfragen sowie fehlende rechtliche Möglichkeiten der Landnutzer*innen, Zugang zu Land zu verteidigen oder einzufordern, tragen maßgeblich dazu bei, dass traditionelle Autoritäten so handeln können, wie sie handeln. Mit der Veränderung von Kontrolle ging des Weiteren ein Wandel in den sozialen Beziehungen rund um den Zugang zu Land einher. Um weiterhin Zugang zu Land erlangen zu können, mussten Landnutzer*innen Rechte neu verhandeln. In diesem Kontext haben in beiden Fällen klientelistische und kapitalistische Beziehungen an Bedeutung gewonnen. Unter dem Bedeutungsgewinn klientelistischer Beziehungen verstehe ich, dass durch die Landvergabe die Beziehung zu den paramount chiefs wichtiger geworden ist, um Zugang zu Land zu erlangen. Im Fall von ScanFarm war die Beziehung zum paramount chief von Vorteil, um einen Arbeitsplatz bei dem Unternehmen zu erhalten. Auch für die Bestätigung vorheriger Landrechte erwies sich die Beziehung zum paramount chief als relevant. Im Fall von BioFuel Africa war die enge Beziehung zum paramount chief ebenfalls zentral für eine Anstellung bei dem Unternehmen. Auch Informationen und der Kontakt zum Unternehmen, um Zugang zu Land zu schützen, liefen über den paramount chief. Unter dem Bedeutungsgewinn kapitalistischer Beziehungen für den Zugang zu Land verstehe ich, dass Landnutzer*innen nach der Landvergabe Zugang zu Land hauptsächlich über die 
Unternehmen und zumeist in Form von Geld - seien es Entschädigungszahlungen oder Lohn für Arbeit - erlangen können. Traditionelle Landrechte hingegen verloren an Relevanz. Sie ermöglichten Landnutzer"innen in beiden Fällen nicht oder nur sehr begrenzt, ihren Zugang zu Land aufrechtzuerhalten. Die vieldiskutierte Verhandelbarkeit und flexible Interpretation traditioneller Rechte führte im Zusammenspiel mit der sichtbaren, versteckten und unsichtbaren Macht nicht etwa zu einem größeren Handlungsspielraum der Landnutzer*innen, sondern zur Verstärkung sozialer Ungleichheiten und zur Reproduktion von Machtverhältnissen. Mit dieser Beobachtung schließe ich an Amanor (1999) und Peters (2004) an. Mit Blick auf zunehmende Konkurrenz und Konflikte um Land, die im Rahmen struktureller Veränderungen auftreten, kommen sie ebenfalls zu diesem Schluss. Mithilfe meines Analyserahmens konnte ich nicht nur zeigen, dass sich im Zusammenhang mit land grabbing der Zugang zu Land zuungunsten der vorherigen Landnutzer*innen verändert hat. Ich konnte darüber hinaus ein differenziertes Bild der unterschiedlichen Zugangsmechanismen herausarbeiten und diese in einen Zusammenhang mit der sozialen Zugehörigkeit der Landnutzer*innen setzen. So konnte ich zeigen, wie genau sich die Verluste und Gewinne in Bezug auf Zugang zu Land verteilt haben. Hinsichtlich der Erklärung von Konflikten um Zugang zu Land hat das Konzept der sichtbaren, unsichtbaren und versteckten Macht Aufschluss darüber gegeben, inwiefern Machtverhältnisse eine vermittelnde Funktion zwischen der Kontrolle des Zugangs zu Land und dem Auftreten von Konflikten spielen. Eine Stärke des Konzepts war es, auch die Bedeutung von Nicht-Handeln für die Erklärung von Konflikten zu erkennen. Über die Analyse der unterschiedlichen Machtformen konnte ich zeigen, warum Konflikte bestehen können, auch wenn sie nicht manifest werden, also in Handeln resultieren.

\subsection{Formulierung von Hypothesen}

Auf der Grundlage meiner empirischen Erkenntnisse formuliere ich drei Hypothesen zur Erklärung von Konflikten um Zugang zu Land, die im Zusammenhang mit land grabbing in traditionellen Landverwaltungssystemen auftreten. In den Hypothesen greife ich die zu Beginn dieses Buches formulierten Fragen auf. Diese lauteten: (1) Wie verändert sich Kontrolle über den Zugang zu Land im Kontext von land grabbing? (2) Wie verändern sich in diesem Zusammenhang traditionelle Herrschaftssysteme? Und (3) wie 
wirkt sich das Handeln traditioneller Autoritäten auf die Entstehung und den Verlauf von Konflikten um Zugang zu Land aus?

Hypothese 1: Im Zusammenhang mit land grabbing bewirkt das Handeln ranghoher traditioneller Autoritäten die Reproduktion von Machtverhältnissen in traditionellen Herrschaftssystemen.

Viele traditionelle Herrschaftssysteme weisen eine deutliche Stratifizierung, tief verankerte Hierarchien und Machtverhältnisse auf (Grischow 2008). Die für meine Forschung relevanten traditionellen Herrschaftssysteme in Ghana bilden diesbezüglich keine Ausnahme. In beiden Systemen kommen institutionalisierte Hierarchien in einer klaren Rangordnung traditioneller Autoritäten zum Ausdruck. An der Spitze dieser Herrschaftssysteme steht jeweils ein König - der Asantehene bei den Ashanti und der Ya Naa bei den Dagomba. Unter dem König befinden sich die paramount chiefs und unter diesen die sub-chiefs. In dieser Hierarchie ist die Stellung rangniederer Autoritäten immer damit verbunden, dass sie die jeweils höhere Autorität anerkennen und sich ihr gegenüber als loyal erweisen. Darüber hinaus genießen sie weitreichende Freiheiten in der Ausübung ihrer alläglichen Tätigkeiten. Meine Forschung belegt dies mit Blick auf die Verwaltung von Land. Subchiefs handelten diesbezüglich in beiden Fällen eigenverantwortlich und ohne Rücksprache mit dem paramount chief. In ihrem Zuständigkeitsbereich waren sie in ihren Entscheidungen über die Vergabe von Land für die Subsistenzwirtschaft vollkommen unabhängig. Damit ging einher, dass die paramount chiefs keine Kenntnisse über die Nutzung des durch die sub-chiefs verwalteten Lands hatten. Sie wussten nicht, welches Stück Land wie und durch wen genutzt wird und forderten auch keine Informationen diesbezüglich ein.

Im Zusammenhang mit der großflächigen Vergabe von Land an die Unternehmen ScanFarm und BioFuel Africa erlangten Hierarchien, die zuvor bereits in den traditionellen Herrschaftssystemen bestanden, aber keine Bedeutung für die alltägliche Landverwaltung hatten, an neuer Relevanz. Mit ihrem Auftreten als Eigentümer des Lands gegenüber den Unternehmen beanspruchten die paramount chiefs die Entscheidungshoheit über den $\mathrm{Zu}$ gang zu Land für sich. Damit forderten sie lang etablierte Normen in der gewohnheitsrechtlichen Verwaltung von Land heraus. Die Loyalität, die subchiefs ihrem paramount chief entgegenbringen (müssen), um ihre Stellung im traditionellen Herrschaftssystem nicht zu gefährden, bedeutet wiederum den paramount chief in seiner Autorität anzuerkennen. Entscheidungen, die der paramount chief trifft, können sub-chiefs nicht infrage stellen, ohne als illoyal zu gelten. Im Kontext von land grabbing verändert sich die Bedeutung 
von Zugang von Land. Die innere Logik traditioneller Herrschaftssysteme ermöglicht den paramount chiefs in diesem Zusammenhang die Kontrolle über Land, das zuvor die sub-chiefs verwalteten, zu erlangen. Durch die Zentralisierung der Kontrolle über den Zugang zu Land weiten sie ihre Macht aus und reproduzieren bestehende Hierarchien.

Hypothese 2: Im Zusammenhang mit land grabbing bewirkt das Handeln ranghoher traditioneller Autoritäten eine Zentralisierung von Kontrolle über Zugang zu Land. Damit gehen die Schwächung traditioneller Landrechte und die Verstärkung sozialer Ungleichheit einher.

Zahlreiche Beiträge, die sich mit land grabbing beschäftigen, heben die analytische Bedeutung von Kontrolle hervor (Borras/Franco/et al. 2012; Peluso/Lund 2011; Wilson 2015). Borras/Franco/et al. (2012) argumentieren, land grabbing sei im Wesentlichen control grabbing, da es sich insbesondere um die Aneignung von Kontrolle über den Zugang zu Land handele (ebd. : 850). Ebenfalls aus der Perspektive sich wandelnder Kontrollverhältnisse distanzieren sich auch Peluso und Lund (2011) von land grabbing im Sinne eines Containerbegriffs. Sie sehen land grabbing als eine Vielzahl sich wandelnder Kontexte, in denen neue Kräfte und Widersprüche entstehen, die sich auf die Kontrolle des Zugangs zu Land auswirken (ebd.: 669). Akteure können Kontrolle über den Zugang zu Land ausüben, indem sie neue Zugangsmechanismen bestimmen oder alte konsolidieren, indem sie eigene Ansprüche auf Zugang zu Land durchsetzen oder anderen Akteuren Zugang zu Land verwehren. Die Aneignung von Kontrolle kann über vielfältige Mechanismen erfolgen: über die räumlich-administrative und politische Umstrukturierung eines Gebiets und seiner Abgrenzung nach außen (Territorialisierung) (Vandergeest/Peluso 1995), über den Erwerb dokumentierter (und meistens staatlich legitimierter) Eigentums- oder Nutzungsrechte, über die Einhegung von Land durch Zäune oder Wachpersonal und über die Androhung oder Ausübung von Gewalt (Peluso/Lund 2011: 668). Die unterschiedlichen Kontrollmechanismen sind nicht unbedingt aufeinander abgestimmt oder dienen dem gleichen Ziel. Sie können sich ergänzen oder in Konkurrenz zueinander stehen (ebd.). Über welche Mechanismen sich Akteure im Zusammenhang mit land grabbing Kontrolle über Land aneignen (können), ist unter anderem durch die rechtlichen Rahmenbedingungen, die vorherige Nutzung des Lands und den Zweck der Aneignung bestimmt.

Durch meine Forschung in zwei unterschiedlichen traditionellen Landverwaltungssystemen in Ghana konnte ich zeigen, dass im Vorfeld von land grabbing die Kontrolle über den Zugang zu Land auf mehrere Akteure ver- 
teilt war: auf indigene Landnutzer*innen und auf ranghohe sowie rangniedere traditionelle Autoritäten. Sie alle ermöglichten wiederum einer Vielzahl anderer Akteur*innen Zugang zu Land, indem sie ihnen Landrechte übertrugen, die Nutzung des Lands gewährten oder Vereinbarungen wie Pacht- oder sharecropping-Verträge mit ihnen schlossen. Im Zusammenhang mit land grabbing veränderten sich die Kontrollverhältnisse. Aufgrund des Handelns einzelner ranghoher traditioneller Autoritäten - in beiden Fällen paramount chiefs verloren indigenen Landnutzer*innen und rangniedere traditionelle Autoritäten die Kontrolle über den Zugang zu Land. Über die Einflussnahme auf institutionelle Verfahrensweisen gelang es den paramount chiefs, sich die Kontrolle über den Zugang zu Land anzueignen. Es kam zu einer Zentralisierung von Kontrolle zu Gunsten der paramount chiefs, die schließlich die Kontrolle über den Zugang zu Land an die Unternehmen übertrugen. Möglich war dies aufgrund der fehlenden Rechenschaftspflicht der paramount chiefs gegenüber sub-chiefs, Landnutzer*innen sowie staatlichen Akteuren, die laut nationaler Gesetzgebung in die großflächige Vergabe von Land für die agrar-industrielle Nutzung mit einbezogen werden sollten. Zudem boten sich Landnutzer*innen und sub-chiefs keine Handlungsoptionen, um ihre Rechte an Land einzufordern und durchzusetzen.

Durch die Veränderung der Kontrollverhältnisse fanden selbst Rechte, die zuvor als permanent galten, keine oder nur unzulängliche Berücksichtigung. Die Lebensbedingungen aller Akteure, denen die Kontrolle über den Zugang $\mathrm{zu}$ Land entzogen wurde oder die ihren Zugang zu Land verloren, verschlechterten sich im Zuge der Landvergabe.

Jedoch waren nicht alle Landnutzer*innen gleichermaßen von den Auswirkungen der veränderten Kontrollverhältnisse betroffen. Vielmehr belegen die erhobenen Daten eine Verstärkung bereits zuvor bestehender sozialer Ungleichheitsverhältnisse. Auch wenn die Landnutzer*innen und sub-chiefs in beiden untersuchten Fällen die Verlierer*innen der Landvergabe waren, gelang es manchen von ihnen, Entschädigungszahlungen oder die Bereitstellung alternativer Felder durch die Unternehmen für den Verlust ihres Lands einzufordern oder ein Einkommen als Arbeiter*in für das Unternehmen zu erhalten. Zu diesen Landnutzer*innen zählten jedoch nicht diejenigen, deren Zugang zu Land bereits vor der Landvergabe unsicher war wie Migrant*innen und Landnutzer*innen mit kollektiven Zugangsrechten. Sie trafen die Auswirkungen der veränderten Kontrollverhältnisse am stärksten.

In traditionellen Landverwaltungssystemen hängt die Kontrolle über den Zugang zu Land also von mehr als dem Erwerb schriftlich dokumentierter 
Landnutzungsrechte $\mathrm{ab}$. Um Kontrollverhältnisse zu verändern bedarf es vielmehr einer Neuinterpretation bestehender Regeln und Verfahrensweisen sowie ihrer Anerkennung als legitim.

Hypothese 3: Ranghohe traditionelle Autoritäten üben durch ihr Handeln im Zusammenhang mit land grabbing auf unterschiedlichen Ebenen Macht gegenüber den Landnutzer*innen aus. Dadurch beeinflussen sie maßgeblich den Verlauf von Konflikten um Zugang zu Land.

Macht - verstanden als die Fähigkeit, Kontrolle über Zugang zu Land ausüben zu können - ist von zentraler Bedeutung für die Analyse von Konflikten, die im Zusammenhang mit land grabbing auftreten. In Abhängigkeit des Kontexts, in dem Konflikte auftreten, üben Akteure unterschiedliche Formen von Macht aus, mittels derer sie den Zugang zu Land kontrollieren. Die unterschiedlichen Formen von Macht strukturieren Konflikte auf unterschiedliche Weise. Sie wirken sich darauf aus, ob Konflikte latent verlaufen oder manifest werden, ob es zu Widerstand kommt oder nicht, und auf welche Konfliktregelungsformen sich die Akteure verständigen (können).

Typisch für traditionelle Landverwaltungssysteme ist die Informalität ihrer Institutionen. Regeln und Verfahrensweisen sind nicht verschriftlicht und treten nicht über »offizielle«, staatliche Wege in Kraft. Sie erlangen ihre Geltung durch Autolizenzierung und hängen in ihrem Fortbestand von ihrer faktischen Anerkennung ab (Helmke/Levitsky 2004: 727).

Die Erkenntnisse aus meiner Forschung zeigen, dass ranghohe traditionelle Autoritäten auf unterschiedlichen Ebenen Macht gegenüber den Landnutzer*innen ausgeübt haben, um den Zugang zu Land zu kontrollieren. Als besonders relevant haben sich versteckte und unsichtbare Formen der Macht erwiesen. Diese haben sich in ihrem Zusammenspiel maßgeblich auf den Verlauf von Konflikten um Zugang ausgewirkt. Über ihre Einflussnahme auf institutionelle Verfahrensweisen, deren Neuinterpretation oder die Verfügungsgewalt über Informationen konnten ranghohe traditionelle Autoritäten de facto alleinige Kontrolle über den Zugang zu Land erlangen und diese im Rahmen der Landvergabe - entgegen den Interessen der Landnutzer*innen - an die Unternehmen übertragen. Unsichtbare Macht trägt ihrerseits zur Festigung versteckter Macht bei: die Anerkennung traditioneller Autoritäten durch die ihnen untergebenen Mitglieder der Gemeinschaft legitimiert ihr Handeln und verhindert Widerstand. Gegner*innen, auf die traditionelle Autoritäten unsichtbare Macht ausüben (können), stellen das Handeln traditioneller Autoritäten weder offen in Frage, noch fordern sie es heraus oder fechten es an. Für Konflikte bedeutet dies, dass sie eher latent 
verlaufen. Dank ihrer unsichtbaren Macht können traditionelle Autoritäten entgegen der Interessen ihrer Gemeinschaft handeln, ohne dass ein manifester Konflikt entsteht. Eine starke Legitimität traditioneller Autoritäten - die unter anderem mit ihrer Anerkennung aufgrund von Abhängigkeitsverhältnissen zusammenhängt - bewirkt wiederum, dass das Handeln traditioneller Autoritäten nicht infrage gestellt wird. Das bedeutet, dass mangelnde unsichtbare Macht offenen Widerstand und damit eine Manifestierung von Konflikten begünstigen kann. In den untersuchten Konflikten übten diejenigen Konfliktakteure offenen Widerstandstand aus, die ihre Landrechte nicht über traditionelle Autoritäten erlangt hatten, für deren alltägliches Leben die Beziehungen $\mathrm{zu}$ traditionellen Autoritäten nicht relevant waren und für die eine Nicht-Einhaltung traditioneller Normen und Regeln keine negativen Auswirkungen hatte. Im Fall von ScanFarm waren dies Landnutzer*innen mit formalisierten traditionellen Rechten und solche, die geschlossen als traditionelle Gemeinschaft auftraten und sich gegenseitig stärkten, indem sie sich ihre bestehende Rechte gegenseitig bestätigten. Im Fall von BioFuel Africa waren es Landwirt*innen, die nicht in der Gegend lebten, sondern nur Land dort bestellten. Zugang zu diesem Land hatten sie über rangniedere traditionelle Autoritäten erhalten. Einen Anspruch auf Entschädigung hatten sie jedoch nicht als sie den Zugang zu Land aufgrund des Vorhabens von BioFuel Africa verloren. Im Gegensatz zu den Bewohner*innen der Dörfer in der Projektgegend, gab es für diese Landwirt*innen daher keine Notwendigkeit die Autorität des paramount chiefs anzuerkennen, indem sie seine Entscheidung mittrugen. Darüber hinaus waren sie nicht von einer guten Beziehung zu ihm abhängig, was ihnen die Möglichkeit gab, die Legitimität seines Handelns infrage zu stellen. Diese Landnutzer*innen positionierten sich am deutlichsten gegen die Landvergabe und protestierten gegen den Verlust ihres Zugangs zu Land.

\subsection{Reichweite der Hypothesen}

Die von mir entwickelten Hypothesen bieten Erklärungsansätze bezüglich des Zusammenhangs zwischen dem Handeln traditioneller Autoritäten und Konflikten um Zugang zu Land, die im Kontext von land grabbing auftreten. Die Erkenntnisse aus der kollektiven Fallstudie sind, dass traditionelle Autoritäten über ihr Handeln im Zusammenhang mit land grabbing zu einer Zentralisierung von Kontrolle über den Zugang zu Land, einer Reproduktion von 
Machtverhältnissen in traditionellen Landverwaltungssystemen, der Schwächung traditioneller Landrechte und der Verstärkung sozialer Ungleichheit beigetragen haben. Des Weiteren konnte ich zeigen, dass sich die Ausübung unterschiedlicher Machtformen maßgeblich auf die Entstehung und den Verlauf von Konflikten auswirkt.

Hinsichtlich der formulierten Hypothesen erhebe ich keinen Anspruch auf Allgemeingültigkeit. Ich argumentiere jedoch, dass die Erkenntnisse aufgrund des Designs meiner Forschung auch über die untersuchten Fälle hinaus relevant sind. Inwiefern sie auch für andere Kontexte plausibel sind und welche Grenzen die Auswahl der Fälle bezüglich der Generalisierbarkeit der Erkenntnisse setzt, diskutiere ich im folgenden Abschnitt.

In meinem vergleichenden Forschungsdesign habe ich eine diversecase-Strategie verfolgt. Diese zeichnet sich dadurch aus, dass sich die Fälle in zentralen Aspekten des theoriegeleiteten Erkenntnisinteresses unterscheiden. Darüber hinaus zeichnen sie sich durch eine gute Vergleichbarkeit der Kontextfaktoren aus (Gerring 2007: 97). Der zentrale Unterschied zwischen den beiden erforschten Fällen bestand in den traditionellen Herrschaftssystemen, da meine Vorannahme war, dass ihre Ausgestaltung das Handeln der Akteure beeinflusst. Die diverse-case-Strategie verbessert die Möglichkeit, Erkenntnisse und Schlussfolgerungen auf andere Kontexte übertragen zu können, insofern intervenierende Kontextfaktoren möglichst gleich gehalten werden (Gerring 2007: 97f.). Dementsprechend habe ich darauf geachtet, dass meine Fälle, im Vergleich mit anderen in der Literatur beschriebenen Konflikten, die im Zusammenhang mit land grabbing auftreten, typisch sind. Für die Auswahl habe ich folgende Kriterien berücksichtigt: den Zeitpunkt der Landvergabe, das Ausmaß der gepachteten Fläche, die involvierten Akteure, den Zweck der Umnutzung und die vorherige Form der Landnutzung. Der Zeitpunkt beider Landvergaben, in dessen Zusammenhang die von mir untersuchten Konfliktfälle auftraten, lässt sich der gleichen konjunkturellen Phase zuordnen. Diese Phase zeichnet sich durch multiple globale Krisen seit Mitte der 2000er Jahre aus, in dessen Kontext es zu einem weltweiten Anstieg von land grabbing kam. Diesem Zeitraum lassen sich meine Fälle zuordnen. Ebenso wie in vielen anderen Kontexten strebten in den von mir ausgewählten Fällen internationale Investoren die Pacht mehrerer Tausend Hektar Land für den Anbau von flex crops an, setzten ihre Vorhaben schließlich jedoch auf weitaus kleineren Flächen um (Nolte et al. 2016). Ebenfalls typisch ist ein Wandel von der kleinbäuerlichen, subsistenzorientierten Produktion 
hin zur agrarindustriellen, oftmals exportorientierten, Produktion. All diese Kontextfaktoren ermöglichen eine gute Vergleichbarkeit mit anderen Fällen.

Darüber hinaus existieren jedoch weitere intervenierende Faktoren, die sich auf das Handeln traditioneller Autoritäten auswirken und für die Erklärung von Konflikten berücksichtigt werden müssen. Diese setzen der Reichweite der Hypothesen Grenzen. Die Übertragbarkeit meiner Forschungserkenntnisse auf Konflikte um Zugang zu Land, die in anderen Länderkontexten mit traditionellen Landverwaltungssystemen auftreten, hängt, wie ich im Folgenden ausführe, von zwei Faktoren ab: dem Verhältnis von traditioneller und staatlicher Herrschaft sowie dem Verhältnis von traditionellen Autoritäten und den Mitgliedern ihrer Gemeinschaft. Traditionelle Herrschaftssysteme existieren in vielen Ländern Subsahara-Afrikas und sind auf lokaler Ebene von zentraler Bedeutung für das alltägliche Leben. In ihrem Verhältnis zu staatlicher Herrschaft weisen traditionelle Herrschaftssysteme in unterschiedlichen Staaten jedoch deutliche Unterschiede auf, insbesondere hinsichtlich ihres rechtlichen Status, ihrer Rolle in der nationalen Politik, ihrer rechtlichen, administrativen und sozialen Stellung in der Gemeinschaft sowie ihrer Bedeutung in der Landverwaltung (Honig 2017; Logan 2013; Ubink 2008c). Der Einfluss unterschiedlicher Regierungsformen in der Landverwaltung, wirkt sich wiederum auf Konflikte um Land aus (Boone 2013, 2014). In der Analyse von Konflikten um Zugang zu Land muss daher das Verhältnis von traditioneller und staatlicher Herrschaft sowie das Verhältnis von traditionellen Autoritäten und den Mitgliedern ihrer Gemeinschaft je nach nationalem Kontext immer wieder neu analysiert und berücksichtigt werden. Folgende Fragen sind diesbezüglich zentral: Sind traditionelle Autoritäten durch den Staat anerkannt? Erlaubt die rechtliche Grundlage es der Regierung, Einfluss auf die Auswahl traditioneller Autoritäten zu nehmen? Können traditionelle Autoritäten gleichzeitig politische Ämter bekleiden oder Regierungsaufgaben ausführen? Stehen traditionelle Autoritäten auf der Gehaltsliste des Staats? Sind traditionelle Autoritäten in ihrem Handeln unabhängig vom Staat oder werden sie durch ihn überwacht? In welchem Verhältnis stehen traditionelle Autoritäten zu gewählten Regierungsvertreter"innen? Können traditionelle Autoritäten sich aktiv an der Parteipolitik beteiligen? Welche Aufgaben fallen in den Zuständigkeitsbereich traditioneller Autoritäten? Wie üben sie diese aus? Inwiefern bietet das traditionelle Herrschaftssystem funktionierende Kontrollmechanismen, um die Aufgaben und das Handeln traditioneller Autoritäten zu überwachen und zu regulieren? Inwieweit sind traditionelle Strukturen, Normen und Werte gesellschaftlich anerkannt? Wie 
beliebt und legitim sind traditionelle Autoritäten in ihrem Zuständigkeitsbereich? Ist traditionelle Herrschaft Gegenstand öffentlicher kritischer Diskussionen? (Ubink 2008c: 18).

In Ghana sind traditionelle Autoritäten rechtlich durch die staatliche Verfassung anerkannt, traditionelle und staatliche Herrschaft sind strikt getrennt, die Bekleidung politischer Ämter durch traditionelle Autoritäten ist nicht gestattet und traditionelle Autoritäten erhalten keinen Lohn durch den Staat. Traditionelle Autoritäten sind somit in ihrem Handeln unabhängig von staatlichen Akteuren und werden nicht durch sie überwacht oder reglementiert. Dies trifft insbesondere auf die Landverwaltung zu. Traditionelle Strukturen, Normen und Werte genießen zudem eine große gesellschaftliche Akzeptanz. Mit Blick auf das Verhältnis von staatlicher und traditioneller Herrschaft sowie von traditionellen Autoritäten und den Mitgliedern ihrer Gemeinschaft machen diese Faktoren Ghana zu einem extremem Fall (extreme case) (Seawright/Gerring 2008: 301). Ein solcher zeichnet sich dadurch aus, dass zentrale analytische Dimensionen besonders stark oder schwach ausgeprägt sind. Extreme Fälle eignen sich damit gut, um eine größtmögliche Informationsmenge zu wenig erforschten Phänomen zu erlangen (Flyvbjerg 2011: 16). Für die Reichweite der Hypothesen bedeutet dies, dass sie sich insbesondere auf Kontexte übertragen lassen, die ähnliche Merkmale wie Ghana aufweisen: ein staatlich anerkanntes traditionelles Herrschaftssystem, in dem staatliche Akteure traditionelle Autoritäten nicht in ihrem Handeln überwachen oder reglementieren, sondern ihnen - insbesondere auch in der Landverwaltung - freie Hand lassen, und in dem traditionelle Autoritäten eine große gesellschaftliche Akzeptanz genießen. Darüber hinaus bieten die Erkenntnisse Anknüpfungspunkte für weitere Forschung zu Konflikten um Zugang zu Land in Kontexten, in denen traditionelle Landverwaltungssysteme existieren, die sich jedoch bezüglich zentraler analytischer Dimensionen von meinen Fällen unterscheiden. Interessant könnten Länderkontexte sein, in denen tradtionelle Autoritäten Ämter in der nationalen Politik bekleiden oder in denen staatliches Recht traditionelle Herrschaftssysteme nicht anerkennt. Der Vergleich mit solchen Fällen bietet die Möglichkeit, die Erkenntnisse zu überprüfen, zu ergänzen und auszudifferenzieren. 


\subsection{Beitrag zur gegenwärtigen Forschung}

Mit diesem Buch leiste ich insbesondere zu drei Forschungsfeldern einen Beitrag. Erstens ergänze ich die Forschung zu land grabbing und Konflikten in traditionellen Landverwaltungssystemen um den Fokus auf das Handeln traditioneller Autoritäten und dessen Implikationen für die Entstehung und den Verlauf von Konflikten. Zweitens liefert meine Forschung Erkenntnisse, die für die Auseinandersetzung um die Zukunft der rechtlichen Ausgestaltung kommunaler, indigener und (neo-)traditioneller Landverwaltungssysteme relevant sind. Drittens trage ich zu einer kritischen Diskussion über traditionelle Konfliktbearbeitung bei.

\section{Land grabbing, Konflikte und das Handeln traditioneller Autoritäten}

Die wissenschaftliche Literatur zu land grabbing und Konflikten, die damit einhergehen, ist in den letzten Jahren angewachsen. Frühe Arbeiten argumentierten in ihrer Erklärung, wie und warum Konflikte im Zusammenhang mit land grabbing auftreten, meist linear. Sie stellten einen Kausalzusammenhang zwischen globalen Krisenphänomenen, ihren vermeintlichen Auswirkungen wie großflächigem Landnutzungswandel oder der Verknappung von Land und lokalen Konflikten her (Dietz/Engels 2020). Konflikte fassten sie als logische Konsequenz des Verlusts der Lebensgrundlagen lokaler Landnutzer*innen. Diese, in der Regel als homogene marginalisierte Gruppe konzipierten Akteure, leisteten demnach aufgrund von Vertreibung, Umweltverschmutzung oder der Zerstörung von Ökosystemen Widerstand, durch den Konflikte zum Ausdruck kamen (Le Billon 2015; Martinez-Alier 2002; Martinez-Alier et al. 2016). Jüngere Arbeiten zeichnen ein differenzierteres Bild von Konflikten im Kontext von land grabbing. In Hinblick auf den Zusammenhang zwischen globalem Wandel und lokalen Konflikten um Land berücksichtigen sie in ihrer Analyse eine Vielzahl an Einflussfaktoren, die auf das Handeln von Konfliktakteuren wirken. Zu diesen zählen die Machtposition der Akteure, ihre Differenzierung entlang sozialer Kategorien wie Klasse, Geschlecht, Alter oder Herkunft, politisch-institutionelle Kontextbedingungen, das Handeln staatlicher Akteure und dominante Erzählungen, wie Entwicklungsdiskurse, die land grabbing legitimieren (Borras/Franco 2013; Dietz/Engels 2018; Engels 2021b; Hall et al. 2015). An diese Beiträge knüpfe ich an. Anders als zu Beginn meiner Forschung widmen sich mittlerweile Wissenschaftler*innen dem Thema land grabbing und Konflikte auch ver- 
mehrt mit Blick auf traditionelle Landrechtssysteme (Dell'Angelo et al. 2017), und einige Beiträge befassen sich mit der Rolle traditioneller Autoritäten im Kontext von land grabbing, jedoch ohne auf Konflikte einzugehen (Ahmed et al. 2018; Capps 2018; Honig 2017; Lanz et al. 2018; Yaro 2013). Meine Forschung leistet mit ihrem Fokus auf das Handeln traditioneller Autoritäten und dessen Bedeutung für die Entstehung und den Verlauf von Konflikten einen innovativen Beitrag zur bestehenden Literatur und ergänzt sie um umfassendes empirisches Fallwissen. Meine Forschungserkenntnisse tragen insbesondere zur Auseinandersetzung um die Kontingenz von Konflikten bei (Dietz/Engels 2020). In dieser geht es darum, dass unter ähnlichen Gegebenheiten Konflikte entstehen können oder nicht, weshalb immer wieder erneut ergründet werden muss, warum sie entstehen und wie sie auf soziale Strukturen zurückwirken (ebd.). Über die Analyse des Handels traditioneller Autoritäten konnte ich diesbezüglich zeigen, dass und warum Konflikte auch bestehen (können), ohne notwendigerweise in Handeln $\mathrm{zu}$ resultieren. Insbesondere die Berücksichtigung der unterschiedlichen Machtformen in der Analyse des Handelns war für die Entwicklung von Erklärungsansätzen aufschlussreich. Darüber hinaus zeigen meine Erkenntnisse, wie sich unterschiedliche Formen von Macht beeinflussen können, ob sich Konflikte verschärfen, manifestieren, in Widerstand resultieren oder welche Themen ins Zentrum der Konflikte rücken.

\section{Traditionelle Landverwaltungssysteme und ihre rechtliche Ausgestaltung}

Mit den Erkenntnissen aus meiner Forschung leiste ich des Weiteren einen Beitrag zur Auseinandersetzung um die Zukunft der rechtlichen Ausgestaltung kommunaler, indigener und (neo-)traditioneller Landverwaltungssysteme. Ein zentraler Diskussionspunkt in dieser Auseinandersetzung ist die Formalisierung von Landrechten durch Titulierungsprogramme sowie durch die individuelle oder kollektive Registrierung von Farm- und Weideland (Sjaastad/Cousins 2009). Die Ziele, die über die Formalisierung von Landrechten verfolgt werden, unterscheiden sich ebenso sehr, wie die unterschiedlichen Befürworter*innen dieser Strategie. Globale Finanz-, Wirtschafts- und Entwicklungsakteure sehen in der Formalisierung von Landrechten den Vorteil, auf diese Weise individualisierte Wirtschaftsgüter zu schaffen, die auf dem globalen Markt gehandelt werden können. Als solche trügen sie zu wirtschaftlichem Wachstum und Entwicklung bei, indem sie denjenigen zugänglich ge- 
macht würden, die sie vermeintlich am produktivsten nutzen könnten. Den ursprünglichen Inhaber*innen der formalisierten Rechte ermögliche der Verkauf von Land einen finanziellen Gewinn. Für Verfechter*innen traditioneller Landrechte wie nationalen und internationalen zivilgesellschaftlichen Organisationen, Netzwerken und Agrarbewegungen stellt die Formalisierung von Landrechten einen Mechanismus dar, der Landnutzer*innen vor dem Verlust des Lands an den Staat oder (globalen) Markt schütze. Das rechtliche empowerment, das Landnutzer*innen durch die Formalisierung von Landrechten erführen, gebe ihnen die Möglichkeit, ihre Landrechte selbstbestimmt zu verteidigen. Menschenrechtsverteidiger*innen setzen sich schließlich für die Formalisierung von Landrechten ein, um indigenen, in der Regel marginalisierten Gruppen, die mit Enteignung und Vertreibung konfrontiert sind, Handlungsfähigkeit zu verleihen (Boone 2019). Die drei Positionen zeigen, dass mit der gleichen Strategie unterschiedliche und teilweise widersprüchliche Ziele verfolgt werden. In die Politikberatung fließen alle drei Positionen ein, jedoch mit fehlender Trennschärfe, so dass die Formalisierung von Landrechten als Patentlösung für die unterschiedlichen Herausforderungen gesehen wird. Ein Beispiel für die Vermischung der drei Positionen ist die Arbeit von Hernando de Soto (2000), die insbesondere von internationalen Geberorganisationen wie der Weltbank großen Zuspruch erhalten hat und maßgeblichen Einfluss auf die internationale Entwicklungspolitik hatte. De Soto argumentierte, die Formalisierung von Landrechten stärke insbesondere arme Landnutzer*innen, indem sie ihnen Zugang zum Markt ermögliche, woraus sich ein wirtschaftlicher Nutzen für sie ergebe. Ein grundlegendes Problem von Formalisierungsbestrebungen, das auch Boone (2019: 384) beschreibt, ist jedoch, dass zunächst bestehende Rechte erfasst werden müssen, um im Folgenden gefestigt und abgesichert zu werden können. In traditionellen Landverwaltungssystemen, in denen unterschiedliche Landnutzer*innen parallel oder zu unterschiedlichen Zeiten Anspruch auf die Nutzung des gleichen Stück Lands haben (können), geht damit eine Umverteilung und Festschreibung von Rechten einher, die es zuvor nicht gab. Aufgrund sozialer Ungleichheitsverhältnisse bringt diese Umverteilung und Festschreibung notwendigerweise Gewinner*innen und Verlierer*innen hervor (Peters 2004).

Über meine Forschung konnte ich zeigen, dass sich im Kontext der Landvergabe an ScanFarm und BioFuel Africa formalisierte sowie nicht-formalisierte traditionelle Rechte als hinfällig erwiesen. Diese Entwicklung fand statt, obwohl Ghana zu den Ländern zählt, in denen traditionelle Landrechte besonders umfassend durch staatliches Recht gesichert sind (Alden Wily 
2017: 13). Der vermeintliche Schutz traditioneller Landrechte in Ghana beschränkt sich im Wesentlichen auf die staatliche Anerkennung traditioneller Herrschaft und ihrer Zuständigkeit in der Landverwaltung. Traditionelle Landrechte sind als Gemeinschaftsrechte gesichert. Institutionalisierter Schutz für die Landrechte der einzelnen Landnutzer*innen besteht nicht und auch Sanktionsmöglichkeiten für die unrechtmäßige Vergabe von Land durch traditionelle Autoritäten fehlen. Im Kontext von land grabbing hat die starke traditionelle Herrschaft somit nicht zum Schutz von Rechten beigetragen, sondern das Gegenteil bewirkt, da traditionelle Autoritäten ihrer Gemeinschaft gegenüber nicht zu Rechenschaft verpflichtet sind. Die staatliche Anerkennung gemeinschaftlicher Rechte an Land war nicht von gemeinschaftlichem Nutzen, da aufgrund von Ungleichheitsverhältnissen nicht alle Landnutzer*innen gleichermaßen die Möglichkeit hatten, ihre Rechte einzufordern oder zu verteidigen. Diese Erkenntnis ist auch mit Blick auf Bestrebungen der Formalisierung von Zugang zu Land relevant. Sie verdeutlicht die Problematik der Verschärfung sozialer Ungleichheitsverhältnisse durch die Festschreibung individueller Rechte, die in rechtspluralistischen Landverwaltungssystemen in der Regel Gewinner*innen und Verlierer*innen hervorbringt.

\section{Traditionelle Konfliktbearbeitung aus kritischer Perspektive}

Schließlich erweitere ich mit diesem Buch die Forschung $\mathrm{zu}$ traditioneller Konfliktbearbeitung um eine kritische Perspektive. Im Kontext zunehmender innerstaatlicher Konflikte in den 1990er Jahren und einem damit einhergehenden erhöhten Bedarf an neuen Konfliktlösungsansätzen stieg das Interesse an traditionellen Autoritäten als Mediator*innen oder Schlichter*innen in Konflikten. Seit Beginn der 200oer Jahre widmen sich zahlreiche Autor*innen den Möglichkeiten, Herausforderungen und Grenzen traditioneller Konfliktbearbeitung und auch der Rolle, die traditionelle Autoritäten auf diesem Feld spielen. Als Vorteile traditioneller Konfliktbearbeitung gelten insbesondere die einfache Zugänglichkeit traditioneller Institutionen und die geringen Kosten im Vergleich zu staatlicher Konfliktlösung (Mutisi/SansculotteGreenidge 2012). Einige Autor*innen argumentieren, traditionelle Autoritäten, welche die Kolonialzeit überdauert hätten, verfügten über eine besondere Legitimität innerhalb ihrer Gemeinschaft, da sie während der Kolonialzeit zentrale Figuren im anti-kolonialen Widerstand gewesen seien, als Sinnbilder kultureller Identität gesehen würden oder ihre gesellschaftliche Anerken- 
nung tief in der jeweiligen Kultur verankert sei. Ihre gegenwärtige soziale und politische Legitimität leite sich auch heute noch aus prä-kolonialer Zeit ab (De Juan 2017; Ray/Reddy 2003). Dieses Argument geht von dem Bestehen einer kohärenten Gemeinschaft aus, in der traditionelle Autoritäten für sozialen Zusammenhalt, Frieden und Stabilität stehen. Macht- und Ungleichheitsverhältnissen sowie deren Implikationen für die Konfliktbearbeitung bleiben hierbei unberücksichtigt.

Einige Autor*innen aus der friedenspolitischen Praxis und Forschung integrieren in ihren Beiträgen eine stärker kritische Perspektive. Hinsichtlich der Nachteile traditioneller Konfliktbearbeitung verweist Volker Boege (2006: 17) darauf, dass traditionelle Herrschaftssysteme eine Tendenz zur Aufrechterhaltung des Status quo hätten. In der traditionellen Konfliktbearbeitung bestehe daher die Gefahr der Reproduktion der "guten alten Ordnung«, was sich als nachteilig für die schwächeren Mitglieder der Gemeinschaft erweisen könne. Auf die zentrale Bedeutung von Machtungleichheitsverhältnissen in der traditionellen Konfliktbearbeitung verweist auch Susanne Buckley-Zistel (2005) in ihrer Studie zu gacaca-Gerichten in Ruanda. An diese Erkenntnisse schließe ich mit meiner Forschung an. Rukuni et al. (2015) sind in ihrer Studie zu traditioneller Konfliktbearbeitung in Simbabwe darüber hinaus zu dem Schluss gekommen, dass traditionellen Autoritäten in der Konfliktbearbeitung oftmals die Unparteilichkeit fehle, insbesondere wenn es sich um Fälle handelt, die ihre eigenen Interessen berühren. Sie stellen zudem fest, dass es traditionellen Autoritäten in der Konfliktbearbeitung oftmals an partizipatorischen Fähigkeiten, wie sie die Mediation voraussetzt, fehlt. Diesbezüglich hinterfragen sie kritisch, inwiefern die auf Erbschaft beruhende Position traditioneller Autoritäten sie für die Konfliktbearbeitung qualifizieren könne. Als problematisch sehen sie zudem fehlendes Vertrauen der Gemeinschaft, in Fällen, in denen traditionelle Autoritäten als offensichtlich korrupt oder parteiisch auftreten.

In der bestehenden Forschung zu diesem Themenfeld fehlt bisher die Erforschung traditioneller Konfliktlösung in Konflikten, in denen traditionelle Autoritäten selbst involviert sind. Meine Forschungserkenntnisse tragen zur Schließung dieser Lücke bei und ergänzen die gegenwärtigen Beiträge zudem um eine handlungszentrierte, machtkritische Analyse der Rolle traditioneller Autoritäten in der Konfliktlösung. Für die Forschung zu traditioneller Konfliktbearbeitung leitet sich aus meiner Studie eine Relevanz ab, den Konfliktlösungsprozess genauer in den Blick zu nehmen und zu erforschen, inwiefern Entscheidungen partizipatorisch getroffen werden, welche Lösungsan- 
sätze überhaupt zu Diskussion gestellt werden, welche von vornherein ausgeschlossen werden und inwiefern Konflikte dadurch tatsächlich oder nur vermeintlich gelöst sind. 


\section{Literaturverzeichnis}

ActionAid/FoodSPAN (2010): Impact of Biofuel Production on Poor Communities in Ghana. Policy Brief. Accra: ActionAid Ghana.

ActionAid Ghana (2011): Annual Report 2010. Accra: ActionAid Ghana.

Adjei, Prince Osei-Wusu/Busia, Abrefa Kwaku/Bob-Milliar, George Meyiri (2017): Democratic decentralization and disempowerment of traditional authorities under Ghana's local governance and development system: a spatio-temporal review. Journal of Political Power, 10(3), 303-325.

Agyekum, Kofi (2006): The Sociolinguistic of Akan Personal Names. Nordic Journal of African Studies, 15(2), 206-235.

Ahmed, Abubakari/Kuusaana, Elias Danyi/Gasparatos, Alexandros (2018): The role of chiefs in large-scale land acquisitions for jatropha production in Ghana: insights from agrarian political economy. Land Use Policy, 75, 570582.

Ahorsu, Ken (2014): A Poststructuralist Approach to the Dagbon Chieftaincy Crisis in Northern Ghana. African Conflict and Peacebuilding Review, 4(1), 95119.

Akram-Lodhi, A. Haroon (2012): Contextualising land grabbing: contemporary land deals, the global subsistence crisis and the world food system. Canadian Journal of Development Studies/Revue canadienne d'études du développement, 33(2), 119-142.

Alden Wily, Liz (2012): Looking back to see forward: the legal niceties of land theft in land rushes. Journal of Peasant Studies, 39(3-4), 751-775.

Alden Wily, Liz (2017): Customary tenure: remaking property for the 21st century. In: Graziadei, Michele \& Smith, Lionel (Hg.), Comparative Property Law: Global Perspectives. Cheltenham: Edward Elgar, 458-478.

Amanor, Kojo S. (1999): Global Restructuring and land rights in Ghana. Forest food chains, timber, and rural livelihoods. Research Report Nr. 108. Uppsala: Nordiska Afrikainstitutet. 
Amanor, Kojo S. (2001): Land, labour and the family in southern Ghana. A Critique of Land Policy under Neo-Liberalisation. Research Report Nr. 116. Uppsala: Nordiska Afrikainstitutet.

Amanor, Kojo S. (2005): Night Harvesters, Forest Hoods and Saboteurs: Struggles over Land and Expropriation in Ghana. In: Moyo, Sam \& Yeros, Paris (Hg.), Reclaiming the Land: The Resurgence of Rural Movements in Africa, Asia and Latin America. London, New York: Zed Books, 102-117.

Amanor, Kojo S. (2008): The changing face of customary tenure. In: Ubink, Janine M. \& Amanor, Kojo S. (Hg.), Contesting land and custom in Ghana: State, chief and the citizen. Leiden: Leiden University Press, 55-79.

Amanor, Kojo S. (2009): Securing land rights in Ghana. In: Ubink, Janine M., Hoekema, André J., \& Assies, Willem J. (Hg.), Legalising Land Rights: Local Practices, State Responses and Tenure Security in Africa, Asia and Latin America. Leiden: Leiden University Press, 97-131.

Amanor, Kojo S. (2012): Global resource grabs, agribusiness concentration and the smallholder: two West African case studies. The Journal of Peasant Studies, 39(3-4), 731-749.

Annan, Nancy/Kabran, Emmanuela/Onoma, Ato (2013): Ghana: The Land Question in El Dorado. ECOWAS Peace and Security Report Issue 6. Dakar: Institue for Security Studies.

Anthias, Floya (2006): Belongings in a Globalising and Unequal World: Rethinking Translocations. In: Yuval-Davis, Nira, Kannabiran, Kalpana, \& Vieten, Ulrike (Hg.), The Situated Politics of Belonging. London: SAGE Publications, 17-31.

Arhin-Sam, Kwaku (2019): Return migration, reintegration and sense of belonging: the case of skilled Ghanaian returnees. Baden-Baden: Nomos.

Aryeetey, Ernest (2007): The politics of land tenure reform in Ghana: from the Crown Lands Bills to the Land Administration Project. Legon: Institute of Statistical, Social \& Economic Research, University of Ghana.

Bachrach, Peter/Baratz, Morton S. (1962): Two Faces of Power. American Political Science Review, 56(4), 947-952.

Bachrach, Peter/Baratz, Morton S. (1970): Power and Poverty. Theory and Practice. New York: Oxford University Press.

Baldwin, Kate (2016): The paradox of traditional chiefs in democratic Africa. New York: Cambridge University Press.

Barnes, Peter (5. Juni 1971): The Great American Land Grab. The New Republic. Abrufbar unter: http://peter-barnes.org/article/the-great-american-land -grab/. Letzter Zugriff: 14.07.2020. 
Bartels, Lara Esther/Bruns, Antje/Alba, Rossella (2018): The production of uneven access to land and water in peri-urban spaces: de facto privatisation in greater Accra. Local Environment, 1-18.

Bavinck, Maarten/Pelligrini, Lorenzo/Mostert, Erik (2014): Conflicts over natural resources in the Global South : conceptual approaches. Leiden: CRC Press/Balkema.

Behrman, Julia/Meinzen-Dick, Ruth/Quisumbing, Agnes (2012): The gender implications of large-scale land deals. The Journal of Peasant Studies, 39(1), 49-79.

Benton, Ted (1981): >Objective< Interests and the Sociology of Power. Sociology, 15(2), 161-184.

Berge, Erling/Kambewa, Daimon/Munthali, Alister/Wiig, Henrik (2014): Lineage and land reforms in Malawi: Do matrilineal and patrilineal landholding systems represent a problem for land reforms in Malawi? Land Use Policy, 41, 61-69.

Berry, Sara (1989): Social Institutions and Access to Resources. Africa, 59(1), 41-55.

Berry, Sara (1992): Hegemony on a Shoestring: Indirect Rule and Access to Agricultural Land. Africa: Journal of the International African Institute, 62(3), 327-355.

Berry, Sara (1993): No condition is permanent: The social dynamics of agrarian change in sub-Saharan Africa. Madison, Wisconsin: University of Wisconsin Press.

Berry, Sara (2002): Debating the land question in Africa. Comparative Studies in Society and History, 44(4), 638-668.

Berry, Sara (2008): Ancestral property: Land, politics and the deeds of the ancestors' in Ghana and Côte d'Ivoire. In: Amanor, Kojo \& Ubink, Janine (Hg.), Contesting land and custom in Ghana: State, chief and the citizen. Leiden: Leiden University Press, 27-53.

Berry, Sara (2009a): Building for the Future? Investment, Land Reform and the Contingencies of Ownership in Contemporary Ghana. World Development, 37(8), 1370-1378.

Berry, Sara (2009b): Property, Authority and Citizenship: Land Claims, Politics and the Dynamics of Social Division in West Africa. Development and Change, 40(1), 23-45.

Berry, Sara (2013): Questions of Ownership: Proprietorship and Control in a Changing Rural Terrain - a Case Study from Ghana. Africa: Journal of the International African Institute, 83(1), 36-56. 
Berry, Sara (2017): Struggles over Land and Authority in Africa. African Studies Review, 60(3), 105-125.

Berry, Sara (2018): Who owns the land? Social relations and conflicts over resources in Africa. GLOCON Working Paper Series Nr. 5. Berlin.

Bierschenk, Thomas (2010): States at Work in West Africa: Sedimentation, Fragmentation and Normative Double-Binds. Arbeitspapiere des Instituts für Ethnologie und Afrikastudien der Johannes Gutenberg-Universität Mainz Nr. 113. Mainz: IFEAS.

Bierschenk, Thomas/Chauveau, Jean-Pierre/Olivier de Sardan, Jean-Pierre (Hg.) (2000): Courtiers en développement: Les villages africains en quête de projets. Paris: Karthala, APAD.

Bierschenk, Thomas/Olivier de Sardan, Jean-Pierre (1997): Local Powers and a Distant State in Rural Central African Republic. The Journal of Modern African Studies, 35(3), 441-468.

Blaikie, Piers M. (1985): The political economy of soil erosion in developing countries. London, New York: Longman.

Blocher, Joseph (2006): Building on Custom: Land Tenure Policy and Economic Development in Ghana. Yale Human Rights and Development Journal, 9(1), 166-202.

Boafo-Arthur, Kwame (2003): Chieftaincy in Ghana: Challenges and Prospects in the 21st Century. African and Asian Studies, 2(2), 125-153.

Boamah, Festus (2011a): Competition between biofuel and food? Evidence from a jatropha biodiesel project in Northern Ghana. In: Matondi, Prosper Bvumiranayi, Havnevik, Kjell J., \& Atakilte, Beyene (Hg.), Biofuels, land grabbing and food security in Africa. London, New York: Zed Books, 159-175.

Boamah, Festus (2011b): The Relationship between Land Grabbing for Biofuels and Food Security, a Bane or Boon? The Food Security Implications of Jatropha Biodiesel Project in Northern Ghana. Paper präsentiert auf derInternational Conference on Global Landgrabbing, Institute of Development Studies, University of Sussex.

Boamah, Festus (2014a): How and why chiefs formalise land use in recent times: the politics of land dispossession through biofuels investments in Ghana. Review of African Political Economy, 41(141), 406-423.

Boamah, Festus (2014b): Imageries of the contested concepts »land grabbing« and »land transactions «: Implications for biofuels investments in Ghana. Geoforum, 54, 324-334. 
Boamah, Festus (2015): Biofuels and land politics: Connecting the disconnects in the debate about livelihood impacts of jatropha biofuel land deals in Ghana. Dissertation, University of Bergen, Bergen.

Boamah, Festus/Overå, Ragnhild (2016): Rethinking Livelihood Impacts of Biofuel Land Deals in Ghana. Development and Change, 47(1), 98-129.

Boege, Volker (2006): Traditional Approaches to Conflict Transformation Potentials and Limits. Berghof Handbook for Conflict Transformation. Berlin: Berghof Research Center for Constructive Conflict Management.

Bohnsack, Ralf (2009): Gruppendiskussion. In: Flick, Uwe, von Kardorff, Ernst, \& Steinke, Ines (Hg.), Qualitative Forschung: Ein Handbuch. Reinbek bei Hamburg: Rowohlt, 369-384.

Bonacker, Thorsten/Imbusch, Peter (2006): Zentrale Begriffe der Friedensund Konfliktforschung: Konflikt, Gewalt, Krieg, Frieden. In: Imbusch, Peter \& Zoll, Ralf (Hg.), Friedens- und Konfliktforschung: VS Verlag für Sozialwissenschaften, 67-142.

Boone, Catherine (2013): Land Regimes and the Structure of Politics: Patterns of land-related Conflict. Africa, 83(Special Issue 01), 188-203.

Boone, Catherine (2014): Property and Political order in Africa. Land Rights and the Structure of Politics. New York: Cambridge University Press.

Boone, Catherine (2015): Land tenure regimes and state structure in rural Africa: implications for forms of resistance to large-scale land acquisitions by outsiders. Journal of Contemporary African Studies, 33(2), 171-190.

Boone, Catherine (2019): Legal Empowerment of the Poor through Property Rights Reform: Tensions and Trade-offs of Land Registration and Titling in Sub-Saharan Africa. The Journal of Development Studies, 55(3), 384-400.

Borras, Saturnino M./Franco, Jennifer C. (2012): Global Land Grabbing and Trajectories of Agrarian Change: A Preliminary Analysis. Journal of Agrarian Change, 12(1), 34-59.

Borras, Saturnino M./Franco, Jennifer C. (2013): Global Land Grabbing and Political Reactions >From Below<. Third World Quarterly, 34(9), 1723-1747.

Borras, Saturnino M./Franco, Jennifer C./Gómez, Sergio/Kay, Cristóbal/Spoor, Max (2012): Land grabbing in Latin America and the Caribbean. The Journal of Peasant Studies, 39(3-4), 845-872.

Borras, Saturnino M./Franco, Jennifer C./Isakson, S. Ryan/Levidow, Les/Vervest, Pietje (2016): The rise of flex crops and commodities: implications for research. The Journal of Peasant Studies, 43(1), 93-115. 
Borras, Saturnino M./Kay, Cristóbal/Gomez, Sergio/Wilkinson, John (2012): Land grabbing and global capitalist accumulation: key features in Latin America. Canadian Journal of Development Studies, 33(4), 402-416.

Bourdieu, Pierre (1986): The Forms of Capital. In: Richardson, John G. (Hg.), Handbook of Theory and Research for the Sociology of Education. New York: Greenwood Press, 241-258.

Bradshaw, Alan (1976): A Critique of Steven Lukes' >Power: A Radical View<. Sociology, 10(1), 121-127.

Bräuchler, Birgit/Widlok, Thomas (2007): Die Revitalisierung von Tradition: Im (Ver-)Handlungsfeld zwischen staatlichem und lokalem Recht. Zeitschrift für Ethnologie, 132(1), 5-14.

Brautigam, Deborah (1992): Governance, Economy, and Foreign-Aid. Studies in Comparative International Development, 27(3), 3-25.

Bruce, Joy (2008): The Effect of the Biofuel Plantation on Communities in Northern Ghana. A Case Study Of 4 Communities. BioFuel Africa Ltd.

Brukum, Nana James Kwaku (2005): Traditional constitutions and Chieftaincy succession disputes in Northern Ghana. Legon Journal of Sociology, 2(1), 2232.

Brunner, Jan (2019): Land Grabbing in Ostdeutschland: Ursachen, Auswirkungen, Widerstand. Country Report Nr. 3. Berlin: GLOCON

Bryant, Raymond L./Bailey, Sinéad (1997): Third world political ecology. London, New York: Routledge.

Buckley-Zistel, Susanne (2005): The Truth Heals? Gacaca Jurisdictions and the Consolidation of Peace in Rwanda. Die Friedens-Warte, 80(1/2), 113-129.

Bull, Christian (2010): Norwegian Land Grabbers in Ghana - The case of ScanFuel. Oslo: Spire.

Burnod, Perrine/Gingembre, Mathilde/Ratsialonana, Rivo Andrianirina (2013): Competition over Authority and Access: International Land Deals in Madagascar. Development and Change, 44(2), 357-379.

Busscher, Nienke/Vanclay, Frank/Parra, Constanza (2019): Reflections on How State-Civil Society Collaborations Play out in the Context of Land Grabbing in Argentina. Land, 8(8), 1-16.

Buur, Lars/Kyed, Helene Maria (2007): State Recognition and Democratization in Sub-Saharan Africa : A new Dawn for Traditional Authorities? New York: Palgrave Macmillan.

Campion, Benjamin B./Acheampong, Emmanuel (2014): The Chieftaincy Institution in Ghana: Causers and Arbitrators of Conflicts in Industrial Jatropha Investments. Sustainability, 6(9), 6332-6350. 
Capps, Gavin (2016): Tribal-Landed Property: The Value of the Chieftaincy in Contemporary Africa. Journal of Agrarian Change, 16(3), 452-477.

Capps, Gavin (2018): Custom and exploitation: rethinking the origins of the modern African chieftaincy in the political economy of colonialism. The Journal of Peasant Studies, 45(5-6), 969-993.

Capps, Gavin/Mnwana, Sonwabile (2015): Claims from below: platinum and the politics of land in the Bakgatla-ba-Kgafela traditional authority area. Review of African Political Economy, 42(146), 606-624.

Chanock, Martin (1985): Law, custom, and social order : the colonial experience in Malawi and Zambia. Cambridge: Cambridge University Press.

Chanock, Martin (1991): A Peculiar Sharpness: An Essay on Property in the History of Customary Law in Colonial Africa. The Journal of African History, 32(01), 65-88.

Chimhowu, Admos/Woodhouse, Phil (2006): Customary vs Private Property Rights? Dynamics and Trajectories of Vernacular Land Markets in SubSaharan Africa. Journal of Agrarian Change, 6(3), 346-371.

Claassens, Aninka/Cousins, Ben (2008): Land, Power and Custom. Controversies generated by South Africa's Communal Land Rights Act. Athens: Ohio University Press.

Cotula, Lorenzo (2012): The international political economy of the global land rush: A critical appraisal of trends, scale, geography and drivers. Journal of Peasant Studies, 39(3-4), 649-680.

Cotula, Lorenzo/Berger, Thierry (Hg.) (2017): Improving accountability in agricultural investments: Reflections from legal empowerment initiatives in West Africa. London: IIED.

Cotula, Lorenzo/Oya, Carlos/Codjoe, Emmanuel A./Eid, Abdurehman/ Kakraba-Ampeh, Mark/Keeley, James/Kidewa, Admasu L./Makwarimba, Melissa/Seide, Wondwosen M./Nasha, William O./Asare, Richard O./Rizzo, Matteo (2014): Testing Claims about Large Land Deals in Africa: Findings from a Multi-Country Study. Journal of Development Studies, 50(7), 903-925.

Cotula, Lorenzo/Vermeulen, Sonja (2009): Deal or no deal: the outlook for agricultural land investment in Africa. International Affairs, 85(6), 1233-1247.

Crawford, Gordon/Botchwey, Gabriel (2017): Conflict, collusion and corruption in small-scale gold mining: Chinese miners and the state in Ghana. Commonwealth \& Comparative Politics, 1-28.

Crook, Richard C. (1986): Decolonization, the Colonial State, and Chieftaincy in the Gold Coast. African Affairs, 85(338), 75-105. 
Crowder, Michael (1964): Indirect Rule: French and British Style. Africa: Journal of the International African Institute, 34(3), 197-205.

Dahl, Robert A. (1957): The Concept of Power. Behavioral Science, 2(3), 201-215.

Dahl, Robert A. (1961): Who governs? Democracy and power in an American city. New Haven: Yale University Press.

Daley, Elizabeth (2011): Gendered Impacts of Commercial Pressures on Land. Commercial Pressures on Land: Mokoro, Cirad, International Land Coalition.

De Juan, Alexander (2017): »Traditional« Resolution of Conflicts: The Survival of Precolonial Dispute Settlement in Burundi. Comparative Political Studies, 50(13), 1835-1868.

De Schutter, Olivier (2011): How not to think of land-grabbing: three critiques of large-scale investments in farmland. The Journal of Peasant Studies, 38(2), 249-279.

de Soto, Hernan (2000): The Mystery of Capital: Why Capitalism Triumphs in the West and Fails Everywhere Else. New York: Basic Books.

de Sousa Santos, Boaventura (2016): Epistemologies of the South and the Future. From the European South, 1, 17-29.

Dell'Angelo, Jampel/D'Odorico, Paolo/Rulli, Maria Cristina/Marchand, Philippe (2017): The Tragedy of the Grabbed Commons: Coercion and Dispossession in the Global Land Rush. World Development, 92, 1-12.

Dietz, Kristina (2019): Direct democracy in mining conflicts in Latin America: mobilising against the La Colosa project in Colombia. Canadian Journal of Development Studies, 40(2), 145-162.

Dietz, Kristina/Engels, Bettina (2014): Immer (mehr) Ärger wegen der Natur - Für eine gesellschafts- und konflikttheoretische Analyse von Konflikten um Natur. Österreichische Zeitschrift für Politikwissenschaft, 43(1), 73-90.

Dietz, Kristina/Engels, Bettina (2017): Contested Extractivism, Society and the State: An Introduction. In: Engels, Bettina \& Dietz, Kristina (Hg.), Contested Extractivism, Society and the State. Struggles over Mining and Land. London: Palgrave Macmillan, 1-19.

Dietz, Kristina/Engels, Bettina (2018): Field of Conflict: Ein relationaler Ansatz zur Analyse von Konflikten um Land. GLOCON Working Paper Nr. 1. Berlin.

Dietz, Kristina/Engels, Bettina (2020): Analysing land conflicts in times of global crises. Geoforum, 111, 208-217.

Dogbevi, Emmanuel K. (23.02.2010): Scanfuel's Ghana Jatropha plantation wipes out settlements, farms. Ghana Business News. Abrufbar unter: https://www.ghanabusinessnews.com/2010/02/23/scanfuels-g 
hana-jatropha-plantation-wipes-out-settlements-farms/. Letzter Zugriff: 10.04 .

Dogbevi, Emmanuel K. (30.08.2010): Land grabbing for biofuels hits Ghana, other African countries - Report. Ghana Business News. Abrufbar unter: https:/www.ghanabusinessnews.com/2010/08/30/land-grabbingfor-biofuels-hits-ghana-other-african-countries-report/. Letzter Zugriff: 02.07.

Doss, Cheryl/Summerfield, Gale/Tsikata, Dzodzi (2014): Land, Gender, and Food Security. Feminist Economics, 20(1), 1-23.

Dowding, Keith (2006): Three-Dimensional Power: A Discussion of Steven Lukes' Power: A Radical View. Political Studies Review, 4(2), 136-145.

Drechsel, Franza/Engels, Bettina/Schäfer, Mirka (2019): »The mines make us poor«: Large-scale mining in Burkina Faso. Country Report Nr. 2. Berlin: GLOCON.

Dudenredaktion (o.J.): Matrilineal. Abrufbar unter: https://www.duden.de/re chtschreibung/matrilineal. Letzter Zugriff: 25.09.

Eckert, Andreas (1999): Grundbesitz, Landkonflikte und kolonialer Wandel: Douala 1880-1960. Stuttgart: Franz Steiner.

Eckert, Andreas (2007): Herrschen und Verwalten: afrikanische Bürokraten, staatliche Ordnung und Politik in Tanzania, 1920 - 1970. München: Oldenbourg.

Edelman, Marc/Oya, Carlos/Borras, Saturnino M. (2013): Global Land Grabs: historical processes, theoretical and methodological implications and current trajectories. Third World Quarterly, 34(9), 1517-1531.

Elhadary, Yasin Abdalla Eltayeb/Obeng-Odoom, Franklin (2012): Conventions, Changes, and Contradictions in Land Governance in Africa: The Story of Land Grabbing in North Sudan and Ghana. Africa Today, 59(2), 59-78.

Engels, Bettina (2019): Not normal, not just: protest against large-scale mining from a moral economy perspective. Canadian Journal of African Studies, 1-18.

Engels, Bettina (2021a): All good things come from below? Scalar constructions of the >locak in conflicts over mining. Political Geography, 84, 1-9.

Engels, Bettina (2021b): All good things come from below? Scalar constructions of the >locak in conflicts over mining. Political Geography, 84, 102295.

Engels, Bettina/Dietz, Kristina (2011): Land Grabbing analysieren: Ansatzpunkte für eine politisch-ökologische Perspektive am Beispiel Äthiopiens. Peripherie, 31(124), 399-420.

Engels, Bettina/Dietz, Kristina (Hg.) (2017): Contested Extractivism, Society and the State. Struggles over Mining and Land. London: Palgrave Macmillan. 
Englebert, Pierre (2002a): Born-again Buganda or the limits of traditional resurgence in Africa. Journal of Modern African Studies, 40(3), 345-368.

Englebert, Pierre (2002b): Patterns and theories of traditional resurgence in tropical Africa. Mondes en Developpement, 30(118), 51-64.

Eriksen, Stein Sundstøl (2001): The State We're In: Recent Contributions to the Debate on State-Society Relations in Africa. Forum for Development Studies, 28(2), 289-307.

Escobar, Arturo (1996): Construction nature: Elements for a post-structuralist political ecology. Futures, 28(4), 325-343.

Escobar, Arturo (2006): Difference and Conflict in the Struggle Over Natural Resources: A political ecology framework. Development, 49(3), 6-13.

Feyissa, Dereje (2005): Land and the Politics of Identity: The case of AnywaaNuer Relations in the Gambella Region. In: Evers, Sandra, Spierenburg, Marja, \& Wels, Harry (Hg.), Competing Jurisdictions : Settling land claims in Africa. Leiden, Boston: Brill.

FIAN (2010): Landraub in Ghana. Der Agrartreibstoffboom schmiert das Geschäft. Fact Sheet 2010 Nr. 3. Köln: FIAN.

Flick, Uwe (2014): Gütekriterien qualitativer Sozialforschung. In: Baur, Nina \& Blasius, Jörg (Hg.), Handbuch Methoden der empirischen Sozialforschung. Wiesbaden: Springer VS, 411-424.

Flyvbjerg, Bent (2011): Case Study. In: Denzin, Norman K. \& Lincoln, Yvonna S. (Hg.), The Sage handbook of qualitative research. Thousand Oaks: Sage, 301316.

FoodSPAN (2011): Report of Follow-up Activities on Land Grabs, Biofuel and Food Security, Ashanti Region. Accra: FoodSPAN, Action Aid international Ghana.

Franco, Jennifer C./Borras, Saturnino M. Jr. (Hg.) (2013): Land concentration, land grabbing and people's struggles in Europe. Amsterdam: Transnational Institute.

Gaventa, John (2006): Finding the Spaces for Change: A Power Analysis. IDS Bulletin, 37(6), 23-33.

Gerring, John (2007): Case Study Research. Principles and Practices. New York: Cambridge University Press.

Ghana Statistial Service (2014): 2010 Population and Housing Service. District Analytical Report. Mion District. Accra: Ghana Statistical Service.

Gilfoy, Kieran (2014): Land Grabbing and NGO Advocacy in Liberia: A Deconstruction of the »Homogenous Community«. African Affairs. 
glokal (2013): Mit kolonialen Grüßen... Berichte und Erzählungen von Auslandsaufenthalten rassismuskritisch betrachtet. Berlin: hinkelsteindruck.

GRAIN (2008): Seized! The 2008 land grab for food an financial security. GRAIN Briefing. Barcelona: GRAIN.

Graphic Online (28.08.2018): CONFIRMED: Results of the 2018 Referendum on new Regions. Graphic online. Abrufbar unter: https://www.graphic.co $\mathrm{m} . \mathrm{gh} / \mathrm{news} /$ politics/confirmed-results-of-the-2018-referendum-on-new -regions.html. Letzter Zugriff: 25.09. 2019.

Greco, Elisa (2015): Landlords in the making: class dynamics of the land grab in Mbarali, Tanzania. Review of African Political Economy, 42(144), 225-244.

Grischow, Jeff D. (2008): Rural >Community<, Chiefs and Social Capital: The case of Southern Ghana. Journal of Agrarian Change, 8(1), 64-93.

Hall, Ruth/Edelman, Marc/Borras, Saturnino M./Scoones, Ian/White, Ben/Wolford, Wendy (2015): Resistance, acquiescence or incorporation? An introduction to land grabbing and political reactions >from below The Journal of Peasant Studies, 42(3-4), 467-488.

Helfferich, Cornelia (2011): Die Qualität qualitativer Daten: Manual für die Durchführung qualitativer Interviews. Wiesbaden: VS.

Helfferich, Cornelia (2014): Das Leitfadeninterview. In: Baur, Nina \& Blasius, Jörg (Hg.), Handbuch Methoden der empirischen Sozialforschung. Wiesbaden: Springer VS, 559-574.

Helmke, Gretchen/Levitsky, Steven (2004): Informal Institutions and Comparative Politics: A Research Agenda. Perspectives on Politics, 2(04), 725-740.

Honig, Lauren (2017): Land, State-Building, and Political Autority in Africa. Dissertation, Cornell University, Ithaca.

Hunsberger, Carol (2010): The politics of Jatropha-based biofuels in Kenya: convergence and divergence among NGOs, donors, government officials and farmers. The Journal of Peasant Studies, 37(4), 939-962.

Hydén, Göran/Bratton, Michael (1992): Governance and politics in Africa. Boulder, Colorado: Lynne Rienner.

Iddrisu, Abdulai (2013): Dagomba. In: Danver, Steven L. (Hg.), Native peoples of the world. An Encyclopedia of Groups, Cultures, and Contemporary Issues. Oxon, New York: Routledge, 25-26.

Juul, Kristine/Lund, Christian (2002a): Negotiating property in Africa. Portsmouth: Heinemann.

Juul, Kristine/Lund, Christian (2002b): Negotiating property in Africa: Introduction. In: Juul, Kristine \& Lund, Christian (Hg.), Negotiating property in Africa. Portsmouth: Heinemann, 1-11. 
Kasanga, Kasim/Kotey, Nii Ashie (2001): Land Management in Ghana: Building on Tradition and Modernity. London: International Institute for Environment and Development.

Kirst, Sarah (2019): Traditionelle Autoritäten. In: Brunner, Jan, Dobelmann, Anna, Kirst, Sarah, \& Prause, Louisa (Hg.), Worterbuch Land- und Rohstoffkonflikte. Bielefeld: Transcript, 296-302.

Kirst, Sarah/Engels, Bettina (2012): Knappe Ressourcen und ihre soziale Vermittlung: Konflikte um Land im Südwesten Burkina Fasos. Research Report Peace and Conflict Studies Nr. 2. Berlin: Freie Universität Berlin, Research Unit Peace and Conflict Studies.

Kirst, Sarah/Engels, Bettina (2014): Land als knappe Ressource: Landkonflikte im Südwesten Burkina Fasos. In: Englert, Birgit \& Gärber, Barbara (Hg.), Land grabbing. Landnahmen in historischer und globaler Perspektive. Wien: New Academic Press, 117-130.

Kirst, Sarah/Prause, Louisa (2019): Lokale Gemeinschaft. In: Brunner, Jan, Dobelmann, Anna, Kirst, Sarah, \& Prause, Louisa (Hg.), Wörterbuch Land- und Rohstoffkonflikte. Bielefeld: Transcript, 239-244.

Kishindo, Paul (2004): Customary land tenure and the new land policy in Malawi. Journal of Contemporary African Studies, 22(2), 213-225.

Kleist, Nauja (2011): Modern chiefs: Tradition, development and return among traditional authorities in Ghana. African Affairs, 110(441), 629-647.

Knaup, Horand (05.09.2008): Green Gold Rush. Africa Becoming a Biofuel Battleground. Abrufbar unter: https://www.spiegel.de/international/wor ld/green-gold-rush-africa-becoming-a-biofuel-battleground-a-576548.ht ml. Letzter Zugriff: 13.11.

Knierzinger, Johannes (2011): Chieftaincy and Development in Ghana: From Political Intermediaries to Neotraditional Development Brokers. Arbeitspapiere Nr. 124: Institut für Ethnologie und Afrikastudien, Johannes GutenbergUniversität Mainz.

Kolnes, Steinar (2009): BioFuel Africa. Präsentation auf der Konferenz » Pressures on West African Land. Reconciling Development and Investment Policies«, 08.-10.12.2009, Bamako.

Kuba, Richard/Lentz, Carola (2003): Introduction: Histoire du Peuplement et Relations Interethniques au Burkina Faso. In: Kuba, Richard, Lentz, Carola, \& Somda, Claude Nurukyor (Hg.), Peuplement, Relations Interethniques et Identités. Paris: Karthala, 5-20. 
Kuusaana, Elias Danyi (2016): Large-scale land acquisitions for agricultural investments in Ghana - implications for land markets and smallholder farmers. Dissertation, Rheinische Friedrich-Wilhelms-Universität Bonn, Bonn.

Kuusaana, Elias Danyi/Bukari, Kaderi Noagah (2015): Land conflicts between smallholders and Fulani pastoralists in Ghana: Evidence from the Asante Akim North District (AAND). Journal of Rural Studies, 42, 52-62.

Kwoyiga, Lydia (2012): Institutional Analysis of Biofuel Production in Northern Ghana: Biofuel Africa Limited as a Case Study Norwegian University of Life Sciences, Oslo.

Kyed, Helene Maria/Buur, Lars (2007): Introduction: Traditional Authority and Democratization in Africa. In: Buur, Lars \& Kyed, Helene Maria (Hg.), State Recognition and Democratization in Sub-Saharan Africa : A New Dawn for Traditional Authorities? . New York: Palgrave Macmillan, 1-28.

Ladouceur, Paul (1972): The Yendi Chieftaincy Dispute and Ghanaian Politics. Canadian Journal of African Studies, 6(1), 97-115.

Lahiff, Edward/Borras, Saturnino M./Kay, Cristóbal (2007): Market-led agrarian reform: policies, performance and prospects. Third World Quarterly, 28(8), 1417-1436.

Lanz, Kristina/Gerber, Jean-David/Haller, Tobias (2018): Land Grabbing, the State and Chiefs: The Politics of Extending Commercial Agriculture in Ghana. Development and Change, 49(6), 1526-1552.

Larder, Nicolette (2015): Space for pluralism? Examining the Malibya land grab. The Journal of Peasant Studies, 42(3-4), 839-858.

Lavers, Tom (2012): Patterns of agrarian transformation in Ethiopia: Statemediated commercialisation and the sland grab<. Journal of Peasant Studies, 39(3-4), 795-822.

Lavigne Delville, Philippe (2000): Harmonising Formal Law and Customary Land Rights in French-Speaking West Africa. In: Toulmin, Camilla \& Quan, Julian (Hg.), Evolving land rights, policy and tenure in Africa. London: IIED, NRI, DFID, 97-122.

Le Billon, Philippe (2015): Environmental conflict. In: Perreault, Tom, McCarthy, James, \& Bridge, Gavin (Hg.), The Routledge Handbook of Political Ecology. London: Routledge, 598-608.

Lentz, Carola (2010): Is Land Inalienable? Historical and Current Debates on Land Transfers in Northern Ghana. Africa, 80(1), 56-80.

Li, Tania M. (2011): Centering labor in the land grab debate. Journal of Peasant Studies, 38(2), 281-298. 
Logan, Carolyn (2013): The roots of resilience: Exploring popular support for African traditional authorities. African Affairs, 112(448), 353-376.

Lukes, Steven (1974): Power: A Radical View. London, New York: Macmillan.

Lukes, Steven (2005): Power: A Radical View. Houndmills, Basingstoke, Hampshire, New York: Palgrave Macmillan.

Lund, Christian (1998): Struggles for Land and Political Power. The Journal of Legal Pluralism and Unofficial Law, 30(40), 1-22.

Lund, Christian (2006): Twilight institutions: Public authority and local politics in Africa. Development and Change, 37(4), 685-705.

Lund, Christian (2008): Local politics and the dynamics of property in Africa. New York: Cambridge University Press.

Lund, Christian (2011): Property and Citizenship: Conceptually Connecting Land Rights and Belonging in Africa. Africa Spectrum, 46(3), 71-75.

Lund, Christian (2013): The Past and Space: On Arguments in Africain Land Control. Africa, 83(Special Issue 01), 14-35.

Lund, Christian (2014): Of What is This a Case?: Analytical Movements in Qualitative Social Science Research. Human Organization, 73(3), 224-234.

Lund, Christian/Boone, Catherine (2013): Introduction: Land Politics in Africa - Constituting Authority over Territory, Property and Persons. Africa, 83(1), 1-13.

MacGaffey, Wyatt (2006): Death of a king, death of a kingdom? Social pluralism and succession to high office in Dagbon, northern Ghana. The Journal of Modern African Studies, 44(1), 79-99.

Mamdani, M. (2001): Beyond settler and native as political identities: Overcoming the political legacy of colonialism. Comparative Studies in Society and History, 43(4), 651-664.

Mamdani, Mahmood (1996): Citizen and subject : contemporary Africa and the legacy of late colonialism. Princeton, N.J.: Princeton University Press.

Martinez-Alier, Joan (2002): The Environmentalism of the Poor: A Study of Ecological Conflicts and Valuation. London: Elgar

Martinez-Alier, Joan/Temper/Del Bene, Daniela/Scheidel, Arnim (2016): Is there a global environmental justice movement? The Journal of Peasant Studies, 43(3), 731-755.

Mayring, Philipp (2002): Einführung in die qualitative Sozialforschung: Eine Anleitung zu qualitativem Denken (5. überarbeitete und neu ausgestattete Auflage ed.). Weinheim, Basel: Beltz Verlag. 
Mayring, Philipp (2014): Qualitative content analysis: theoretical foundation, basic procedures and software solution. Klagenfurt: gesis Leibniz-Institut für Sozialwissenschaften.

Mayring, Philipp/Brunner, Eva (2006): Qualitative Textanalyse - Qualitative Inhaltsanalyse. In: Flaker, Vito \& Schmid, Tom (Hg.), Von der Idee zur Forschungsarbeit. Wien: Böhlau, 453-462.

Mayring, Philipp/Fenzl, Thomas (2014): Qualitative Inhaltsanalyse. In: Baur, Nina \& Blasius, Jörg (Hg.), Handbuch Methoden der empirischen Sozialforschung. Wiesbaden: Springer 543-556.

Ministry of Lands and Forestry (1999): National Land Policy.

Mnwana, Sonwabile (2019): Chiefs, land and distributive struggles on the platinum belt, South Africa. In: Buthelezi, Mbonginseni, Skosana, Dineo, \& Vale, Beth (Hg.), Traditional Leaders in a Democracy: Resources, Respect and Resistance. Johannesburg: Jacana Press, 128-152.

Moe, Louise W./Simojoki, Maria Vargas (2013): Custom, contestation and cooperation: peace and justice promotion in Somaliland. Conflict, Security $\&$ Development, 13(4), 393-416.

Moore, Donald S. (2005): Suffering for territory: race, place, and power in Zimbabwe. Durham: Duke University Press.

Moore, Sally Falk (1978): Law as Process: An Anthropological Approach. London, Boston: Routledge, Kegan Paul.

Moore, Sally Falk (1986): Social Facts and Fabrications. "Customary« Law on Kilimanjaro, 1880-1980. Cambridge Cambridgeshire, New York: Cambridge University Press.

Moore, Sally Falk (1998): Changing African Land Tenure: Reflections on the Incapacities of the State. The European Journal of Development Research, 10(2), 33-49.

Moore, Sally Falk (1991): From giving and lending to selling: property transactions reflecting historical changes on Kilimanjaro. In: Mann, Kristin \& Roberts, Richard (Hg.), Law in colonial Africa. Portsmouth: Heinemann, 108-127.

Murphy, Susan/Carmody, Padraig/Okawakol, Julius (2017): When rights collide: land grabbing, force and injustice in Uganda. The Journal of Peasant Studies, 44(3), 677-696.

Mutisi, Marta/Sansculotte-Greenidge, Kwesi (2012): Integrating Traditional and Modern Conflict Resolution: Experiences from Selected Cases in Eastern and the Horn of Africa: African Centre for the Constructive Resolution of Disputes (ACCORD). 
Ndi, Frankline/Batterbury, Simon (2017): Land Grabbing and the Axis of Political Conflicts: Insights from Southwest Cameroon. Afrika Spectrum, 52(1), 33.

Nkrumah, Kwame (1961): I speak of freedom: A statement of African ideology. London: Heinemann.

Nolte, Kerstin (2013): Large-Scale Agricultural Investments under Poor Land Governance Systems: Actors and Institutions in the Case of Zambia. GIGA Working Papers Nr. 221. Hamburg: German Institute of Global and Area Studies (GIGA).

Nolte, Kerstin/Chamberlain, Wytske/Giger, Markus (2016): International Land Deals for Agriculture. Fresh insights from the Land Matrix: Analytical Report II. Bern: Bern Open Publishing.

Nolte, Kerstin/Väth, Susanne Johanna (2015): Interplay of land governance and large-scale agricultural investment: evidence from Ghana and Kenya. The Journal of Modern African Studies, 53(01), 69-92.

Nyari, Bakari (2008): Biofuel Land Grabbing in Northern Ghana. Abrufbar unter: https://www.biofuelwatch.org.uk/wp-content/uploads/biofuels_g hana.pdf. Letzter Zugriff: 03.07.

Nygaard, Ivan/Bolwig, Simon (2018): The rise and fall of foreign private investment in the jatropha biofuel value chain in Ghana. Environmental Science $\mathcal{E}$ Policy, 84, 224-234.

Obeng-Odoom, Franklin (2016): Understanding Land Reform in Ghana: A Critical Postcolonial Institutional Approach. Review of Radical Political Economics, 48(4), 661-680.

OECD (2001): The DAC Guidelines: Helping Prevent Violent Conflict. Paris: OECD.

OECD/DAC (1996): "Shaping the 21st Century: The Contribution of Development Co-operation«. Paris: Organization for Economic Co-operation and Development, Development Assistance Committee.

Offe, Claus (1977): Einleitung. In: Bachrach, Peter \& Baratz, Morton S. (Hg.), Macht und Armut. Eine theoretisch-empirische Untersuchung. Frankfurt a.M.: Suhrkamp, 7-34.

Olivier de Sardan, Jean-Pierre (2011): The Eight Modes of Local Governance in West Africa. IDS Bulletin, 42(2), 22-31.

Oya, Carlos (2013): The Land Rush and Classic Agrarian Questions of Capital and Labour: a systematic scoping review of the socioeconomic impact of land grabs in Africa. Third World Quarterly, 34(9), 1532-1557.

Peluso, Nancy Lee/Lund, Christian (2011): New frontiers of land control: Introduction. Journal of Peasant Studies, 38(4), 667-681. 
Peluso, Nancy Lee/Ribot, Jesse (2020): Postscript: A Theory of Access Revisited. Society \& Natural Resources, 33(2), 300-306.

Peluso, Nancy Lee/Watts, Michael (2001): Violent environments. Ithaca: Cornell University Press.

Peters, Pauline E. (1984): Struggles over Water, Struggles over Meaning - Cattle, Water and the State in Botswana. Africa, 54(3), 29-49.

Peters, Pauline E. (1994): Dividing the Commons : Politics, Policy, and Culture in Botswana. Charlottesville: University Press of Virginia.

Peters, Pauline E. (2004): Inequality and Social Conflict Over Land in Africa. Journal of Agrarian Change, 4(3), 269-314.

Peters, Pauline E. (2010): »Our daughters inherit our land, but our sons use their wives' fields «: matrilineal-matrilocal land tenure and the New Land Policy in Malawi. Journal of Eastern African Studies, 4(1), 179-199.

Peters, Pauline E. (2013a): Conflicts over land and threats to customary tenure in Africa. African Affairs, 112(449), 1-20.

Peters, Pauline E. (2013b): Land appropriation, surplus people and a battle over visions of agrarian futures in Africa. The Journal of Peasant Studies, 40(3), 537-562.

Pfaff-Czarnecka, Joanna (2011): From »identity« to »belonging« in social research: plurality, social boundaries, and the politics of the self. Working Papers in Development Sociology and Social Anthropology Nr. Bielefeld: Universität Bielefeld, Fakultät für Soziologie, AG Sozialanthropologie.

Polsby, Nelson W. (1960): How to Study Community Power: The Pluralist Alternative. The Journal of Politics, 22(3), 474-484.

Porro, Noemie Miyasaka (2010): Conclusion. For a Politics of Difference. In: Tsikata, Dzodzi \& Golah, Pamela (Hg.), Land Tenure, Gender and Globalization: Research and Analysis from Africa, Asia and Latin America. Ottawa: International Development Research.

Prause, Louisa (2019): Success and failure of protest actors' framing strategies in conflicts over land and mining in Senegal. Canadian Journal of Development Studies, 40(3), 387-403.

Prause, Louisa/Le Billon, Philippe (2020): Struggles for land: comparing resistance movements against agro-industrial and mining investment projects. The Journal of Peasant Studies, 1-24.

Quaye, Benjamin Armah (2006): Towards an appropriate framework for the effective utilization/management of geoinformation: a case study of Ghana. Paper präsentiert auf derShaping the Change. XXIII FIG Conference, München. 
Rangan, Haripriya (1997): Property vs. Control: The State and Forest Management in the Indian Himalaya. Development and Change, 28(1), 71-94.

Ranger, Terence (1983): The invention of tradition in colonial Africa. In: Hobsbawm, Eric \& Ranger, Terence (Hg.), The Invention of Tradition. Cambridge: Cambridge University Press, 211-262.

Ray, Donald I./Reddy, P. S. (Hg.) (2003): Grassroots governance? Chiefs in Africa and the Afro-Caribbean. Calgary: University of Calgary Press.

Republic of Ghana (1962a): Administration of Lands Act, 123.

Republic of Ghana (1962b): State Lands Act, 125, Government Printing Department.

Republic of Ghana (1992): Constitution of the Republic of Ghana.

Ribot, Jesse C. (1998): Theorizing access: Forest profits along Senegal's charcoal commodity chain. Development and Change, 29(2), 307-341.

Ribot, Jesse C./Peluso, Nancy Lee (2003): A theory of access. Rural Sociology, 68(2), 153-181.

Robbins, Paul (2012): Political ecology : A Critical Introduction. Malden, Oxford, Chichester: Wiley-Blackwell

Robbins, Paul (2008): The State in Political Ecology: A Postcard to Political Geography from the Field. In: Cox, Kevin R., Low, Murray, \& Robinson, Jennifer (Hg.), The SAGE handbook of political geography. London: SAGE, 205219.

Roelf, Wendell (21.11.2008): Interview: Scanfuel to start Ghana biofuel Operation. Oil Report. Abrufbar unter: https://in.reuters.com/article/bio fuels-ghana/interview-scanfuel-to-start-ghana-biofuel-operation-idINL K55111220081121. Letzter Zugriff.

Rukuni, Tinashe/Machingambi, Zadzisai/Musingafi, Maxwell C.C./Kaseke, Kwaedza E. (2015): The Role of Traditional Leadership in Conflict Resolution and Peace Building in Zimbabwean Rural Communities: The Case of Bikita District. Public Policy and Administration Research, 5(3), 75-79.

SAL (2017): Proposed commercial farming project at Botanga in the Kumbungu District of the Northern Region. Environmental and Social Management Plan. SAL Consult Limited, Accra.

Santschi, Martina (2014): Traditional Authorities, Local Justice and Local Conflict Resolution Mechanisms in South Sudan. In: Hellmüller, Sara \& Santschi, Martina (Hg.), Is Local Beautiful? Cham: Springer International Publishing, 43-63.

Schattschneider, Elmer Eric (1960): The Semisovereign People. A Realist's View of Democracy in America. New York: Holt, Rinehart and Winston. 
Schenk, Mario (2018): Die Rolle des Staates in Konflikten um land grabbing in Nordbrasilien. GLOCON Working Paper Series Nr. 8. Berlin.

Schoneveld, George C./German, Laura A./Nutakor, Eric (2011): Land-based Investments for Rural Development? A Grounded Analysis of the Local Impacts of Biofuel Feedstock Plantations in Ghana. Ecology and Society, 16(4), Ohne Seitenzahlen.

Schoneveld, George Christoffel/German, Laura (2014): Translating Legal Rights into Tenure Security: Lessons from the New Commercial Pressures on Land in Ghana. The Journal of Development Studies, 50(2), 187-203.

Schrader, Svenja (2014): Landnahmen für Agrarkraftstoffe in Ghana. Das Beispiel des Jatropha Anbaus. In: Englert, Birgit \& Gärber, Barbara (Hg.), Land Grabbing. Landnahmen in historischer und globaler Perspektive. Wien: New Academic Press, 168-185.

Schuck, Hartwig (2014): Wie objektiv sind Interessen? Facetten und Funktionen des Interessenbegriffs in kritischen Analysen sozialer Verhältnisse. Zeitschrift für kritische Sozialtheorie und Philosophie, 41(1), 298-324.

Scoones, Ian/Hall, Ruth/Borras, Saturnino M./White, Ben/Wolford, Wendy (2013): The politics of evidence: methodologies for understanding the global land rush. Journal of Peasant Studies, 40(3), 469-483.

Seawright, Jason/Gerring, John (2008): Case Selection Techniques in Case Study Research: A Menu of Qualitative and Quantitative Options. Political Research Quarterly, 61(2), 294-308.

SGS Environment (2009): BioFuel Africa Limited. Environmental Assessment and Audit Report Jade Project. Accra: SGS Environment.

Shipton, Parker/Goheen, Mitzi (1992): Introduction. Understanding African Land-Holding: Power, Wealth, and Meaning. Africa: Journal of the International African Institute, 62(3), 307-325.

Sibeon, Roger (2004): Rethinking social theory. London, Thousand Oaks: Sage Publications.

Sikor, Thomas/Lund, Christian (2009a): Access and Property: A Question of Power and Authority. Development and Change, 40(1), 1-22.

Sikor, Thomas/Lund, Christian (2009b): The Politics of Possession: Property, Authority and Access to Natural Resources. Chichester, West Sussex, U.K. ; Malden, MA: Wiley-Blackwell.

Sjaastad, E./Cousins, B. (2009): Formalisation of land rights in the South: An overview. Land Use Policy, 26(1), 1-9.

Stacey, Paul (2015): Political Structure and the Limits of Recognition and Representation in Ghana. Development and Change, 46(1), 25-47. 
Stake, Robert E. (1995): The Art of Case Study Research Thousand Oaks, CA: SAGE. Stake, Robert E. (2003): Case studies. In: Denzin, Norman K. \& Lincoln, Yvonna S. (Hg.), Handbook of Qualitative Research. Thousand Oaks, CA: SAGE, 134-164.

Tarrow, Sidney (2010): The Strategy of Paired Comparison: Toward a Theory of Practice. Comparative Political Studies, 43(2), 230-259.

The Land Matrix (2019): Global: Ghana. Abrufbar unter: https://landmatrix.or g/data/by-target-country/ghana/. Letzter Zugriff: 27.06.2020.

The Land Matrix (2020): Web of Transnational Deals. Abrufbar unter: htt ps://landmatrix.org/charts/web-of-transnational-deals/. Letzter Zugriff: 17.06.2020.

The New Humanitarian (09.09. 2009): Land grabs force hundreds off farm, growers say. The New Humanitarian. Abrufbar unter: www.thenewhumanitarian.org/report/86044/ghana-land-grabs-force-hundredsfarms-growers-say. Letzter Zugriff: 21.11.2019.

The World Factbook (2016). Washington, DC: Central Intelligence Agency.

Tonah, Steve (2012): The Politicisation of a Chieftaincy Conflict: The case of Dagbon, Northern Ghana. Nordic Journal of African Studies, 21(1), 1-20.

Toulmin, Camilla (2009): Securing land and property rights in sub-Saharan Africa: The role of local institutions. Land Use Policy, 26(1), 10-19.

Toulmin, Camilla/Quan, Julian (2000): Evolving land rights, tenure and policy in sub-Saharan Africa. In: Toulmin, Camila \& Quan, Julian (Hg.), Evolving Land Rights, Policy and Tenure in Africa. London: Department for International Development/International Institute for Environment and Development/Natural Resources Institute, 207-228.

Tsikata, Dzodzi/Yaro, Joseph (2011): Land market liberalization and trans-national commercial land deals in Ghana since the 1990s. Paper präsentiert auf derInternational Conference of Global Land Grabbing, 6-8 April, University of Sussex.

Tubiana, Jérôme/Tanner, Victor/Abdul-Jalil, Musa Adam (2012): Traditional Authorities' Peacemaking Role in Darfur. Peaceworks Nr. 83: United States Institute of Peace.

Turner, J. H. (1975): Marx and Simmel Revisited - Reassessing Foundations of Conflict Theory. Social Forces, 53(4), 618-627.

Ubink, Janine M. (2008a): In the Land of the Chiefs. Customary Law, Land Conflicts, and the Role of the State in Peri-Urban Ghana. Leiden: Leiden University Press. 
Ubink, Janine M. (2008b): Negotiated or Negated? The Rhetoric and Reality of Customary Tenure in an Ashanti village in Ghana. Africa, 78(2), 264.

Ubink, Janine M. (2008c): Traditional authorities in Africa: Resurgence in an era of democratisation. Leiden: Leiden University Press.

United Nations General Assembly (1966): International Covenant on Economic, Social and Cultural Rights, United Nations.

van der Ploeg, Jan Douwe/Franco, Jennifer C./Borras, Saturnino M. (2015): Land concentration and land grabbing in Europe: a preliminary analysis. Canadian Journal of Development Studies/Revue canadienne d'études du développement, 36(2), 147-162.

van Rouveroy van Nieuwaal, Emile Adriaan B. (1996): States and Chiefs. Are chiefs mere puppets? The Journal of Legal Pluralism and Unofficial Law, 28 (3738), 39-78.

Vandergeest, Peter/Peluso, Nancy (1995): Territorialization and state power in Thailand. Theory and Society, 24(3), 385-426.

VeneKlasen, Lisa/Miller, Valerie (2002): A New Wave of Power, People \& Politics: The Action Guide for Advocacy and Citizen Participation. Oklahoma City: World Neighbors.

Vermeulen, Sonja/Cotula, Lorenzo (2010): Over the heads of local people: consultation, consent, and recompense in large-scale land deals for biofuels projects in Africa. Journal of Peasant Studies, 37(4), 899-916.

Vogl, Susanne (2014): Gruppendiskussion. In: Baur, Nina \& Blasius, Jörg (Hg.), Handbuch Methoden der empirischen Sozialforschung. Wiesbaden: Springer VS, 581-586.

von Benda-Beckmann, Franz (2002): Who is afraid of legal pluralism? Paper präsentiert auf derXIIIth Conference of the Commission on Folk Law and Legal Pluralism, Chianmei, Thailand.

von Maltitz, Graham/Gasparatos, Alexandros/Fabricius, Christo (2014): The Rise, Fall and Potential Resilience Benefits of Jatropha in Southern Africa. Sustainability, 6(6), 3615-3643.

von Trotha, Trutz (1996): From Administrative to Civil Chieftaincy. The Journal of Legal Pluralism and Unofficial Law, 28(37-38), 79-107.

von Trotha, Trutz (2000): Die Zukunft liegt in Afrika. Vom Zerfall des Staates, von der Vorherrschaft der konzentrischen Ordnung und vom Aufstieg der Parastaatlichkeit. Leviathan, 28(2), 253-279.

Watts, Michael/Peet, Richard (2004): Liberating Political Ecology. In: Watts, Michael; Peet, Richard (Hg.), Liberation Ecologies, Second Edition. Environment, development, social movements. London, New York: Routledge, 3-47. 
Weber, Max (1972 [1922]): Wirtschaft und Gesellschaft : Grundriss der verstehenden Soziologie. Tübingen: Mohr Siebeck.

White, Ben/Borras, Saturnino M./Hall, Ruth/Scoones, Ian/Wolford, Wendy (2012): The new enclosures: critical perspectives on corporate land deals. The Journal of Peasant Studies, 39(3-4), 619-647.

Whitehead, Ann/Tsikata, Dzodzi (2003): Policy Discourses on Women's Land Rights in Sub-Saharan Africa: The Implications of the Re-turn to the Customary. Journal of Agrarian Change, 3(1-2), 67-112.

Wilson, Bradley R. (2015): Reclaiming the worker's property: control grabbing, farmworkers and the Las Tunas Accords in Nicaragua. The Journal of Peasant Studies, 42(3-4), 747-763.

Wissen, Markus (2015): The political ecology of agrofuels: conceptual remarks. In: Dietz, Kristina, Engels, Bettina, Pye, Oliver, \& Brunnengräber, Achim (Hg.), The political ecology of agrofuels. London, New York: Routledge, 16-33.

Wolford, Wendy/Borras, Saturnino M./Hall, Ruth/Scoones, Ian/White, Ben (2013): Governing Global Land Deals: The Role of the State in the Rush for Land. Development and Change, 44(2), 189-210.

World Bank (1989): From crisis to sustainable growth: the long term perspective study on sub-Saharan Africa. Washington, D.C.: The World Bank.

World Bank (1992): Governance and Development. Washington, DC: The World Bank.

World Bank (1994): Adjustment in Africa: Reforms, results and the road ahead. Washington, DC: The World Bank.

World Bank (2002): Ghana - Promoting Partnerships with Traditional Authorities Project. Washington, DC: World Bank.

World Bank (2003): Project appraisal document on a proposed credit in the amount of SDR 15.1 million (US\$20.5 million equivalent) to the Republic of Ghana for a land administration project: The World Bank.

World Bank (2004): World Development Report 2005 : A Better Investment Climate for Everyone. New York: The World Bank, Oxford University Press.

World Bank (2013): Project Performance Assessment Report Ghana Land Administration Project. Washington D.C.: The World Bank.

Yaro, Joseph A. (2013): Neoliberal globalisation and evolving local traditional institutions: implications for access to resources in rural northern Ghana. Review of African Political Economy, 40(137), 410-427.

Yaro, Joseph A./Tsikata, Dzodzi (2014): Neo-traditionalism, chieftaincy and land grabs in Ghana. Policy Brief. Nr. 74. Brighton: Future Agricultures Consortium Secretariat at the University of Sussex. 
Yin, Robert K. (2009): Case Study Research: Design and Methods. Los Angeles: SAGE.

Yirrah, Nana Ama (2012): Land Grabbing, Biofuel Investments and Traditional Authorities in Ghana. Policy Brief: International Food Security Network/Action Aid Ghana/Food Security Policy Advocacy Network,.

Zartman, I. William (2000): Traditional cures for modern conflicts : African conflict »medicine«. Boulder: Lynne Rienner. 



\section{Glossar}

Community land Flächen, die Mitgliedern einer Gemeinschaft sowie Migrant*innen zur kollektiven oder individuellen Nutzung vorbehalten sind. Den Zugang zu diesen Flächen kontrolliert der sub-chief, der für die Verwaltung des Lands in der jeweiligen Gegend zuständig ist.

Customary Dauerhaftes, individuelles Recht auf Zugang zu Land, freehold

Drink money das neben der eigenen Nutzung ermöglicht, Land zu vererben und es anderen Landnutzer*innen zur Verfügung zu stellen. Über einen customary freehold verfügen in der Regel Landnutzer*innen einer bestimmten Abstammungslinie.

Eine kleinere Summe Geld, die traditionellen Autoritäten als Zeichen des Respekts erhalten wann immer ihnen jemand ein Anliegen unterbreitet. Früher war es üblich ihnen eine symbolische Gabe - typischerweise eine Flasche Schnapps oder Kola-Nüsse - zu überreichen. Seit einiger Zeit ist es gängige Praxis diese Gaben immer häufiger durch kleine Summen Geld zu ersetzen. Mit Bezug auf den früheren Brauch trägt dieses die Bezeichnung drink money.

Elders Traditionelle Autoritäten, die unter anderem als Berater*innen für in der Hierarchie höher stehende traditionelle Autoritäten fungieren.

Farming Siedlungen, deren Bewohner*innen Landnutzer*innen community sind, die nur in der Anbau- und Erntesaison dort leben und deren Hauptwohnsitz sich an einem anderen Ort meist in nahegelegenen Städten - befindet. 
Paramountchief Traditionelle Autorität von höchster Rangordnung in Ghana, die für die gewohnheitsrechtliche Verwaltung eines traditionell verwalteten Gebiets zuständig ist. Der paramount chief ist immer der chief der Hauptstadt dieser Gegend und der Vorsitzende des traditional council. Er hat rangniedrigere traditionelle Autoritäten wie subchiefs unter sich.

\section{Settler chief Chief einer settler community}

Settler community Eine Siedlung, in der sich Migrant*innen niedergelassen haben, denen eine traditionelle Autorität aus der Gegend Zugang zu dem durch sie verwaltete Land gewährt hat.

Sharecropping Eine Form der Pacht, die sich dadurch auszeichnet, dass die Pächter*innen (sharecropper) einen zuvor vereinbarten Anteil der erwirtschafteten Ernte als Pacht an diejenigen abgeben, die ihnen Zugang zu Land gewähren.

Skin

Die Gesamtheit einer traditionellen Gemeinschaft im Norden Ghanas. Dort sitzen traditionelle Autoritäten bei ihrer Ernennung zum chief sowie zu offiziellen Anlässen auf Tierhäuten (skins), die ihre Macht verkörpern.

Skin land Gewohnheitsrechtlich verwaltetes Land im Norden Ghanas. Skin land bezeichnet entweder das gesamte Land in einer traditional area oder Land, das einem chief für sich und seine Angehörigen vorbehalten ist.

Stool

Die Gesamtheit einer traditionellen Gemeinschaft im Süden Ghanas. Dort sitzen traditionelle Autoritäten bei ihrer Ernennung zum chief sowie zu offiziellen Anlässen auf hölzernen Schemeln (stools), die ihre Macht verkörpern.

Stool land Gewohnheitsrechtlich verwaltetes Land im Süden Ghanas. Stool land bezeichnet entweder das gesamte Land in einer traditional area oder Land, das einem chief für sich und seine Angehörigen vorbehalten ist.

Sub-chief Traditionelle Autorität von niedrigem Rang, die einem Dorf vorsteht.

Queenmother Weibliche traditionelle Autorität. Im chieftaincy-System der Ashanti ist sie für die Nominierung des paramount chiefs zuständig 
Traditional area Ein gewohnheitsrechtlich verwaltetes Gebiet, das aus verschiedenen kleineren Gemeinschaften besteht.

Traditional council Die Regierung einer traditional area. Der Vorstehende eines traditional council ist der paramount chief. Außerdem gehören dem traditional council die unter dem paramount chief stehenden sub-chiefs, die auch als elders bezeichnet werden, sowie die queenmother an.

Usufruct rights Traditionelle Landrechte, die den Zugang zu Land an die Nutzung des Lands knüpfen. Für Mitglieder einer indigenen Gemeinschaft sind diese Rechte permanent und können auch vererbt werden so lange das Land genutzt wird. Sie können jedoch nicht an Dritte weitergegeben werden. Für Migrant*innen ist der Erhalt dieser Rechte in der Regel mit einer geldlichen Gegenleistung oder Abgaben in Form von Ernteanteilen verbunden. 



\section{Abkürzungsverzeichnis}

ATC: Agogo Traditional Council

CICOL: Civil Society Coalition on Land

CLS: Customary Land Secretariat

EPA: Environmental Protection Agency

ESIA: Environmental and Social Impact Assessment

GCAP: Ghana Commercial Agriculture Project

GHS: Ghanaischer Cedi

LAP: Land Administration Project

NCO: Non-Governmental Organization

OASL: Office of the Administrator of Stool Lands 



\section{Abbildungsverzeichnis}

Abbildung 1: Wahrgenommene Zuständigkeitsverteilung zwischen Lokalregierung und traditionellen Autoritäten in der Konfliktlösung, S. 97.

Abbildung 2: Wahrgenommene Zuständigkeitsverteilung zwischen Lokalregierung und traditionellen Autoritäten in der Landvergabe, S. 98.

Abbildung 3: Ablaufmodell induktiver Kategorienbildung und deduktiver Kategorienanwendung, S. 113.

Abbildung 4: Darstellung des durch einen paramount chief verwalteten Lands, S. 126.

Abbildung 5: Traditionelle Landrechte in Ghana, S. 128.

Abbildung 6: Karte der Regionen Ghanas und den Projektstandorten von ScanFarm und BioFuel Africa, S. 136.

Abbildung 7: Karte der Projektgegend von ScanFarm, S. 137.

Abbildung 8: Karte der betroffenen Dörfer des BioFuel Africa-Projekts, S. 148. 



\section{Tabellenverzeichnis}

Tabelle 1: Übersicht über die drei Dimensionen der Macht, S. 78.

Tabelle 2: Operationalisierung der theoretischen Kategorien, S. 89. 


\section{Politikwissenschaft}

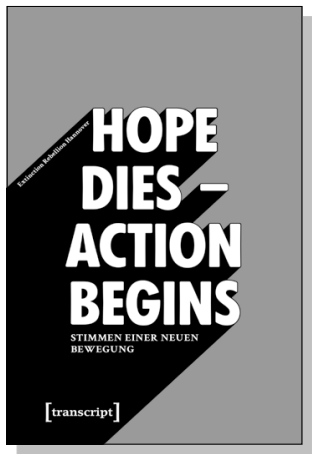

Extinction Rebellion Hannover

"Hope dies - Action begins":

Stimmen einer neuen Bewegung

2019,96 S., kart.

7,99€ (DE), 978-3-8376-5070-9

E-Book: kostenlos erhältlich als Open-Access-Publikation

PDF: ISBN 978-3-8394-5070-3

EPUB: ISBN 978-3-7328-5070-9

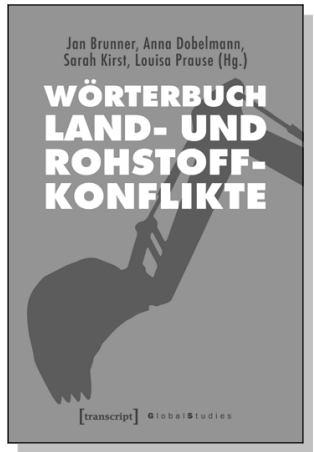

Jan Brunner, Anna Dobelmann, Sarah Kirst, Louisa Prause (Hg.)

Wörterbuch Land- und Rohstoffkonflikte

2019, 326 S., kart., Dispersionsbindung, 1 SW-Abbildung 24,99€ (DE), 978-3-8376-4433-3

E-Book:

PDF: $21,99 €$ (DE), ISBN 978-3-8394-4433-7

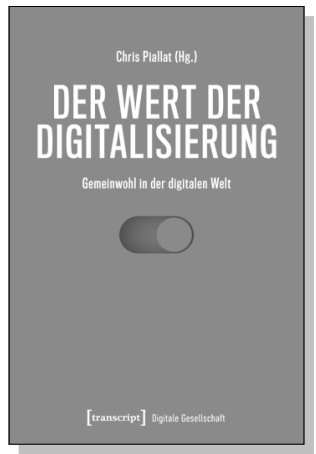

Chris Piallat (Hg.)

\section{Der Wert der Digitalisierung}

Gemeinwohl in der digitalen Welt

August 2021, 440 S., kart.,

Dispersionsbindung, 5 SW-Abbildungen, 3 Farbabbildungen 29,50€ (DE), 978-3-8376-5659-6

E-Book: kostenlos erhältlich als Open-Access-Publikation

PDF: ISBN 978-3-8394-5659-0

EPUB: ISBN 978-3-7328-5659-6 


\section{Politikwissenschaft}

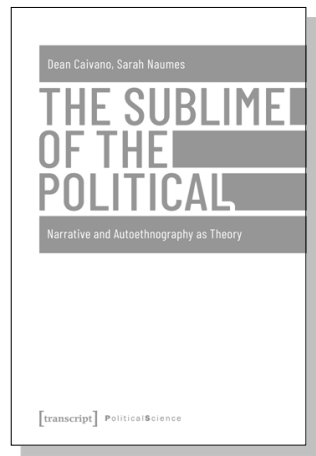

Dean Caivano, Sarah Naumes

The Sublime of the Political

Narrative and Autoethnography as Theory

July 2021, 162 p., hardcover

$100,00 €(D E), 978-3-8376-4772-3$

E-Book:

PDF: 99,99€ (DE), ISBN 978-3-8394-4772-7

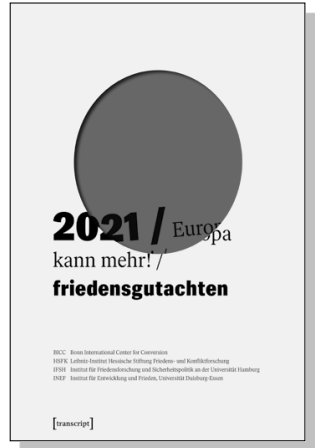

BICC Bonn International Center for Conversion, HSFK Leibniz-Institut Hessische Stiftung Friedens- und Konfliktforschung, IFSH Institut für Friedensforschung und Sicherheitspolitik an der Universität Hamburg, INEF Institut für Entwicklung und Frieden

\section{Friedensgutachten 2021}

Europa kann mehr!

Juni 2021, 160 S., kart.,

Dispersionsbindung, 22 Farbabbildungen

$15,00 €(D E), 978-3-8376-5786-9$

E-Book: kostenlos erhältlich als Open-Access-Publikation PDF: ISBN 978-3-8394-5786-3

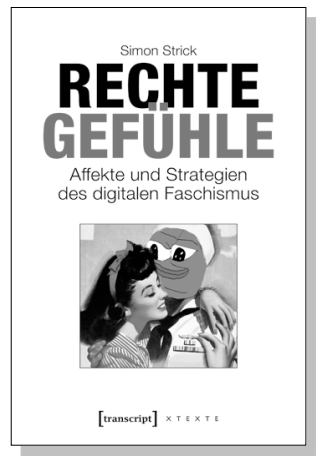

Simon Strick

\section{Rechte Gefühle}

Affekte und Strategien des digitalen Faschismus

Mai 2021, 480 S., kart.,

Dispersionsbindung, 170 SW-Abbildungen, 6 Farbabbildungen 34,00€ (DE), 978-3-8376-5495-0

E-Book:

PDF: 33,99 € (DE), ISBN 978-3-8394-5495-4 
